\title{
Characterization of Alpha Decays and Detector Response and Search for 5.5 MeV Solar Axions in DEAP-3600
}

by

\author{
Carl Rethmeier
}

\author{
A Dissertation submitted to \\ the Faculty of Graduate and Postdoctoral Affairs \\ in partial fulfilment of \\ the requirements for the degree of
}

Doctor of Philosophy

Ottawa-Carleton Institute for

Physics

Department of Physics
Carleton University
Ottawa, Ontario, Canada
September 16, 2021

Copyright (C)

2021 - Carl Rethmeier 


\section{Abstract}

DEAP-3600 is a liquid argon (LAr) based spin-independent direct dark matter search experiment. It is designed to detect nuclear recoils induced by the elastic scattering of weakly interacting massive particles (WIMPs) on argon nuclei. In 2019, DEAP-3600 reported the best upper limit on the WIMP-nucleon spin-independent cross section on a LAr target of $3.9 \times 10^{-45} \mathrm{~cm}^{2}$ for a $100 \mathrm{GeV} / \mathrm{c}^{2}$ WIMP mass at $90 \% \mathrm{CL}$. An essential component of this result involved measuring the rates of $\alpha$-decays within the detector and determining their impact on the expected background rate in the WIMP search region. The techniques used to measure and characterize these rates in-situ, and their results, will be discussed here in detail.

Like $\alpha$ decays, neutron scatters off LAr nuclei also produce nuclear-recoils, potentially mimicking a WIMP interaction. A good estimate of the expected number of neutron scatters in the WIMP region of interest is, therefore, essential for performing a search for WIMP interactions. This estimate was revised and improved for the upcoming DEAP-3600 WIMP search result, which will include approximately 800 live-days of data. The details and results of this analysis are included in this report.

In addition, DEAP-3600's large target mass and excellent ability to distinguish between electronic and nuclear recoils makes it well-suited for the detection of $5.5 \mathrm{MeV}$ solar axions, which would produce electronic recoils in the LAr, at higher energy than most backgrounds. The $5.5 \mathrm{MeV}$ solar axion search analysis, including the calibration of the energy response function on AmBe neutron calibration data, development of 
the Monte Carlo based background and signal models, the algorithm developed to fit the MC model to the data using Bayesian techniques, corrections made to the GEANT4 neutron capture spectrum, and the approach that will be used to calculate the final result will be discussed in detail. 


\section{Acknowledgments}

I would like to thank Kevin Graham for his guidance and mentorship during my journey through graduate school. He taught me how to communicate, work, and think like a scientist. He also made sure to prioritize the person before the project, always considering it of utmost importance that I enjoy the work that I do, and taking a strong interest in my personal life and well-being. He formed me into a scientist and I could not have asked for a better supervisor!

I would like to thank the DEAP Collaboration, particularly the people at Carleton, for their friendship and support of my work. Everyone I met was always willing to answer questions and lend a hand. I would like to thank Matt Dunford for getting me started with DEAP, and for sharing his advice and knowledge when I first joined, and Andrew Erlandson and David Gallacher for their help and advice as I ran into issues with the software or when I just needed good ideas. I want to thank Tina for helping me learn how to use the DEAP DB and for supporting me as DB co-admin, Simon Viel and Marcin Kuźniak for their support and advice, Björn Lehnert and Alexander Derbin for lending their expertise to the axion work, and Hamid Moradi for excellent discussions that slowed down my $\mathrm{PhD}$ work.

I would especially like to thank Rob Stainforth and Shawn Westerdale. They both put so much of their time and effort into helping me with the $\alpha$ and axion work that is described in this thesis. Without their time, extensive knowledge, advice, and constant support, I wouldn't have been able to do it. Both were always ready and 
available to answer questions, and frequently took the time to provide very detailed feedback on my work.

My parents worked very hard to provide me and my siblings with a quality education growing up, and always encouraged me to pursue a career I was passionate about. My parents and siblings, Natasha, Rebecca, and Joseph, made me the person I am today through their constant love and support throughout my entire life.

And lastly, I owe a deep debt of gratitude to my wife, Gloria. She put up with the often long hours of work I had to put in to accomplish this, and her constant love and support got me through it. My wife and children, Christopher and Veronica, have all been constant sources of joy and have been me going, especially during the most difficult times of my PhD work. 


\section{Table of Contents}

Abstract ii

Acknowledgments $\quad$ iv

Table of Contents $\quad$ vi

List of Tables $\quad$ xi

List of Figures $\quad$ xiv

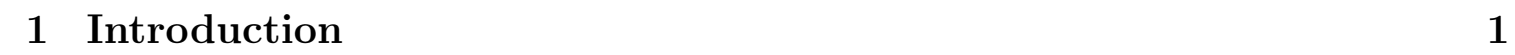

1.1 Outline . . . . . . . . . . . . . . . . . . . . . . . . 2

1.2 Author's Contributions to This Thesis . . . . . . . . . . . . . . . . . 2

1.3 Motivation . . . . . . . . . . . . . . . . . . 3

1.3 .1 The Standard Model . . . . . . . . . . . . . . . . . . . . . . . 3

1.3.2 Dark Matter . . . . . . . . . . . . . . . . . . . . . . 6

1.3 .3 Strong CP Problem . . . . . . . . . . . . . . . . . 15

\begin{tabular}{|lll|}
\hline 2 & $5.5 \mathrm{MeV}$ Solar Axion Theory & 25
\end{tabular}

2.1 Rate of $5.5 \mathrm{MeV}$ Axion Production . . . . . . . . . . . . . . . . . . . 25

2.1 .1 Selection Rules . . . . . . . . . . . . . . . . 25

2.1 .2 Solar Axion Production Mechanism . . . . . . . . . . . . . . . 26

$2.2 \quad$ Axion Interaction Cross Sections . . . . . . . . . . . . . . . . . . . . 30 
$2.2 .1 \quad$ Axion Decays in Transit from the Sun . . . . . . . . . . . . 36

$2.3 \quad$ Axion Escape from the Sun . . . . . . . . . . . . . . . . . . 37

2.4 Rates of Interactions in the Detector . . . . . . . . . . . . . . . . . . 39

\begin{tabular}{|lll}
3 & The DEAP-3600 Experiment & 42
\end{tabular}

$3.1 \quad$ Detector Design $\ldots \ldots \ldots$. . . . . . . . . . . . . . . . . . . . . . . . . . . 43

3.2 Signal and Signal Detection . . . . . . . . . . . . . . . 46

3.3 PMT and Digitizer Saturation . . . . . . . . . . . . . . 51

3.4 Datasets . . . . . . . . . . . . . . . . . . 55

3.4 .1 AmBe Calibration Data . . . . . . . . . . . . . 55

3.4 .2 Low Background ("Physics") Data . . . . . . . . . . . . . . . . 58

3.5 Software . . . . . . . . . . . . . . . . . . . . . . 59

4 Radioactivity in DEAP-3600 60

$4.1 \quad \alpha$ Decay Backgrounds . . . . . . . . . . . . . . . . . . . . . 60

4.2 Radiogenic Neutrons . . . . . . . . . . . . . . . . . . . . . . . . . 64

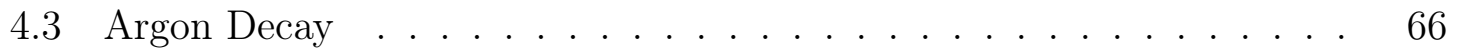

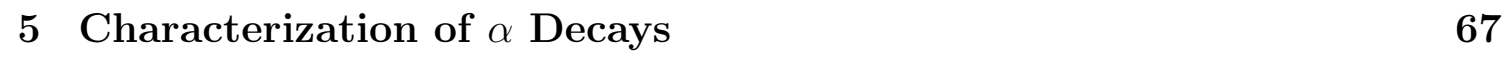

5.1 Cut Variables . . . . . . . . . . . . . . . . . . . . . . . . . . . . . 68

5.2 Determining the Activities of ${ }^{222} \mathrm{Rn},{ }^{218} \mathrm{Po},{ }^{214} \mathrm{Po},{ }^{220} \mathrm{Rn},{ }^{216} \mathrm{Po}, \&{ }^{212} \mathrm{Bi} \quad 69$

5.2 .1 Introduction . . . . . . . . . . . . . . . . . . . 69

5.2 .2 Overview of approach. . . . . . . . . . . . . . . . . . . . 72

$5.2 .3 \quad$ Detailed Description of Energy Correction . . . . . . . . . . . 74

5.2 .4 Detailed description of fitting methods . . . . . . . . . . . 78

5.2 .5 Results . . . . . . . . . . . . . . . . . . . . . 82

5.2 .6 Systematics Study . . . . . . . . . . . . . . 86

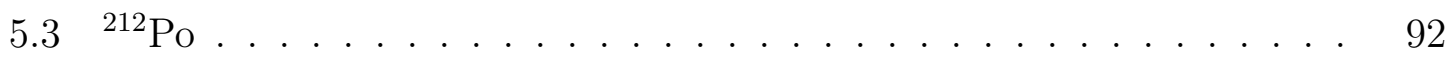


5.3 .1 Cuts $\ldots \ldots \ldots \ldots \ldots \ldots$

5.3 .2 Result . . . . . . . . . . . . . . . . . . . . . . . . . . 94

$5.3 .3{ }^{212} \mathrm{Po}_{\text {Systematics }} \ldots \ldots \ldots \ldots \ldots$

$5.4 \quad$ Time Coincidence Tagging of ${ }^{222} \mathrm{Rn}-{ }^{218} \mathrm{Po},{ }^{220} \mathrm{Rn}-{ }^{216} \mathrm{Po}$, and ${ }^{214} \mathrm{Bi}-$

${ }^{214} \mathrm{Po} \ldots \ldots \ldots \ldots$

5.4 .1 Introduction . . . . . . . . . . . . . . . . . . . . 99

5.4 .2 Tagging ${ }^{214} \mathrm{Bi}$ and ${ }^{214} \mathrm{Po} \ldots \ldots \ldots \ldots \ldots$

5.4 .3 Tagging ${ }^{220} \mathrm{Rn}$ and ${ }^{216} \mathrm{Po} \ldots \ldots \ldots \ldots \ldots$

5.4 .4 Tagging ${ }^{222} \mathrm{Rn}$ and ${ }^{218} \mathrm{Po} \ldots \ldots \ldots \ldots \ldots$

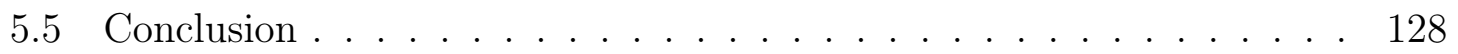

\begin{tabular}{|lll}
6 & Estimation of the Radiogenic Neutron Background & 134
\end{tabular}

6.1 Tagging Method . . . . . . . . . . . . . . . . . . . . . . . . 134

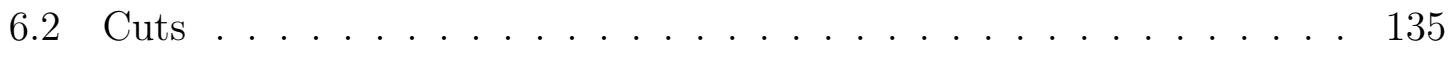

6.3 Time Window . . . . . . . . . . . . . . . . . . . . . . . . . . 137

6.4 Tagging Efficiency $\ldots \ldots \ldots \ldots \ldots$

6.5 Monte Carlo . . . . . . . . . . . . . . . . . . . . . . . . . . 142

6.6 Results . . . . . . . . . . . . . . . . . . . . . . . . . . . 146

6.6 .1 Uncertainties . . . . . . . . . . . . . . . . . . . . . 148

6.7 Tagging Cut . . . . . . . . . . . . . . . . . . . . . . . 148

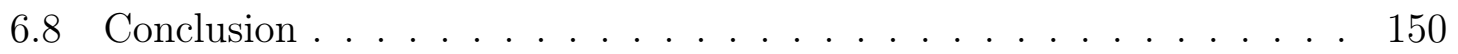

\begin{tabular}{|lll}
\hline 7 & Search for 5.5 MeV Solar Axions & 152
\end{tabular}

7.1 Expected Signal and Background $\ldots \ldots \ldots \ldots$

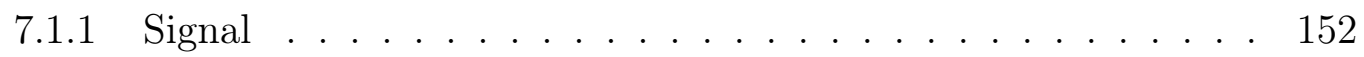

7.1 .2 Backgrounds in Model . . . . . . . . . . . . . . . . . . 154

7.2 Overview of Procedure $\ldots \ldots \ldots \ldots \ldots \ldots$

7.2 .1 Blinding and Unblinding Procedure . . . . . . . . . . 157 
7.3 Generating Monte Carlo Datasets . . . . . . . . . . . . . . 157

$7.3 .1 \quad$ AmBe MC Generator and Geometry . . . . . . . . . . . 158

7.3 .2 Axion MC Generators . . . . . . . . . . . . . . . 160

7.4 Processing Simulation Output . . . . . . . . . . . . . . . . . . 164

7.4.1 Extracting Physics Process and Isotope Information . . . . . . 164

7.4.2 Processing Energy Depositions into Simulated Triggers . . . . 166

$7.4 .3 \quad$ Monte Carlo Neutron Capture $\gamma$ Spectrum . . . . . . . . . . . 167

$7.5 \quad$ Fitting $\ldots \ldots \ldots \ldots \ldots \ldots \ldots \ldots \ldots \ldots \ldots \ldots \ldots \ldots \ldots$

$7.5 .1 \quad$ Energy response function $\ldots \ldots \ldots \ldots \ldots$

7.5 .2 Fit Overview . . . . . . . . . . . . . . . . . . . 168

7.5 .3 BAT and MCMC fitting intro . . . . . . . . . . . . . 169

7.6 Corrections to Simulated Model . . . . . . . . . . . . . . . . . 178

7.6.1 Separating Model into Capture- $\gamma$ Spectral Components . . . . 178

7.6 .2 Corrections to the Neutron Capture- $\gamma$ Spectra . . . . . . . . 180

7.6 .3 Energy Conservation in MC . . . . . . . . . . . . . . 187

7.7 Cuts and Data Corrections . . . . . . . . . . . . . . . . 188

$7.7 .1 \quad$ Cuts . . . . . . . . . . . . . . . . . . 188

$7.7 .2 \quad$ LY correction . . . . . . . . . . . . . . . . . . . 190

7.7 .3 Background subtraction . . . . . . . . . . . . . . . . 192

7.7 .4 Saturation Correction. . . . . . . . . . . . . . . . 195

7.8 Detector Datasets . . . . . . . . . . . . . . . . . . . . . . . 195

7.8 .1 Making the AmBe Calibration Dataset . . . . . . . . . . 195

7.9 Fit Validation with Toy Datasets $\ldots \ldots \ldots \ldots \ldots$

7.9 .1 Toy Dataset Generation . . . . . . . . . . . . . . . . . . 199

7.9 .2 Toy Physics Fits $\ldots \ldots \ldots$

7.9 .3 Toy AmBe Fits . . . . . . . . . . . . . . . . . . . . . . 201

7.9 .4 Sensitivity Studies . . . . . . . . . . . . . . . . . . 202 
7.10 Final Blinded Fits. . . . . . . . . . . . . . . . . . . . . 203

7.10 .1 Final AmBe Fit . . . . . . . . . . . . . . . . . . . . 203

7.10 .2 Final Blinded Physics Fit . . . . . . . . . . . . . 203

7.10 .3 Effect of Systematic Uncertainties . . . . . . . . . . . . 204

7.11 Exclusion Curves . . . . . . . . . . . . . . . . . . . . . . 205

7.11.1 Projected Exclusion Curves Prior to Unblinding . . . . . . . . 205

7.12 Unblinding and Excess in Physics Side-band . . . . . . . . . 206

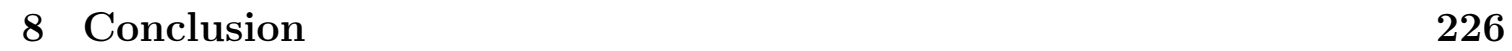

\begin{tabular}{ll}
\hline References & 229
\end{tabular}

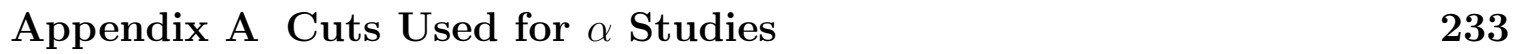

\begin{tabular}{|lll}
\hline Appendix B Fits for $\alpha$ Systematics Study & 237
\end{tabular}

\begin{tabular}{|lll}
\hline Appendix C & Correlation Matrices for 5.5 MeV Solar Axion Search 257
\end{tabular} 


\section{List of Tables}

$2.1 \quad$ Axion couplings allowed if axions escape from Sun . . . . . . . . . . . 39

3.1 Event locations in $\mathrm{qPE}$ and fprompt . . . . . . . . . . . . . . . . . 52

4.1 Types of events caused by $\alpha$ decays . . . . . . . . . . . . . . . . . . . 60

5.1 Number of $\alpha$ decays generated for fit $\ldots \ldots$. . . . . . . . . 73

5.3 Correlation matrix for fit in Figure 5.7 . . . . . . . . . . . . . . . . . 84

$5.2 \quad$ LAr bulk activities for $\mathrm{U}$ and Th chain isotopes. . . . . . . . . . . . . 84

5.4 LAr bulk activities for U and Th chain isotopes with combined errors 85

5.5 Number of events in "box cut" regions . . . . . . . . . . . . . . . . 85

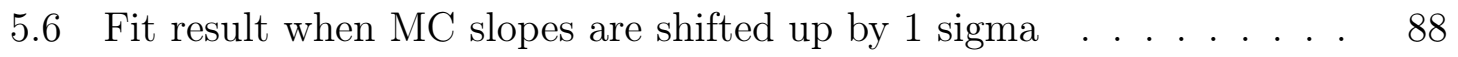

$5.7 \quad$ Fit result when MC slopes are shifted down by 1 sigma . . . . . . . . 89

$5.8 \quad$ Fit result when MC intercepts are shifted up by 1 sigma . . . . . . . 89

$5.9 \quad$ Fit result when MC intercepts are shifted down by 1 sigma . . . . . . 90

5.10 Fit result when resolution term is shifted up by 1 sigma . . . . . . . . 90

5.11 Fit result when resolution term is shifted down by 1 sigma . . . . . . 91

5.12 Counts of ${ }^{212} \mathrm{Po}$ candidates . . . . . . . . . . . . . . 95

5.13 Numbers of tagged high fprompt events in various qPE ranges . . . . 104

5.14 Efficiencies of cuts used to identify degraded surface events . . . . . . 114

5.15 Count of tags in signal and background window for various qPE ranges 114

5.16 Surface candidates in various qPE ranges corrected for efficiencies . . 115

5.17 List of cuts applied to data in order to identify neck $\alpha$ events. . . . . 116 
5.18 Numbers of tagged and untagged neck events . . . . . . . . . . . . . 118

6.1 Summary of radiogenic neutron results . . . . . . . . . . . . . . . 147

6.2 Ratios of MC neutrons in blinding regions to MC neutrons in CR . . 147

6.3 Expected numbers of radiogenic neutrons in blinding strips and ROI. 148

6.4 Breakdown of uncertainty on final result for ROI. . . . . . . . . . . . 150

6.5 Expected numbers of radiogenic neutrons after neutron tag cut . . . . 151

7.1 Descriptions of components in fits . . . . . . . . . . . . . . . . . . . 208

7.2 Fraction of toy physics data fits with correct mean number of axions. 208

7.3 Fraction of $68 \%$ confidence intervals that include correct parameter value 210

7.4 Sensitivity of axion search for different axion signal strength $\langle\mathrm{N}\rangle$. . 210

7.5 Complete AmBe calibration fit results . . . . . . . . . . . . . . . . 216

7.6 Table of selected correlations in the AmBe calibration fit . . . . . . . 218

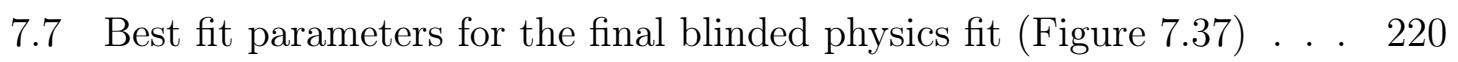

7.8 Table of selected correlations in the blinded physics fit . . . . . . . . 221

7.9 Physics fit results with the Mn normalization fixed to 2 . . . . . . . . 223

B.1 Fit result when MC slopes are shifted up by 1 sigma $\ldots . . . .238$

B.2 $\quad$ Fit result when MC slopes are shifted up by 1 sigma $\ldots . . . .238$

B.3 $\quad$ Fit result when MC slopes are shifted down by 1 sigma . . . . . . . . 239

B.4 Fit result when MC slopes are shifted down by 1 sigma . . . . . . . . 240

B.5 Fit result when MC intercepts are shifted up by 1 sigma . . . . . . . 241

B.6 $\quad$ Fit result when MC intercepts are shifted up by 1 sigma . . . . . . . 242

B.7 $\quad$ Fit result when MC intercepts are shifted down by 1 sigma . . . . . . 243

B.8 $\quad$ Fit result when MC intercepts are shifted down by 1 sigma . . . . . . 244

B.9 $\quad$ Fit result when $\mathrm{MC}$ qPE is shifted up $0.2 \%$ after calibration . . . . . 246

B.10 Fit result when $\mathrm{MC} q \mathrm{PE}$ is shifted up $0.2 \%$ after calibration . . . . . 246

B.11 Fit result when MC qPE is shifted down $0.2 \%$ after calibration. . . . . 248

B.12 Fit result when MC qPE is shifted down $0.2 \%$ after calibration . . . . 248 
B.13 Fit result when MC fprompt is shifted up 0.2\% after calibration . . . 250

B.14 Fit result when MC fprompt is shifted up $0.2 \%$ after calibration . . . 250

B.15 Fit result when MC fprompt is shifted down $0.2 \%$ after calibration. . 252

B.16 Fit result when MC fprompt is shifted down $0.2 \%$ after calibration. . 252

B.17 Fit result when resolution term is shifted up by 1 sigma. . . . . . . . 253

B.18 Fit result when resolution term is shifted up by 1 sigma. . . . . . . . . 254

B.19 Fit result when resolution term is shifted down by 1 sigma . . . . . . 255

B.20 Fit result when resolution term is shifted down by 1 sigma . . . . . . 256 


\section{List of Figures}

1.1 The elementary particles included in Standard Model . . . . . . . . . 4

1.2 Rotation curve of matter within Messier 33 . . . . . . . . . . . . . . . 7

1.3 A Hubble Space Telescope image of the Cl $0024+17$ galaxy cluster . . $\quad 9$

1.4 Composite image of the Bullet Cluster . . . . . . . . . . . . . . 10

1.5 CMB multipole moments . . . . . . . . . . . . . . . . . . . . . . . . . 12

$1.6 \quad$ Fundamental axion Feynman diagrams . . . . . . . . . . . . . . . . . 24

$2.1 \omega_{A} / \omega_{\gamma}$ for hadronic axions. . . . . . . . . . . . . . . . 29

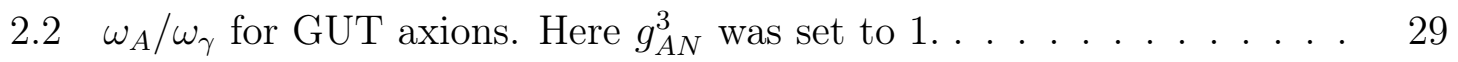

2.3 Axion cross sections of interest for this work . . . . . . . . . . . . . . 31

2.4 1 loop order coupling of axion to electron . . . . . . . . . . . . . . . . 32

2.5 The axion Compton conversion cross section for DEAP-3600 . . . . . 33

$2.6 \quad \gamma$ and electron energy distributions in Compton conversion . . . . . . 34

2.7 The inverse Primakov conversion cross section for DEAP-3600 . . . . 35

2.8 The axio-electric effect cross section in DEAP-3600 for $g_{A} e=1$. . . . 36

2.9 Axio-electric effect cross section for iron with $g_{A e}=1$ for $5.5 \mathrm{MeV}$ axions. 38

2.10 The number of axion decays to two photons expected in DEAP-3600 40

2.11 Number of Primakov conversions on argon in DEAP-3600 . . . . . . . 41

3.1 Cross-section of the DEAP-3600 detector . . . . . . . . . . . . . . 44

$3.2 \quad$ A sample waveform for a $\gamma$ absorption in DEAP's liquid argon volume 48

$3.3 \quad$ A sample waveform for a neutron scatter within DEAP . . . . . . . . 49 
3.4 The distribution of LAr scintillation events in DEAP-3600 . . . . . . 51

$3.5 \quad$ Illustration of DEAP DAQ system . . . . . . . . . . . . . . . . 53

$3.6 \quad$ Sample waveforms for $\alpha$ particle scatters in liquid argon. . . . . . . . . 54

3.7 Sample Monte Carlo generated PMT hit patterns . . . . . . . . . . . 54

$3.8 \quad \alpha$ decay peaks in fprompt vs qPE . . . . . . . . . . . . . . . 55

3.9 Image of the DEAP-3600 detector after being encased in the steel shell 56

3.10 Energy level diagram of the ${ }^{12} \mathrm{C}$ nucleus . . . . . . . . . . . . . . . . 58

$4.1{ }^{238} \mathrm{U}$ decay chain $\ldots \ldots \ldots$. . . . . . . . . . . . . . 61

$4.2{ }^{232}$ Th decay chain . . . . . . . . . . . . . . . . . . . . . . . 62

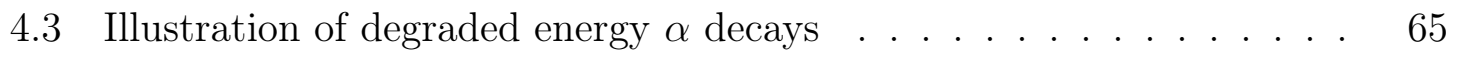

5.1 Raw $\alpha$ Monte Carlo and data qPE distributions . . . . . . . . . . . . 70

$5.2 \quad$ Monte Carlo and data fprompt vs qPE distributions before fitting . . $\quad 72$

5.3 The $\alpha$ region in fprompt vs qPE, with the "box cuts" overlaid . . . . 74

5.4 Lines fit to the Monte Carlo $\alpha$ populations to correct clipping . . . . 76

$5.5 \quad$ Slopes of lines from fits to Monte Carlo and data $\alpha$ distributions . . . $\quad 77$

$5.6 \quad$ Intercepts of lines fit to Monte Carlo and data $\alpha$ distributions . . . . 78

$5.7 \quad$ Best fit of the corrected MC energy spectrum to the corrected $\alpha$ data $\quad 82$

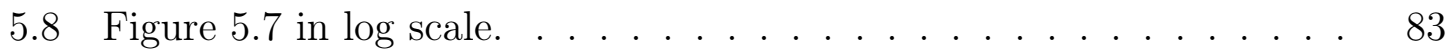

$5.9 \quad$ Monte Carlo of ${ }^{212} \mathrm{Po}$ and nearby $\alpha$ populations..... .93

5.10 Data events in the ${ }^{214} \mathrm{Po}$ and ${ }^{212} \mathrm{Po}$ region . . . . . . . . . . . . . . . 94

5.11 Cut used in computing central value of ${ }^{212} \mathrm{Bi}$ decay count estimate. . $\quad 96$

5.12 Shifted up $500 \mathrm{qPE} 60$. . . . . . . . . . . . . . . . . . 97

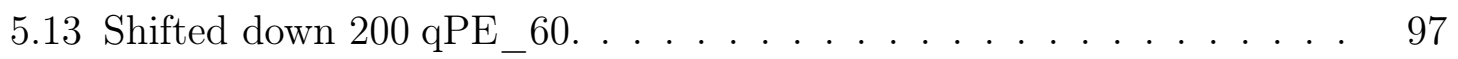

5.14 Slope increased. . . . . . . . . . . . . . . . . . . . . . . 98

5.15 Slope decreased. . . . . . . . . . . . . . . . . . . . . . . . . . 98

5.16 Sample spectrum of the $\mathrm{Bi}^{214}$ Po coincidence tag time separation . . . 100

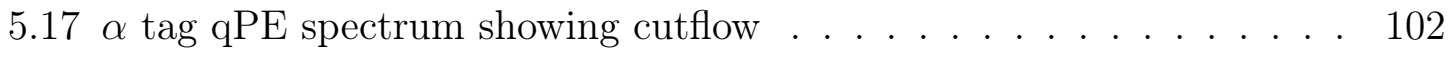


5.18 Same as Figure 5.17, but zoomed-in to WIMP ROI-scale energies. . . 103

5.19 All data events after standard low level and pileup cuts . . . . . . . . 104

5.20 fprompt vs qPE distribution of tagged ${ }^{214}$ Po events . . . . . . . . . . 105

5.21 Distribution of the time between tagged pairs for ${ }^{214}$ Po bulk events . 106

5.22 The tagging efficiency of MC ${ }^{214} \mathrm{Po}$ events in the LAr vs qPE . . . . . 107

5.23 The tagging efficiency of data ${ }^{214} \mathrm{Po}$ events in the LAr vs qPE . . . . 107

5.24 Tag time separation where the high fprompt tag has $\mathrm{qPE}<100$. . . 109

5.25 Tag time separation with high fprompt tag $100<\mathrm{qPE}<{ }^{214} \mathrm{Po}$ Peak $\quad 110$

5.26 Time between $\mathrm{Bi}^{214} \mathrm{Po}$ tags with $100<\mathrm{qPE}<{ }^{214} \mathrm{Po}$ Peak $\ldots$. . . . . 111

5.27 Distribution of spatial separation between time-coincidence tags . . . 111

5.28 PMT map showing the efficiencies of the PMTs on a colour scale . . . 112

5.29 Monte Carlo tagging efficiency for $\mathrm{Bi}^{214} \mathrm{Po}$ events . . . . . . . . . . . 115

5.30 Diagram (from $|1|$ ) showing the flow guide geometry in the neck. . . . 117

5.31 fprompt vs qPE of simulated inner flowguide $\mathrm{Bi}^{214}$ Po events . . . . . 118

5.32 Neck events in mblikelihoodZ vs qPE . . . . . . . . . . . . . . . . . . 119

5.33 Simulated $\mathrm{Bi}^{214} \mathrm{Po}$ events in mblikelihoodZ vs qPE overlaid on data . 120

5.34 The qPE distribution of data and Monte Carlo neck events . . . . . . 121

5.35 fmaxpe vs qPE for data, showing potential dust distribution . . . . . 122

5.36 Data events in fprompt vs qPE space, showing low fmaxpe events . . 122

$5.37 \alpha$ fmaxpe distributions $\ldots \ldots \ldots$. . . . . . . . . . . . . 123

$5.38 \alpha$ mblikelihoodR distributions . . . . . . . . . . . . . . . . . . . . . . 124

5.39 Locations of low fmaxpe degraded $\alpha$ events . . . . . . . . . . . . . . . 125

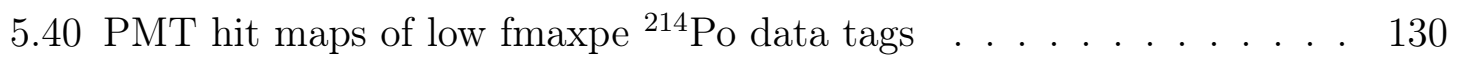

5.41 Sample PMT hit maps of ${ }^{210}$ Po surface events in data . . . . . . . . . 131

5.42 fprompt vs qPE of $\alpha$ - $\alpha$ tags in the data within a $0.5 \mathrm{~s}$ window . . . . 131

5.43 Spatial tag separation cut for $\alpha$ - $\alpha$ data tags . . . . . . . . . . . . . 132

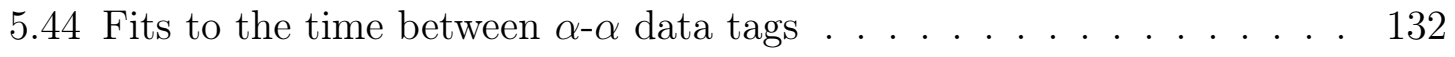


5.45 Locations of $\alpha$ - $\alpha$ tags and time fit of low fmaxpe tags . . . . . . . . 133

6.1 Illustration of a radiogenic neutron scatter and capture . . . . . . . . 135

6.2 Acceptance vs MBR for ${ }^{39} \mathrm{Ar}$ data (run 21399) and MC . . . . . . . . 137

6.3 The distribution of the time between tagged pairs . . . . . . . . . . . 139

$6.4 \quad$ Fit of the time between neutron scatter and capture candidate tags . 139

6.5 Tagging efficiency across several runlists . . . . . . . . . . . . . . . . 141

6.6 MC neutron recoils in LAr for some of the radiogenic neutron sources 143

6.7 Tagged radiogenic neutron candidates in fprompt vs qPE . . . . . . . 149

7.1 Total energy deposited in the detector after an axion interaction . . . 153

7.2 Total energy deposited in the detector for different axion masses . . . 154

7.3 Fit of the DEAP-3600 electron-recoil spectrum (by a collaborator) . . 155

7.4 Illustration of the production mechanism of the neutron capture $\gamma$. . 155

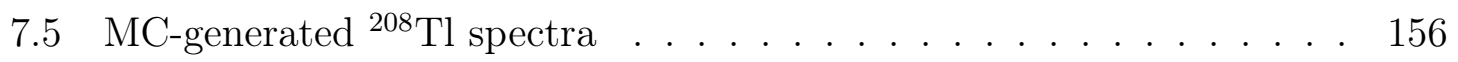

7.6 AmBe source encapsulation photos . . . . . . . . . . . . . . . 160

7.7 The simulated encapsulated AmBe source. . . . . . . . . . . . . . . . 161

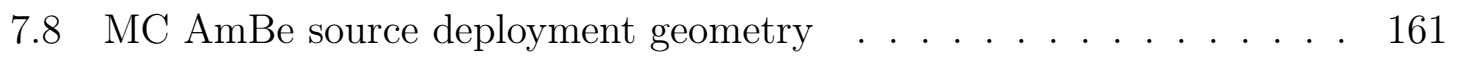

7.9 The four axion interaction cross sections under study . . . . . . . . . 162

7.10 Initial AmBe fit with only response function free to float . . . . . . . 179

7.11 Same as Figure 7.10 , but with the neutron capture- $\gamma$ spectra overlaid. 180

7.12 AmBe fit with neutron capture- $\gamma$ spectra by element . . . . . . . . . 181

7.13 Comparisons of GEANT4 vs ENDF neutron capture- $\gamma$ spectra . . . . 182

7.14 Sample neutron capture- $\gamma$ spectra after corrections . . . . . . . . . . 184

7.15 AmBe fit after spectral corrections . . . . . . . . . . . . . . . 185

7.16 Illustration of spectral systematic variations . . . . . . . . . . . . . 186

7.17 The $5.5 \mathrm{MeV}$ electron signal sensitivity . . . . . . . . . . . . . . . . . 189

7.18 The $5.5 \mathrm{MeV} \gamma$ (Primakov) signal sensitivity . . . . . . . . . . . . . 190

7.19 Radial cut acceptances . . . . . . . . . . . . . . . . . . 191 
7.20 Plot used to calculate MC radial cut . . . . . . . . . . . . . 191

7.21 The DEAP-3600 light yield as a function of data run number . . . . . 192

7.22 Effect of light yield correction on fit . . . . . . . . . . . . . . 193

7.23 AmBe background subtraction . . . . . . . . . . . . . . . . 194

7.24 Effect of saturation correction on fit . . . . . . . . . . . . . . 196

7.25 The fraction of the physics dataset live-time in 1 month wide bins. . . 197

7.26 Dates of AmBe data used in fits . . . . . . . . . . . . . . 198

7.27 Sample toy physics data fit, selected at random, with $\langle\mathrm{N}>=0.2$. . 200

7.28 Sample toy physics data fit, selected at random, with $<\mathrm{N}>=15$. . . 201

7.29 Sample toy physics data fit, selected at random, with $\langle\mathrm{N}>=30$. . . 202

7.30 Distributions of toy physics fit results . . . . . . . . . . . . . 209

7.31 Distributions of toy Ambe energy scale fit results $\ldots \ldots \ldots \ldots . . .211$

7.32 Distributions of toy Ambe resolution parameter fit results. . . . . . . 212

7.33 Samples of toy AmBe data fits, selected at random. . . . . . . . . 213

7.34 The distributions of 90\% CL upper limits derived from toy dataset fits 214

7.35 Final AmBe fit . . . . . . . . . . . . . . . . . . . . . 215

7.36 Fully marginalized posteriors from the AmBe calibration fit. . . . . . 217

7.37 Final physics fit . . . . . . . . . . . . . . . . . . . . . 219

7.38 Fully marginalized posteriors from the blinded physics fit. . . . . . . 221

7.39 Physics fit with the Mn normalization fixed to 2 . . . . . . . . . 222

7.40 Effects of nuisance parameters on axion component uncertainty . . . 224

7.41 Projected DEAP exclusions . . . . . . . . . . . . . . . 225

B.1 Fit result when MC slopes are shifted up by 1 sigma $\ldots \ldots \ldots . . .237$

B.2 $\quad$ Fit result when MC slopes are shifted down by 1 sigma . . . . . . . . 239

B.3 $\quad$ Fit result when MC intercepts are shifted up by 1 sigma . . . . . . . 241

B.4 Fit result when MC intercepts are shifted down by 1 sigma . . . . . . 243

B.5 Fit result when MC qPE is shifted up $0.2 \%$ after calibration . . . . 245 
B.6 $\quad$ Fit result when MC qPE is shifted down $0.2 \%$ after calibration . . . . 247

B.7 $\quad$ Fit result when MC fprompt is shifted up 0.2\% after calibration . . . 249

B.8 Fit result when MC fprompt is shifted down $0.2 \%$ after calibration. . 251

B.9 $\quad$ Fit result when resolution term is shifted up by 1 sigma . . . . . . . . 253

B.10 Fit result when resolution term is shifted down by 1 sigma . . . . . . 255

C.1 Correlation matrix for the full AmBe calibration fit . . . . . . . . . . 258

C.2 Correlation matrix for the blinded physics fit . . . . . . . . . . . . . . 259 


\section{Chapter 1}

\section{Introduction}

DEAP-3600 is a dark matter search experiment located over $2 \mathrm{~km}$ underground in Sudbury, Ontario, Canada, searching for Weakly Interacting Massive Particles (WIMPs). DEAP-3600's sensitive volume is an acrylic sphere filled with over 3 tonnes

of liquid argon (LAr) [2]. When a particle enters the LAr, it can recoil off one of the argon atoms, which results in scintillation light being produced in the argon. The sphere is surrounded by 255 photo-multiplier tubes (PMTs), which collect the scintillation light from particle interactions.

An essential requirement of the experiment is that the level of radioactivity within the detector be as low as possible. This is especially true for $\alpha$ decays and neutron events within the liquid argon target material, as these can mimic WIMP events. To understand these backgrounds, several studies were undertaken, and are detailed in this thesis.

In addition to the primary goal of searching for WIMP interactions, DEAP-3600 is also capable of searching for other theoretical particle interactions. Among these are interactions from $5.5 \mathrm{MeV}$ solar axions. A full axion search analysis is detailed in this report. 


\subsection{Outline}

This thesis will start with a brief introduction to dark matter in section 1.3.2, including observational motivations for the theory of dark matter, some of the more popular theoretical dark matter models, and the properties of WIMP dark matter. This will be followed in Chapter 2 by an introduction to axions, covering the Strong CP Problem, the axion solution to this problem, axion production, and the properties of axions and their potential generation within the sun. I will then introduce the DEAP-3600 experiment in Chapter 3 and discuss some of the $\alpha$ and neutron related backgrounds in Chapter 4 .

These introductory chapters will be followed by Chapter 5, which will discuss my efforts to measure and understand the $\alpha$ decay background in DEAP-3600. Chapter 6 will discuss my estimates of the rate of background events in DEAP-3600 due to radiogenic neutron interactions with the detector. Finally, Chapter 7 will detail the search for evidence of interactions of 5.5 MeV solar axions in DEAP-3600.

\subsection{Author's Contributions to This Thesis}

The plots in the theory section relating to the the axion signal in DEAP-3600 specifically were created by me. The work in Chapter 5 was all my own, apart from the development of neck $\alpha$ selection cuts in table 5.17 and figure 5.32 showing the distribution of neck events in data. The $\alpha$ work was published in [1. As stated in Chapter 6, my work on radiogenic neutrons was based on previous work done by my

collaborators. None of the work that is referenced in Chapter 6 as being done by a previous study is mine, but all the work done by the "current" study was done by me. All the work described in Chapter 7 was done by me.

In addition to the work described in this report, the author served as the DEAP 
database co-administrator for one year, took detector data throughout the last 5 years (2016-2021), performed several position reconstruction studies, and contributed to the development of the DEAP software package.

\subsection{Motivation}

\subsubsection{The Standard Model}

The elementary particles described by particle physics fit neatly together in a mathematical structure known as the "Standard Model" of particle physics. All particles that have been detected to date are either included in this model themselves, or are made up of particles that are included in it. The Standard Model relates all the known fundamental particles to each other and to the fundamental interactions (although gravity is not always included here). The known particles are displayed in a table in Figure 1.1. The fundamental interactions are the electroweak interaction, strong nuclear interaction, gravity, and the Higgs interaction. Each interaction is mediated by one or more distinct particles, all of which are bosons, which are defined as having integer spin. When acting as mediators, they are known as "virtual" particles, because they only exist while the interaction is occurring. Virtual particles must exist

within the constraints of the Heisenberg uncertainty principle: $\Delta E \Delta t<\hbar[3]$. Under certain conditions, however, these particles can be produced as final state particles after an interaction is finished. These final state particles can then be detected by experiments. The other particles in the standard model are manifestations of different fields that exist throughout the universe. All particles are therefore manifestations of either the fields or the interactions between them.

Quarks are capable of interacting through any of the known fundamental interactions. These are only known to exist in nature in groups of two or more, known 


\section{Standard Model of Elementary Particles}

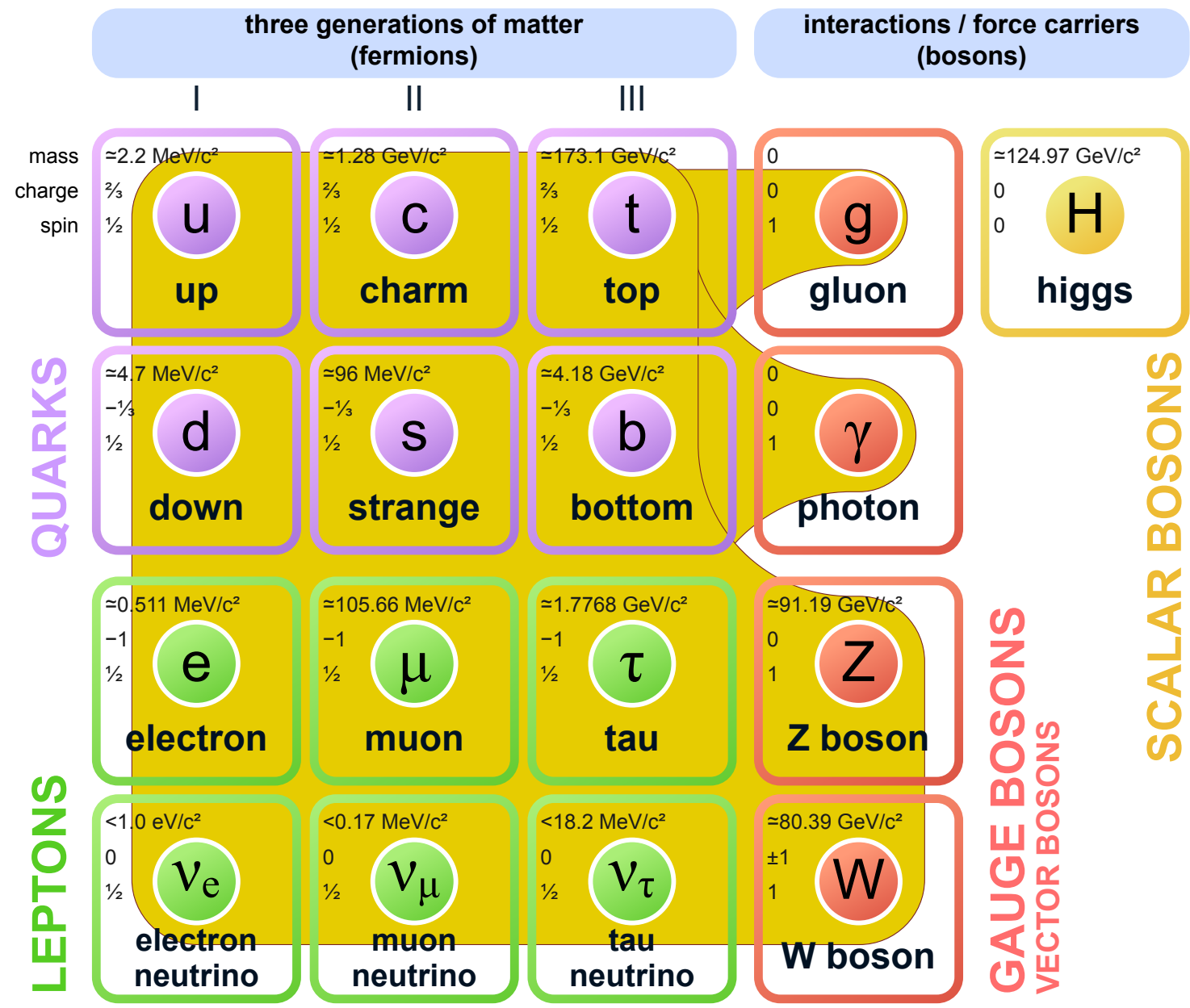

Figure 1.1: The elementary particles included in Standard Model of particle physics, along with their measured properties as of 2019. Image credit: Wikipedia 
as hadrons, due to the nature of the strong interaction [3]. Objects composed of an even number of quarks are known as mesons, and objects made up of an odd number of quarks are known as baryons. Protons and neutrons are the most well-known baryons.

Leptons are not capable of interacting through the strong interaction, and only the charged leptons can interact electromagnetically. The most well-known lepton is the electron. Neutrinos can only interact through the weak interaction and gravity, and are often produced when two particles interact via the weak interaction [3].

The photon, which we observe as light, is the mediator of the electromagnetic interaction. The gluons mediate the strong interaction, and have a very short range due to the nature of the strong interaction. The $\mathrm{W}$ and $\mathrm{Z}$ bosons mediate the weak interaction, and these too have a short range due to their large mass, which causes them to quickly decay to lighter particles whenever they are produced. All of these particles are known as gauge bosons, because they arise as the result of a symmetry known as gauge invariance [3]. The interactions they mediate, together with gravity, are often referred to as the fundamental forces of nature.

Lastly, the Higgs boson is in its own category, as it does not arise from gauge symmetry. It's existence is required in order for several of the other fundamental particles to have a mass, and is the carrier of the Higgs interaction.

Atoms are made up of protons, neutrons, and electrons. As shown in Figure 1.1 . electrons are the lightest charged lepton in the standard model. Protons and neutrons are each made up of combinations of three up and down quarks, which are baryons. Thus, all the elementary particles that make up atoms are charged, and, therefore, capable of interacting electromagnetically or through the strong interaction. This rules them out as dark matter, as electromagnetic interactions eventually result in the emission of light, and the strong interaction eventually results in either a bound state of atomic matter or in the creation of particles composed of two quarks [3], 
which eventually decay and produce light. One possible exception to this would be if these particles come together to form large-scale electrically neutral objects (such as asteroids, black holes, etc) scattered throughout the universe [4]. This theory, known as the MaCHO theory, will be discussed in the next section.

\subsubsection{Dark Matter}

Dark matter is currently one of the most interesting questions in particle physics research. Dark matter is thought to exist based on numerous astronomical observations, many of which are unrelated to one another. Not much about the nature of dark matter is known, however, as it has yet to be directly detected. It's existence is inferred based on its gravitational influence on the structure and formation of the universe, among other indirect evidences.

\section{Evidence for Dark Matter}

The first observations that hinted at the existence of dark matter were related to the movements of galaxies within the Coma Cluster [5], [6] and the velocities of stars as they moved around the centre of their galaxy [7], [8], [9], [10]. The mass of galaxies can be determined by plotting the rotation speed of luminous matter as a function of its distance from the centre of the galaxy to get what is known as a rotation curve. A relatively simple calculation involving Newton's Law of Gravitation and centripetal force is then used to find the total mass enclosed within a given radius, thus giving a profile of the mass distribution within a galaxy. An alternative way of measuring the distribution of mass within a galaxy (for non-dark matter) is to measure the luminosity of different regions of a given galaxy and compare that luminosity with empirical mass to luminosity $(\mathrm{M} / \mathrm{L})$ ratios.

As measurements became more accurate, it was evident that the shapes of these curves did not match what was predicted based on the observed locations of stars 


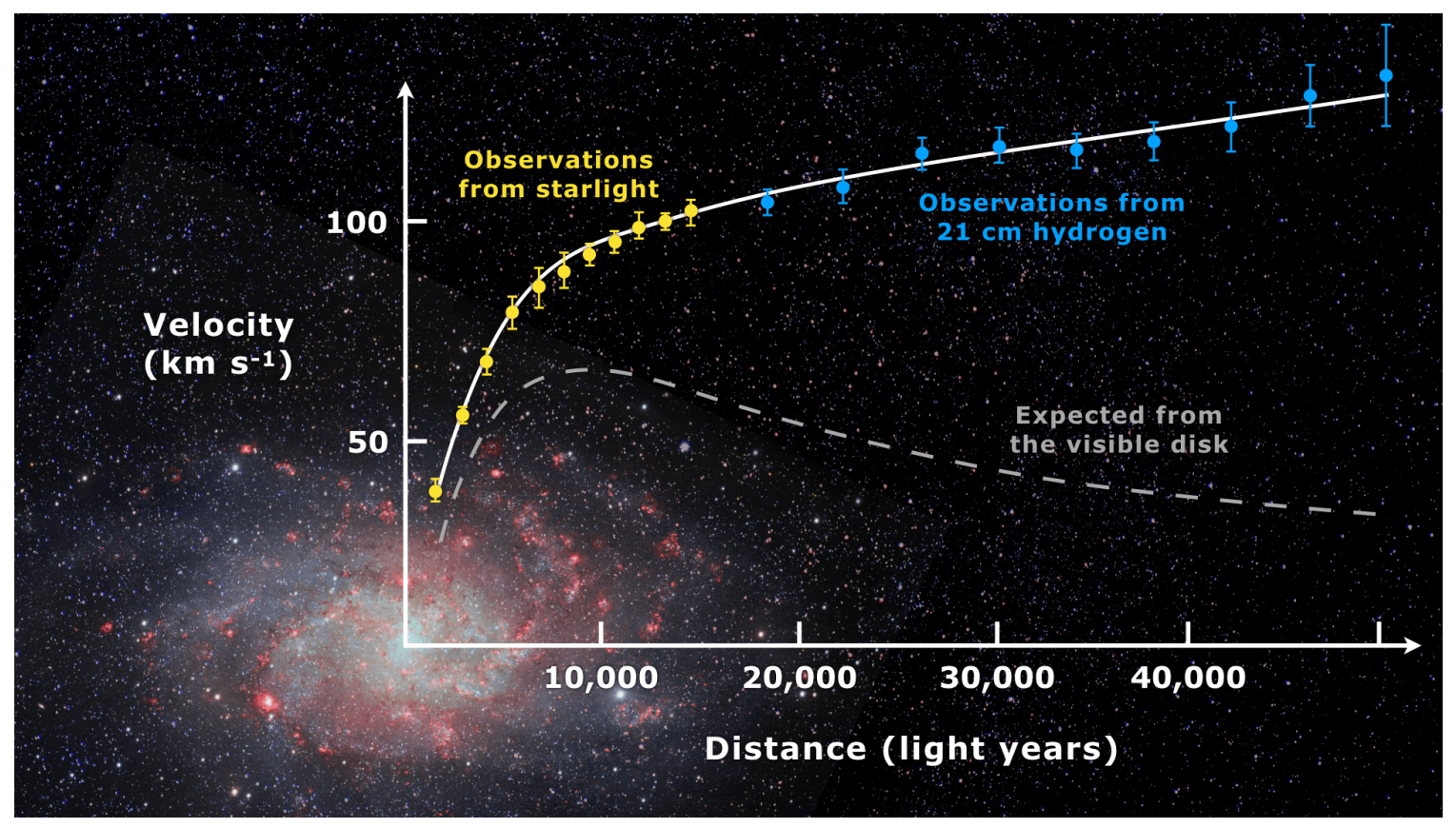

Figure 1.2: Rotation curve of matter within Messier 33 overlaid on an image of Messier 33. The curve shows a strong deviation of the observed velocities of stars and gas as a function of radius from that predicted from the gravitational influence of luminous matter in the galaxy. Image credit: Mario De Leo (Wikipedia). 
within the galaxy $|7|-[10$. In a typical galaxy, when one traces the density of stars outward from the centre of the galaxy, the density of stars decreases dramatically near the edge. Thus the mass density should also decrease. The relatively simple calculation involves equating the force from gravity to the centripetal force needed to keep the star in orbit around the galaxy is given below.

$$
\begin{aligned}
F=\frac{G m_{s} m_{e n c}}{r^{2}} & =\frac{m_{s} v^{2}}{r} \\
\frac{G m_{e n c}}{r} & =v^{2}
\end{aligned}
$$

where,

$$
m_{e n c}=\int_{0}^{r} 4 \pi \rho\left(r^{\prime}\right) r^{\prime 2} d r^{\prime}
$$

If the density is close to 0 at the edge, the plot of velocity vs distance from the centre will be proportional to $\frac{1}{\sqrt{r}}$. However, the rotation curves were observed to be "flat" towards the edges of galaxies, implying that the $m_{e n c}$ term (the total mass from the galactic centre to some radius $r$ ) must still be increasing even at the very edge of the galaxy where very few stars are present (see Figure 1.2, for example). This implied that much more mass must be present within galaxies than was estimated based on the luminosity of the galaxy.

The same effect can be observed in the rotations of galaxies around the centre of mass of galaxy clusters.

Theories ranging from a modification of Newtonian gravity to the presence of invisible "dark" matter have been proposed to explain the unexpected shapes of these rotation curves. However, this is not the only effect for which dark matter could provide an explanation.

General relativity predicts that gravity can bend the path of light, leading to an effect known as gravitational lensing. If an object with a large gravitational field sits between another luminous object and an observer, the light from the luminous 


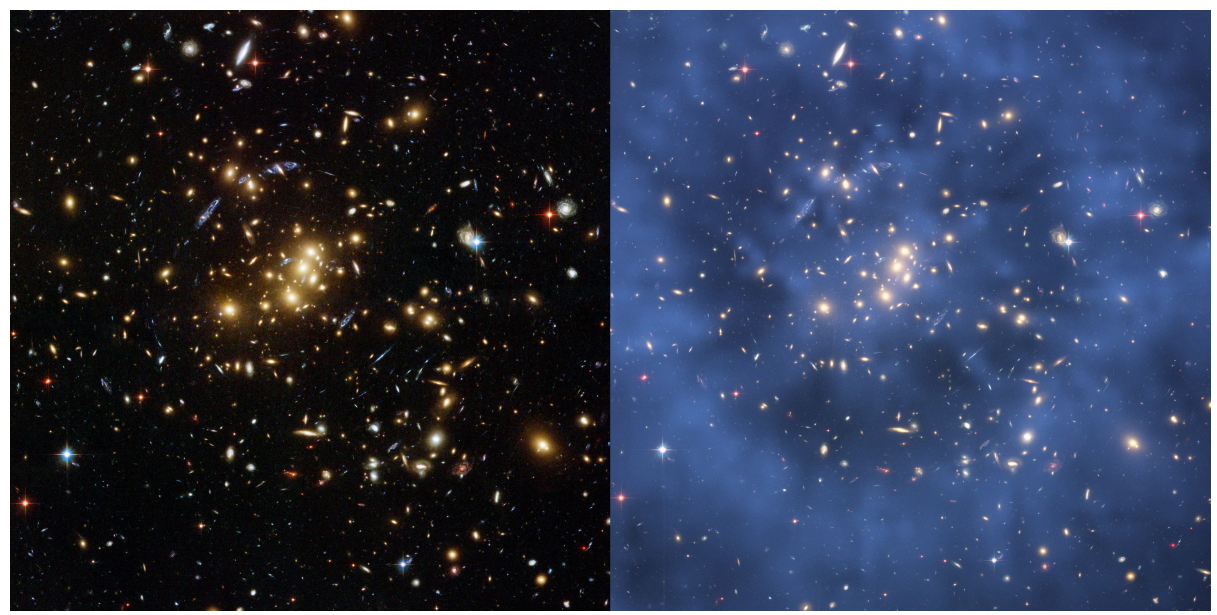

Figure 1.3: A Hubble Space Telescope image of the $\mathrm{Cl} 0024+17$ ( $\mathrm{ZwCl} 0024+1652)$ galaxy cluster. On the left is a visible spectrum image of the cluster in which magnified and distorted images of galaxies behind the cluster can be seen in the form of streaks around the centre of the image. This effect is the result of gravitational lensing. On the right is the same image overlaid with the distribution of invisible matter necessary to explain the observed gravitational lensing effect. (Image credit: NASA)

object gets redirected and focused, forming distorted images that are then seen by the observer. The path taken by the rays of light is analogous to the path of light as it gets redirected by a lens. This effect is referred to as "strong gravitational lensing", and is relatively rarely observed. On the other hand, "weak gravitational lensing", which is the distortion of the shapes of galaxies due the lensing effects, is observed throughout the universe. Through the application of statistical techniques to quantify the total amount of shape distortion, weak gravitational lensing effectively allows the mass density between earth and a collection of background objects to be mapped. This effect points to the presence of dark matter in galaxies (and other astronomical objects) on the same scale as predicted by the galaxy rotation curves. An example is shown in Figure 1.3 .

Gravitational lensing was also used in studying one particular case of two galaxies actively colliding - the Bullet Cluster (Figure 1.4 [11. Most baryonic ("normal") matter in a galaxy is in the form of stars and gas. The stars can be observed directly 


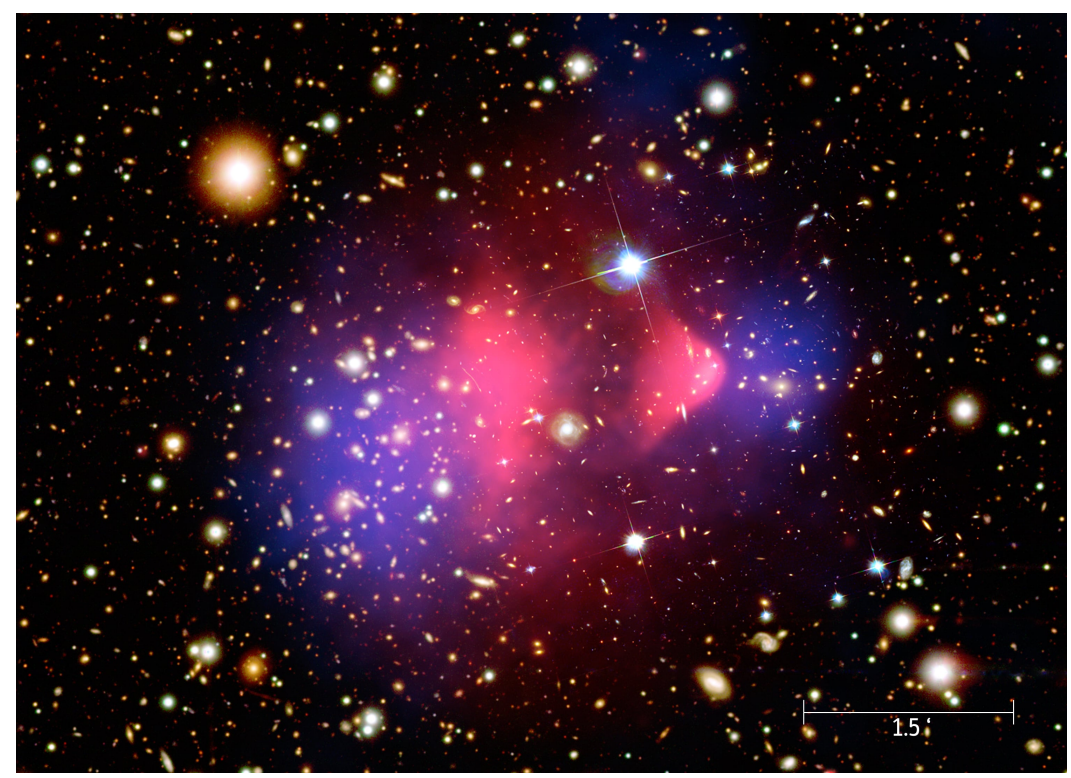

Figure 1.4: Composite image of the Bullet Cluster. The background image was taken in the visible spectrum by Magellan and the Hubble Space Telescope. The pink overlay is of the X-ray spectrum, taken by the Chandra Telescope. The blue is the mass distribution of the Bullet Cluster that is required to explain the observed gravitational lensing effects. (Image credit: NASA)

from the light they emit and the gas can be observed based on the X-rays they emit due to the energy released during the collision of the galaxies, as the gas from each galaxy interacted electromagnetically with the other. The total amount of normal matter can, therefore, be estimated. But when the total density of matter in the cluster was mapped using gravitational lensing, these observations showed that, although most of the normal, observable mass was in the hot gas, most of the mass measured with gravitational lensing was in the same region as the stars. This is thought to be due to the fact that the gas slowed down when it interacted electromagnetically, while the stars and dark matter passed through without such an interaction. In addition, computer models of the collision involving dark matter reproduce very well the mass distributions observed. The study of this cluster has provided some of the strongest evidence in support of dark matter rather than a modification of Newtonian gravity as the answer to these mysteries. 
In addition, information obtained from the Cosmic Microwave Background (CMB) further strengthen the case for dark matter. The CMB is the residual light left over from a time when the universe was in a state of thermal equilibrium among the various particles that existed at the time. As the universe expanded, atoms started to form in place of the sea of charged particles that were present before. Since atoms, which are neutral, scatter light much less than free charged particles, it was now possible for light to travel large distances without scattering. Some of the light leftover from the final moments of this transition from free charged particles to atoms can still be observed in the form of a constant background radiation that permeates the universe, and can give us information on the structure of the universe at that time.

Measurements of the multipole moments (Figure 1.5) in the CMB yield a prediction for the amount of baryonic and non-baryonic ("normal") matter in the universe $[12]$. The amount of baryonic matter predicted is consistent with observations, while the amount of non-baryonic matter predicted is consistent with the amount of dark matter that is expected to exist in the universe from astronomical observations such as those described above. Taken together with other indirect evidences (such as from Big Bang nucleosynthesis [13] and large scale structure in the universe [14]), a strong case exists for the existence of non-baryonic dark matter.

\section{Proposed Explanations for Dark Matter}

The three most discussed candidates for dark matter are Massive Compact Halo Objects (MaCHOs), Weakly Interacting Massive Particles (WIMPs), and axions. MaCHOs would be made up of normal, baryonic matter that is very cold, and thus invisible to our telescopes [4]. WIMPs are not predicted by the standard model, but many theories, such as super-symmetry [15], that attempt to find a theory more fundamental (or "natural") than the standard model predict them. Axions [16], [17] are

predicted by a popular solution to the Strong CP Problem in the Standard Model, 


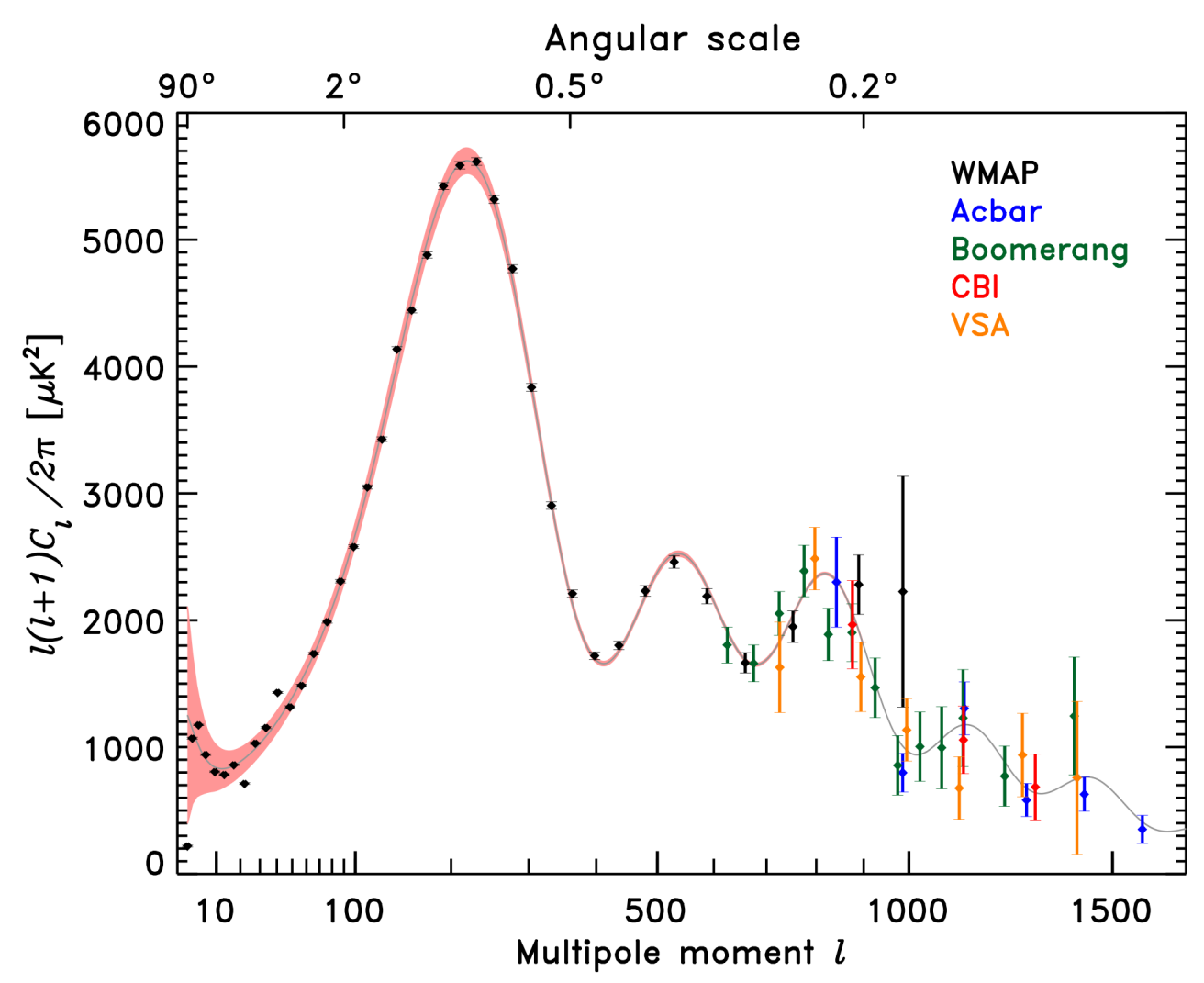

Figure 1.5: CMB multipole moments as measured by the listed experiments and fit with the $\Lambda$ CDM model (see Section 1.3.2) of the Big Bang. This model includes dark matter, with the total amount of dark matter determined by the relative height of the third peak, while the amount of "normal" matter is determined by the relative height of the second peak. (Image credit: NASA) 
but their mass is unknown, so it remains to be seen whether they could be the missing dark matter.

Observations have been made over the last few decades that have made MaCHOs a less likely candidate for dark matter. Gravitational microlensing, a measurement technique that looks for a shift in the light intensity of a star due to the presence of a gravitational field passing in front of it, has been used to search for transits of dark objects, such as black holes, brown and red dwarfs, etc, in front of stars in satellite galaxies to our own and within our galaxy. Searches such as the MACHO Project [18, EROS-2 [19], and observations made by the Hubble Space Telescope [20] have placed an upper limit on the total mass these objects could account for, and the limit is now too low for MaCHOs to account for all the missing dark matter. MaCHOs would also be unable to explain the above mentioned requirement from the CMB that a large amount of non-baryonic matter exists in the universe, providing an additional argument that MaCHOs are no longer a well-motivated dark matter candidate.

Lastly, neutrinos may appear at first glance to be good dark matter candidates, since they only interact through the weak interaction and gravity, making them very difficult to detect. However, the very small mass of neutrinos means that the neutrinos present in the early universe had speeds very close to the speed of light. This prevents them from congregating to form the clouds of dark matter that have been observed throughout the universe, as well as other large scale structures that have existed since shortly after the big bang. In addition, measurements of the CMB have placed a limit

on the total amount of high velocity ("hot") dark matter that restricts neutrinos to making up a very small fraction of the total 21 .

\section{WIMPs}

The term "WIMP" includes any dark matter candidate particle that has a weak coupling to standard model particles, meaning it would interact with normal matter 
through the weak force. Current theories do not necessarily require this coupling to be through the weak force, but they do require that the coupling be quite small [22. The standard cosmological model for the creation of the abundance of WIMPs in the universe is known as the $\Lambda$ CDM (lambda Cold Dark Matter) model 22].

In the $\Lambda$ CDM, WIMPs exist in the very early universe in a state of local thermodynamic equilibrium, where the rate of production is equal to the rate of annihilation $[23]$. However, as the universe expands, its density and temperature decreases, thus reducing the overall rate of interactions. As this expansion takes place, the overall quantity of dark matter particles decreases as particles annihilate faster than they are produced in the lower energy environment. Eventually the rate of dark matter annihilation becomes smaller than the expansion rate of the universe. At this point the quantity of dark matter starts to become fixed. This process is known as thermal freeze out, and we can obtain constraints on the mass and interaction probability of dark matter particles through our understanding of the basics of this process [23].

The equation describing the abundance of dark matter in the modern universe based on the freeze out process just described is [23]:

$$
\Omega_{\chi} h^{2} \approx \frac{T_{0}^{3} x_{f} h^{2}}{\rho_{c} m_{p}\left\langle\sigma_{a} v_{M}\right\rangle_{f}},
$$

where $\Omega_{\chi} h^{2}$ is the relic abundance, $h$ is the reduced Hubble constant $(h=H / 100$, where $H$ is the Hubble constant), $T_{0}=2.35 \times 10^{-13} \mathrm{GeV}$ is the current temperature of the universe, $x_{f}=m_{\chi} / T_{f}, m_{\chi}$ is the WIMP mass, $T_{f}$ is the temperature of the universe at freeze out, $\rho_{c}=3 H^{2} / 8 \pi G$ is the cosmological critical energy density, $m_{p}$ is the reduced Planck mass, $\sigma_{a}$ is the dark matter annihilation cross section, and $v_{M}=\sqrt{\left(\overrightarrow{v_{1}}-\overrightarrow{v_{2}}\right)^{2}-\left(\overrightarrow{v_{1}} \times \overrightarrow{v_{2}}\right)^{2}}$ is the Møller velocity of two annihilating dark matter particles (the relative velocity of the two particles in the centre of mass frame). The angle brackets indicate a thermal average of $\sigma_{a} v_{M}$ at freeze out. 
Assigning values to some of these variables requires some assumptions and approximations, but this can at least give us a sense of the reasonable range of WIMP masses. $\Omega_{\chi} h^{2}$ can be obtained by fitting the CMB multipole moment spectrum as shown in Figure 1.5. The PLANCK data [12] sets this value at $\Omega_{\chi} h^{2}=0.120 \pm 0.001$. As discussed in 23], $x_{f}$ can be approximated by setting the freeze out number density of WIMPs equal to the non-relativistic equilibrium number density. The result of this approximation is:

$$
x_{f}^{3 / 2} e^{-x_{f}} \approx \frac{10^{-8} \mathrm{GeV}}{m_{\chi}} .
$$

From dimensional analysis, a simple assumption can be made about the WIMP cross section:

$$
\sigma_{a} \propto \frac{g^{4}}{m_{\chi}^{2}},
$$

where $g$ is the coupling constant for the annihilation interaction. Combining these approximations gives a range of WIMP masses between approximately $10 \mathrm{MeV}$ and $10 \mathrm{TeV}$. Not all values in this rather large mass range are equally as well motivated as any other. Various models that attempt to extend the standard model of particle physics, such as SuSy, motivate WIMP masses within much narrower ranges.

There has been, and continues to be, great experimental effort put towards the direct detection of WIMPs across a wide range of masses. DEAP-3600 is a part of this experimental effort.

\subsubsection{Strong CP Problem}

\section{C, P, and T Symmetries}

There are several mathematical symmetries within the standard model. These symmetries are mathematical quantities. Some of them are "conserved" throughout all interactions, while others are "broken" by some interactions. Three such symmetries 
are Charge conjugation $(\mathrm{C})$, Parity $(\mathrm{P})$, and Time reversal $(\mathrm{T})[3]$. Charge conjugation symmetry states that an interaction will be identical if all particles involved are swapped with their corresponding anti-particles. Parity symmetry states that a spatial inversion (mirror image) of any interaction is indistinguishable from the original interaction. Time reversal symmetry implies that the interactions are the same if the direction of time is reversed.

C, P, and T-symmetry have all been observed to be conserved in the strong interaction, the electromagnetic interaction (now known to be part of the electroweak interaction), and in gravity [3]. In electromagnetism, for example, a system with a proton and an electron behaves identically as a system with an antiproton and a positron.

However, the weak nuclear interaction (part of the electroweak interaction) is an exception [3]. This is because neither left handed antineutrinos nor right handed neutrinos interact through the weak interaction, while left handed neutrinos and right handed antineutrinos do (handedness here refers to the chirality of the neutrino field). So a weak interaction involving a left handed neutrino can take place, but the analogous interaction involving a left handed antineutrino cannot. This is a violation of both C-symmetry and P-symmetry.

The initial solution to this was to propose a new symmetry known as CP. This basically states that all interactions are identical (invariant) if you swap all particles with their antiparticles and perform a spatial inversion. This symmetry was also shown to be broken, however 24]. On a theoretical level, this is due to the fact that there are three "generations" of particles in the standard model [25]. These generations are differentiated from each other by their "flavor", a property that is important for weak interactions. However, all interactions must take place between particles that are in an quantum-mechanical eigenstate, and there is nothing requiring the flavor eigenstates to commute with the momentum (or mass) eigenstates. So a 
quark or a neutrino in its mass eigenstate in general will simultaneously be in a linear combination of multiple flavor eigenstates. This mixing is described by the quark and neutrino mixing matrices. In the three generation case, a complex phase term appears naturally in the mixing matrix. This phase creates differences between the probabilities of an interaction occurring in particles and in antiparticles, regardless of any spatial inversion.

$\mathrm{CP}$ violating phases appear naturally in both the quark and neutrino mixing matrices, and the CP-violating effects of both phases have been observed experimentally [26]. A CP-violating phase also appears in the Lagrangian describing the strong nuclear interaction 25]:

$$
\mathcal{L}_{Q C D}=\bar{\psi} i \gamma^{\mu} D_{\mu} \psi+m_{q} \bar{\psi} e^{e \theta_{q} \gamma^{5}} \psi+\frac{1}{4} G^{2}+\frac{g_{s}^{2} \theta_{G}}{32 \pi^{2}} G \tilde{G},
$$

where $G$ is the gluon field tensor, $\tilde{G}$ is its dual, $D_{\mu}$ is the gauge covariant derivative, $\gamma^{\mu}$ are the Dirac matrices, $m_{q}$ is the quark mass, $g_{s}$ is the coupling constant of the strong interaction, and $\theta_{q}$ and $\theta_{G}$ are the $\mathrm{CP}$ violating phases. These phases are actually related by:

$$
\bar{\theta}=\theta_{G}-\theta_{q},
$$

which is an invariant phase. This invariant phase is the "observable" quantity within this theory.

To look for evidence of $\mathrm{CP}$ violation in the strong interaction, experimental searches for the existence of a neutron electric dipole moment (nEDM) have been conducted [27]. Since the neutron is a "shapeless" quantum particle, it doesn't really have a "top" or a "bottom". As with any other quantum effect, this isn't a problem with the observational techniques, but a fundamental property of the particles themselves. The neutron does have a property known as "spin", however, which is analogous to angular momentum. Like angular momentum, this spin is defined by 
a vector. This vector could define a kind of "top" for the neutron. But if CP is conserved, it would still be impossible to observe an electric dipole moment in a neutron, because such an observation would violate CPT conservation. To understand this, consider that a time reversal will change the direction of the spin, but not of the EDM. The system is therefore not symmetric under T symmetry. Since CPT conservation is considered a fundamental law of physics, this implies that a violation of CP symmetry is necessary to cancel the violation of $\mathrm{T}$ symmetry in the presence of a neutron EDM.

The small amount of $\mathrm{CP}$ violation known to exist in the weak interaction is expected to produce an extremely small dipole moment in the neutron of approximately $10^{-31} \mathrm{e} \cdot \mathrm{cm}$ 28. Any $\mathrm{CP}$ violation in the strong interaction is expected to have a much larger effect, and is given by 27 :

$$
d_{n} \approx|\bar{\theta}| e m_{q} / M_{n}^{2} \approx|\bar{\theta}| 10^{-16} e \cdot c m
$$

where $m_{q}$ is the quark mass and $M_{n}$ is the neutron mass. However, the current upper limit on the size of the $\mathrm{nEDM}$ is $1.8 \times 10^{-26} e \cdot \mathrm{cm}(90 \%$ C.L.) [29]. This implies that this phase is less than $2.8 \times 10^{-10}$ radians $[30 \mid$. This extremely small (or null) value is considered "unnatural", because this phase is unconstrained by the standard model and the anthropic principle - based on our current understanding of the universe, life would exist even if this phase were non-zero and there would be almost no effect on the overall structure of the universe [31]. It therefore seems reasonable to conclude that something we have yet to understand or discover is forcing the CP-violating phase in the strong interaction to be zero. The fact that this phase seems to be zero independent of the known structure of modern particle physics is known as the "Strong-CP Problem". 


\section{Axions}

The most popular solution to the Strong-CP Problem is given by Peccei-Quinn Theory [16], [32], [33]. Here a global U(1) Peccei-Quinn (PQ) symmetry is introduced, creating a new quantum field which plays the role of the phase term that is naturally present in the QCD Lagrangian. This symmetry is spontaneously broken below a certain interaction energy. In a vacuum, the ground state of the field is thus reduced to the lowest level possible, driving the $\mathrm{CP}$ violating phase term to zero. Due to gluon interactions, the ground state of the field remains greater than zero, and oscillations about the non-zero minimum of this field are manifested as a new particle known as the axion.

Axions could actually be a solution to the dark matter puzzle as well [17]. As the universe expanded and cooled after the Big Bang, the ground state of the axion field slowly decreased. Local concentrations of energy in this field would eventually start to oscillate about the minimum of the potential, as described above. This will occur when the universe has cooled down to the QCD temperature scale, at which point the axion mass becomes large enough and the virtual axion has existed long enough to manifest itself as a particle (an effect related to the Heisenberg uncertainty principle):

$$
m_{a} t_{1} \approx 1
$$

where $m_{a}$ is the axion mass and $t_{1}$ is the critical time at which the oscillation of the axion field has existed long enough for axions to be produced as a real (as opposed to virtual) particle.

Basic axion theory, as proposed by Peccei and Quinn [16], Weinberg [32], and Wilczek [33], does not place any requirements on the energy scale of the spontaneous symmetry breaking. It was proposed [34] that it might be of the same order of magnitude as the electroweak symmetry breaking, but this is not compatible with 
experimental results. More recent axion models have predicted a much higher symmetry breaking scale, which would result in a very light axion mass scale of roughly $1 \mu \mathrm{eV}$, a very low interaction probability, and a very low momentum (in the case of primordial axions), thus making them ideal cold dark matter candidates. The two most popular models are the KSVZ [35], [36] (or hadronic) and DFSZ [37], [38] (or GUT) axions.

In the DFSZ model, a scalar field $(\sigma)$ is added to the Peccei Quinn model and carries a PQ charge. In the KSVZ model, only a superheavy quark (Q) and a scalar field $(\sigma)$ carry a PQ charge (note that in quantum field theory, each global symmetry that is introduced links with quantum fields through "charges"). The experimentally important result in both cases is that $f_{a}=\langle\sigma\rangle>>v_{f}$, where $v_{f}$ is the electroweak symmetry breaking scale (approx. $250 \mathrm{GeV}$ ). In both of these models, the axion mass is given by:

$$
m_{a}=\left(6.3 \times 10^{-6} \frac{10^{12} \mathrm{GeV}}{f_{a}}\right) \mathrm{eV} .
$$

Since the coupling of axions to standard model particles is also proportional to $1 / f_{a}$, this implies that the mass and coupling constants are directly proportional to one another.

These models place no constraints on the axion mass, but they do relate the mass with the axion coupling constant to standard model matter. However, the axion mass in models in which only a single kind of axion exists is constrained by astrophysical and cosmological bounds. Axions can be produced in the core of helium burning stars, white dwarfs, and low mass red giants. These axions would carry away energy. The limit based on models of these stars is $g_{a e}<(2.5-5) \times 10^{-13}$, which implies $f_{a}>(1-2) \times 10^{9} \mathrm{GeV}$ (note that $g_{a e}$ is unit-less). This sets an upper limit on the axion mass: $m_{a}<(3-6) \times 10^{-3} \mathrm{eV}[39 \mid$. In addition, if the symmetry breaking scale is too high, axions will freeze out earlier and will therefore have a greater density. 
A limit on the axion density is set by the measured density of dark matter in the universe. As stated above, the dark matter density is $\Omega_{\chi} h^{2}=0.1186(20)$. The axion density can, therefore, be shown to be:

$$
\Omega_{a} h^{2} \leq 0.5\left[f_{a} / 10^{12} \mathrm{GeV}\right]^{7 / 6} \theta_{i}^{2},
$$

where $\theta_{i}$ is the initial misalignment angle, which is unconstrained. If we assume that $\theta_{i}^{2}$ is of order $\pi$, we get $m_{a}>2.1 \times 10^{-5} \mathrm{eV}$. Masses below this would produce a greater than measured density of dark matter in the universe.

In addition to the above mentioned models, others exist based on SuSy and mirror worlds in which spontaneous symmetry breaking forces the axion mass to be larger while still allowing for low cross sections. In these models, the couplings of axions to standard model particles can be small and compatible with experimental constraints, while the mass of the axion is boosted. It is models like these that are of particular interest for this work, since these allow for much greater axion masses of order $1 \mathrm{MeV}$. As is shown later in this thesis, $5.5 \mathrm{MeV}$ solar axion production is greatly suppressed for sub-eV axion masses.

In one such mirror worlds approach [40], every particle and every interaction has a mirror counterpart. The PQ symmetry of the two particle "worlds" is assumed to be shared in this case. It is possible that the symmetry between the mirror particles and ordinary particles (referred to as "M-parity") could undergo a soft spontaneous symmetry breaking that causes the electroweak scale $\left(v_{F}\right)$ to increase in the mirror sector to some value $v_{F}^{\prime}$. This model assumes the axion follows the original Weinberg-Wilchek (WW) model, in which the axion scale $f_{a}$ is of the same order as the electroweak symmetry breaking scale, and is proportional to it. Since the coupling to matter goes as $1 / f_{a}$, the larger electroweak scale in the mirror world causes the axion to interact less with ordinary matter. Thus, the experimental and observational constraints that 
originally ruled out the WW axion based on its high coupling to matter would allow

this type of axion to exist. In addition, the axion mass goes as $m_{a}^{2} \propto m_{q}^{\prime} \Lambda^{\prime 3} / f_{a}^{2}$ or $m_{a}^{2} \propto \Lambda^{\prime 4} / f_{a}^{2}$, where $m_{q}^{\prime}$ and $\Lambda^{\prime}$ are the lightest mirror quark mass and the mirror QCD scale respectively. The mirror quarks will become more massive than ordinary quarks by $m_{q}^{\prime} \approx\left(v_{F}^{\prime} / v_{F}\right) m_{q}$. This will also cause $\Lambda^{\prime}>\Lambda$. If the increase is strong enough, the axion mass can reach $\mathrm{MeV}$ scale masses even for $f_{a}>10^{4} \mathrm{GeV}$, which is the experimental lower bound.

In a particular SuSy based model [41], the Minimal Supersymmetric Standard Model (MSSM) is modified slightly by the change of a potential term. This leads to a PQ symmetry, but with an additional free mass parameter that gets added to the axion mass. This boost can be as high as the $\mathrm{MeV}$ scale.

Searches for evidence of axions have spanned the last few decades, and are still ongoing. One such search is the Axion Dark Matter eXperiment (ADMX). ADMX consists of a large resonant cavity situated inside a superconducting magnet. The $8 \mathrm{~T}$ magnetic field creates a resonance within the cavity that is tuned to the axion mass that is being searched for. Axions within the galaxy's dark matter halo could transform into a microwave photon under the magnetic field. These photons would then deposit a tiny amount of energy into the cavity. ADMX has ruled out axion masses in the 1.9 to $3.53 \mu \mathrm{eV}$ range for both DFSZ and KSVZ models [42]. In addition, experiments that look for an oscillating neutron EDM have also excluded axion dark matter in the $10^{-24}-10^{-17} \mathrm{eV}$ mass range 43 .

\section{Solar Axions}

We know from observations of astrophysical processes as described above that if axions exist they must have a very weak coupling to standard model matter, meaning that the probability of an axion interacting with normal matter is extremely low. If an axion does interact, there are 3 cross sections of interest to this study (see Figure 2.3 
for the Feynman diagrams). In Compton conversion, the axion scatters off a matter particle, resulting in the axion turning into a photon and an electron being ionized from the atom. In the axio-electric effect the axion is absorbed by an electron that then gets ionized. And lastly, in the inverse Primakov effect the axion is converted into a photon in the presence of an electromagnetic field, such as that within an atom. The Primakov interaction is commonly used to search for evidence of axions, as the axion interaction rate can be controlled by the strength of the electromagnetic field. It is also used to try to convert photons into axions and back into photons again in "light through wall" experiments. Axions are able to do this because they can couple to two photons, allowing them to interact with a virtual photon in an electromagnetic field and change into a photon (or vice versa).

Fundamentally, the axion is an uncharged boson that couples to the electromagnetic interaction. The fundamental axion Feynman diagrams are shown in Figure 1.6 . The axion has an effective coupling to nucleons that allows it to replace the photon in certain interactions. Axions can be produced in the place of photons in an M-type (magnetic type) nuclear transition. As described above, M-type radiation is generated by a varying current or magnetic dipole moment. It was shown in 34 that axions behave as "magnetic photons", meaning that they can replace a photon in Mtype transitions, but not in E-type transitions. This is due to the fact that axions are pseudoscalar particles, so they can only change angular momentum and parity according to the same selection rules that characterize photons produced in M-type transitions.

Stellar fusion involves several different processes. These processes are all interrelated, and are described in the context of sets of fusion reactions or reaction chains. The two known sets that involve the conversion of hydrogen into helium are the CNO cycle and the proton-proton chain. The proton-proton chain dominates in the sun, with the CNO cycle producing only $1.5 \%$ of the total solar luminosity [44]. 

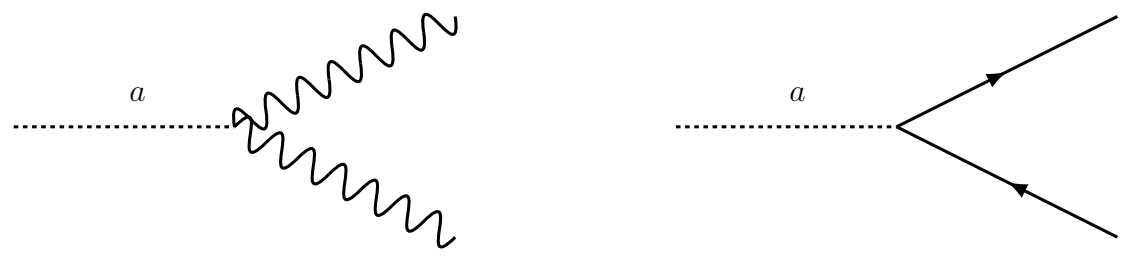

Figure 1.6: Fundamental axion Feynman diagrams

The first reaction in the proton-proton chain [45] is the fusion of two protons into deuterium:

$$
\mathrm{p}+\mathrm{p} \rightarrow \mathrm{d}+\mathrm{e}^{+}+\nu_{e}
$$

The next reaction in the chain is the fusion of the resulting deuterium with another proton to produce ${ }^{3} \mathrm{He}$ :

$$
\mathrm{p}+\mathrm{d} \rightarrow{ }^{3} \mathrm{He}+\gamma(5.5 \mathrm{MeV})
$$

The ${ }^{3} \mathrm{He}$ then fuses into ${ }^{4} \mathrm{He}$ through one of four possible pathways. The most common, occurring $86 \%$ of the time, is:

$$
{ }^{3} \mathrm{He}+{ }^{3} \mathrm{He} \rightarrow{ }^{4} \mathrm{He}+2 \mathrm{p} .
$$

The proton-deuterium fusion interaction in eq. 1.13 is of particular interest for this work due to the $5.49 \mathrm{MeV} \gamma$ that is produced in this reaction. This is because the $\gamma$ is emitted as the result of an M-type nuclear transition, so, if axion's exist, they could occasionally replace the photon in this process. 


\section{Chapter 2}

\subsection{MeV Solar Axion Theory}

The magnitude of the solar axion signal in DEAP-3600 is dependant on the rate of axion production, the probability that an axion will survive the trip to the detector, and the interaction cross section.

\subsection{Rate of 5.5 MeV Axion Production}

Since proton-deuterium fusion occurs relatively quickly once a deuteron becomes available, the rate of $\left(p+d \rightarrow^{3} \mathrm{He}+\gamma(5.5 \mathrm{MeV})\right)$ in the Sun can be determined if the rate of deuterium production is known. According to solar models, $99.7 \%$ of solar deuterium is produced by proton-proton fusion: $p+p \rightarrow d+e^{+}+\nu_{e}$. The $p p$ neutrino flux has been measured to be $6.0 \times 10^{10} \mathrm{~cm}^{-2} \mathrm{~s}^{-1}[46]$.

\subsubsection{Selection Rules}

The fusion reaction involves multiple nuclear transitions, and these follow what are known as "selection rules". Photons have integer angular momentum, and cannot have 0 angular momentum. When a $\gamma$ is emitted as the product of a nuclear transition, the set of possible angular momenta $(L)$ is determined by the initial $\left(J_{i}\right)$ and final 
$\left(J_{f}\right)$ angular momenta of the nucleus:

$$
L=J_{i} \otimes J_{f}
$$

The angular momentum of the emitted photon can therefore have any value between $\left|j_{i}-j_{f}\right|$ and $\left|j_{i}+j_{f}\right|$ except $l=0$. This is one of the "selection rules" that must be followed by the nuclear reaction.

In addition to angular momentum, parity must also be conserved. The value of the photon angular momentum determines whether the transition is a dipole $(l=1)$, quadrupole $(l=2)$, octupole $(l=3)$, etc. Each of these types of transitions has opposite parity from the one before. The photon itself can be generated either by an oscillating charge or by a varying current or magnetic moment. The former is an electric (E) transition, while the latter is a magnetic (M) transition. These also have opposite parity. Transitions are labelled according to the photon's angular momentum and whether the transition was electric or magnetic. For example, an electric quadrupole transition is labelled E2. The parity selection rule resulting from all this is:

$$
\begin{aligned}
\Delta P(E l) & =(-1)^{l} \\
\Delta P(M l) & =(-1)^{l+1},
\end{aligned}
$$

where $P()$ refers to the parity of the photon produced by a transition (which is equal to the difference in parity between the initial and final nuclear states), and $l$ refers to the photon's angular momentum.

\subsubsection{Solar Axion Production Mechanism}

The proton, deuteron, and ${ }^{3} \mathrm{He}$ ground states have positive parity. The ${ }^{3} \mathrm{He}$ ground state has total angular momentum $J=1 / 2$. Since protons and neutrons have spin 
$1 / 2$, the deuteron has spin 0 or 1 :

$$
S_{d}=S_{p} \otimes S_{p}=1 / 2 \otimes 1 / 2=0 \oplus 1
$$

Upon fusing, the combined proton-deuteron state will have total spin $1 / 2$ or $3 / 2$ :

$$
S_{3 e^{*}}=S_{d} \otimes S_{p}=(0 \oplus 1) \otimes 1 / 2=1 / 2 \oplus 1 / 2 \oplus 3 / 2 .
$$

The orbital angular momentum $(l)$ is undetermined. If $l=0$ for the fused system, we can have a photon produced with $l=1 / 2+1 / 2=1, l=3 / 2-1 / 2=1$, or $l=3 / 2+1 / 2=2$. Since there is no parity change, this will result in M1 or E2 radiation. If $l=1$ for the fused system, the total angular momentum can be $j=1+1 / 2=3 / 2, j=1-1 / 2=1 / 2, j=1+3 / 2=5 / 2$, and $j=3 / 2-1=1 / 2$. This leads to possible photon angular momenta of 1,2, and 3, corresponding to M1, E2, and M3. This is summarized below:

$$
\begin{array}{r}
J_{3 e^{*}}=S_{3 e^{*}} \otimes L_{p+d}=(1 / 2 \oplus 1 / 2 \oplus 3 / 2) \otimes(0 \oplus 1 \oplus \ldots)=1 / 2 \oplus 3 / 2 \oplus 5 / 2 \oplus \ldots \\
J_{\gamma}=J_{3_{H} e^{*}} \otimes J_{3} H e(1 / 2 \oplus 3 / 2 \oplus 5 / 2 \oplus \ldots) \otimes 1 / 2=0 \oplus 1 \oplus 2 \oplus 3 \oplus \ldots
\end{array}
$$

The lowest orders dominate, so we only need to consider the M1 and E1 transitions. The axion requires an M-type transition in order to be produced, so the M1 transition is of interest for this work. The fraction of nuclear transitions in this reaction at order $1 \mathrm{keV}$ that produce an M1 $\gamma$ is $\zeta=0.5547$.

We want to know what fraction of M1 transitions in proton-deuterium fusion in the Sun could produce an axion instead of a $\gamma$. This fraction depends on the effective coupling of axions to nucleons, $g_{A N}$. This coupling has an isoscaler $\left(g_{0 A N}\right)$ component and an isovector $\left(g_{3 A N}\right)$ component. However, the M1 transition in $p d$ fusion is 
predominantly an isovector transition, so the $g_{0 A N}$ coupling has no significant effect here. The ratio of axion to $\gamma$ production in $p p$ fusion is given by [48]:

$$
\frac{\omega_{A}}{\omega_{\gamma}}=\frac{\zeta}{2 \pi \alpha}\left[\frac{g_{3 A N}}{\mu_{3}}\right]^{2}\left(\frac{p_{A}}{p_{\gamma}}\right)^{3}=0.54\left(g_{A N}^{3}\right)^{2}\left(\frac{p_{A}}{p_{\gamma}}\right)^{3}
$$

where $\alpha$ is the fine structure constant $(1 / 137), \mu_{3}$ is the isovector magnetic moment, $p_{A}$ is the momentum of the $5.5 \mathrm{MeV}$ (kinetic energy plus mass) axion, and $p_{\gamma}$ is the $5.5 \mathrm{MeV} \gamma$ momentum.

The coupling constant is proportional to the axion mass (this isn't necessarily true for all axion-like particles (ALPs)). In the hadronic case, this dependence can be expressed as 48 :

$$
g_{3 A N}=-2.75 \times 10^{-8} m_{A},
$$

where the axion mass, $m_{A}$, is expressed in $\mathrm{eV}$.

In the GUT (Grand-Unified Theory) axion model, the corresponding relation depends on an unconstrained parameter, so an equation like 2.7 can't be written as easily.

Equation 2.6 is plotted in Figures 2.1 and 2.2 for hadronic and GUT axion models respectively.

The axion flux at earth can therefore be calculated from the flux of pp solar neutrinos. The pp solar neutrino flux at earth is: $\Phi_{\nu p p}=6.0 \times 10^{10} \mathrm{~cm}^{-2} \mathrm{~s}^{-1}[46]$. So the axion flux before accounting for axion decays in transit is:

$$
\Phi_{A 0}=\Phi_{\nu p p} \frac{\omega_{A}}{\omega_{\gamma}}=3.23 \times 10^{10}\left(g_{3 A N}\right)^{2}\left(\frac{p_{A}}{p_{\gamma}}\right)^{3},
$$

where $\Phi_{A 0}$ is in units of $\mathrm{cm}^{-2} \mathrm{~s}^{-1}$. 


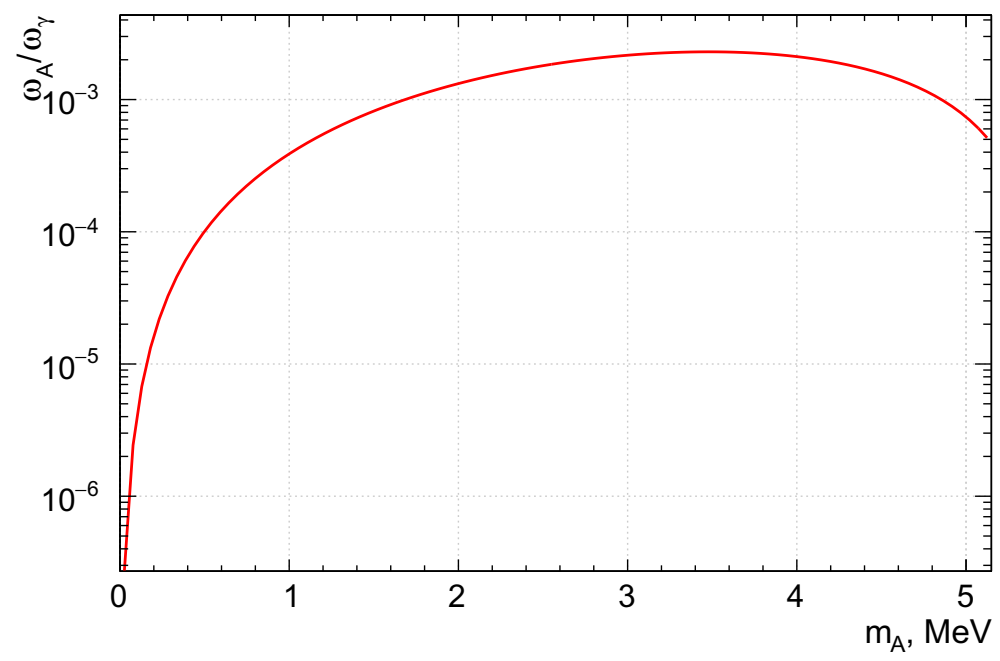

Figure 2.1: $\omega_{A} / \omega_{\gamma}$ for hadronic axions.

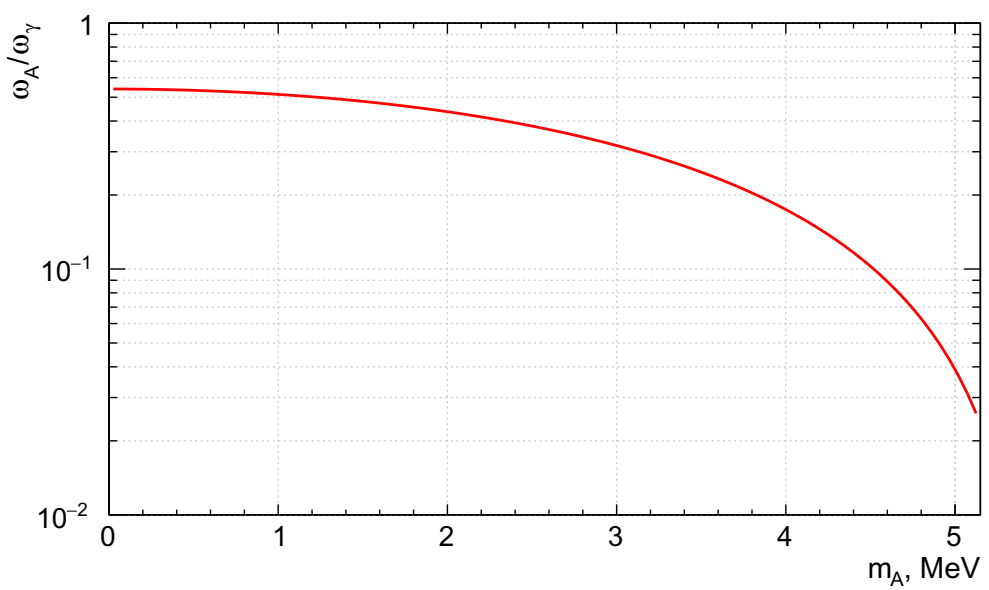

Figure 2.2: $\omega_{A} / \omega_{\gamma}$ for GUT axions. Here $g_{A N}^{3}$ was set to 1 . 
In the hadronic axion model we can combine eq. 2.8 with 2.7 to get:

$$
\Phi_{A 0}=2.44 \times 10^{-5} m_{A}^{2}\left(\frac{p_{A}}{p_{\gamma}}\right)^{3},
$$

where $m_{A}$ is in $\mathrm{eV}$. The effect of axion decay in transit to earth will be addressed at the end of the next section.

\subsection{Axion Interaction Cross Sections}

The probability of an interaction given an axion in the detector is given by the interaction cross section. The Feynman diagrams for the five cross sections of interest for this work are shown in Figure 2.3. The vertices in each diagram represent an interaction between particles, and together the vertices form the overall interaction represented by the diagram itself. Each vertex involves a coupling constant, which determines the probability that the interaction represented by a given vertex occurs. The combined probability that all interaction vertices combine to form the overall interaction represented by the diagram is given by the interaction "cross-section".

The coupling constants all depend on the axion decay constant $f_{A}$ [16], which is one of the most important free parameters in the axion theory. $f_{A}$ is related to the axion mass by 48 :

$$
f_{A}=\frac{6.0 \times 10^{15}}{m_{A}}
$$

with $m_{A}$ and $f_{A}$ in $\mathrm{eV}$.

The axion-photon-photon coupling constant is [49]:

$$
g_{A \gamma}=\frac{\alpha C_{A \gamma \gamma}}{2 \pi f_{A}}=1.9 \times 10^{-19} C_{A \gamma \gamma} m_{A}
$$

where $C_{A \gamma \gamma}$ is a model-dependant constant. $C_{A \gamma \gamma}=0.74$ for GUT axions, and $C_{A \gamma \gamma}=$ 


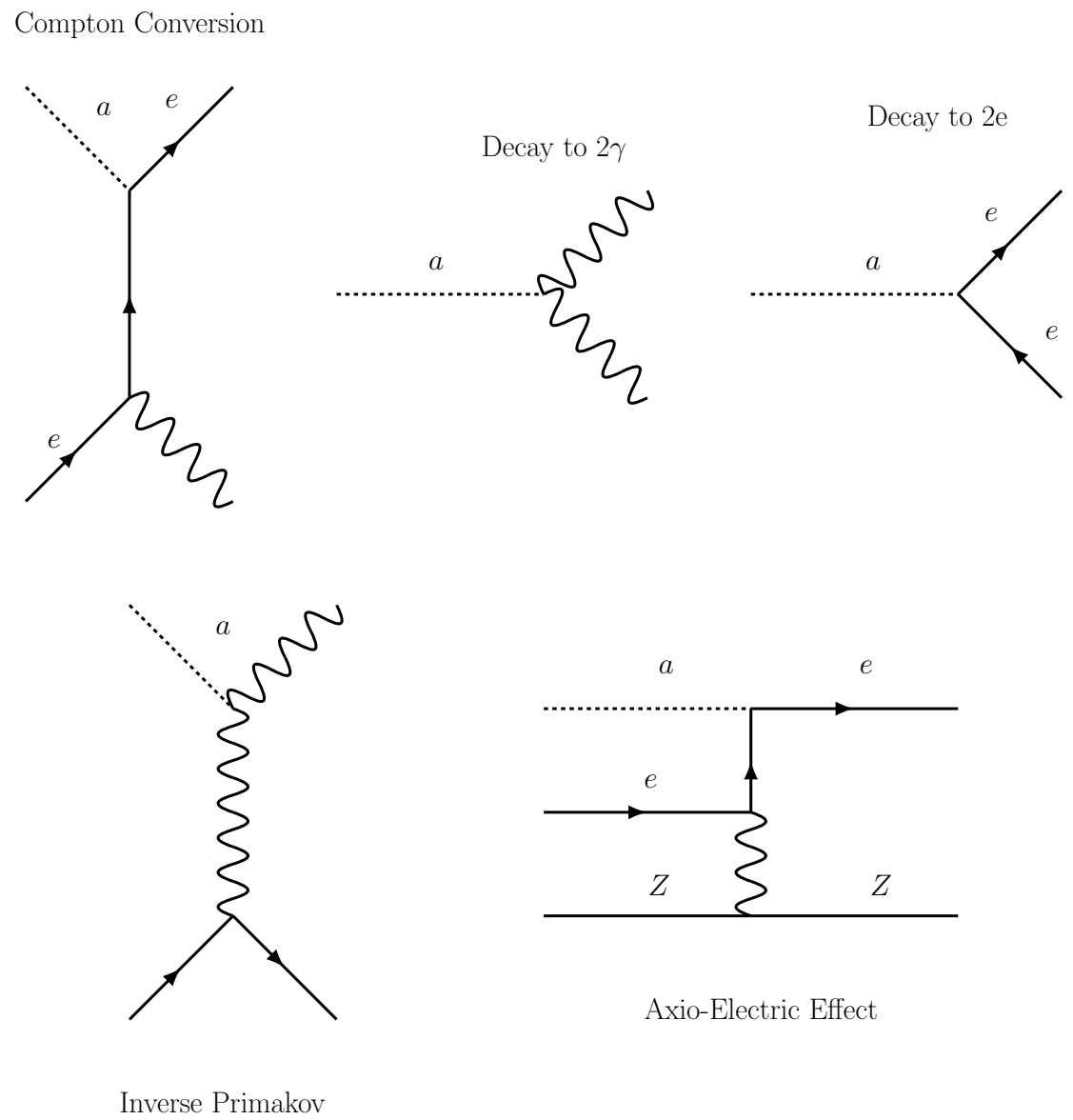

Figure 2.3: Axion cross sections of interest for this work. Time is on the horizontal axis. a represents the axion, $e$ represents either an electron or positron, and $Z$ represents an atom. Solid lines without a label can be a quark or electron/positron. 


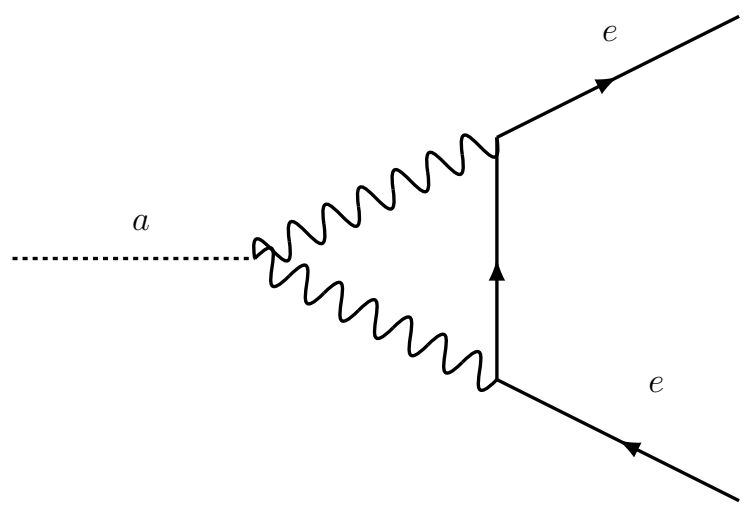

Figure 2.4: 1 loop order coupling of axion to electron. In the hadronic axion model, this is the dominant term contributing to the axion-electron interaction vertex.

-1.92 for hadronic axions.

The axion-electron-electron coupling constant in the GUT axion model is [49]:

$$
g_{A e}=\frac{C_{e} m_{e}}{f_{A}}=1.7 \times 10^{-16} C_{e} m_{e} m_{A}
$$

where $m_{e}$ is the electron mass and $C_{e}=1 / 3 \cos ^{2}\left(\beta_{D F S Z}\right) . \quad \beta_{D F S Z}$ is an arbitrary angle. The hadronic axion model does not have an axion-electron coupling constant. However, it is still possible for the axion in this model to interact with electrons through a loop, as shown in Figure 2.4. This loop would replace the axion-electron vertices in Figure 2.3 .

We can now look at the probability of an interaction in Figure 2.3 occurring. The cross section for Compton Conversion is 49 :

$$
\begin{gathered}
\sigma_{C C}=\frac{g_{A e}^{2} \alpha}{8 m_{e}^{2} p_{A}}\left[\frac{2 m_{e}^{2}\left(m_{e}+E_{A}\right) y}{\left(m_{e}^{2}+y\right)^{2}}+\frac{4 m_{e}\left(m_{A}^{4}+2 m_{A}^{2} m_{e}^{2}-4 m_{e}^{2} E_{A}^{2}\right)}{y\left(m_{e}^{2}+y\right)}\right. \\
\left.+\frac{4 m_{e}^{2} p_{A}^{2}+m_{A}^{4}}{p_{A} y} \ln \left(\frac{m_{e}+E_{A}+p_{A}}{m_{e}+E_{A}-p_{A}}\right)\right],
\end{gathered}
$$

where $y=2 m E_{A}+m_{A}^{2}$. The signal in the detector is one electron and one photon, 


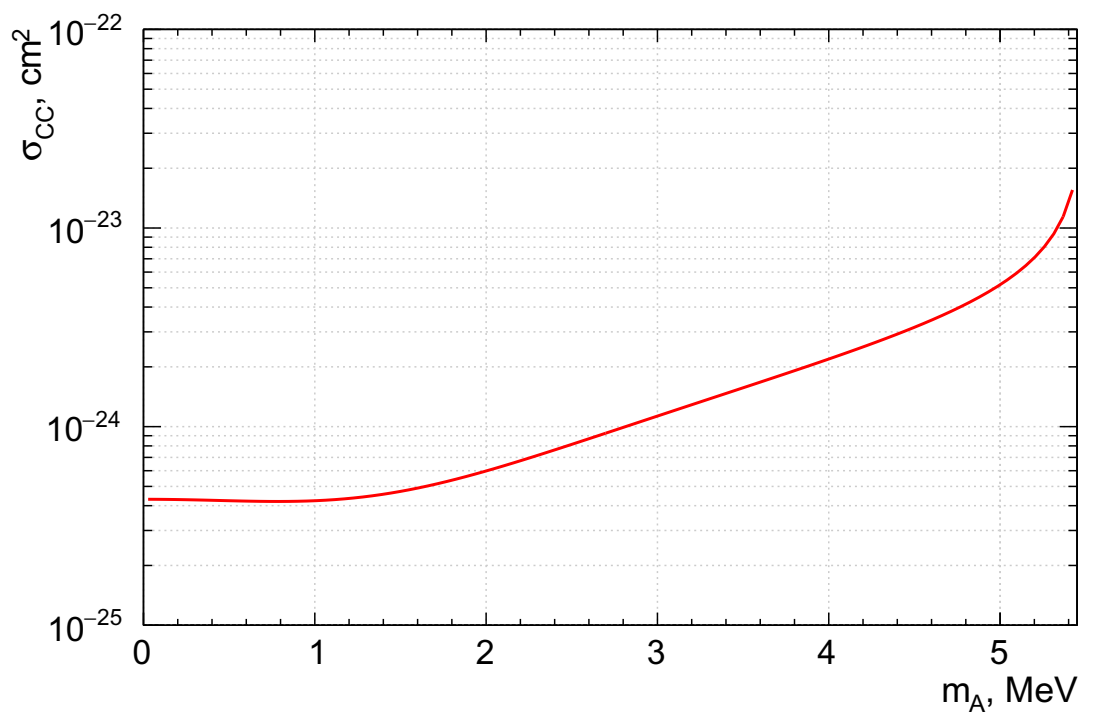

Figure 2.5: The axion Compton conversion cross section (see eq. 2.13) for DEAP3600 , with $g_{A} e=1$.

with a combined kinetic energy of $5.5 \mathrm{MeV}$. The cross section is plotted for DEAP3600 in Figure 2.5 for $g_{A} e=1$. The energy distributions of the electron and $\gamma$ are determined by the cross section and are shown in Figure 2.6.

The inverse Primakov cross section is [49]:

$$
\sigma_{P C}=g_{A \gamma}^{2} \frac{Z^{2} \alpha}{2}\left[\frac{1+\beta^{2}}{2 \beta^{2}} \ln \left(\frac{1+\beta}{1-\beta}\right)-\frac{1}{\beta}\right]
$$

where $Z$ is the target's atomic number and $\beta=p_{A} / E_{A}$. The signal in the detector is one 5.5 MeV photon. The cross section is plotted for DEAP-3600 in Figure 2.7 for $g_{A \gamma}=1$.

The axio-electric effect is the axion analog of the photo-electric effect. The requirement is that $E_{A}>E_{b}$, where $E_{b}$ is the binding energy of the electron to the 

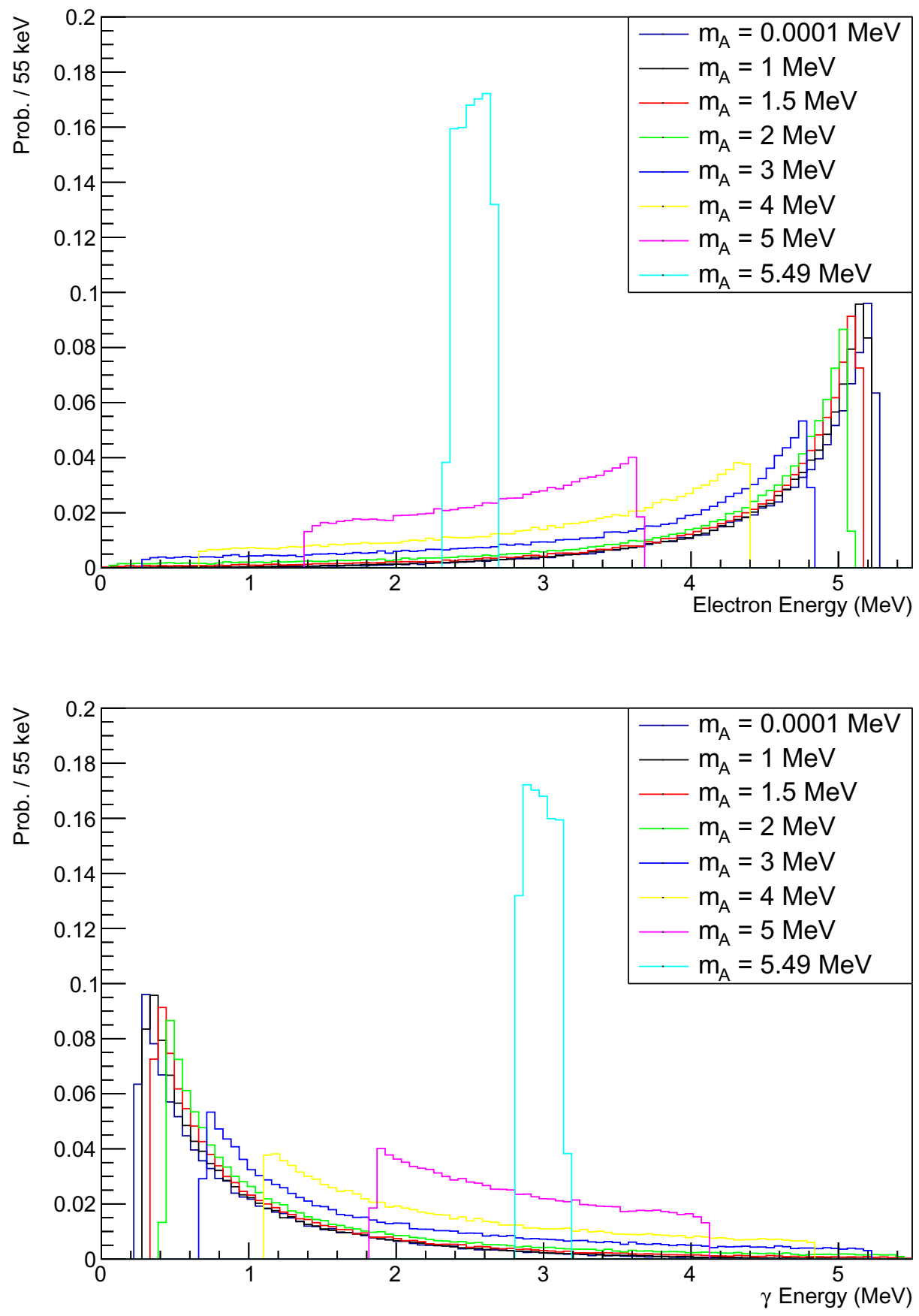

Figure 2.6: The energy distribution of the electron (top) and the $\gamma$ (bottom) produced by a Compton conversion of an axion into a photon for different axion masses. These plots were created as part of the validation of the event generator written for the analysis described later in this thesis. 


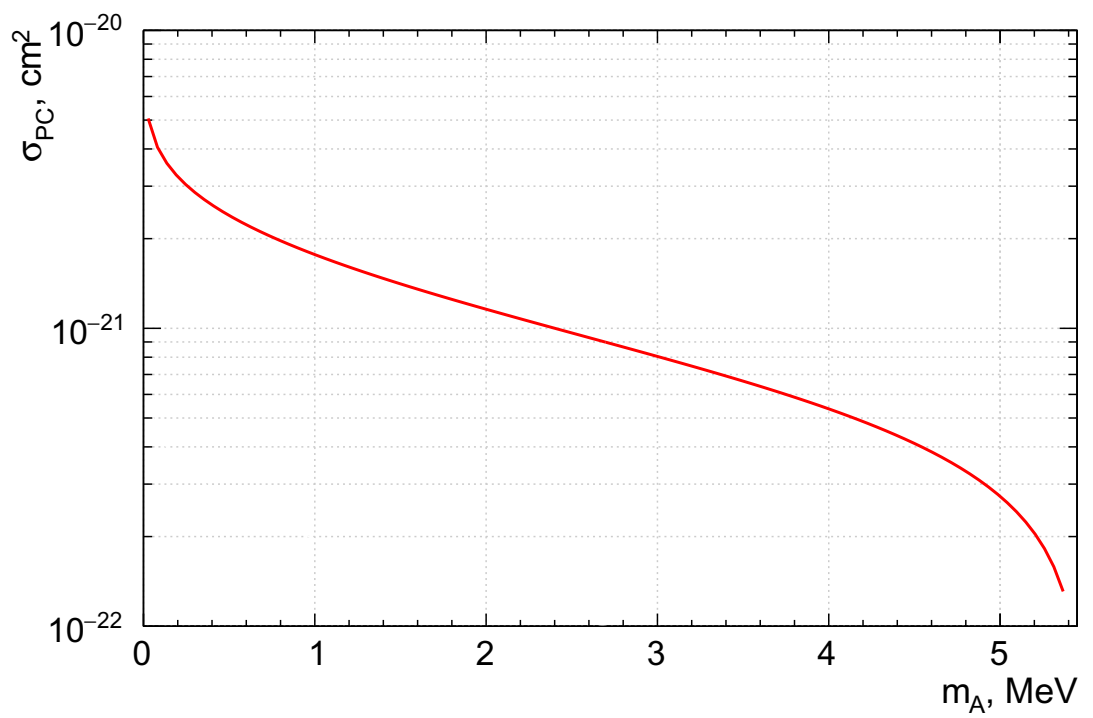

Figure 2.7: The axion inverse Primakov conversion cross section (see eq. 2.14) for DEAP-3600 with $g_{A \gamma}=1 \mathrm{MeV}^{-1}$.

nucleus. The cross section of the axio-electric effect (for $E_{A}>>E_{b}$ ) is 48 :

$$
\begin{aligned}
\sigma_{A e}=2\left(Z \alpha m_{e}\right)^{5} \frac{g_{A e}^{2} p_{e}}{m_{e}^{2} p_{A}}[ & \frac{4 E_{A}\left(E_{A}^{2}+m_{A}^{2}\right)}{\left(p_{A}^{2}-p_{e}^{2}\right)^{4}}-\frac{2 E_{A}}{\left(p_{A}^{2}-p_{e}^{2}\right)^{3}}-\frac{64 p_{e}^{2} p_{A}^{2} m_{e} m_{A}^{2}}{3\left(p_{A}^{2}-p_{e}^{2}\right)^{6}} \\
& \left.-\frac{16 m_{A}^{2} p_{A}^{2}\left(E_{e}-m_{e}\right)}{\left(p_{A}^{2}-p_{e}^{2}\right)^{5}}-\frac{E_{A}}{p_{e} p_{A}\left(p_{A}^{2}-p_{e}^{2}\right)^{2}} \ln \left(\frac{p_{e}+p_{A}}{p_{e}-p_{A}}\right)\right],
\end{aligned}
$$

where $p_{e}$ is the momentum of the recoiling electron and $E_{e}$ is the total energy of the recoiling electron. The signal in the detector is one electron with $5.5 \mathrm{MeV}$ kinetic energy. The cross section is plotted in Figure 2.8 for DEAP-3600 for $g_{A} e=1$.

The mean decay time for the two photon channel is [49:

$$
\tau_{2 \gamma}=\frac{64 \pi}{g_{A \gamma}^{2} m_{A}^{3}} .
$$




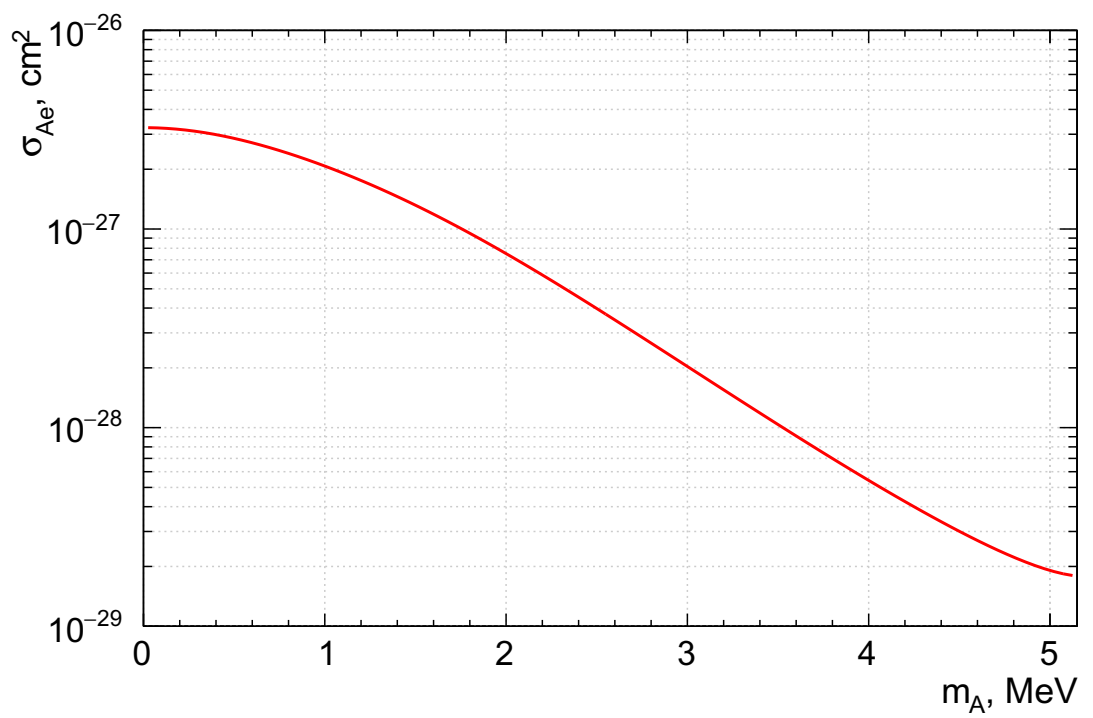

Figure 2.8: The axio-electric effect cross section in DEAP-3600 for $g_{A} e=1$.

Combining eq. 2.16 with eqs. 2.11 and 2.10 gives:

$$
\tau_{2 \gamma}=3.5 \times 10^{24} m_{A}^{-5} C_{A \gamma \gamma}^{-2},
$$

where $m_{A}$ is in $\mathrm{eV}$ and $\tau_{2 \gamma}$ is in seconds.

\subsubsection{Axion Decays in Transit from the Sun}

In order to take into account the decay of axions in transit from the Sun, we need the time of flight. This is given by [49]:

$$
\tau_{f}=\frac{m_{A} L}{p_{A} c}
$$

where $L$ is the distance between earth and the Sun and $c$ is the speed of light.

The flux of axions at Earth after accounting for axion decays into the two photon 
channel can now be calculated. It is given by:

$$
\Phi_{A}=\Phi_{A 0} e^{-\tau_{f} / \tau_{2 \gamma}}
$$

All axion and ALP models are effected by this decay, since they all include a coupling to 2 photons. The strength of this coupling varies among models, however.

The last diagram in Figure 2.3 we have left to consider is the decay of the axion into an electron/positron pair. This decay is only relevant when $m_{A}>2 m_{e}$. The mean decay time of an axion into an electron/positron pair is:

$$
\tau_{e^{+} e^{-}}=\frac{8 \pi}{g_{A e}^{2} \sqrt{m_{A}^{2}-4 m_{e}^{2}}} .
$$

Ignoring the 2 photon decay, the axion flux at the earth for axions with $m_{A}>2 m_{e}$ is:

$$
\Phi_{A e^{+} e^{-}}=\Phi_{A 0} e^{-\tau_{f} / \tau_{e^{+} e^{-}}}
$$

\subsection{Axion Escape from the Sun}

We should also account for the escape of axions from the Sun. Eq. 2.6 allows us to determine the number of axions produced in the Sun, but doesn't take account for any change in axion flux as it makes its way out of the Sun. Compton conversion, Primakov conversion, and the axio-electric effect are all capable of reducing the axion flux if enough matter is present. This was examined in detail by [50].

There are approximately $N_{e_{\text {sun }}}=7 \times 10^{35}$ electrons per $\mathrm{cm}^{2}$ between the centre of the Sun and its surface. As can be seen in Figure 2.5, for masses below $1 \mathrm{MeV}$, the Compton conversion cross section is approximately constant at $\sigma_{C C}=g_{A e}^{2} 4 \times$ $10^{-25} \mathrm{~cm}^{2}$. Applying the condition that $\sigma_{C C} N_{e_{\text {sun }}}<0.1$ gives $g_{A e}<6 \times 10^{-7}$.

For the effect of Primakov conversion on hydrogen we just need to scale down 


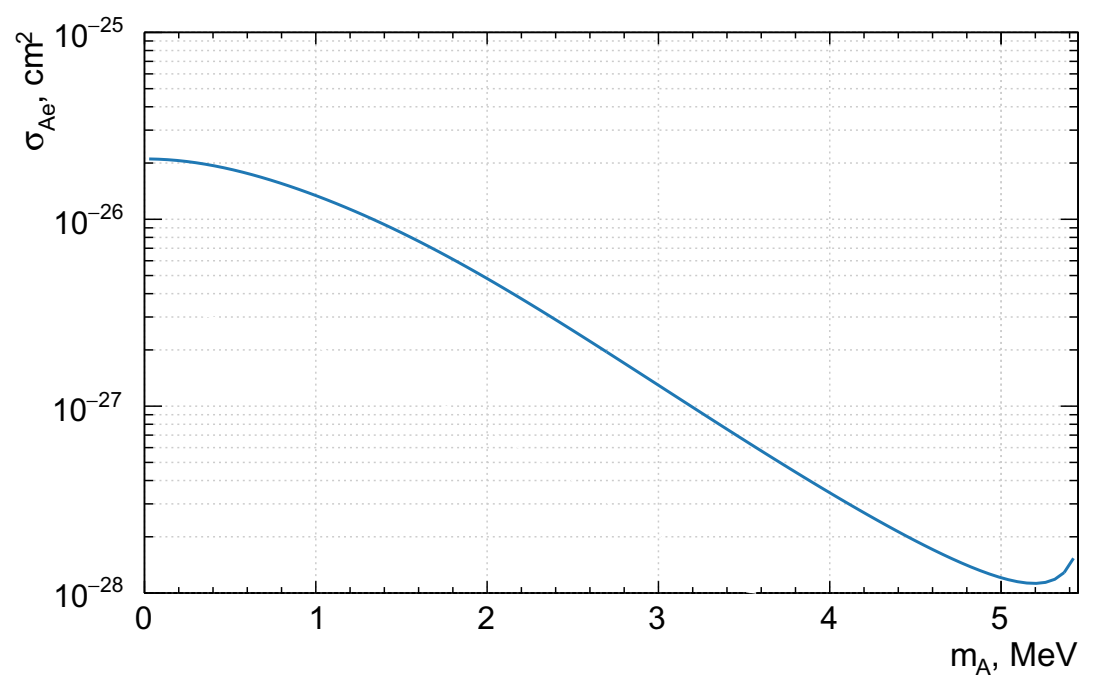

Figure 2.9: Axio-electric effect cross section for iron with $g_{A e}=1$ for $5.5 \mathrm{MeV}$ axions.

the cross section in Figure 2.7 by a factor of $18^{2}$. This gives a maximum cross section of approximately $\sigma_{\gamma \gamma}=g_{A \gamma}^{2} 2 \times 10^{-23} \mathrm{~cm}^{2}$. Given that there are approximately $N_{p_{\text {sun }}}=5 \times 10^{35}$ protons in the Sun, and applying the condition that $\sigma_{P C} N_{p_{\text {sun }}}<0.1$, gives $g_{A \gamma}<1 \times 10^{-7} \mathrm{MeV}^{-1}$. Other elements in the Sun do not have a significant impact due to their low concentrations.

The axio-electric effect scales as $Z^{5}$, so we need to consider elements heavier than hydrogen. As shown in [50], iron is responsible for the strongest constraint due to the relative abundances of elements in the Sun. As seen in Figure 2.9, the cross section has its maximum at $2 \times 10^{-26} \mathrm{~cm}^{2}$. The ratio of iron to hydrogen in the Sun is $N_{F e_{\text {sun }}} / N_{H_{\text {sun }}}=2.8 \times 10^{-5}$. Applying our constraint of $\sigma_{A e} N_{p_{\text {sun }}} N_{F e_{\text {sun }}} / N_{H_{\text {sun }}}<0.1$, we get $g_{A e}<5 \times 10^{-4}$.

These three constraints put an upper limit on the sensitivity of this type of axion search. They are summarized in table 2.1. 


\begin{tabular}{c|c} 
Interaction & Constraint \\
\hline Compton & $g_{A e}<6 \times 10^{-7}$ \\
Primakov & $g_{A \gamma}<1 \times 10^{-7} \mathrm{MeV}^{-1}$ \\
Axio-electric & $g_{A e}<5 \times 10^{-} 4$
\end{tabular}

Table 2.1: Summary of constraints that will allow at least $90 \%$ of axions to escape the Sun for the relevant interactions with solar matter. If the true axion coupling is outside these constraints, experimental searches for axions produced in the core of the Sun will be unable to detect them.

\subsection{Rates of Interactions in the Detector}

If we know the detector volume $\mathrm{V}$, we can now calculate the number of axions that would decay into two photons within the detector. The number of decays per unit time is given by:

$$
N_{\gamma}=\Phi_{A} \frac{V m_{A}}{c p_{A} \tau_{2 \gamma}} .
$$

$N_{\gamma}$ is plotted in Figure 2.10 for DEAP-3600 for the hadronic axion model.

We can make the same plot for the rate of Primakov conversions in the hadronic model, since the Primakov conversion cross section depends on the $g_{A \gamma}$ coupling constant. However, this also means that any limits on this coupling constant based on the observed rate of Primakov conversions in our detector will also need to take into account the reduction in flux due to the decay of axions in transit from the Sun. This rate is given by [49]:

$$
N_{P C}=\sigma \cdot \chi \cdot N_{Z} \cdot \Phi_{A}
$$

where $N_{Z}$ is the number of atoms with $Z$ protons in the detector and $\chi$ corrects for the screening of nuclear charge by the atomic electrons. It is [51]:

$$
\chi=\frac{2 \ln \left(2 E_{A} / m_{A}\right)-1+e^{-x}\left(1-e^{-x} / 2\right)+(x+1 / 2) E_{1}(2 x)-(1+x) E_{1}(x)}{2 \ln \left(2 E_{A} / m_{A}\right)-1},
$$




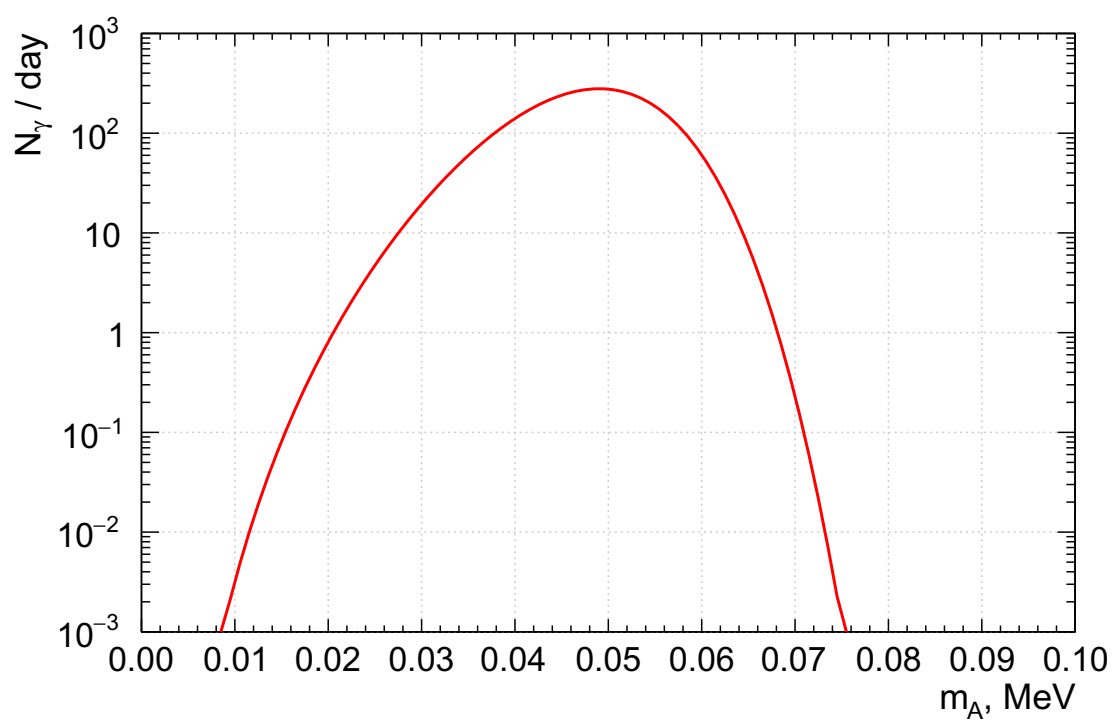

Figure 2.10: The number of axion decays to two photons expected within the DEAP-3600 detector in one day for the hadronic axion model. The drop in rate as mass increases is due to the increased probability of decay, which reduces the axion flux at the detector.

where $x=\left(r_{0} m_{A}^{2} / 4 E_{A}\right)^{2}, r_{0}$ is the atomic radius, and $E_{1}(x)=\int_{x}^{\infty}\left(e^{-t} / t\right) d t$ (the exponential integral). The resulting daily rate of Primakov conversions of hadronic axions on argon in DEAP-3600 is plotted in Figure 2.11.

The number of axions that would decay through the $\mathrm{e}^{+} \mathrm{e}^{-}$channel in a detector of volume $\mathrm{V}$ is:

$$
N_{e^{+} e^{-}}=\Phi_{A e^{+} e^{-}} \frac{V m_{A}}{c p_{A} \tau_{e^{+} e^{-}}} .
$$

However, it was shown in [50] that the decay of $5.5 \mathrm{MeV}$ axions into two photons imposes an upper limit on $g_{A e}$ of $10^{-17}$ to $10^{-15}$ for axion masses between $1.022 \mathrm{MeV}$ and $5.5 \mathrm{MeV}$. This effectively reduces the probability of seeing an axion decay into an electron/positron pair to zero. 


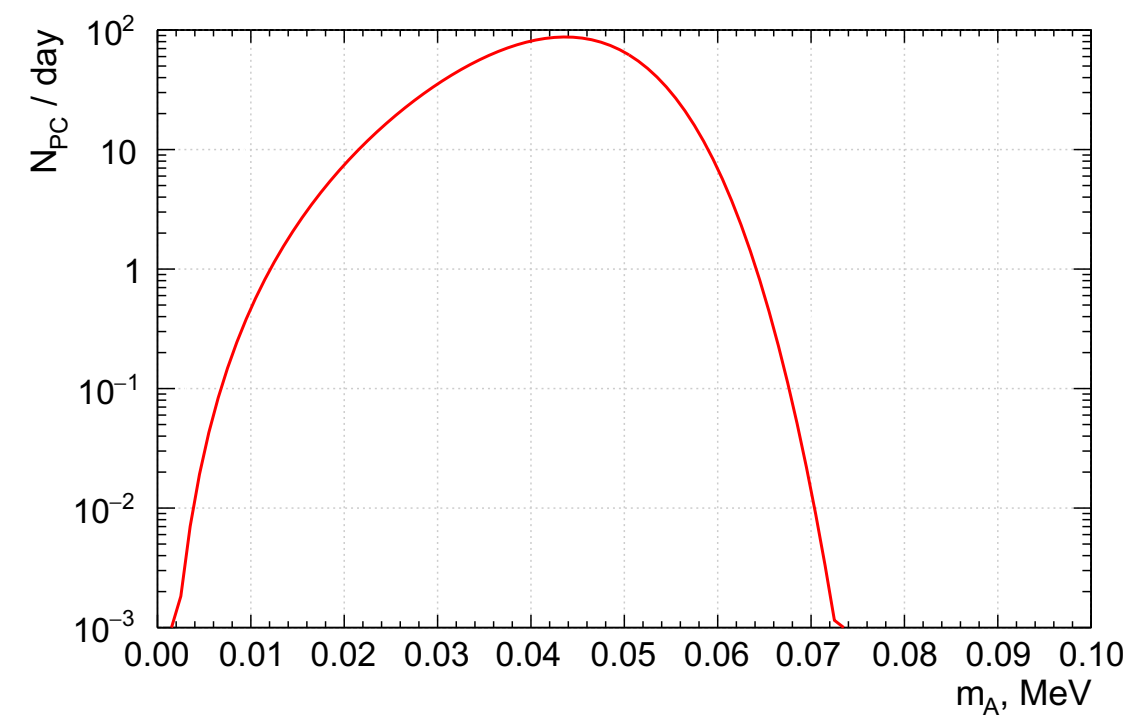

Figure 2.11: Number of Primakov conversions on argon in DEAP-3600 per day for the hadronic axion model. 


\section{Chapter 3}

\section{The DEAP-3600 Experiment}

DEAP-3600 is a dark matter search experiment located $2 \mathrm{~km}$ underground in SNOLAB, Sudbury, Ontario. It is run by the international DEAP Collaboration.

DEAP-3600 is specifically designed to search for $\sim 30 \mathrm{GeV}$ to $\sim 10 \mathrm{TeV}$ mass WIMPs. The low probability of an interaction between a WIMP and "normal" matter lets WIMPs pass through large amounts of matter without interacting, making underground laboratories ideal for direct dark matter experiments like DEAP-3600. It is also important to note that the cross section for interactions with the nucleus is expected to be much larger than that for interactions with electrons, giving an advantage to experiments that can discriminate between the two.

DEAP-3600 is located over $2 \mathrm{~km}$ underground to prevent backgrounds from cosmogenically activated isotopes, solar radiation, and atmospheric shower particles from interacting with the detector. Without the shield provided by the $2 \mathrm{~km}$ of rock, the

detector would be saturated with background events. But WIMPs would be able to pass through freely due to their low interaction cross section. 


\subsection{Detector Design}

The detector design and construction is detailed in [2]. The detector itself is composed of an acrylic sphere filled with liquid argon (LAr) and surrounded by 255 photomultiplier tubes (PMTs) (Figure 3.1). When a particle interacts with the LAr, it produces ultra-violet (UV) scintillation photons with a wavelength of $128 \mathrm{~nm}$. The number of photons produced is proportional to the total energy deposited in the LAr. These photons make their way to the LAr/acrylic interface. Since acrylic is opaque to UV light, a $3 \mu \mathrm{m}$ thick layer of 1,1,4,4-tetraphenyl-1,3-butadiene (TPB) wavelength shifter was deposited on the inner surface of the acrylic volume. When the UV photons reach this layer, they get shifted to visible wavelengths, which allows them to pass through the acrylic light-guides to the PMTs located at the ends of them. Once a photon reaches a PMT, it triggers an avalanche of electrons, which registers as a voltage spike in the readout electronics.

For the purpose of specifying the locations of events within the detector, a coordinate system was defined. The origin of this coordinate system is the centre of the sphere containing the liquid argon. The Z-axis runs vertically toward the neck.

The acrylic sphere has an inner radius of $85 \mathrm{~cm}$. The liquid argon is filled up to $55 \mathrm{~cm}$ above the centre of the acrylic sphere, with the top $30 \mathrm{~cm}$ being filled with gaseous argon. The total liquid argon mass is $3279 \pm 96 \mathrm{~kg}$ [1].

The space between the light-guides is filled with filler blocks made from alternating layers of high density polyethylene and Styrofoam, providing thermal insulation and neutron shielding. The space between the PMTs is filled with Styrofoam.

The main detector volume is accessed through a neck at the top of the sphere. A stainless steel cooling coil is present in the neck to keep the argon temperature between $84 \mathrm{~K}$ and $87 \mathrm{~K}$. Acrylic flow guides at the bottom of the neck encourage the upward flow of warmer gaseous argon to the cooling coils, while also blocking photons 


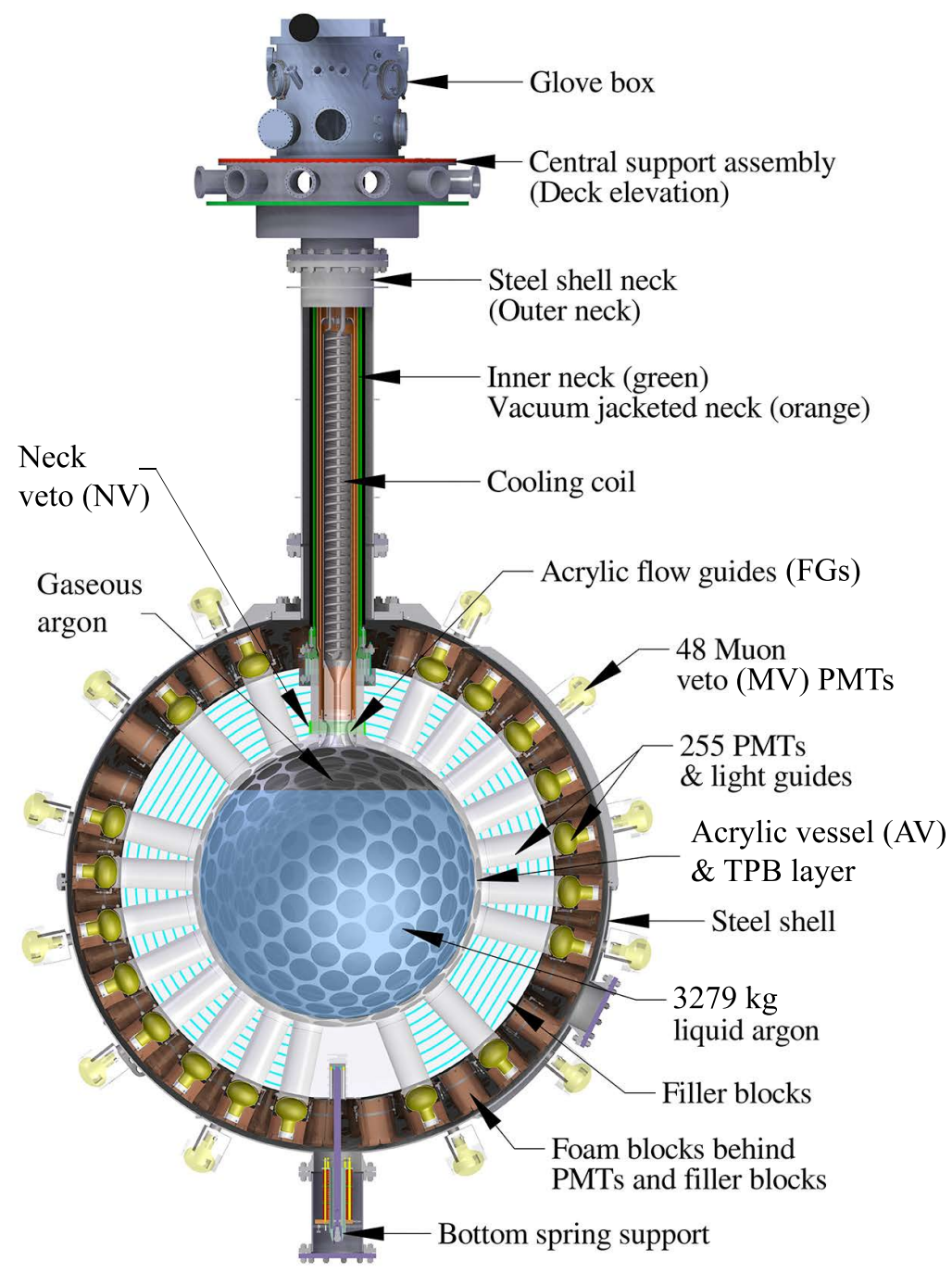

Figure 3.1: Cross-section of the DEAP-3600 detector, with select components highlighted. Note that the liquid argon is filled to $551 \mathrm{~mm}$ above the equator of the acrylic sphere. 
generated by radioactive decays in the neck from reaching the liquid argon volume. The neck is insulated by a vacuum jacket.

The entire detector is covered by a steel shell. A water tank surrounds the steel shell, and acts to shield the detector from external neutrons. The water tank is also an essential component of the muon veto system.

48 outward facing PMTs were installed on the steel shell to collect light produced by muons as they pass through the water tank. These PMTs operate in a self-trigger mode. The 48 PMTs are divided into six groups of eight. When one PMT within a group is above a threshold, that group is labelled as "active". When three groups are active, the veto system sends a signal to the digital trigger module (dtm) to let it know that the trigger condition has been met and the PMTs are read out. When this occurs, all detector events within an amount time after the trigger can be vetoed, making it very easy to later exclude them from the dataset. For the WIMP search, the muon veto time cut is tuned to flag $95 \%$ of muons that enter the water tank.

The residual muon flux from atmospheric showers induced by cosmic rays create a background in the datasets. These showers generate a slew of various particles, most of which are quickly stopped when they reach the surface of the earth. Muons, however, are capable of travelling a significant distance in matter before they are stopped due to their high energy and mass. The muon flux has been measured to be less than 0.27 muons $/ \mathrm{m}^{2} /$ day at SNOLAB, which is $2 \mathrm{~km}$ below the surface. These muons can react with the rock underground to produce neutrons. Both these muon induced neutrons and the muons themselves can enter the detector. If a muon enters, it has a high chance of being vetoed. However, the muon could activate detector components as well as the LAr itself, creating a muon-correlated delayed background. The exclusion of these backgrounds is the motivation for vetoing events up to $1 \mathrm{~s}$ after the muon veto system is triggered. Note that it is still possible for a muonactivated neutrons to enter the detector without the responsible muon entering the 
water tank. However, as has been shown by a collaborator, the probability of the neutron reaching the LAr is quite low. These neutrons are factored into the overall WIMP search background estimate.

\subsection{Signal and Signal Detection}

Interactions in DEAP-3600 are detected based on the light they produce. The primary means of light production is scintillation of the liquid argon target. Though the microphysics is not well understood, I will explain a simple model [52] that is often used to understand, at least qualitatively, the scintillation process in argon.

When a particle of sufficient energy enters the liquid argon, it causes the ionization of some of the argon atoms. During this process, some of the argon atoms will receive some energy, but not enough to be ionized. Argon excitons are produced when a valence electron is promoted to the first excited state. This electron can bond with the ground state valence electron of another argon atom to form an $\mathrm{Ar}_{2}$ dimer. This dimer later decays back into 2 ground state argon atoms with the emission of a $128 \mathrm{~nm}$ photon. This process is summarized in eq. 3.1 .

$$
\begin{aligned}
\mathrm{Ar}+\mathrm{Ar}^{*} & \rightarrow \mathrm{Ar}_{2}^{*} \\
\mathrm{Ar}_{2}^{*} & \rightarrow 2 \mathrm{Ar}+\gamma
\end{aligned}
$$

Dimers can also form when an ionized argon atom and a ground state argon atom form a bond by sharing the valence electron of the ground state atom. This bond eventually breaks when an electron recombines with the ion. Upon recombination, the former ion enters a doubly excited state, which decays non-radiatively to a single excited state. From there it can form another bond with a ground state argon atom as described in eq. 3.1 to form a new dimer. This entire process is summarized in 
eq. 3.2 .

$$
\begin{aligned}
\mathrm{Ar}^{+}+\mathrm{Ar} & \rightarrow \mathrm{Ar}_{2}^{+} \\
\mathrm{Ar}_{2}^{+}+\mathrm{e}^{-} & \rightarrow \mathrm{Ar}+\mathrm{Ar}^{* *} \\
\mathrm{Ar}^{* *}+\mathrm{Ar} & \rightarrow \mathrm{Ar}^{*}+\mathrm{Ar}+\text { heat } \\
\mathrm{Ar}+\mathrm{Ar}^{*} & \rightarrow \mathrm{Ar}_{2}^{*} \\
\mathrm{Ar}_{2}^{*} & \rightarrow 2 \mathrm{Ar}+\gamma
\end{aligned}
$$

The singly excited dimer state in eq. 3.1 and eq. 3.2 can be thought of as an electron orbiting a $\mathrm{Ar}_{2}^{+}$core. The electron and the core both have spin-1/2. These spins can add in one of four ways:

$$
\begin{aligned}
|\uparrow \uparrow\rangle, J & =1, M=+1 \\
\frac{1}{\sqrt{2}}(|\uparrow \downarrow\rangle+|\downarrow \uparrow\rangle), J & =1, M=0 \\
\frac{1}{\sqrt{2}}(|\uparrow \downarrow\rangle-|\downarrow \uparrow\rangle), J & =0, M=0 \\
|\downarrow \downarrow\rangle, J & =1, M=-1 .
\end{aligned}
$$

The ground state has total spin 0 , so the decay from the $J=0$ state (the singlet state) to the ground state is allowed. However, decays from the other three states (the triplet states) to the ground state are forbidden, since they have a total spin of 1. Thus the lifetime of the singlet state is a short 6 ns and the lifetime of the triplet state is a relatively long $1.5 \mu \mathrm{s}$.

The excited electron in the process described by eq. 3.1 has the same spin as before it was excited. However, the excited electron in the process described by eq. 3.2 comes in through recombination, and thus has a spin that is unrelated to that of the ground state electron that it is replacing. So a process that produces a lot of excitons without 


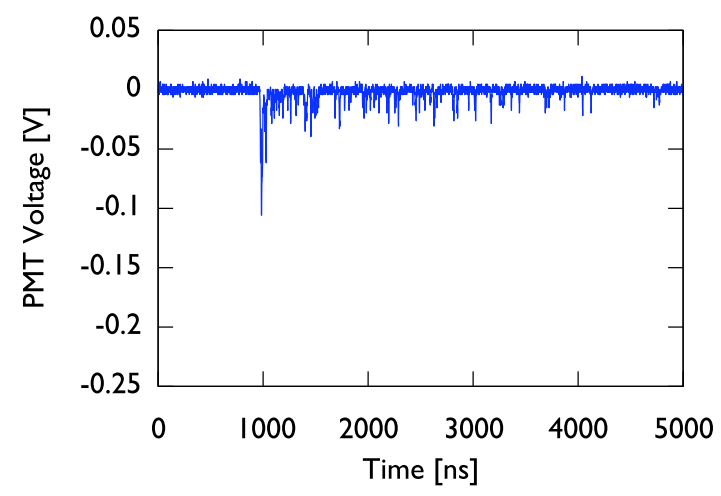

Figure 3.2: A sample waveform for a $\gamma$ absorption in DEAP's liquid argon volume, demonstrating the relatively high population of triplet states at times greater than 1200 ns. Compare with Figure 3.3

an initial ionization of the atom (eq. 3.1) will tend to make a lot of singlets, while a process that produces a lot of ions will tend to make a lot of triplets.

This is important for experiments like DEAP-3600, because interactions that transfer energy primarily to the nucleus (generating "nuclear recoils") tend to produce equal numbers of ions and excitons, while interactions that transfer energy primarily to the electrons (generating "electron recoils") tend to produce a lot more ions than excitons (and therefore more triplets). This gives the experiment the ability to distinguish between an electron recoil and a nuclear recoil. $\beta$ decays and $\gamma$ interactions create electron recoils, while scatters from WIMPs, neutrons, and $\alpha$ particles create nuclear recoils. The fact that the difference in lifetimes for triplet and singlet states is so large makes makes the exclusion of $\beta$ and $\gamma$ events from the WIMP search very straightforward and efficient. Sample plots of light detection as a function of time (often referred to as "waveforms") are shown for a $\gamma$ scatter event (Figure 3.2) and a neutron scatter event(Figure 3.3).

As stated above, the scintillation photons are detected by the 255 LAr PMTs. When a photon hits the PMT's photocathode it liberates an electron through the 


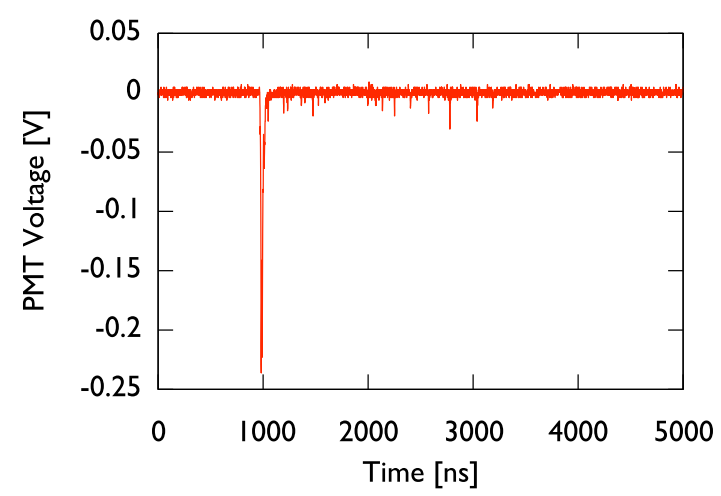

Figure 3.3: A sample waveform for a neutron scatter within DEAP's liquid argon volume, demonstrating the relatively low population of triplet states at times greater than 1200 ns. Compare with Figure 3.2

photo-electric effect. These electrons are known as photo-electrons (PE). Each incident photon can produce up to 1 photo-electron. These photo-electrons trigger an avalanche in the PMT, which creates a voltage spike that is proportional to the number of photo-electrons liberated by the photons. Thus, the size of the combined voltage spike from all PMTs is proportional to the amount of scintillation light in the LAr.

It is fairly straightforward to convert a voltage spike to a total amount of electric charge flowing out of the PMT, provided the behaviour of the electronics is well known. We refer to this as the amount of charge "collected". In order to convert the total charge collected into an approximate number of photo-electrons, we divide the total charge collected by the charge produced by a single photo-electron:

$$
q P E=\sum_{i=0}^{254} \frac{\text { Total }_{i}}{S P E_{i}}
$$

where Total $_{i}$ is the total charge detected by the $\mathrm{i}^{\text {th }} \mathrm{PMT}$ and $S P E_{i}$ is a calibration constant representing the amount of charge produced by a single photo electron in the $\mathrm{i}^{\text {th }}$ PMT. When an event triggers the detector, the PMT signal in the $2.5 \mu$ s before and the $13.5 \mu$ s after the trigger are recorded and saved. 
The SPE constants are calibrated regularly through an optical fibre and LED based light injection system. The ends of the fibres are encased in a small acrylic cylinder, which is attached perpendicularly to the side of the acrylic light guide of one of the LAr-observing PMTs. $435 \mathrm{~nm}$ light is fed into this fibre by an LED, and the intensity of the light is monitored by an avalanche photo-diode. When the LED light enters the light guide, it reflects, and some of it makes its way to the PMT, while approximately $20 \%$ makes its way into the inner detector, where it acts as a diffuse light source for all PMTs within approximately 50 degrees of the light guide. This system was connected to 20 different light guides, spread evenly throughout the liquid argon PMT array. Another 2 connections are present in the neck, and 1 in the water tank for the calibration of the muon veto PMTs.

Before the detector was filled with liquid argon, a "laserball" calibration of all the PMTs was performed [2]. The laserball consisted of an $11 \mathrm{~cm}$ diameter PerFluoroAlkoxy (PFA) flask loaded with $50 \mu \mathrm{m}$ quartz beads suspended in silicon gel. 375, 405, and $445 \mathrm{~nm}$ light was injected into the flask, which diffused the light to make an almost isotropic light source. Measurements showed that the intensity of this source varied no more than $(10 \pm 5) \%$ at all angles.

The values from these calibrations are stored in the DEAP database, along with a large variety of other constants ranging from measurements from slow control systems to input values for afterpulsing models. As a side note, I spent one year as database co-admin, during which time I focused particularly on improving the efficiency and reliability of the database.

The variable that is defined for the purpose of discriminating electron recoils from nuclear recoils is known as "fprompt". fprompt is defined as:

$$
\text { fprompt }=\frac{\int_{-28 n s}^{60 n s} P E(t) d t}{\int_{-28 n s}^{10 \mu s} P E(t) d t}
$$




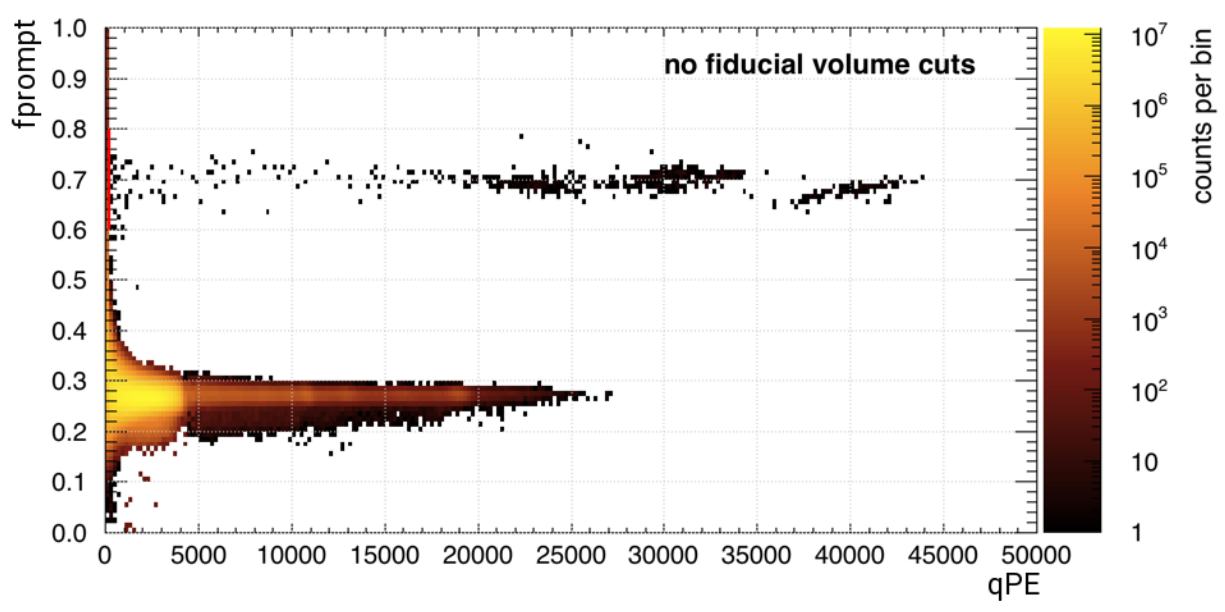

Figure 3.4: The distribution of LAr scintillation events in DEAP-3600. The electron recoil band can clearly be seen with an fprompt value between 0.2 and 0.3 . The nuclear recoil band lies between 0.6 and 0.8 fprompt.

where $P E(t)=\sum_{i=0}^{254} Q_{i}(t) / S P E_{i}$. By this definition, nuclear recoil events with large populations of short-lived singlets will have a high fprompt, while electron recoil events with large populations of longer-lived triplets will have a lower fprompt. An example plot showing the distribution of events in fprompt and qPE space is shown in Figure 3.4. The nuclear recoil band is at fprompt $\sim 0.7$ and the electron recoil band is at fprompt $\sim 0.3$. Differentiating between electron and nuclear recoils in this way is referred to as pulse-shape discrimination (PSD).

A summary of where different classes of events appear in the (fprompt, qPE) plane is given in Table 3.1 .

\subsection{PMT and Digitizer Saturation}

The PMTs are read out by a data acquisition system (DAQ) that reads out the PMT signal, processes it, converts the analog voltage signals from the PMTs to a digital signal that can be stored on a hard drive, and finally saves it to disk. The DAQ is illustrated in Figure 3.5. The V1720 and V1740 digitizer modules are responsible for 


\begin{tabular}{c|c|c}
\hline Event Type & fprompt & qPE \\
\hline WIMPs & high & low \\
LAr $\alpha$ Decays & high & high \\
Surface $\alpha$ Decays & high & low - high \\
Neutrons & high & low - medium \\
$\beta$ Decays & low & low - high \\
Gammas & low & low - high \\
\hline
\end{tabular}

Table 3.1: Qualitative summary of the regions in the (fprompt, qPE) plane where various classes of events would appear. Here low and high fprompt are defined as 0.2-0.3 and 0.6-0.8 respectively. Low, medium, and high $\mathrm{qPE}$ are defined as 100-500, 500-6000, and 6000-70000 respectively.

converting the analog signal to a digital one. The V1720s have a $250 \mathrm{MS} / \mathrm{s}$ sampling rate, and the V1740s have a $62.5 \mathrm{MS} / \mathrm{s}$ sampling rate. The V1720s are used for the primary dark matter search. The V1740s deal with large PMT pulses that would saturate the V1720s. The pulses are therefore attenuated by a factor of 10 and broadened before being sent to the V1740s.

Unfortunately, the trigger settings for the V1740s was corrupted by a software glitch during the first year of data taking. This meant that only the V1720 data was fit for use. The saturation of the V1720s did not affect the WIMP search directly, since the PMT pulses in the WIMP ROI are not large enough to saturate the V1720 digitizers. However, the full energy $\alpha$ decays in the LAr produce signal that are two orders of magnitude larger than the signals in the WIMP ROI. In addition to digitizer saturation, PMTs exhibit non-linear behaviour when high numbers of PE are produced.

When a digitizer saturates, the signal simply gets cut off, or "clipped", as shown in Figure 3.6. When a PMT receives high quantities of light, the ratio of the magnitude of the PMT voltage spike and the amount of light incident on the PMT photocathode is reduced, thus making the signal from an interaction such as an $\alpha$ decay look less 


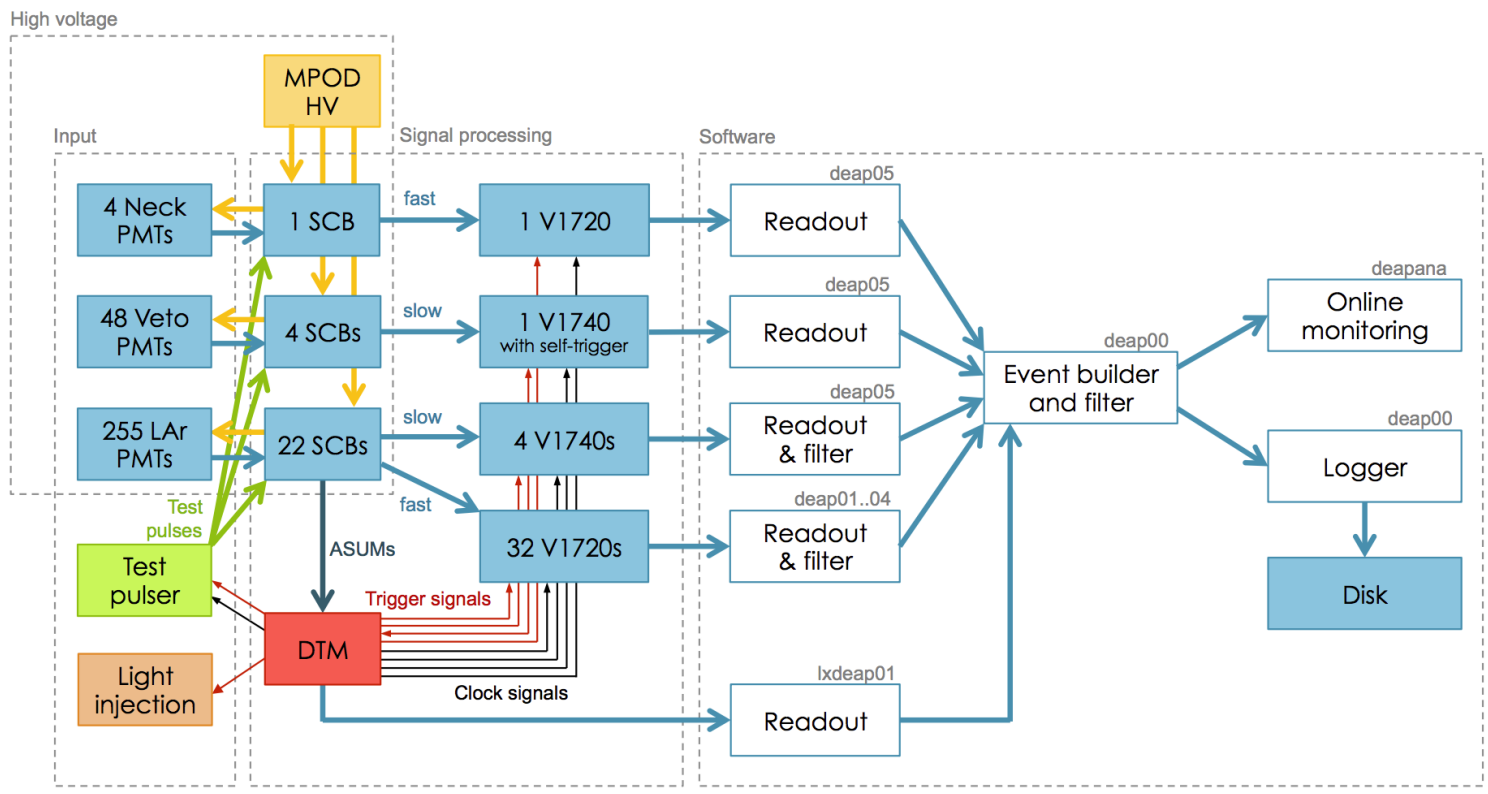

Figure 3.5: Illustration of DEAP DAQ system. Hardware is shaded and software is white. [2]

energetic than it otherwise would.

Events that occur near the LAr - acrylic vessel (AV) interface will concentrate most of their light in a few PMTs, while light from events that occur near the centre of the LAr volume will be fairly evenly distributed around the detector. This is illustrated in Figure 3.7. As can clearly be seen in this figure, the PMTs with the most charge have several orders of magnitude more charge in the case where the event is situated close to the edge of the LAr volume. Events close to the edge will therefore be more strongly affected by digitizer clipping and PMT non-linearity than events that occur far from the edge. This broadens the $\alpha$ decay peaks in the energy spectrum, and causes them to overlap.

In addition to the effect on the reconstructed energy of high energy events, there is also a significant effect on the fraction of total light in the prompt time window (fprompt). Since the singlet lifetime is so short (6 ns), a large fraction of the total light from an event is produced very quickly. PMT non-linearity and digitizer clipping 

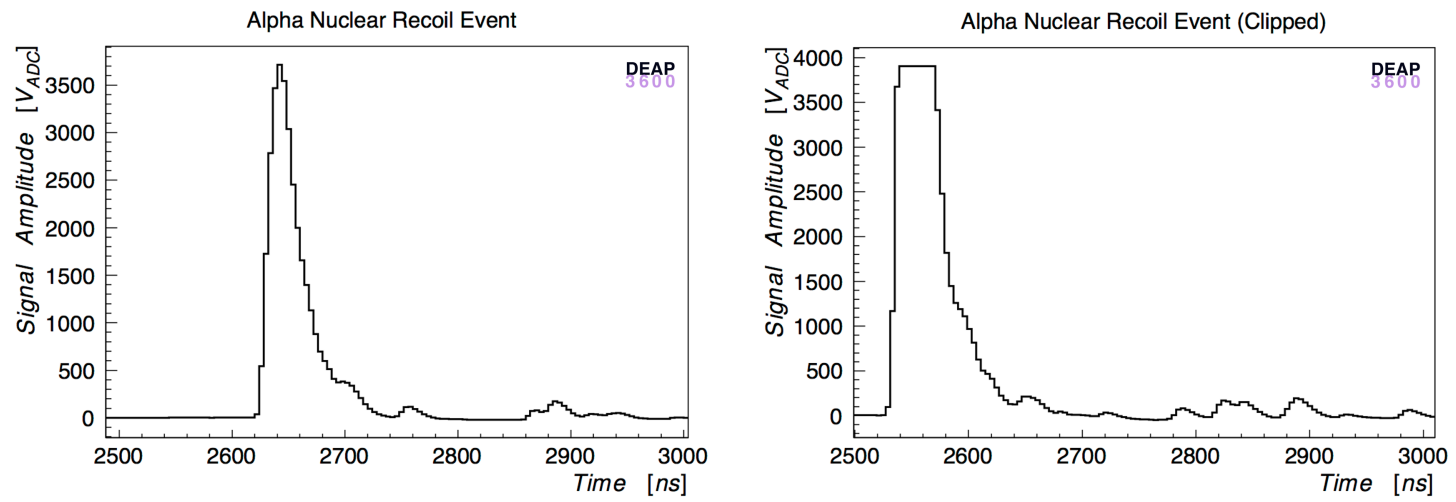

Figure 3.6: Sample waveforms for $\alpha$ particle scatters in liquid argon. On the left is a normal waveform, and on the right is a waveform exhibiting digitizer clipping from a higher energy event.
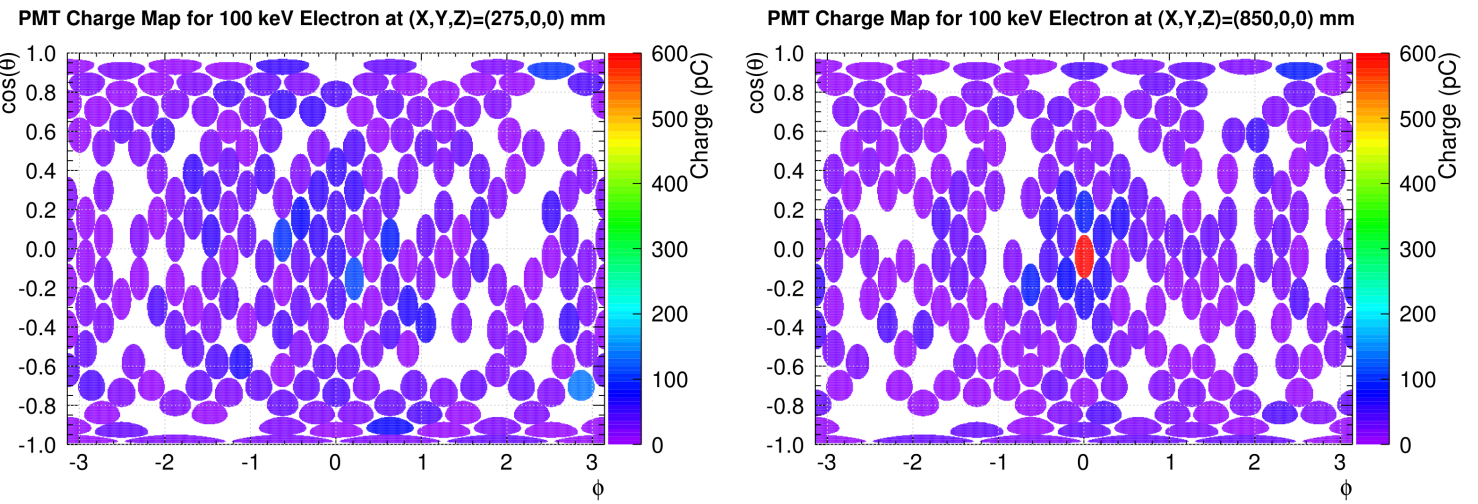

Figure 3.7: Monte Carlo generated PMT hit patterns for a $100 \mathrm{keV}$ electron at $275 \mathrm{~mm}$ from the centre of the LAr volume (left) and $1 \mathrm{~mm}$ from the LAr-AV interface (right). 


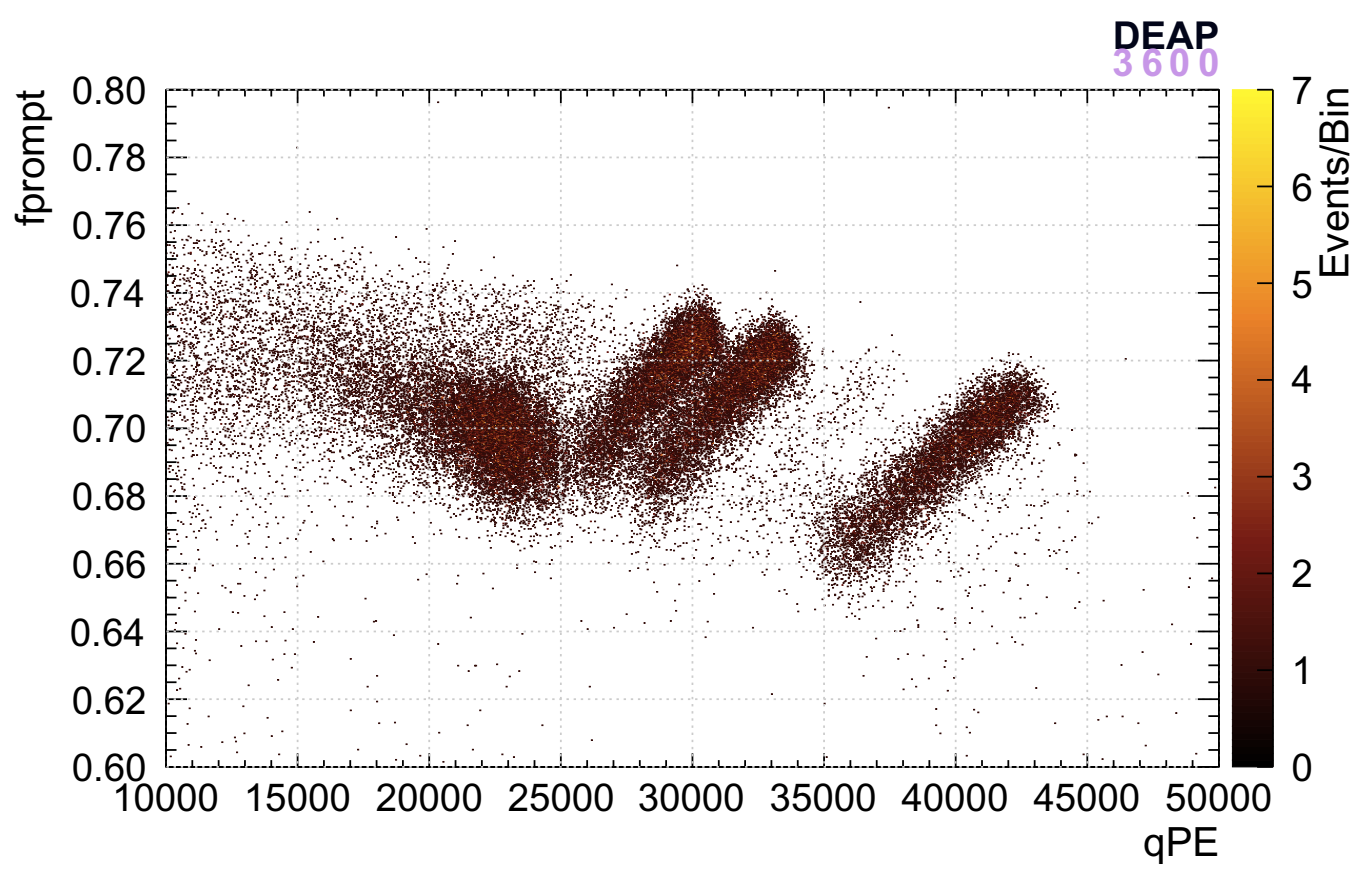

Figure 3.8: $\alpha$ decay peaks in fprompt vs qPE. The peaks are slanted due to digitizer clipping and PMT non-linearity.

will therefore have a much stronger effect on PMT pulses in the prompt time window. This reduces the fprompt for high energy events that occur close to the edge of the detector. An example of the effects on both fprompt and $\mathrm{qPE}$ is shown for $\alpha$ decays in Figure 3.8 .

\subsection{Datasets}

Several configurations exist for the recording of data in DEAP-3600. However, only AmBe calibration data and "physics" data will be relevant for this report.

\subsubsection{AmBe Calibration Data}

AmBe calibration data is taken when an AmBe source is deployed in one of the calibration tubes visible in Figure 3.9 . 


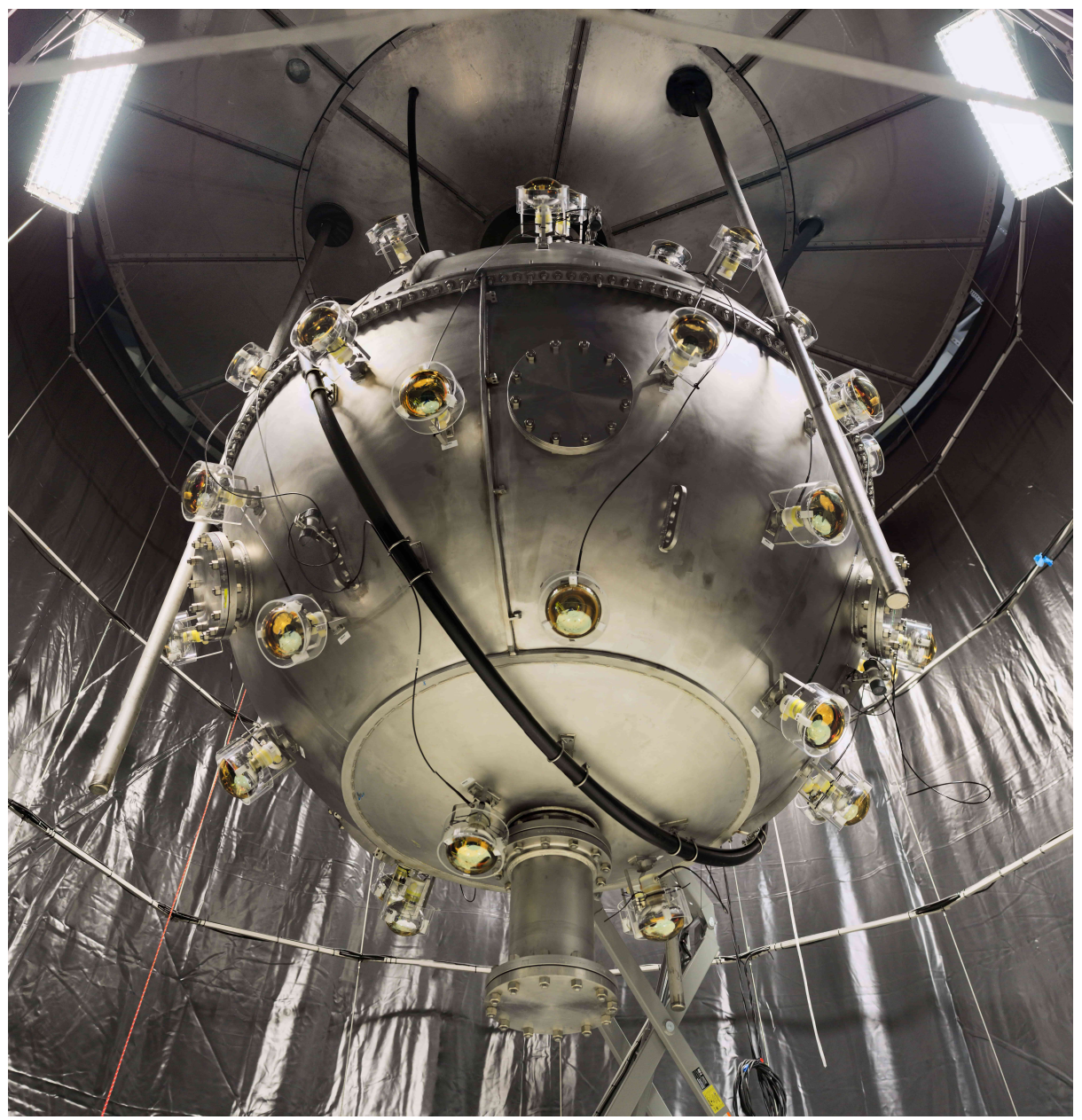

Figure 3.9: Image of the DEAP-3600 detector after being encased in the steel shell. The AmBe source was placed within the straight steel tubes on either side of the detector, which are known as "Cal" tubes. 
The AmBe calibration source is composed of a mixture of ${ }^{241} \mathrm{Am}$ and ${ }^{9} \mathrm{Be}$. The ${ }^{241} \mathrm{Am}$ atoms produce $\alpha$ particles when they decay, which can then be absorbed by the ${ }^{9} \mathrm{Be}$ to produce ${ }^{13} \mathrm{C}$ in an excited nuclear state. ${ }^{13} \mathrm{C}$ then quickly decays to ${ }^{12} \mathrm{C}$ by emitting a neutron. This process is summarized in eq. 3.6 .

$$
\begin{aligned}
{ }^{241} \mathrm{Am} & \rightarrow{ }^{237} \mathrm{~Np}+\alpha(5.4-5.5 \mathrm{MeV})+\gamma(59 \mathrm{keV}) \\
\alpha+{ }^{9} \mathrm{Be} & \rightarrow{ }^{13} C^{*} \\
{ }^{13} C^{*} & \rightarrow{ }^{12} C^{*}+\mathrm{n} \\
{ }^{12} C^{*} & \rightarrow{ }^{12} \mathrm{C}+\gamma
\end{aligned}
$$

This neutron can then propagate through the detector, where it will likely scatter and lose energy. As it loses energy, the neutron capture cross section becomes significant, and the neutron will eventually be captured by an atom. The neutron's binding energy is then released in the form of one or more $\gamma$ particles. In the case of the release of several $\gamma$ particles, the energies of the $\gamma$ s follow a spectrum determined by the nuclear energy levels of the newly created isotope. The neutron capture $\gamma$ spectrum has been measured for several isotopes, and is available in online libraries, such as [53|. These known spectra are included in GEANT4, which models the neutron capture process in its simulations. In addition to the indirect creation of neutron capture $\gamma \mathrm{s}$, the AmBe source provides an additional two $\gamma$ lines from the de-excitation of the final state nucleus of the ${ }^{12} \mathrm{C}$ isotope produced within the AmBe source.

The final line in eq. 3.6 can take on one of three forms based on the internal energy levels of the ${ }^{12} \mathrm{C}$ nucleus (see Figure 3.10. The most common is the emission of a single $4.439 \mathrm{MeV} \gamma$, with a branching ratio of approximately $61 \%$. The second most common case is where no $\gamma$ is released at all, which occurs with a $36 \%$ branching ratio. The least common case is the emission of both a $4.439 \mathrm{MeV}$ and a $3.215 \mathrm{MeV}$ 


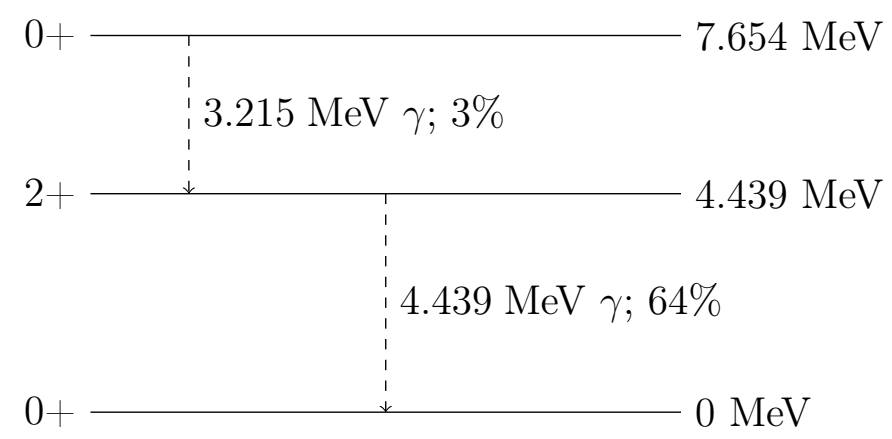

Figure 3.10: Energy level diagram of the ${ }^{12} \mathrm{C}$ nucleus, showing only the first two excited states, which are responsible for the $\gamma$ rays produced by the AmBe neutron source. The spins are shown on the left, and the level energies are on the right. The fraction of the time a $\gamma$ is produced is expressed as a percent.

$\gamma$. The $4.439 \mathrm{MeV} \gamma$ is important for calibration, as it produces a high-intensity mono-energetic peak in the energy spectrum.

\subsubsection{Low Background ("Physics") Data}

Low background (or "physics") data is taken with no sources near the detector and is where rare interactions (such as from WIMPs or axions) could be detected. In this report, "physics data" refers to this standard operating mode of the detector. The DEAP-3600 detector has been filled twice. The first fill short-lived, as the detector developed a leak when a seal failed. The leak resulted in radio-pure nitrogen entering the main target volume. The detector was emptied and refilled, but only up to a level of $551 \mathrm{~mm}$ above the equator. This analysis exclusively uses second fill data, which was taken between November 2016 and March 2020. The $\alpha$ studies described in Chapter 5 were performed on the 247.17 live days of data taken from November 2016 to October 2017, inclusive. The radiogenic neutron study (Chapter 6) and axion search (Chapter 7) were performed on the full 802 live day dataset, taken between November 2016 and March 2020. 


\subsection{Software}

DEAP-3600 uses ROOT [54], RAT [55], and GEANT4 [56]. ROOT is an extensive analysis toolkit written specifically for physicists in $\mathrm{C}++$ and python. This analysis used ROOT version 5.34.36. GEANT4 is a particle simulation toolkit that models the paths of particles through matter. GEANT4 version 9.6.p02 was used for this analysis. RAT is a wrapper that sets up the GEANT4 simulation based on the values set in the RAT database (DB), runs and processes the simulation based on the configuration specified in a user-provided macro, and stores the data in the ROOT file format for easy downstream analysis. RAT is currently not compatible with newer versions of GEANT4, but extensive work is underway to change this.

RAT is able to simulate the detector electronics in detail. GEANT4 simulates the physics of particle propagation within the detector, and records the number of photons that are absorbed by the PMTs. RAT then takes this number and uses measured efficiency values to determine how many photo-electrons (PEs) are produced. This produces a simulated voltage spike, with the size of the spike determined by measured single photo-electron (SPE) values for the run being simulated. The electronic signal processing is also simulated, along with effects such as dark noise and afterpulsing. The production of photons when energy is deposited in the LAr is modelled by a class (GLG4) built into RAT. This class models the triplet and singlet production based on the ID of the particle that deposited energy in the LAr, allowing for a good model of the fprompt variable. 


\section{Chapter 4}

\section{Radioactivity in DEAP-3600}

\section{1 $\alpha$ Decay Backgrounds}

In order to perform a low background WIMP search, it is essential that the radioactive backgrounds in DEAP-3600 be understood as well as possible. One such background is $\alpha$ decay.

The dominant $\alpha$ decay chains in DEAP-3600 are the ${ }^{238} \mathrm{U}$ and ${ }^{232}$ Th series. These are shown in Figures 4.1 and 4.2 respectively. $\alpha$ decaying isotopes from these chains are present in practically all detector components. These decays generally manifest in the data as Cerenkov events, $(\alpha, n)$ events, neck events, surface events, or bulk LAr events. The characteristics of these events are summarized in Table 4.1.

\begin{tabular}{c|c|c}
\hline Event & Source & Description \\
\hline Cerenkov & $\gamma$ produced as result of $\alpha$ decay & Very low energy \\
$\alpha, \mathrm{n}$ & $(\alpha, \mathrm{n})$ on component & Nuclear recoil and/or capture $\gamma$ \\
Neck & $\alpha$ decay in neck & Low energy with shadowed hit pattern \\
Surface & $\alpha$ decay on LAr-acrylic interface & Degraded energy spectrum \\
Bulk & $\alpha$ decay in LAr volume & High energy peak \\
\hline
\end{tabular}

Table 4.1: Qualitative overview of selected data event types that could be caused by $\alpha$ decay.

Cerenkov light can be produced when a high energy charged particle travels faster 


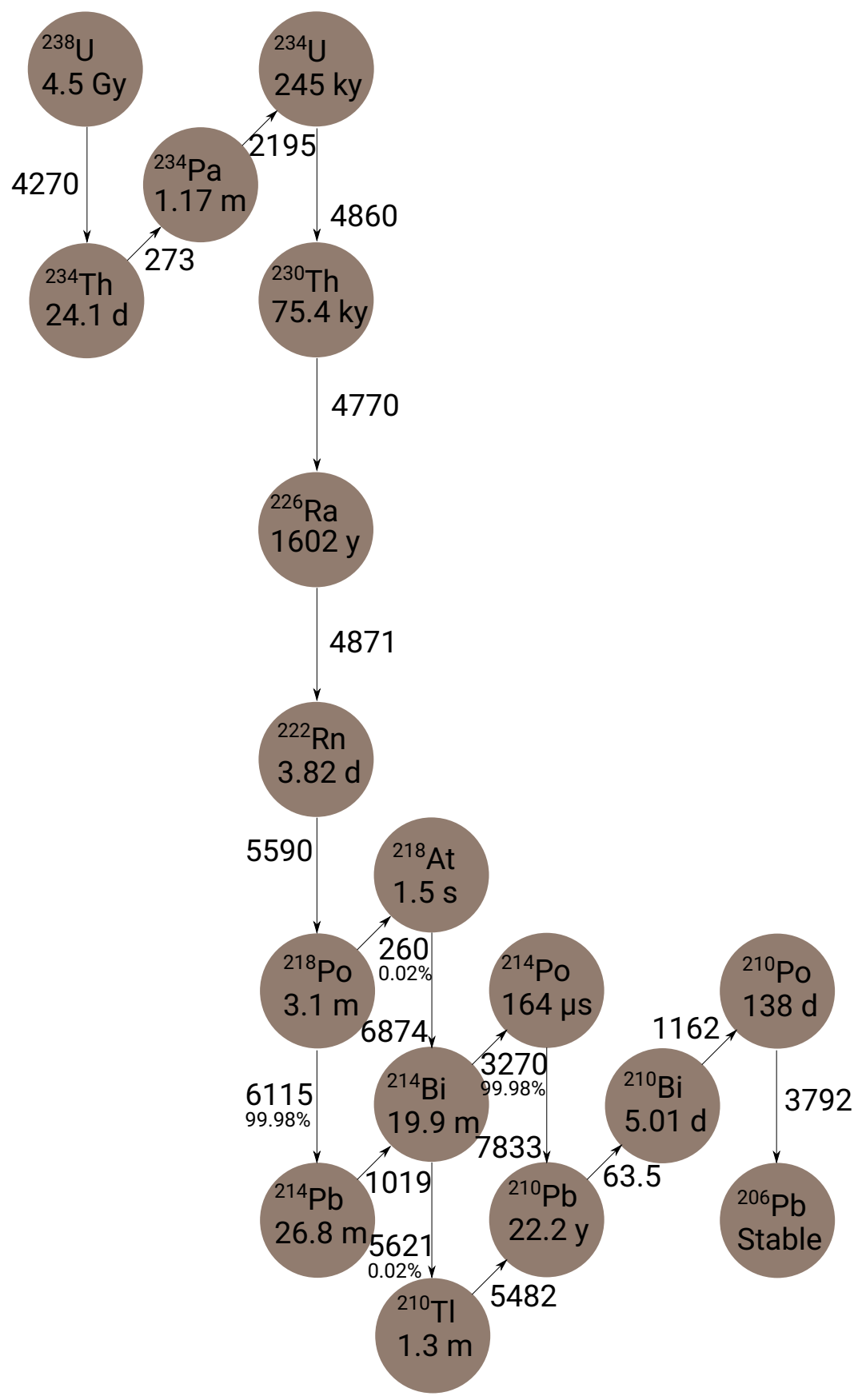

Figure 4.1: ${ }^{238} \mathrm{U}$ decay chain. Downward pointing arrows represent $\alpha$ decays and upper-right pointing arrows represent $\beta$ decays. The numbers closest to the arrowheads represent the Q-value in $\mathrm{eV}$. Only decays with a branching ratio of at least $1 \%$ are shown. The half-life of each isotope is listed under its name. 


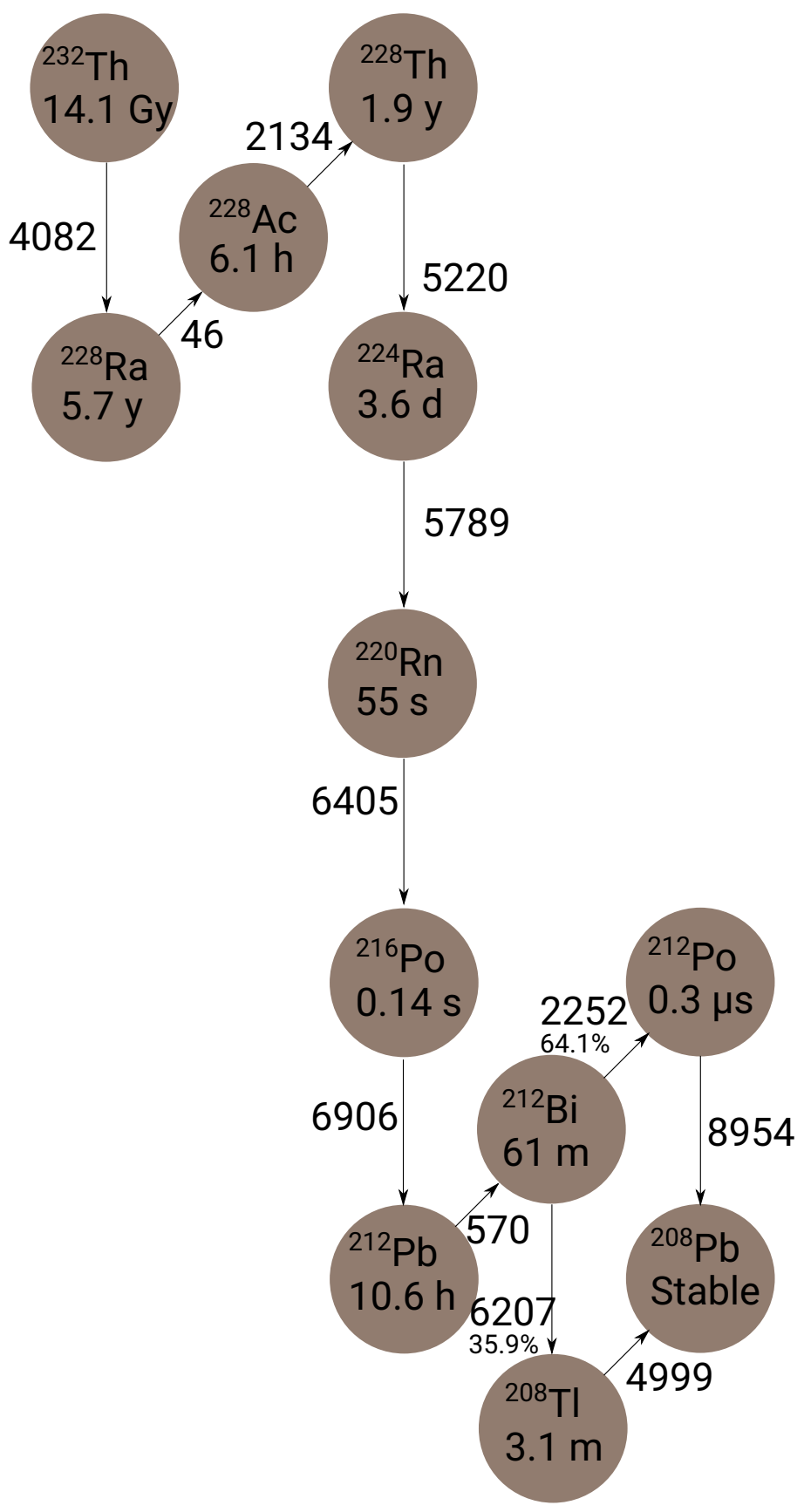

Figure 4.2: ${ }^{232} \mathrm{Th}$ decay chain. Downward pointing arrows represent $\alpha$ decays and upper-right pointing arrows represent $\beta$ decays. The numbers closest to the arrowheads represent the Q-value in $\mathrm{eV}$. Only decays with a branching ratio of at least $1 \%$ are shown. The half-life of each isotope is listed under its name. 
than the phase velocity of light in a medium (given by $c / n$ where $c$ is the speed of light in a vacuum, and $n$ is the index of refraction of the medium). This can happen in DEAP-3600 if an electron from a high energy $\beta$ decay passes through the acrylic, for example. Cerenkov can also be induced when neutral particles, such as $\gamma$ rays, pass through a material and ionize high energy electrons which then propagate through the medium faster than the phase velocity of light in that medium. These events often have very high fprompt values, and deposit rather low amounts of energy in the detector, but occasionally they can have energy and fprompt values that are within the WIMP region of interest (WIMP ROI), making these an important background to understand.

It is rare, though possible, for $\alpha$ decays to simultaneously produce a $\gamma$ ray. However, $\gamma$ rays are common products of $\beta$ decays, and it is very common for a $\beta$ decay to occur between $\alpha$ decays within the same decay chain. So measuring the activity of $\alpha$ decaying isotopes can also inform estimates of the frequency of Cerenkov backgrounds due to $\beta$ decays.

$\alpha$ particles can occasionally be absorbed by various nuclei within detector components. This will sometimes result in an unstable nuclear state, which then quickly emits a neutron. These neutrons can provide a dangerous background, since there can sometimes be very little intermediate material shielding the inner detector volume. These neutrons have high fprompt, and qPE values ranging from 100 to 5000, which overlaps with the WIMP ROI at 100 to 240 qPE. A proper understanding of the rate of these neutrons is essential to the overall background model.

Another very dangerous background for DEAP-3600 is $\alpha$ particles produced in the neck. Possibly due to the (unconfirmed) presence of a layer of liquid argon on the surface of the neck, $\alpha$ particles produced by decays on the surface can undergo partial scintillation, which, together with shielding by components in the neck, can bring the energy down to the level of the WIMP ROI. The shadowing provides an 
asymmetric pattern in the PMT hit map, however, and cuts have been developed to exclude the vast majority of these events from the dataset. Extensive work has been done within the collaboration to properly model this background.

$\alpha$ particles produced by decays just under the TPB or acrylic surface of the inner detector volume can be another source of low energy, high fprompt backgrounds. An $\alpha$ particle will typically lose a significant amount of energy as it passes through a portion of the surface before entering the liquid argon (see illustration in Figure 4.3). While most of the events will still have energies above the WIMP ROI, it is still possible for a very small fraction of them to leak into the ROI and mimic a dark matter signal.

$\alpha$ decays within the bulk of the liquid argon are not directly important for the WIMP background model, but a knowledge of the overall rate is still a very important constraint on the overall $\alpha$ background expectation in other regions of the detector, especially since these isotopes could also plate out on the surface of the inner detector. They also provide an important constraint on the overall activity of the decay chains to which they belong, constraining the $\beta$ decay expectation, and the backgrounds they produce. They have also been used as an in-situ high energy calibration source.

In this work, the rates of ${ }^{222} \mathrm{Rn},{ }^{218} \mathrm{Po},{ }^{214} \mathrm{Po},{ }^{220} \mathrm{Rn},{ }^{216} \mathrm{Po},{ }^{212} \mathrm{Bi}$, and ${ }^{212} \mathrm{Po}$ decay backgrounds will be determined.

\subsection{Radiogenic Neutrons}

As described previously, $(\alpha, n)$ neutrons are potentially a very dangerous background in a WIMP search. Getting an accurate estimate on their rate is thus very important.

Fortunately, neutron scatters are usually followed shortly after by a neutron capture interaction, in which the neutron is absorbed by a nucleus, and the remaining energy is emitted in the form of a $\gamma$ ray. This $\gamma$ ray will deposit energy in the liquid 


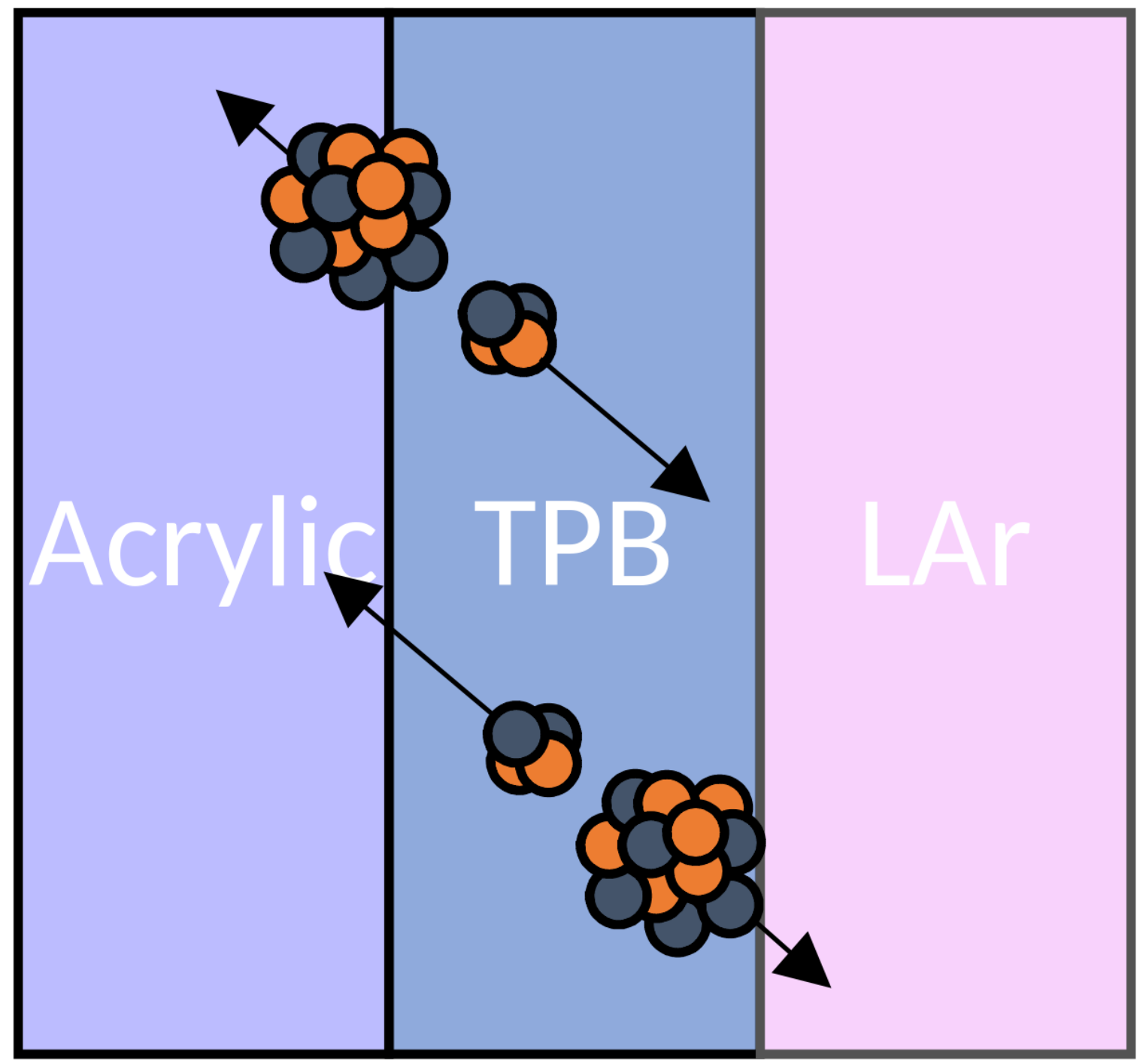

Figure 4.3: Illustration demonstrating the two types of surface $\alpha$ decays that can result in events with degraded reconstructed energy. If an $\alpha$ particle loses enough energy while passing through enough TPB, the energy deposited in the LAr could be low enough for the event to mimic a dark matter particle. Similarly if the nucleus recoils into the LAr from the TPB, the event could mimic a dark matter signal. 
argon some fraction of the time, producing a high energy, low fprompt signal. These correlated low fprompt events can be used as a tag for neutron scatters, thus giving us a way to estimate the radiogenic neutron scatter rate in the liquid argon.

\subsection{Argon Decay}

The liquid argon target is itself radioactive. In fact, the ${ }^{39} \mathrm{Ar} \beta$-decay event count dominates the detector datasets with a rate of $3120 \pm 190 \mathrm{~Hz}$ [1]. These electron-recoil events are excluded from the WIMP search using the fprompt variable described in the previous chapter. 


\section{Chapter 5}

\section{Characterization of $\alpha$ Decays}

As stated in the introduction, the uranium and thorium decay chains are the dominant sources of radioactive backgrounds in DEAP-3600 and it is essential that they be understood for the WIMP dark matter search. Radioactive isotopes can enter the detector during filling, or be present within the detector components themselves. After the detector was isolated from the external environment, short lived isotopes quickly decayed away. The only source of these isotopes would then be the radioactive decays of other isotopes higher in the decay chain. The rate of decay of these short lived isotopes would therefore be equal to the rate of decay of their parents, multiplied by any relevant branching ratios. This state is known as "secular equilibrium". Since DEAP-3600 has been operating for a few years, secular equilibrium applies here for decays below radium in the thorium chain, and between radium and ${ }^{210} \mathrm{Po}$ in the uranium chain.

This chapter will summarize my efforts to measure the activities of ${ }^{222} \mathrm{Rn},{ }^{218} \mathrm{Po}$, ${ }^{214} \mathrm{Po},{ }^{220} \mathrm{Rn},{ }^{216} \mathrm{Po},{ }^{212} \mathrm{Bi}$, and ${ }^{212} \mathrm{Po}$ within the liquid argon bulk and the rate of ${ }^{214} \mathrm{Po}$ on the inner surface of the detector. I will also constrain the amount of ${ }^{214} \mathrm{Po}$ in the neck and on dust-like events. It is important to understand all sources of $\alpha$ backgrounds in DEAP-3600, as these nuclear recoil events could mimic a WIMP signal if the amount of light produced is sufficiently low. 
Note that all of the analysis in this chapter was performed on DEAP-3600's 247.17day dataset, and the results of this analysis were published in [1]. All uncertainties quoted are statistical only, unless otherwise noted.

\subsection{Cut Variables}

Cuts are applied to the data prior to running the analysis code. These cuts remove unwanted events from the dataset while keeping as many events of interest as possible. This section will present the meanings of the cuts that are used in this thesis.

- fmaxpe: The fraction of total photo-electrons in an event that are collected by the PMT with the highest amount of collected charge. A high fmaxpe value indicates that most of the light was deposited in a single PMT, while a low fmaxpe value indicates that the light was deposited relatively evenly throughout the detector. Values can range between 1/255 and 1.

- Standard low level cuts: Removes events that exist due to the periodic $40 \mathrm{~Hz}$ trigger or some other calibration trigger, contain electronic noise in an unplugged channel, or have a readout error flag set. See Appendix A for more details.

- Standard pileup cuts: Removes events that are likely to be pileup according to various algorithms. See Appendix A for more details.

- mblikelihoodX, mblikelohoodY, mblikelihoodZ: Reconstructed X, Y, and $\mathrm{Z}$ coordinates of the location of an event in the LAr volume. The algorithm that produces this number is known as "mblikelihood".

- MBR or mblikelihoodR:

$\sqrt{\text { mblikelihoodX }^{2}+\operatorname{mblikelihood}^{2}+\operatorname{mblikelihoodZ}}$ 
- nSC: A qPE-like variable that attempts to remove PMT afterpulsing through the use of a statistical model.

- rprompt: An fprompt-like variable. rprompt attempts to remove PMT afterpulsing in the same way as nSC. Afterpulsing is caused by the ionization of a residual gas atom within the PMT during the charge avalanche process that produces a voltage spike during normal operation. This ionized atom eventually triggers a second avalanche after travelling to the photocathode or first dynode, resulting in the presense of additional pulses after an initial photon-induced pulse.

- fmaxpeprompt: Fraction of photo-electrons in the prompt window in an event that are collected by the PMT with the most prompt charge.

- qPE_60: Amount of qPE in the first 60 ns of an event's waveform.

Four "box cuts" were defined (see Section A to select $\alpha$ events in the LAr bulk. These will often be referred to as the "full energy" events.

\subsection{Determining the Activities of ${ }^{222} \mathrm{Rn},{ }^{218} \mathrm{Po},{ }^{214} \mathrm{Po}$, ${ }^{220} \mathrm{Rn},{ }^{216} \mathrm{Po}, \&{ }^{212} \mathrm{Bi}$}

\subsubsection{Introduction}

DEAP was optimized to look for low energy WIMP signals, so the high energies of the $\alpha$ decay events within the detector are smeared due to clipping of the PMT signal by the digitizers. This smearing, combined with the proximity of the energies of some of the $\alpha$-decaying radioisotopes to one another, results in a large amount of overlap between the populations (Figure 5.1). And since this smearing is non-Gaussian, it 
is very difficult to estimate the uncertainty on the rates of individual $\alpha$ populations using the reconstructed energy alone and to know the amount of leakage across cut boundaries. There is also significant pileup of $\alpha$ decays with ${ }^{39} \mathrm{Ar}$ events. About $4 \%$ of $\alpha$ decays will be followed by an ${ }^{39} \mathrm{Ar}$ decay within the $\alpha$ decay's $13.5 \mu$ s window, and an additional $4 \%$ of $\alpha$ decays will occur within an ${ }^{39} \mathrm{Ar}$ event's $13.5 \mu$ s window. This results in an additional degree of cross-contamination of $\alpha$ populations that neighbour each other in energy. Due to the nature of the pileup, it cannot be modelled well using an analytic function.

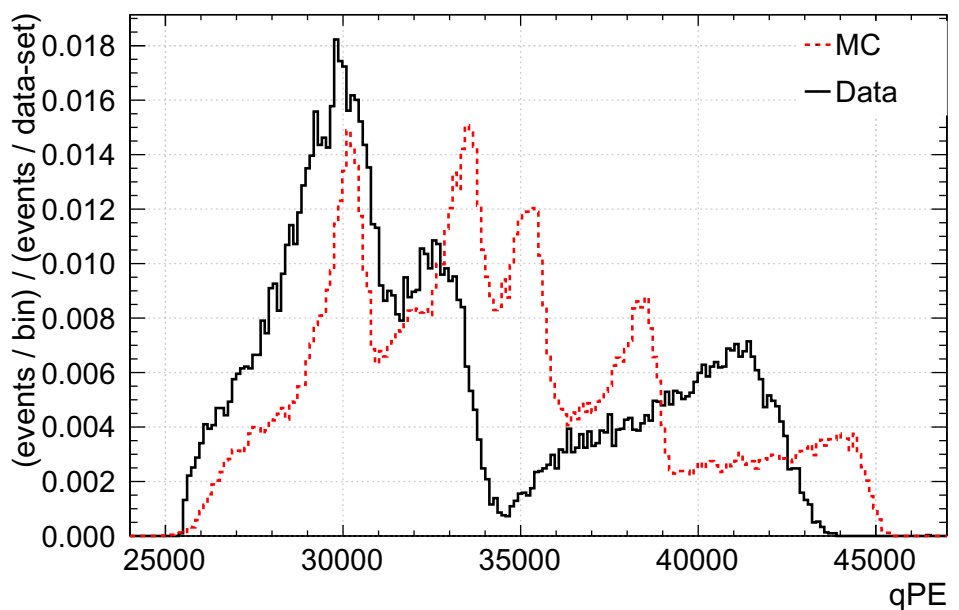

Figure 5.1: Monte Carlo of $\alpha$ decays in the LAr bulk (red) and data (black) qPE distributions before correcting the energy or fitting the normalizations. The Monte Carlo distribution has a higher amplitude due to more statistics than the data sample. The peaks are overlapping due to digitizer clipping (which is modeled in the $\mathrm{MC}$ ).

As discussed in Section 3.3 digitizer clipping and PMT non-linearity have a significant effect at $\alpha$ decay energies. The amount of clipping and non-linear effects is dependant on the amount of light in the individual PMTs rather than the overall amount of light in the detector. Events close to the surface of the detector deposit a large fraction of the light in a few PMTs, and will thus be more strongly affected by 
digitizer clipping and PMT non-linearity than events close to the centre of the detector. This results in a lower reconstructed event energy ( $\mathrm{qPE}$ ) for events close to the surface compared to events close to the centre of the detector. Since digitizer clipping and PMT non-linearity tend to occur exclusively in the prompt window, full energy $\alpha$ decays near the surface will have a lower fprompt than full energy decays near the centre. Combined, these two effects cause the full energy $\alpha$ peaks to be spread out in fprompt vs qPE space (see Figure 5.3). This spread approximately follows a positive linear slope, with the position of individual events in these distributions determined by their proximity to the detector surface.

One way to try to estimate the activity of each population is to draw lines around each population in fprompt vs qPE space and count the number of events in each box (shown in Figure 5.3). However, this approach has some drawbacks including crosscontamination due to pileup with ${ }^{39} \mathrm{Ar}$, and cross-contamination and leakage out of the boxes due to the proximity of the peaks in fprompt vs qPE space. Also note that the ${ }^{218} \mathrm{Po}(6.115 \mathrm{MeV}),{ }^{220} \mathrm{Rn}(6.405 \mathrm{MeV})$ and ${ }^{212} \mathrm{Bi}(6.207 \mathrm{MeV})$ are all overlapping the same "box", with ${ }^{218}$ Po dominating by 2 orders of magnitude. Quantifying the amount of overlap between regions was one of the primary motivations for performing a fit.

The Monte Carlo can demonstrate the impacts of these effects to a point, but differences between the Monte Carlo model and the detector prevent a direct comparison between the data and Monte Carlo by affecting the widths of the distributions, their fprompt, and their energies. Since starting work on this fit, the Monte Carlo has been tuned to match the data in this region (for the ${ }^{222} \mathrm{Rn}$ and ${ }^{218} \mathrm{Po}$ peaks), but PMT non-linearity effects are still not modeled, resulting in a mismatch between data and Monte Carlo above ${ }^{218}$ Po energies (Figure 5.2). A correction has been developed to allow the Monte Carlo to be used in performing a fit to the data, as will be discussed in the following sections. The purpose of the fit is to extract the activity of $\alpha$ events 


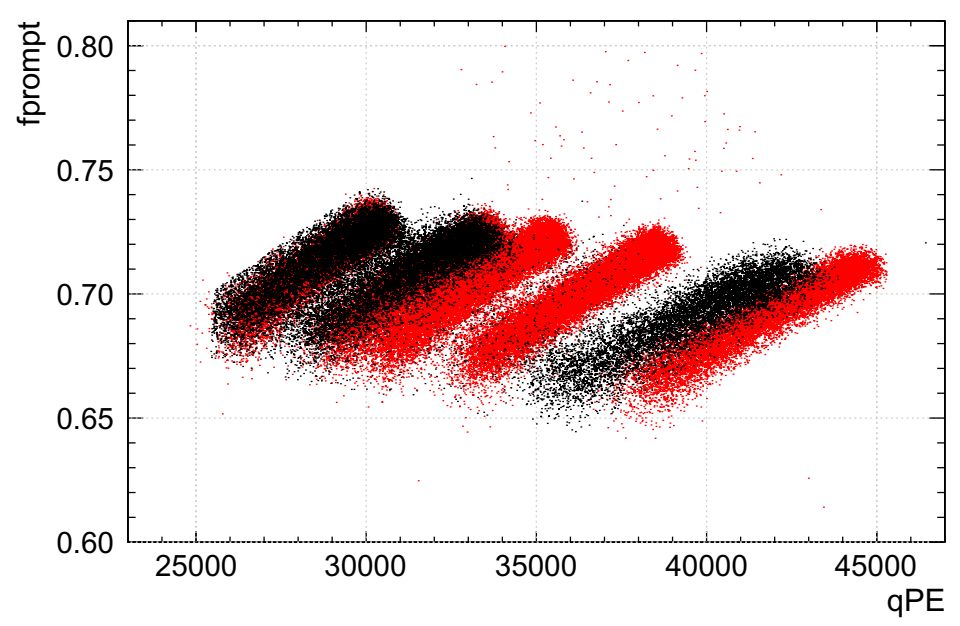

Figure 5.2: The Monte Carlo (red) and data (black) fprompt vs qPE distributions before fitting the normalizations. The populations are (from left to right) ${ }^{222} \mathrm{Rn}$, ${ }^{218} \mathrm{Po},{ }^{220} \mathrm{Rn},{ }^{216} \mathrm{Po}$, and ${ }^{214} \mathrm{Po}$. The ${ }^{222} \mathrm{Rn},{ }^{218} \mathrm{Po}$, and ${ }^{214} \mathrm{Po}$ peaks dominate in the data.

in the $\mathrm{U}$ and $\mathrm{Th}$ chains, specifically ${ }^{222} \mathrm{Rn},{ }^{218} \mathrm{Po},{ }^{220} \mathrm{Rn},{ }^{216} \mathrm{Po}$, full energy ${ }^{214} \mathrm{Po}$, surface ${ }^{214} \mathrm{Po}$, and ${ }^{212} \mathrm{Bi}$.

\subsubsection{Overview of approach}

\section{Preparing Monte Carlo Ntuples for Fit}

The Monte Carlo that was used in this study is described in table 5.1 .

Prior to running the fitter, the first step is to add branches to the Monte Carlo ntuples with some truth information. Each branch in an ntuple stores the value of one variable for each entry (i.e., MC event). These branches are currently labelled as:

- Alpha - One of 222, 220, 218, 216, 214, or 212. Identifies the event as being produced by a decay of one of ${ }^{222} \mathrm{Rn},{ }^{218} \mathrm{Po},{ }^{220} \mathrm{Rn},{ }^{216} \mathrm{Po},{ }^{214} \mathrm{Po}$, or ${ }^{212} \mathrm{Bi}$.

- Count - Which decay in the Monte Carlo this corresponds to. This is needed because it is possible for a decay to result in more or less than one ntuple entry. 


\begin{tabular}{|c|c|}
\hline Description & Number of Primary Monte Carlo Events Generated \\
\hline${ }^{222} \mathrm{Rn}$ & 20000 \\
\hline${ }^{220} \mathrm{Rn}$ & 20000 \\
\hline${ }^{218} \mathrm{Po}$ & 20000 \\
\hline${ }^{216} \mathrm{Po}$ & 20000 \\
\hline${ }^{214} \mathrm{Bi}$ and ${ }^{214} \mathrm{Po}$ & 31496 \\
\hline${ }^{212} \mathrm{Bi}$ & 20000 \\
\hline${ }^{222} \mathrm{Rn}$ and ${ }^{39} \mathrm{Ar}$ & 2500 \\
\hline${ }^{220} \mathrm{Rn}$ and ${ }^{39} \mathrm{Ar}$ & 2500 \\
\hline${ }^{218} \mathrm{Po}$ and ${ }^{39} \mathrm{Ar}$ & 2500 \\
\hline${ }^{216} \mathrm{Po}$ and ${ }^{39} \mathrm{Ar}$ & 2500 \\
\hline${ }^{214} \mathrm{Po}$ and ${ }^{39} \mathrm{Ar}$ & 2500 \\
\hline
\end{tabular}

Table 5.1: Number of $\alpha$ decays generated. This is used to generate 7 independent components for the fit: ${ }^{222} \mathrm{Rn},{ }^{218} \mathrm{Po},\left({ }^{214} \mathrm{Bi}+{ }^{214} \mathrm{Po}\right),{ }^{220} \mathrm{Rn},{ }^{216} \mathrm{Po},{ }^{212} \mathrm{Bi}$, and surface ${ }^{214}$ Po.

The count branch is used to select a specific number of decays or to inform the fitter how many decays were generated in producing the Monte Carlo ntuple.

- Pileup - An integer representing whether there was no ${ }^{39} \mathrm{Ar}$ pileup (0), ${ }^{39} \mathrm{Ar}$ pre-trigger pileup (1), or ${ }^{39} \mathrm{Ar}$ post-trigger pileup (2).

Once these branches have been added to the Monte Carlo files, the Monte Carlo ntuples are combined into a single file that is used as the input for the fitter.

\section{Fitter Overview}

The fitter consists of two main parts - the energy correction and the fitting of the normalizations of the 7 components. The next two sections will discuss these two modes. 


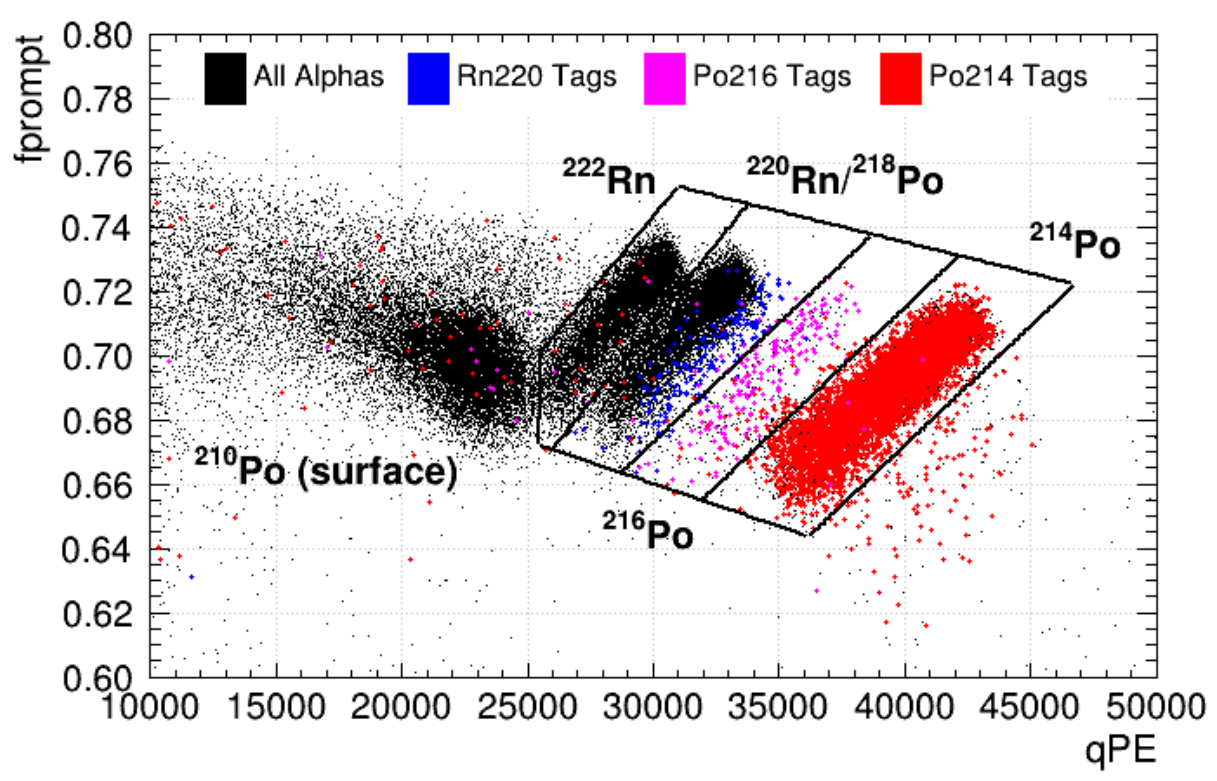

Figure 5.3: The $\alpha$ region in fprompt vs qPE, with the "box cuts" overlaid. The ${ }^{222} \mathrm{Rn},{ }^{218} \mathrm{Po}$, and ${ }^{214} \mathrm{Po}$ peaks dominate in the data. Time coincidence tagging (see Section 5.4) was used to highlight some of the populations. The peak at the far left is surface ${ }^{210} \mathrm{Po}$, which is cut out of the dataset using these cut boundaries before fitting.

\subsubsection{Detailed Description of Energy Correction}

The $\mathrm{qPE}$ or $\mathrm{nSC}$ of the individual $\alpha$ populations overlap. However, in fprompt or rprompt vs $\mathrm{qPE}$ or $\mathrm{nSC}$, it is possible to separate out these populations. Cuts were developed to try to separate these populations as much as possible by rotating the fprompt vs qPE coordinate system until Gaussian peaks were observed and then drawing a line along the minimum. These "box cuts" were saved in a text file for use in various $\alpha$ analyses (cuts shown in Figure 5.3. See section A for explicit cuts). The fitter loads these cuts from this text file and applies them to the data when making its energy correction. The standard pileup and low level cuts (see Appendix A) are also applied to the data and the standard pileup cuts are applied to the Monte Carlo. The cuts used by the fit are described in Section A.

This energy correction uses the fact that the amount of qPE lost is small compared 
to the total $\mathrm{qPE}$ of the event. The equations used to calculate fprompt and $\mathrm{qPE}$ are:

$$
\begin{gathered}
F=\frac{P}{P+L} \\
q=P+L,
\end{gathered}
$$

where $F$ is fprompt, $q$ is qPE, $P$ is the amount of prompt PE, and $\mathrm{L}$ is the amount of late $\mathrm{PE}$. The shift in $\mathrm{qPE}$ and fprompt is primarily due to the digitizer clipping, which dominates over PMT non-linearity. This clipping occurs almost exclusively in the prompt window. Combining the two equations in eq. 5.1 by eliminating the prompt PE (P), we get:

$$
F=\frac{q-L}{q}
$$

In the context of performing an energy correction, we are interested in relating the change in fprompt and qPE due to the digitizer clipping. The change in fprompt is:

$$
\Delta F=\frac{q_{f}-L}{q_{f}}-\frac{q_{i}-L}{q_{i}} \approx \frac{\Delta q}{q}
$$

where the final step is based on the assumption that the amount of clipping is small compared to the total qPE of the event, allowing us to set the denominators in the above equation to be approximately equal to each other. This is a linear relation, and forms the basis of the energy correction.

The initial step is to fit a line to each of the individual $\alpha$ decay "peaks" when plotted in fprompt vs qPE. Each of these lines would represent a single $\alpha$ decay energy. As will be shown, the lines themselves will have decreasing slope with increasing $\alpha$ energy. By making these lines into a continuous energy correction, the digitizer clipping and PMT non-linearity can be corrected. 

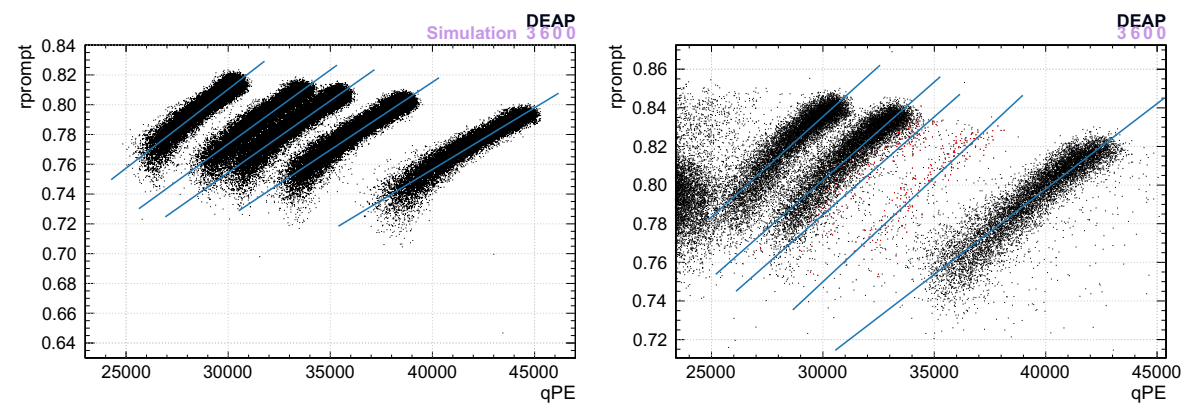

Figure 5.4: Left: Lines fit to the Monte Carlo $\alpha$ populations to correct the effects of digitizer clipping. Right: Lines fit to the $\alpha$ populations in data to correct the effects of digitizer clipping. The black points are (mostly) the ${ }^{222} \mathrm{Rn},{ }^{218} \mathrm{Po}$, and ${ }^{214} \mathrm{Po}$ events, while the red points are tagged ${ }^{220} \mathrm{Rn}$ and ${ }^{216} \mathrm{Po}$. The fit parameter values can be seen in Figures 5.5 and 5.6 . These fits used a $\chi^{2}$ minimization.

As a precaution to prevent any issues arising from float precision, the fprompt and $\mathrm{qPE}$ axes are set to range from 0 to 1 in the $\alpha$ region by doing:

$$
\frac{\text { var }-l \text { Bound }}{u \text { Bound }+ \text { lBound }}
$$

For Monte Carlo events, the "alpha" and "pileup" branches are used to select nonpileup $\alpha$ events for a single $\alpha$ population. A line is then fitted using a $\chi^{2}$ minimization to the fprompt vs qPE distribution of that population, which is approximately linear (see eq. 5.3). This is repeated for each of the $5 \alpha$ populations $\left({ }^{222} \mathrm{Rn},{ }^{220} \mathrm{Rn},{ }^{218} \mathrm{Po}\right.$, ${ }^{216} \mathrm{Po},{ }^{214} \mathrm{Po}$ ) that can be directly identified in data (Figure 5.4, left).

A similar procedure is used in the data. Along with the standard pileup cuts, the "box cuts" are used to select ${ }^{222} \mathrm{Rn},{ }^{218} \mathrm{Po}$, and ${ }^{214} \mathrm{Po}$, all of which have relatively high event counts (around 10,000 each).

${ }^{220} \mathrm{Rn}$ and ${ }^{216} \mathrm{Po}$ are selected using an event list made using $\alpha$ time coincidence tagging, exploiting the 0.145 second half life of ${ }^{216} \mathrm{Po}$, the daughter of ${ }^{220} \mathrm{Rn}$ (see Section 5.4 .3 for more details). The "box cuts" are used to select the regions used for the coincidence tagging.

After fitting the lines in Figure 5.4, the slopes and intercepts of each of the 5 

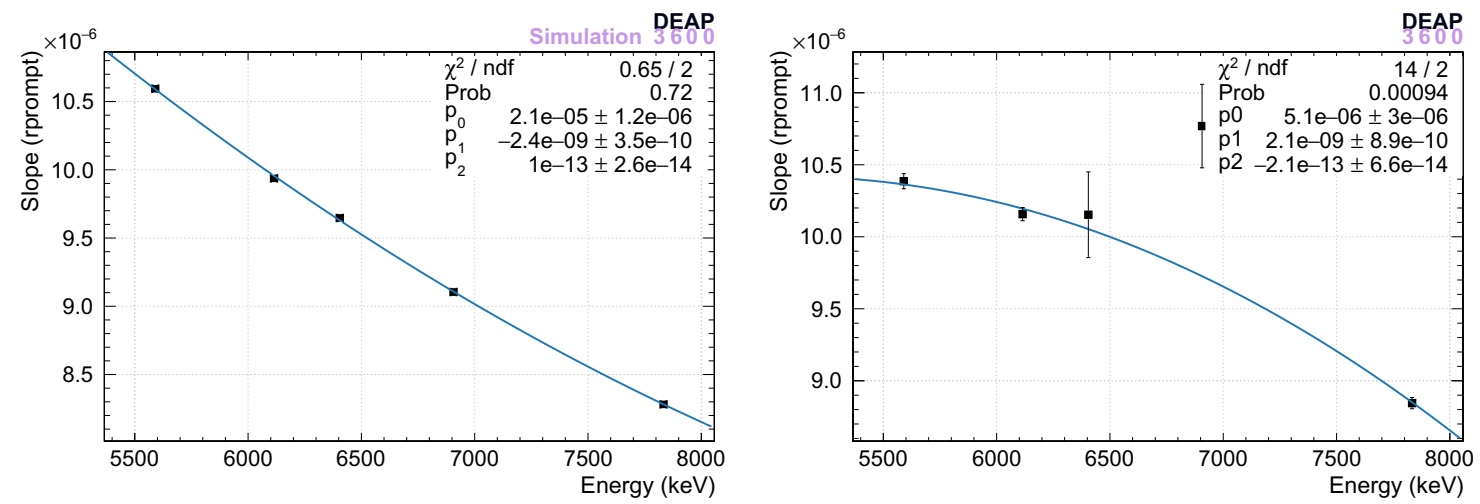

Figure 5.5: Slopes of lines from fits to Monte Carlo (left) and data (right) $\alpha$ distributions in Figure 5.4 as a function of the energy of each distribution. A quadratic is fitted to the 5 points showed here, using a $\chi^{2}$ minimization. The shape of the quadratic function differs between data and MC due to sub-optimal modelling of the detector in the MC. These fits are used in an energy correction that smoothly aligns the data and MC distributions.

Monte Carlo and 5 data fits are extracted. The Monte Carlo and data slopes and intercepts are plotted separately as a function of energy (Figures 5.5 and 5.6). To make these plots, the known energies (in $\mathrm{keV}$ ) of each of the $5 \alpha$ populations is used. A quadratic is fitted to each of the plots, again using a $\chi^{2}$ minimization function. The resulting fit functions form the basis of the energy correction.

In the end, we have 4 curves - slope vs $\mathrm{keV}$ and intercept vs keV for data and for Monte Carlo. So for a given energy, the equation of a line can be obtained. This line is in fprompt vs qPE space. This equation has the form:

$$
\text { fprompt }=q P E \times \operatorname{slope}(k e V)+i n t e r c e p t(k e V) .
$$

Provided this is a one-to-one function of fprompt and qPE, there is only one value of $\mathrm{keV}$ that will satisfy this equation. This equation can be rearranged:

$$
\text { fprompt }-(q P E \times \operatorname{slope}(k e V)+\operatorname{intercept}(k e V))=0 \text {. }
$$



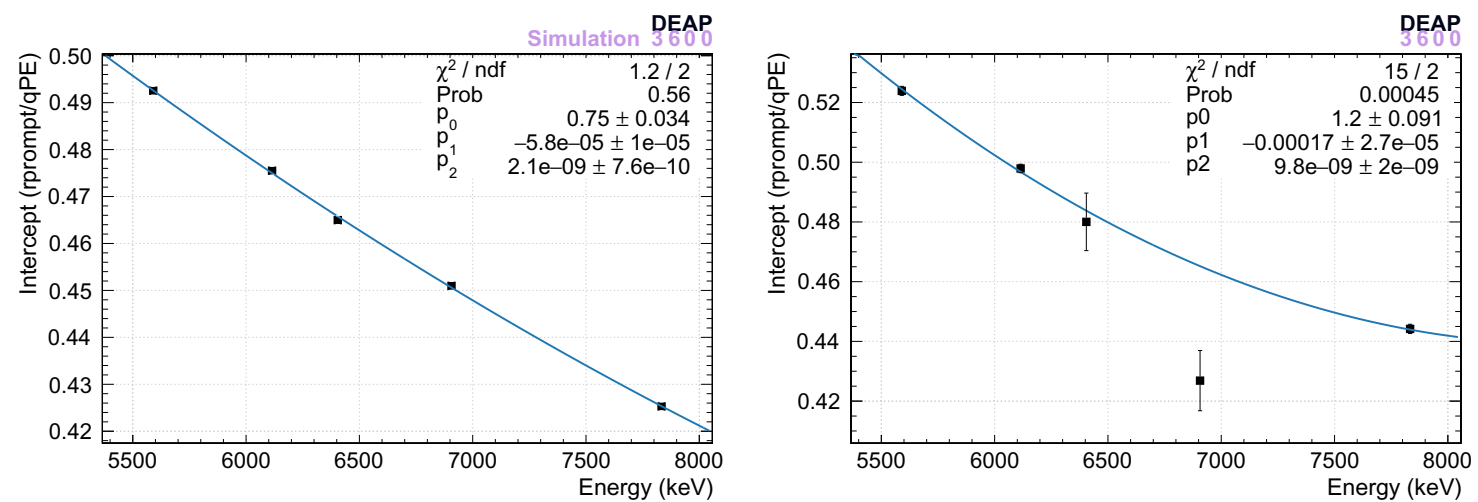

Figure 5.6: Intercepts of lines from fits to Monte Carlo (left) and data (right) $\alpha$ distributions in Figure 5.4 as a function of the energy of each distribution. A quadratic is fitted to the 5 points showed here, using a $\chi^{2}$ minimization. The ${ }^{216} \mathrm{Po}$ fit to the data is an outlier, and this is thought to be due to random coincidence tags may be present in the ${ }^{216} \mathrm{Po}$ sample. The shape of the quadratic function differs between data and $\mathrm{MC}$ due to sub-optimal modelling of the detector in the MC. These fits are used in an energy correction that smoothly aligns the data and $\mathrm{MC}$ distributions.

ROOT's "GetMinimumX" method in the "TF1" class is used to minimize the left hand side of this equation for a given pair of fprompt and qPE. Thus, we have a conversion from fprompt and qPE to keV for both data and Monte Carlo.

\subsubsection{Detailed description of fitting methods}

Once the energy correction has been performed on the Monte Carlo and data events, the fitter proceeds with the fitting of the normalizations of the individual $\alpha$ decay populations (the fit "components"). Before starting this fit, the normalization of the ${ }^{39}$ Ar pileup contribution to each fit component is scaled through the use of the "count" branch described above to match the statistical expectation for each population.

This fit has 8 parameters - the 7 component normalizations and an overall energy resolution term. These components are used to adjust the Monte Carlo energy spectrum. No adjustment is made to the data spectrum.

The fitter starts by getting a best fit of the 7 normalizations by minimizing the $\chi^{2}$ 
using Minuit, and then proceeds to run a grid search for the resolution term. It then does a binned log-likelihood fit of the 7 normalizations. It repeats the grid search for the resolution term, and then performs a final binned log-likelihood fit of the 7 normalizations plus the resolution term.

The normalizations and total decays generated in the Monte Carlo are used to output a final estimated number of decays for each $\alpha$ population in the dataset. The resulting fit is shown in Figure 5.7 .

\section{Resolution term}

The resolution term required the development of an algorithm capable of broadening the individual $\alpha$ peak widths without requiring computationally expensive re-binning

of the underlying histograms. In addition to the computation time involved with rebinning, the fact that the effect of re-binning is discreet (an integer number of events move from one bin to another), there exists the possibility that a small change in the resolution will not effect any change in the weights of the bins in the MC histogram, which would cause the ROOT fitting algorithms to fail. There are also many local minima in this fit, so Minuit will not converge on the correct minimum unless the resolution term is already close to the best value.

These issues were avoided by stretching or compressing the component histograms along the energy axis through the use of a constant "stretching" factor, which is proportional to the resolution term. This allows Minuit to find continuous derivatives for the resolution term at any scale.

The fit function references the components (created from the individual $\alpha \mathrm{MC}$ isotopes) by summing them binwise and multiplying by the normalization for each 
value of $x$ requested by the fitter:

$$
f(x)=\sum_{i=1}^{7} C_{i}(x) \cdot n_{i}
$$

where $C_{i}(x)$ is the raw event count in the bin corresponding to $x$ for the $i$ th component and $n_{i}$ are the normalizations for the $i$ th component.

In order to implement the resolution factor, the bin from each component that is referenced by the fit function is shifted slightly:

$$
f(x)=\sum_{i=1}^{7} \frac{C_{i}\left(\left(x-E_{i}\right) / R+E_{i}\right) \cdot n_{i}}{R},
$$

where $E$ are the known energies of the $\alpha$ decay and $R$ is the resolution factor. Note that the division by $R$ preserves the integral of the MC histograms. Note that this equation does not attempt to model the resolution, but rather attempts to correct the mismatch between the predictions of the Monte Carlo and the observations in the data. If the constant (in energy) scaling of the simulated resolution had proven insufficient, a more complex resolution correction would have been implemented.

However, one bi-product of this smooth adjustment of the component histograms is that the bins of the MC histogram and data histogram would in general no longer be aligned. The $\chi^{2}$ test performs one evaluation of the residual for each bin in the data histogram, which requires that the histogram of the fit function be aligned with that of the data. This misalignment was corrected in the fit function as follows in the next paragraph.

When a value is requested for a given $x$, the fit function starts by getting the upper and lower bounds $\left(x_{l o w}\right.$ and $\left.x_{u p}\right)$ of the bin in the data histogram containing this $\mathrm{x}$ value. It then gets the $\mathrm{MC}$ bin numbers for each component at these 2 bounds, 
according to equation 5.9 .

$$
x^{\prime}=\left(x-E_{i}\right) / R+E_{i} .
$$

If the MC bin numbers at $x_{l o w}^{\prime}$ and $x_{u p}^{\prime}$ are the different, the fit function adds them to the sum, but weights them according to the fraction of each bin that overlaps with the data bin. Any bins that exist between these two MC bins are also added to the sum. Finally, the sum is divided by the resolution multiplicative scaling factor to obtain an average value for the component over the range of the data bin. This process is summarized below:

1. Get upper and lower bound of data histogram bin $\left(x_{\text {low }}\right.$ and $\left.x_{u p}\right)$

2. Calculate $x_{\text {low }}^{\prime}$ and $x_{\text {up }}^{\prime}$ using eq. 5.9

3. Take the weighted average of bin values, being careful to preserve the integral number of events

The challenge of the fit converging on a local minima was overcome by using a grid search to get the resolution term close to the best fit value before performing the fit described above. The fitter alternated between using Minuit to fit the 7 normalizations, and performing the grid search on the resolution term.

\section{Fit Bounds}

The upper fit bound of $9000 \mathrm{keV}$ was chosen to allow some of the ${ }^{214}$ Po pileup tail to be included in the fit without including significant numbers of ${ }^{212}$ Po events (see Section 5.3 for a discussion of these). The lower fit bound of $5400 \mathrm{keV}$ was chosen to exclude significant leakage from the ${ }^{210} \mathrm{Po}$ distribution. This cut removed part of the lower energy tail of the ${ }^{222} \mathrm{Rn}$ distribution (see Figure 5.3 ), resulting in a deficit below $5400 \mathrm{keV}$. 

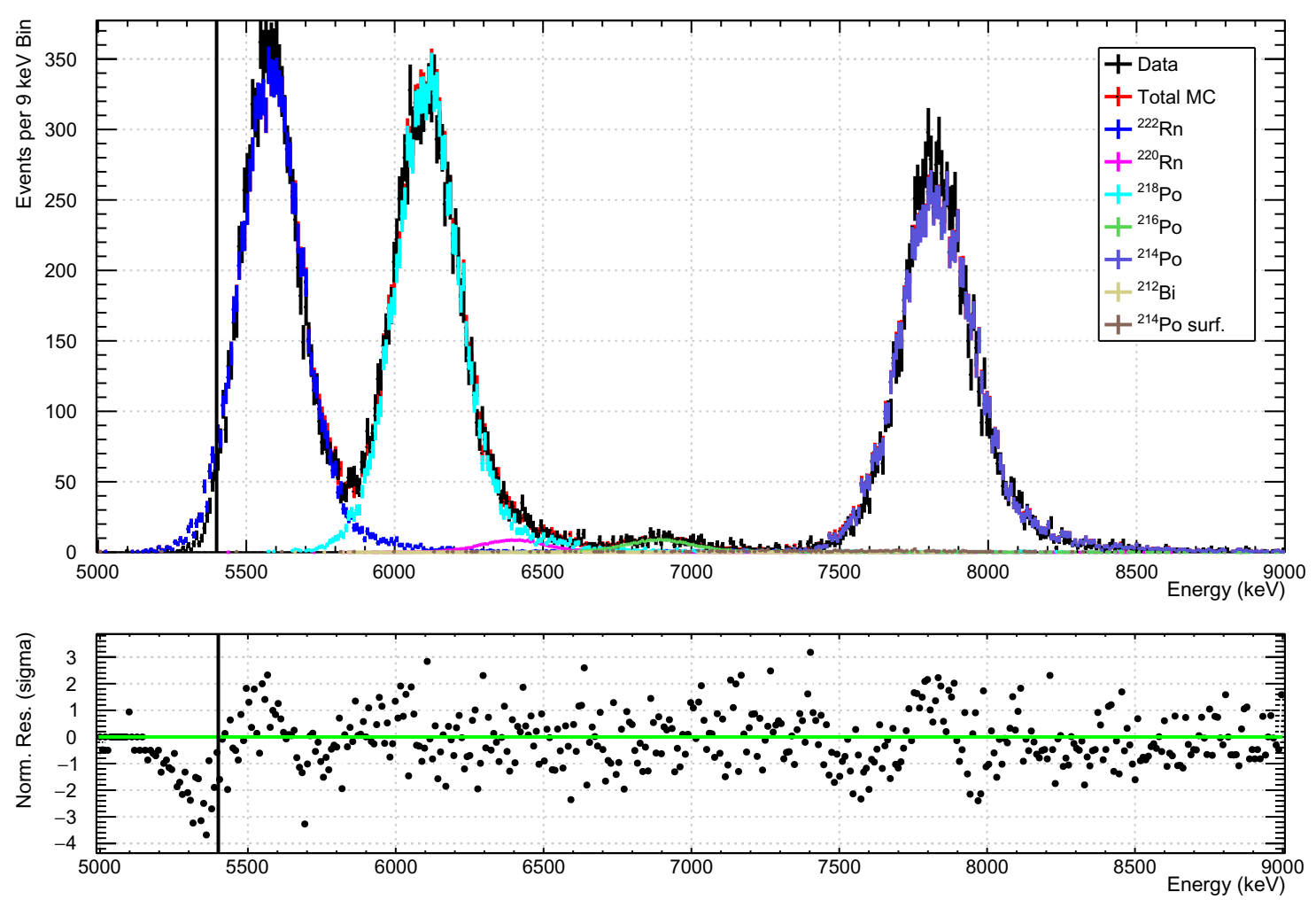

Figure 5.7: Above: Best fit of the corrected Monte Carlo energy spectrum to the corrected $\alpha$ data energy spectrum. Below: The residuals of the above fit. The vertical black line is the lower fit boundary. The region to the left of the line was excluded due to the removal of ${ }^{222} \mathrm{Rn}$ events in data by the ${ }^{210} \mathrm{Po}$ exclusion cut. The following values are for the region to the right of the line: $\chi^{2} / \mathrm{NDF}=$ $1.09, \chi^{2}=426$, Prob $=0.151, \mathrm{NDF}=390$.

\subsubsection{Results}

The numerical result of the fit is listed in table 5.2, while the resulting spectrum is shown in Figure 5.7. Note that although the $\chi^{2}$ is quoted in the caption, the fit was done using a binned log-likelihood. 

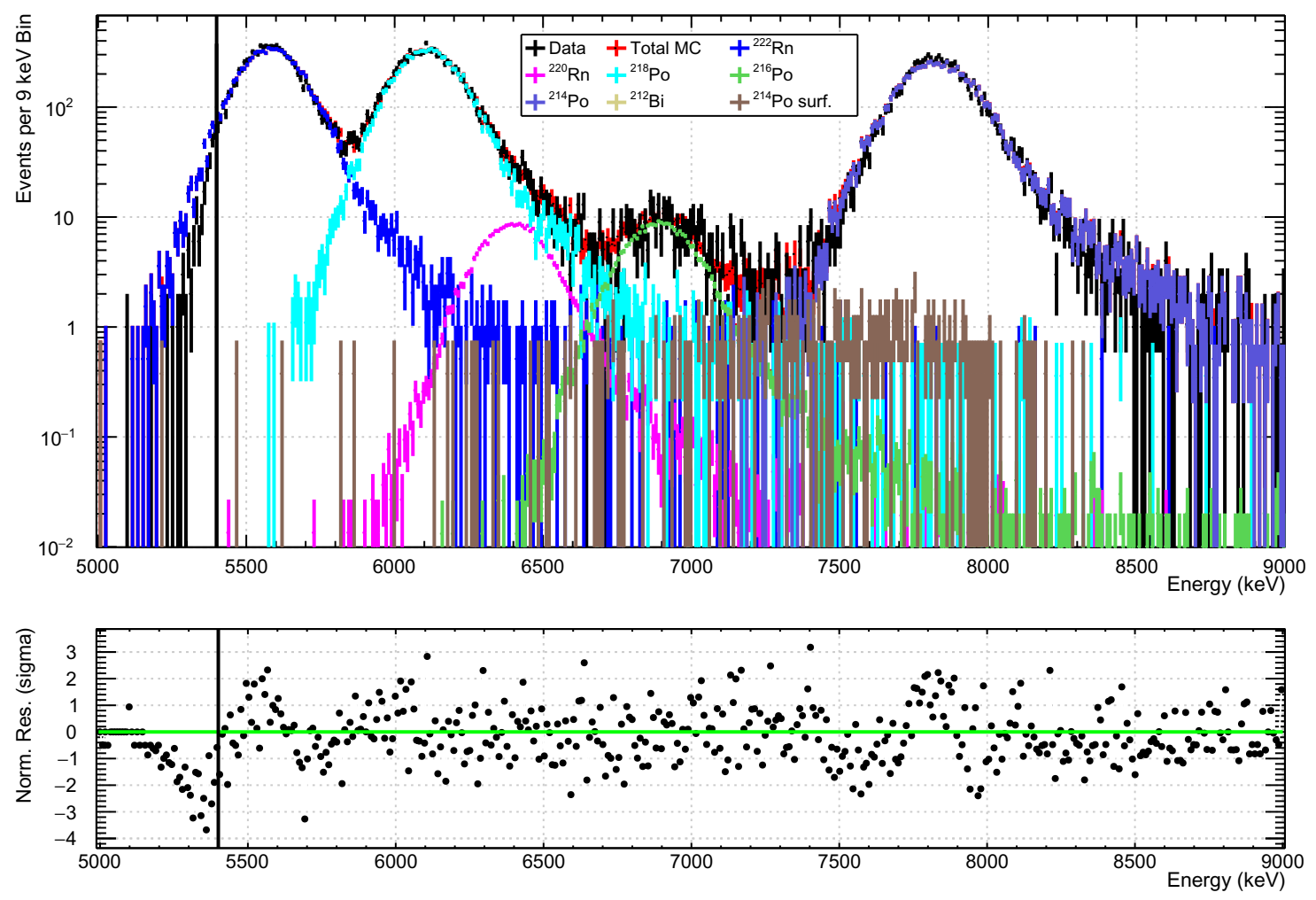

Figure 5.8: Figure 5.7 in log scale. 


\begin{tabular}{|c|c|c|c|c|c|c|c|}
\hline & ${ }^{222} \mathbf{R n}$ & ${ }^{220} \mathbf{R n}$ & ${ }^{218} \mathbf{P o}$ & ${ }^{216} \mathbf{P o}$ & ${ }^{214} \mathbf{P o}$ & ${ }^{212} \mathbf{B i}$ & Surf. ${ }^{214} \mathbf{P o}$ \\
\hline${ }^{222} \mathbf{R n}$ & 1 & 0.0018897 & -0.036276 & -0.0047485 & 0.00059043 & $7.5082 \mathrm{e}-05$ & -0.0068758 \\
${ }^{220} \mathbf{R n}$ & 0.0018897 & 1 & -0.25121 & -0.054654 & 0.005305 & -0.0040131 & -0.037051 \\
${ }^{218} \mathbf{P o}$ & -0.036276 & -0.25121 & 1 & -0.007589 & 0.00096575 & -0.0028394 & -0.014246 \\
${ }^{216} \mathbf{P o}$ & -0.0047485 & -0.054654 & -0.007589 & 1 & 0.038615 & $1.0329 \mathrm{e}-05$ & -0.26197 \\
${ }^{214} \mathbf{P o}$ & 0.00059043 & 0.005305 & 0.00096575 & 0.038615 & 1 & $2.0658 \mathrm{e}-05$ & -0.16639 \\
${ }^{212} \mathbf{B i}$ & $7.5082 \mathrm{e}-05$ & -0.0040131 & -0.0028394 & $1.0329 \mathrm{e}-05$ & $2.0658 \mathrm{e}-05$ & 1 & -0.00026802 \\
Surf. ${ }^{214} \mathbf{P o}$ & -0.0068758 & -0.037051 & -0.014246 & -0.26197 & -0.16639 & -0.00026802 & 1 \\
\hline
\end{tabular}

Table 5.3: Correlation matrix for fit in Figure 5.7 .

\begin{tabular}{|c|c|c|}
\hline Isotope & Activity (events/247.17 days) & Specific Activity $(\mu \mathrm{Bq} / \mathrm{kg})$ \\
\hline${ }^{222} \mathrm{Rn}$ & $\begin{array}{l}10740_{-110(\text { stat })-80(\text { syst })}^{+110(\text { stat })+(\text { syt })} \\
\end{array}$ & $\begin{array}{l}0.1534_{-0.0048(\text { stat })-0.0012(\text { syst })}^{+0.0048(\text { stat })} \\
\end{array}$ \\
\hline${ }^{220} \mathrm{Rn}$ & $299_{-60(\text { stat })-62(\text { syst })}^{+40(\text { stat })+17 \text { sys }}$ & 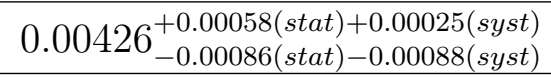 \\
\hline${ }^{218} \mathrm{Po}$ & $11160_{-150(\text { stat })-590(\text { syst })}^{+120(\text { stat })+20(\text { st })}$ & $0.1593_{-0.0051(\text { stat })-0.0084(\text { syst })}^{+0.0050}$ \\
\hline${ }^{216} \mathrm{Po}$ & $\begin{array}{l}312.493_{-23(\text { stat })-32(\text { syst })}^{+23(\text { stat })} \\
\end{array}$ & $\begin{array}{l}0.00446 \\
0.00036(\text { stat })+0.00004(\text { syst }) \\
\end{array}$ \\
\hline${ }^{214} \mathrm{Po}$ & $10740_{-110(\text { stat })-150(\text { syst })}^{+110(\text { stat })}$ & $0.1534_{-0.0048(\text { stat })-0.0022(\text { syst })}^{+0.0048(\text { stat })+0.0004 \text { (syst }}$ \\
\hline${ }^{212} \mathrm{Bi}$ & $0_{-0}^{+300(\text { stat })+2200(\text { syst })}$ & $0_{-0}^{+0.004(\text { stat })+0.031(\text { syst })}$ \\
\hline${ }^{214}$ Po surf. & \multicolumn{2}{|c|}{$133_{-34(\text { stat })-21(\text { syst })}^{+40(\text { stat })+20(\text { syst }}$ events in fit range } \\
\hline
\end{tabular}

Table 5.2: LAr bulk activities for $\mathrm{U}$ and Th chain isotopes.

Note that this activity estimate assumes that the trigger efficiency in the Monte Carlo is a good representation of the true trigger efficiency in the detector. The statistical uncertainties here are from the ROOT Minuit fit and the systematic uncertainty includes uncertainty within the MC model and in the energy correction, as estimated from the systematic study detailed in section 5.2.6. The results with the systematic and statistical uncertainties added in quadrature are shown in table 5.4 .

For reference, table 5.5 lists the number of triggers in the dataset, obtained through the "box cuts". 


\begin{tabular}{|c|c|c|}
\hline Isotope & Activity (events $/ \mathbf{2 4 7 . 1 7}$ days) & Specific Activity $(\mu \mathbf{B q} / \mathbf{k g})$ \\
\hline${ }^{222} \mathrm{Rn}$ & $10740_{-140}^{+110}$ & $0.1534_{-0.0049}^{+0.0048}$ \\
\hline${ }^{220} \mathrm{Rn}$ & $299_{-86}^{+43}$ & $0.0043_{-0.0012}^{+0.0006}$ \\
\hline${ }^{218} \mathrm{Po}$ & $11160_{-610}^{+120}$ & $0.1593_{-0.0098}^{+0.0050}$ \\
\hline${ }^{216} \mathrm{Po}$ & $312_{-39}^{+23}$ & $0.00446_{-0}^{+0.000036}$ \\
\hline${ }^{214} \mathrm{Po}$ & $10740_{-190}^{+110}$ & $0.1534_{-0.0053}^{+0.0048}$ \\
\hline${ }^{212} \mathrm{Bi}$ & $0_{-0}^{+2200}$ & $0_{-0}^{+0.031}$ \\
\hline${ }^{214} \mathrm{Po}$ surf. & \multicolumn{2}{|c|}{$130_{-40}^{+200}$ events in fit range } \\
\hline
\end{tabular}

Table 5.4: LAr bulk activities for $\mathrm{U}$ and Th chain isotopes with systematic errors added to the statistical errors in table 5.2 .

\begin{tabular}{|c|c|}
\hline Isotope & Number of Events in Box (247.17 days) \\
\hline${ }^{222} \mathrm{Rn}$ & 10904 \\
\hline${ }^{218} \mathrm{Po}$ & 10640 \\
\hline${ }^{214} \mathrm{Po}$ & 10493 \\
\hline${ }^{220} \mathrm{Rn}$ & $264\left(\right.$ time tagged with $\left.{ }^{216} \mathrm{Po}\right)$ \\
\hline${ }^{216} \mathrm{Po}$ & $264\left(\right.$ time tagged with $\left.{ }^{220} \mathrm{Rn}\right)$ \\
\hline
\end{tabular}

Table 5.5: Number of events in "box cut" regions. Note that the last two entries required time tagging to identify due to low statistics and their proximity to the other $\alpha$ distributions. 


\subsubsection{Systematics Study}

In an attempt to better understand the systematic uncertainties in the fit, 5 parameters were set to extreme values (typically over-estimates) and the fit was rerun. The fits are shown in Appendix B. The systematic shifts are listed below:

- MC slopes in Figure 5.5 used to calibrate energy correction - varied within their uncertainties. See Figures B.1 and B.2 and Tables B.1 and B.3.

- $\mathrm{MC}$ intercepts in Figure 5.6 used to calibrate energy correction - varied within their uncertainties. See Figures B.3 and B.4 and Tables B.5 and B.7.

- $\mathrm{qPE}$ of MC events - shifted up and down by $0.2 \%$ after calibrating the energy correction but before applying it to events. See Figures B.5 and B.6 and Tables B.9 and B.11.

- fprompt of $\mathrm{MC}$ events - shifted up and down by $0.2 \%$ after calibrating the energy correction but before applying it to events. See Figures B.7 and B.8 and Tables B.13 and B.15.

- Resolution term - varied within estimated uncertainties. See Figures B.9 and B.10 and Tables B.17 and B.19.

Although a $0.2 \%$ shift may seem small, the fits involving post-calibration shifts to fprompt and qPE were not used in assigning systematic uncertainties, as miscalibration of the energy correction were already accounted for by the energy calibration uncertainties. In addition, the fits after performing these particular variations were quite poor, making it clear that they were in fact over-estimates and that they would not yield good estimates of systematic uncertainty.

The uncertainties for the tests listed above were assigned by the ROOT fit. When applying variations to the slopes or intercepts, for example, the slopes or intercepts 
were all shifted up or down at the same time. Since these tests are expected to produce over-estimates of the systematic uncertainty, the systematic errors were assigned based on the largest of the errors (bolded) in tables $5.6-5.11$ 


\section{Assignment of Error Values}

The tables below show the shift in results of the fit after performing a systematic variation. The values are calculated by taking the difference between the best fit values for each parameter and the values returned by the fit after performing a systematic variation. A positive "Delta Activity" indicates that the value returned after the systematic variation was larger than the best fit value, while a negative "Delta Activity" indicates the opposite.

The largest positive and negative deviations from the best fit value for each parameter are assigned as the upper and lower systematic uncertainty for that parameter. These values are bolded in tables 5.6-5.11.

\begin{tabular}{|c|c|c|}
\hline Isotope & $\begin{array}{c}\text { Delta Activity } \\
(\text { events } / \mathbf{2 4 7 . 1 7} \text { days })\end{array}$ & $\begin{array}{c}\text { Delta Specific } \\
\text { Activity }(\mu \mathrm{Bq} / \mathbf{k g})\end{array}$ \\
\hline${ }^{222} \mathrm{Rn}$ & $\mathbf{- 8 3 . 0 9 2 6}$ & $\mathbf{- 0 . 0 0 1 1 8 6 6 2}$ \\
\hline${ }^{220} \mathrm{Rn}$ & -21.2899 & -0.000304035 \\
\hline${ }^{218} \mathrm{Po}$ & -68.2901 & -0.000975229 \\
\hline${ }^{216} \mathrm{Po}$ & $\mathbf{- 3 1 . 5 0 3 2}$ & $\mathbf{- 0 . 0 0 0 4 4 9 8 8 8}$ \\
\hline${ }^{214} \mathrm{Po}$ & $\mathbf{- 1 5 2 . 8 6 5}$ & $\mathbf{- 0 . 0 0 2 1 8 3 0 2}$ \\
\hline${ }^{212} \mathrm{Bi}$ & $-5.08344 \mathrm{e}-05$ & $-7.2595 \mathrm{e}-10$ \\
\hline${ }^{214} \mathrm{Po}$ surf. & $\mathbf{1 9 8 . 6 8 3}$ events in fit range \\
\hline
\end{tabular}

Table 5.6: Fit result when MC slopes in Figure 5.5 are shifted up by 1 sigma. See Figure B.1. $\chi^{2} / \mathrm{NDF}=1.62$ 


\begin{tabular}{|c|c|c|}
\hline Isotope & $\begin{array}{c}\text { Delta Activity } \\
\text { (events } / \mathbf{2 4 7 . 1 7} \text { days })\end{array}$ & $\begin{array}{c}\text { Delta Specific } \\
\text { Activity }(\mu \mathrm{Bq} / \mathbf{k g})\end{array}$ \\
\hline${ }^{222} \mathrm{Rn}$ & -46.3413 & -0.000661786 \\
\hline${ }^{220} \mathrm{Rn}$ & -29.1479 & -0.000416252 \\
\hline${ }^{218} \mathrm{Po}$ & $\mathbf{- 5 8 8 . 5 0 4}$ & $\mathbf{- 0 . 0 0 8 4 0 4 2 5}$ \\
\hline${ }^{216} \mathrm{Po}$ & -1.2837 & $-1.83321 \mathrm{e}-05$ \\
\hline${ }^{214} \mathrm{Po}$ & $\mathbf{2 7 . 1 6 2 2}$ & $\mathbf{0 . 0 0 0 3 8 7 8 9 5}$ \\
\hline${ }^{212} \mathrm{Bi}$ & $\mathbf{2 1 8 1 . 7 7}$ & $\mathbf{0 . 0 3 1 1 5 7 2}$ \\
\hline${ }^{214} \mathrm{Po} \mathrm{surf.}$ & $\mathbf{- 2 0 . 5 7 7 7}$ events in fit range \\
\hline
\end{tabular}

Table 5.7: Fit result when MC slopes in Figure 5.5 are shifted down by 1 sigma. See Figure B.2. $\chi^{2} / \mathrm{NDF}=1.53$

\begin{tabular}{|c|c|c|}
\hline Isotope & $\begin{array}{c}\text { Delta Activity } \\
(\text { events } / \mathbf{2 4 7 . 1 7} \text { days })\end{array}$ & $\begin{array}{c}\text { Delta Specific } \\
\text { Activity }(\mu \mathbf{B q} / \mathbf{k g})\end{array}$ \\
\hline${ }^{222} \mathrm{Rn}$ & -0.426286 & $-6.08765 \mathrm{e}-06$ \\
\hline${ }^{220} \mathrm{Rn}$ & $\mathbf{- 6 1 . 8 7 8 4}$ & $\mathbf{- 0 . 0 0 0 8 8 3 6 6 5}$ \\
\hline${ }^{218} \mathrm{Po}$ & 23.6435 & 0.000337646 \\
\hline${ }^{216} \mathrm{Po}$ & -6.01185 & $-8.58534 \mathrm{e}-05$ \\
\hline${ }^{214} \mathrm{Po}$ & -20.9827 & -0.000299647 \\
\hline${ }^{212} \mathrm{Bi}$ & 0.00024505 & $3.49949 \mathrm{e}-09$ \\
\hline${ }^{214} \mathrm{Po}$ surf. & 56.0668 events in fit range \\
\hline
\end{tabular}

Table 5.8: Fit result when MC intercepts in Figure 5.6 are shifted up by 1 sigma. See Figure B.3. $\chi^{2} / \mathrm{NDF}=1.36$ 


\begin{tabular}{|c|c|c|}
\hline Isotope & $\begin{array}{c}\text { Delta Activity } \\
\text { (events } / \mathbf{2 4 7 . 1 7} \text { days })\end{array}$ & $\begin{array}{c}\text { Delta Specific } \\
\text { Activity }(\mu \mathbf{B q} / \mathbf{k g})\end{array}$ \\
\hline${ }^{222} \mathrm{Rn}$ & -20.521 & -0.000293054 \\
\hline${ }^{220} \mathrm{Rn}$ & -21.5554 & -0.000307827 \\
\hline${ }^{218} \mathrm{Po}$ & -412.98 & -0.00589764 \\
\hline${ }^{216} \mathrm{Po}$ & -6.71831 & $-9.59421 \mathrm{e}-05$ \\
\hline${ }^{214} \mathrm{Po}$ & 16.5438 & 0.000236257 \\
\hline${ }^{212} \mathrm{Bi}$ & 1601.57 & 0.0228715 \\
\hline${ }^{214} \mathrm{Po}$ surf. & \multicolumn{2}{|c|}{8.51663 events in fit range } \\
\hline
\end{tabular}

Table 5.9: Fit result when $\mathrm{MC}$ intercepts in Figure 5.6 are shifted down by 1 sigma. See Figure B.4. $\chi^{2} / \mathrm{NDF}=1.18$

\begin{tabular}{|c|c|c|}
\hline Isotope & $\begin{array}{c}\text { Delta Activity } \\
\text { (events } / \mathbf{2 4 7 . 1 7} \text { days })\end{array}$ & $\begin{array}{c}\text { Delta Specific } \\
\text { Activity }(\mu \mathrm{Bq} / \mathbf{k g})\end{array}$ \\
\hline${ }^{222} \mathrm{Rn}$ & $\mathbf{3 . 4 8 0 5 9}$ & $\mathbf{4 . 9 7 0 5 2 e - 0 5}$ \\
\hline${ }^{220} \mathrm{Rn}$ & $\mathbf{1 7 . 2 5 4 4}$ & $\mathbf{0 . 0 0 0 2 4 6 4 0 4}$ \\
\hline${ }^{218} \mathrm{Po}$ & $\mathbf{2 4 . 6 2 5 4}$ & $\mathbf{0 . 0 0 0 3 5 1 6 6 7}$ \\
\hline${ }^{216} \mathrm{Po}$ & 0.430546 & $6.14849 \mathrm{e}-06$ \\
\hline${ }^{214} \mathrm{Po}$ & 5.3423 & $7.62917 \mathrm{e}-05$ \\
\hline${ }^{212} \mathrm{Bi}$ & 4.67096 & $6.67045 \mathrm{e}-05$ \\
\hline${ }^{214} \mathrm{Po}$ surf. & \multicolumn{2}{|c|}{-2.31087 events in fit range } \\
\hline
\end{tabular}

Table 5.10: Fit result when resolution term is shifted up by 1 sigma and normalizations are refitted. See Figure B.9. $\chi^{2} / \mathrm{NDF}=1.11$ 


\begin{tabular}{|c|c|c|}
\hline Isotope & $\begin{array}{c}\text { Delta Activity } \\
\text { (events } / \mathbf{2 4 7 . 1 7} \text { days })\end{array}$ & $\begin{array}{c}\text { Delta Specific } \\
\text { Activity }(\mu \mathbf{B q} / \mathbf{k g})\end{array}$ \\
\hline${ }^{222} \mathrm{Rn}$ & 1.72565 & $2.46434 \mathrm{e}-05$ \\
\hline${ }^{220} \mathrm{Rn}$ & 0.797347 & $1.13867 \mathrm{e}-05$ \\
\hline${ }^{218} \mathrm{Po}$ & 1.33857 & $1.91157 \mathrm{e}-05$ \\
\hline${ }^{216} \mathrm{Po}$ & $\mathbf{2 . 9 0 4 6 2}$ & $\mathbf{4 . 1 4 7 9 9 e - 0 5}$ \\
\hline${ }^{214} \mathrm{Po}$ & 7.69204 & 0.000109848 \\
\hline${ }^{212} \mathrm{Bi}$ & 0.000881666 & $1.25908 \mathrm{e}-08$ \\
\hline${ }^{214} \mathrm{Po}$ surf. & -9.6743 events in fit range \\
\hline
\end{tabular}

Table 5.11: Fit result when resolution term is shifted down by 1 sigma and normalizations are refitted. See Figure B.10, $\chi^{2} / \mathrm{NDF}=1.10$ 


\section{$5.3 \quad{ }^{212} \mathrm{Po}$}

${ }^{212}$ Po energies were not included in the global fit. There were two reasons for this. The first is that PMT non-linearity becomes quite strong very quickly at ${ }^{212}$ Po energies $(8.9 \mathrm{MeV})$, preventing the energy correction method from properly correcting the data without modification to the calibration function. The second reason is that the nonlinear shape of the ${ }^{212} \mathrm{Po}$ distribution in fprompt vs qPE space prevents this peak from acting as its own calibration source, unlike the other $\alpha$ peaks. Since no calibration source was present and extrapolation to high energies was not possible, ${ }^{212} \mathrm{Po}$ had to be excluded from the multi-component fit described in the previous section.

A simple cut and count measurement of ${ }^{212}$ Po candidates in the liquid argon was made instead, and the Monte Carlo was used to convert this to an activity.

\subsubsection{Cuts}

The Monte Carlo predicts ${ }^{212}$ Po to occur in the region of fmaxpeprompt vs qPE_60 space shown in Figure 5.9. There is a slight offset between MC and data due to PMT non-linearity, however, so cuts used to identify ${ }^{212} \mathrm{Po}$ in MC cannot be directly applied to data. The location of the ${ }^{214}$ Po distribution was used to match the data and MC cuts that were used to count ${ }^{212} \mathrm{Po}$.

The choice of selection cut variables was made to optimize the separation of ${ }^{214} \mathrm{Po}$ and ${ }^{212} \mathrm{Po}$ events while using a linear cut. When compared with qPE, qPE_60 is less affected by the residual light of the preceding bismuth decay, particularly for ${ }^{214} \mathrm{Po}$, which results in a more narrow distribution. nSC was much more broad for the $\mathrm{MC}{ }^{212} \mathrm{Po}$ events than the qPE based variables, which excluded nSC as an option. fmaxpeprompt was chosen in the place of fprompt for the same reasons that motivated the choice of qPE_60 over qPE.

Figure 5.10 shows the data region that was used to count the ${ }^{212}$ Po events in the 


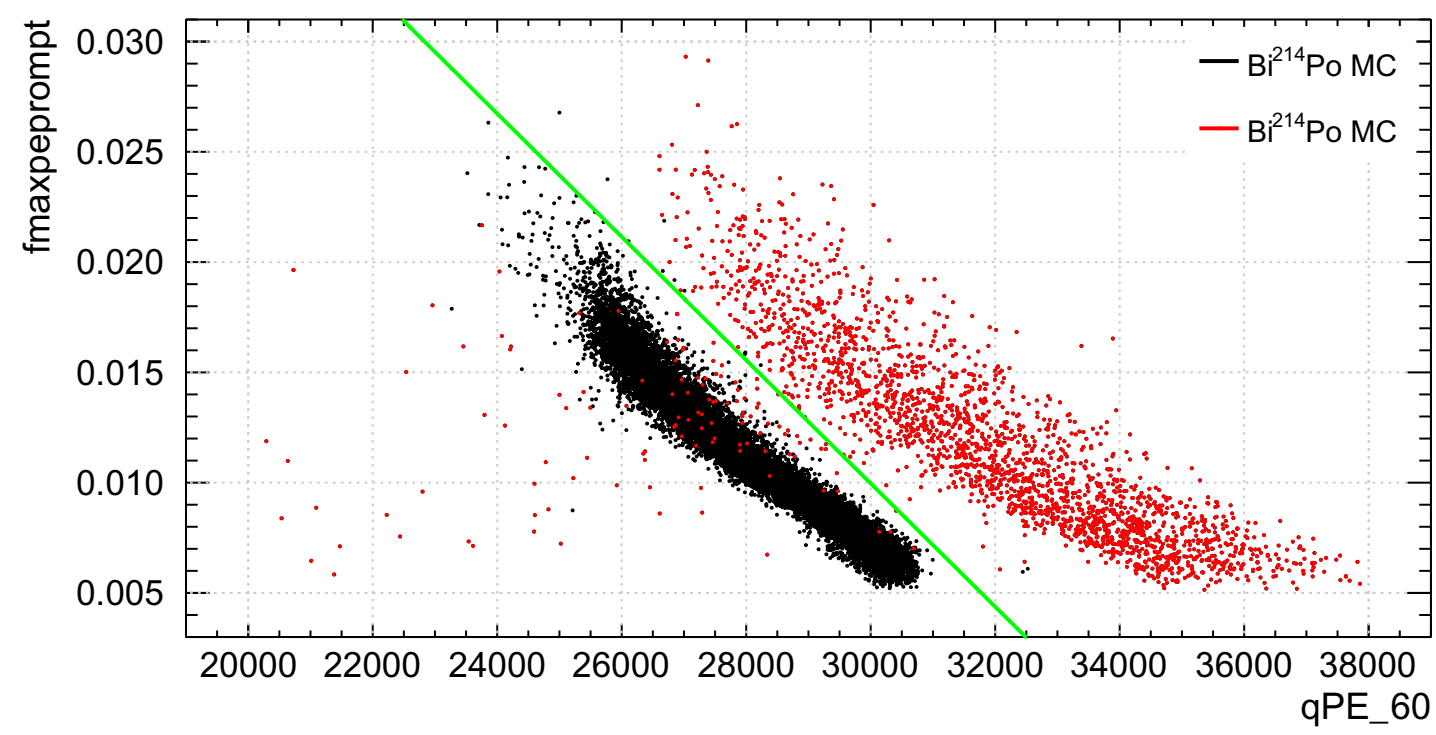

Figure 5.9: Monte Carlo of ${ }^{212} \mathrm{Po}$ and nearby $\alpha$ populations. 20,000 decays were produced of ${ }^{212} \mathrm{Bi}^{208} \mathrm{~Pb}$ and ${ }^{214} \mathrm{Bi}^{2}{ }^{210} \mathrm{~Pb}$, which are both present in this plot. The black distribution is ${ }^{214} \mathrm{Po}$, while the red distribution is ${ }^{212} \mathrm{Po}$. The line represents the equivalent $\mathrm{MC}$ version of the cut that was used to count data events in Figure 5.10 .

data. This region was selected using the Monte Carlo, shown in Figure 5.9. The cuts were tuned to minimize leakage from the dominant ${ }^{214} \mathrm{Po}$ distribution.

The MC selection cuts used are:

- $\mathrm{qPE}>40000$

- $\mathrm{qPE} \_60>20000$

- fmaxpeprompt $>$ qPE_60*-2.79497e-06 + 0.0938144

The data selection cuts are:

- Bad trigger cuts

- $40000<\mathrm{qPE}<70000$

- $\mathrm{qPE} \_60>22000$ 


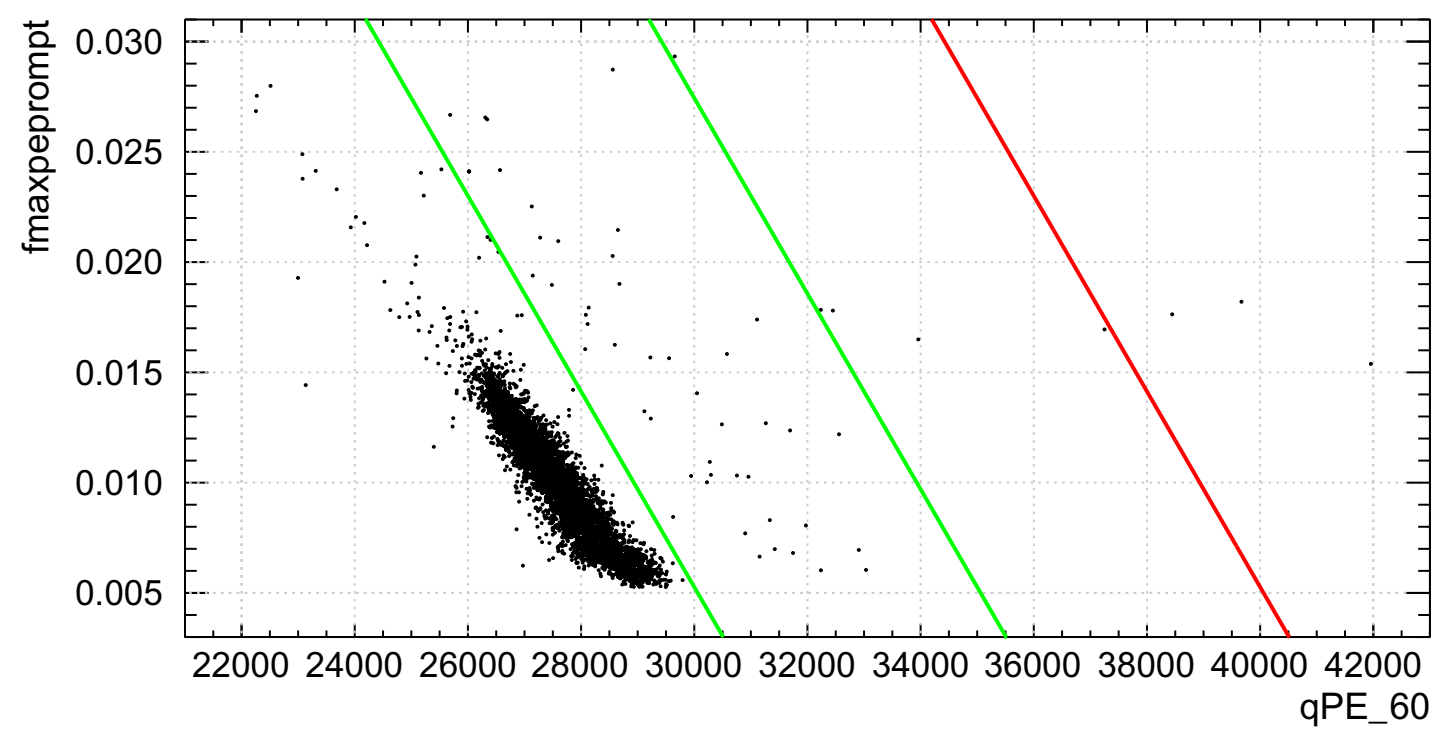

Figure 5.10: Data events in the ${ }^{214} \mathrm{Po}$ and ${ }^{212} \mathrm{Po}$ region. The dominant distribution is ${ }^{214} \mathrm{Po}$. The ${ }^{212} \mathrm{Po}$ events are expected to be in the region between the green lines. The region between the green and red line was used to estimate the background, as no $\mathrm{MC}$ events were present in the corresponding $\mathrm{MC}$ region (see Figure 5.9.

- fmaxpeprompt > qPE_60*-4.43416e-06 + 0.138292

- fmaxpeprompt $<$ qPE_60*-4.43416e-06 + 0.160463

- fmaxpeprompt $<0.03$

\subsubsection{Result}

27 data events were counted in the region shown in Figure 5.10, $1840 \mathrm{MC}$ events were counted in the region shown in Figure 5.9. 20,000 ${ }^{212} \mathrm{Bi}$ decays were generated to produce these 1840 events. This is summarized in table 5.12 .

$$
\epsilon_{t}=\frac{N_{M C}}{20000}
$$




\begin{tabular}{|c|c|}
\hline Data events in region $\left(N_{D}\right)$ & 47 \\
\hline MC events in region $\left(N_{M C}\right)$ & 2272 \\
\hline Data events in background region $\left(N_{B}\right)$ & 5 \\
\hline
\end{tabular}

Table 5.12: Counts of ${ }^{212}$ Po candidates.

$$
\begin{gathered}
\sigma_{\epsilon_{t}}=\sqrt{\frac{\epsilon_{t}\left(1-\epsilon_{t}\right)}{N_{M C}}} \\
N_{212 B i}=\frac{N_{D}-N_{B}}{\epsilon_{t}} \\
\sigma_{N_{212} B i}=\sqrt{\frac{N_{D}}{\epsilon_{t}^{2}}+\frac{N_{B}}{\epsilon_{t}^{2}}+\left(\frac{\left(N_{D}-N_{B}\right) \sigma_{\epsilon_{t}}}{\epsilon_{t}^{2}}\right)^{2}}
\end{gathered}
$$

Equations 5.10 to 5.13 were used to estimate the ${ }^{212} \mathrm{Bi}$ activity based on the measured number of ${ }^{212}$ Po candidates. The input variables are defined in table 5.12 $\epsilon_{t}$ represents the efficiency or probability that a ${ }^{212}$ Po decay in the LAr will produce a trigger in the detector in the region of parameter space shown in Figures 5.9 and 5.10 . $\sigma_{\epsilon_{t}}$ is the statistical (binomial) uncertainty on $\epsilon_{t} . N_{212} B i$ is the estimated number of decays in the LAr volume, and $\sigma_{N_{212} B i}$ is the statistical uncertainty on $N_{212} B i$, calculated through standard error propagation.

From equation 5.10 and 5.11, the "efficiency" of producing these events in the Monte Carlo is $0.1141 \pm 0.0022$ per ${ }^{212} \mathrm{Bi}$ decay (assuming binomial errors).

From equation 5.12 and 5.13 , this results in $370 \pm 64{ }^{212} \mathrm{Bi}$ decays, assuming Poisson errors for the observed number of data events. With the $64.1 \%$ branching ration of ${ }^{212} \mathrm{Po}$, this corresponds to $237 \pm 41^{212} \mathrm{Po}$ decays.

The simple systematics study done in section 5.3.3 gave an upper systematic bound of 484 events and a lower bound of 343 events. Factoring this into the final result gives $370_{-70}^{+130}{ }^{212} \mathbf{B i}$ decays or $240_{-40}^{+80}{ }^{212}$ Po decays. 


\subsection{3 $\quad{ }^{212}$ Po Systematics}

The dominant source of uncertainty is expected to arise from setting the location of the line separating the ${ }^{214} \mathrm{Po}$ and ${ }^{212}$ Po populations in Figure 5.10 to match the corresponding line in Figure 5.9. This process was done by visual inspection of Figure 5.10. The systematic shifts shown in Figures 5.12 to 5.15 are extreme values beyond which the line clearly no longer corresponds to Figure 5.9 . The systematic uncertainty was assigned from the upper and lower extreme values of estimated ${ }^{212}$ Po decays obtained from the systematic shifts.

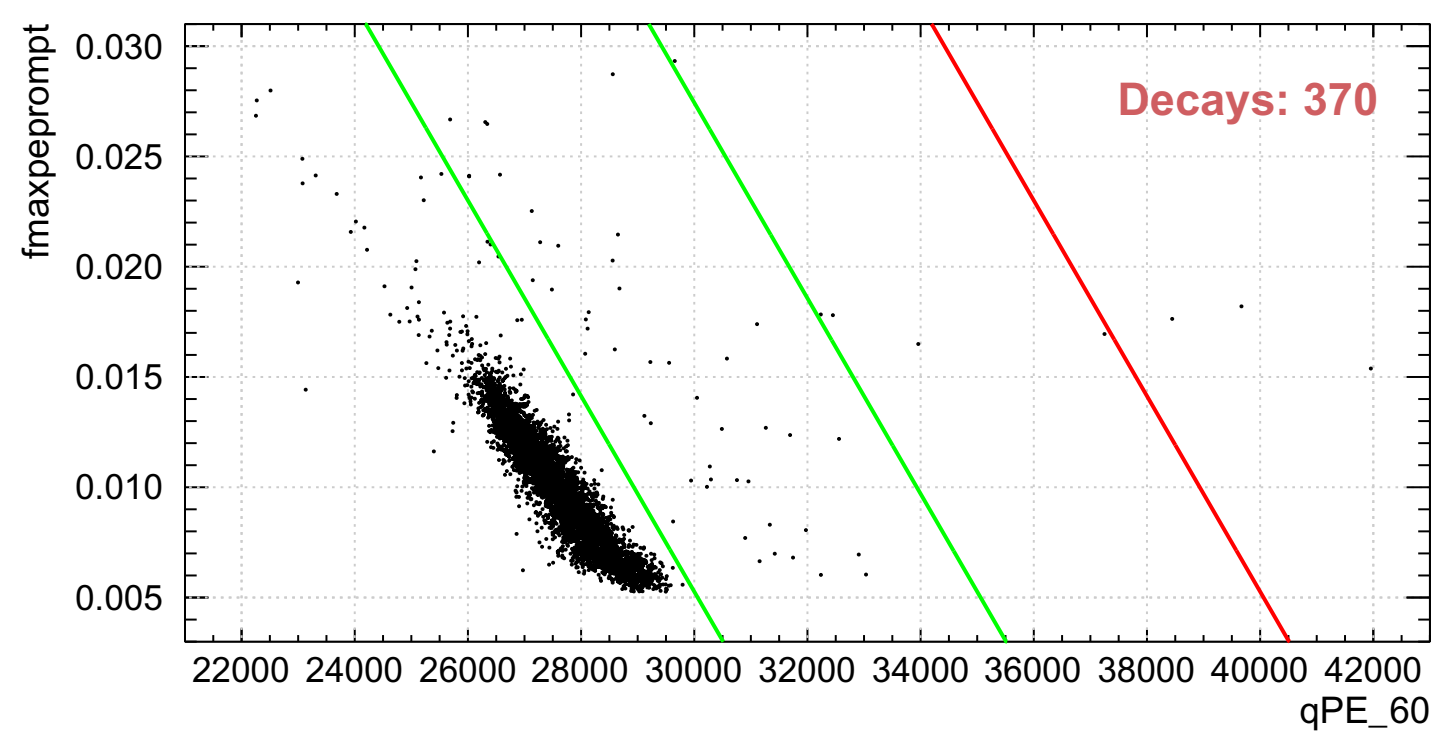

Figure 5.11: Cut used in computing central value of ${ }^{212} \mathrm{Bi}$ decay count estimate. 


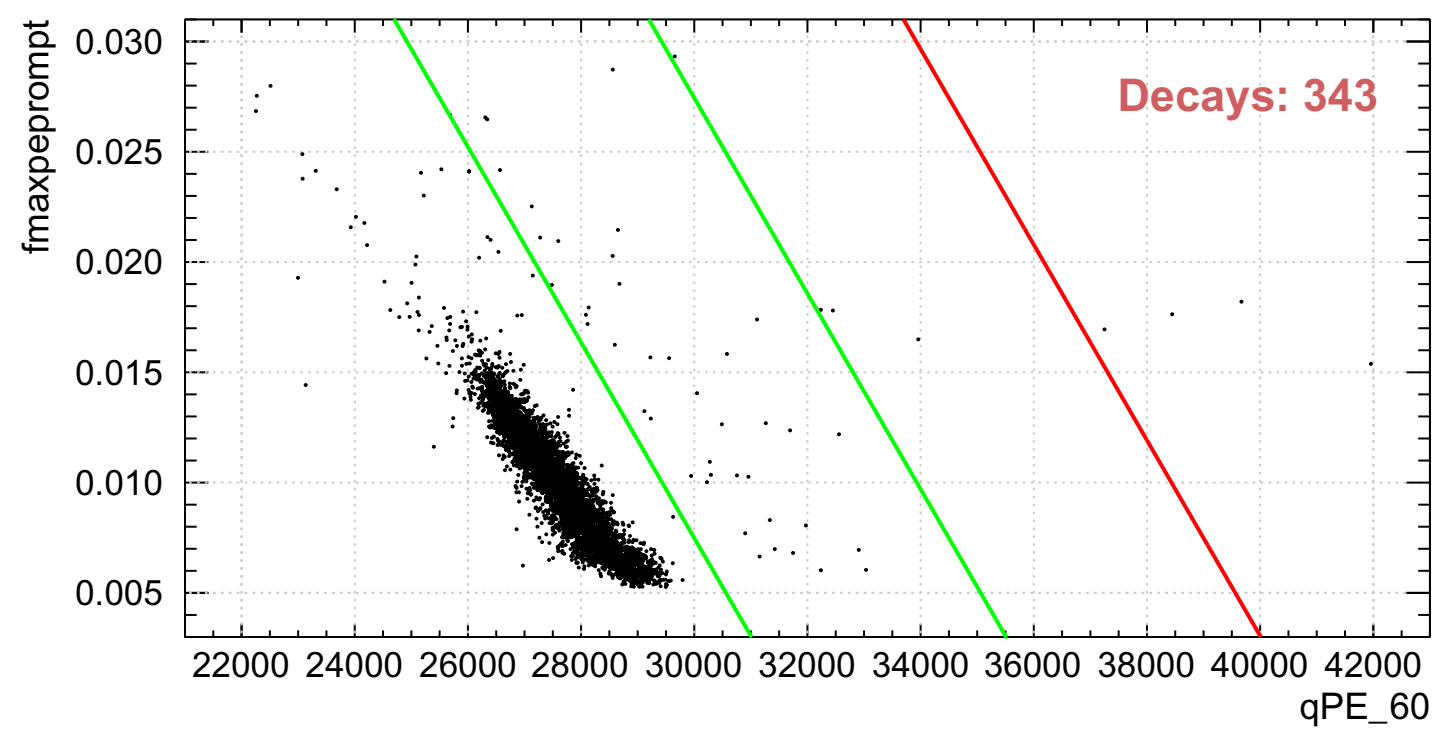

Figure 5.12: Shifted up 500 qPE_60.

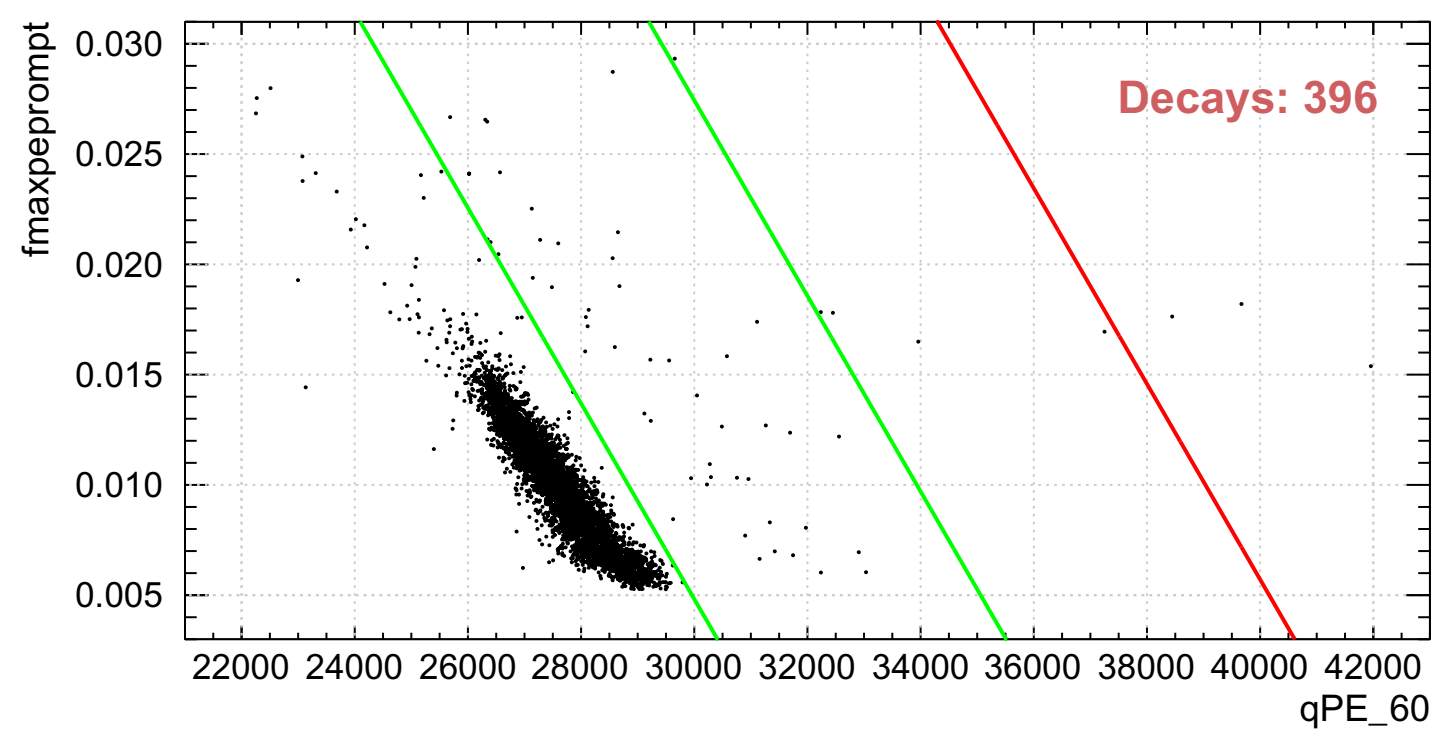

Figure 5.13: Shifted down 200 qPE_60. 


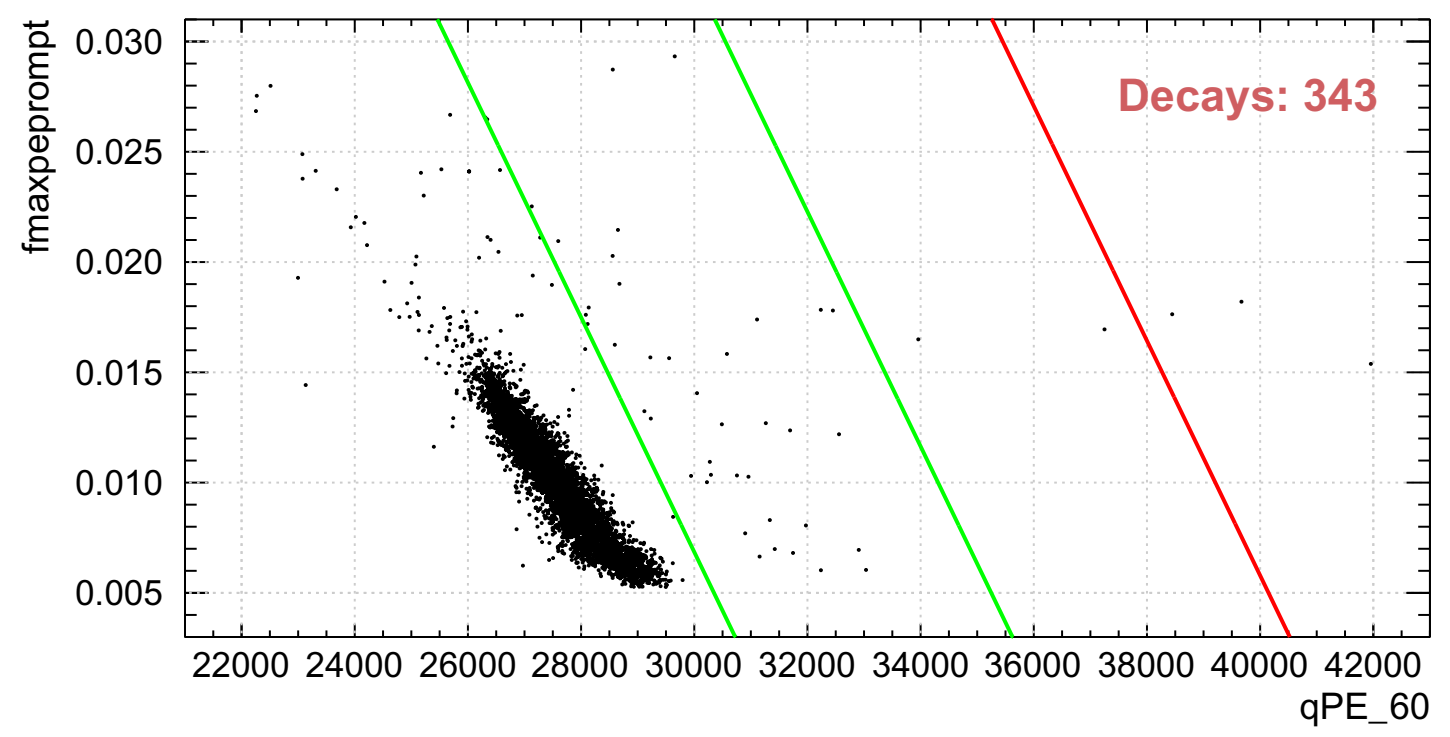

Figure 5.14: Slope increased.

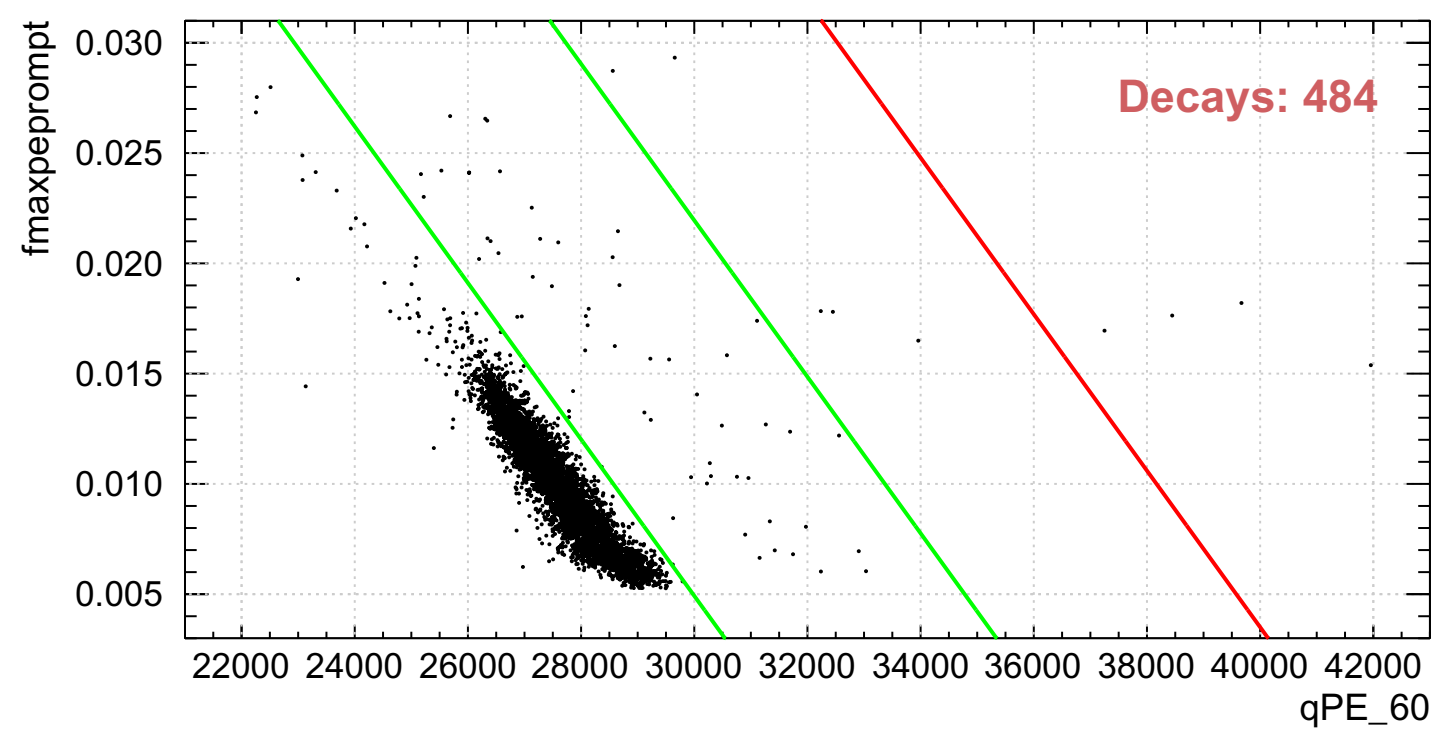

Figure 5.15: Slope decreased. 


\subsection{Time Coincidence Tagging of ${ }^{222} \mathrm{Rn}-{ }^{218} \mathrm{Po},{ }^{220} \mathrm{Rn}$ - ${ }^{216} \mathrm{Po}$, and ${ }^{214} \mathrm{Bi}-{ }^{214} \mathrm{Po}$}

\subsubsection{Introduction}

The relatively short half-lives of ${ }^{214} \mathrm{Po},{ }^{216} \mathrm{Po}$, and ${ }^{218} \mathrm{Po}$ allow these $\alpha$ decays to be tagged with the decays of their parents. This is done by identifying an event in an energy and fprompt range where one of these decays is expected, and looking in a time window prior to the given event for the decay of the parent. This time window is referred to as the "signal window". In order to get an estimate of the rate of uncorrelated events in the signal window, a search is done for events that occur following the ${ }^{214} \mathrm{Po}(1.5 \mathrm{~ms}$ time window $)$ or ${ }^{216} \mathrm{Po}(0.5 \mathrm{~s}$ time window $)$ candidate using a window of the same size as the signal window. This is referred to as the "random window" (see Figure 5.16). By applying appropriate cuts on the length of this time window and the reconstructed spatial separation of the tagged pairs, one can obtain a relatively clean sample of these $\alpha$ populations.

Once a tagged pair has been identified, the event variables describing each of the two tagged events, and an additional variable containing the separation in time between the pairs, are saved in a single entry in a ROOT "ntuple", a compressed file type that records the values of a set of parameters (qPE, fprompt, etc) for each entry. These ntuples are used for the analysis detailed in this section. Note that all low fprompt events that occur within the tagging window are counted, making it possible for a single high fprompt event to be tagged with multiple low fprompt events.

Note that all fits performed in this section are binned log-likelihood fits. 


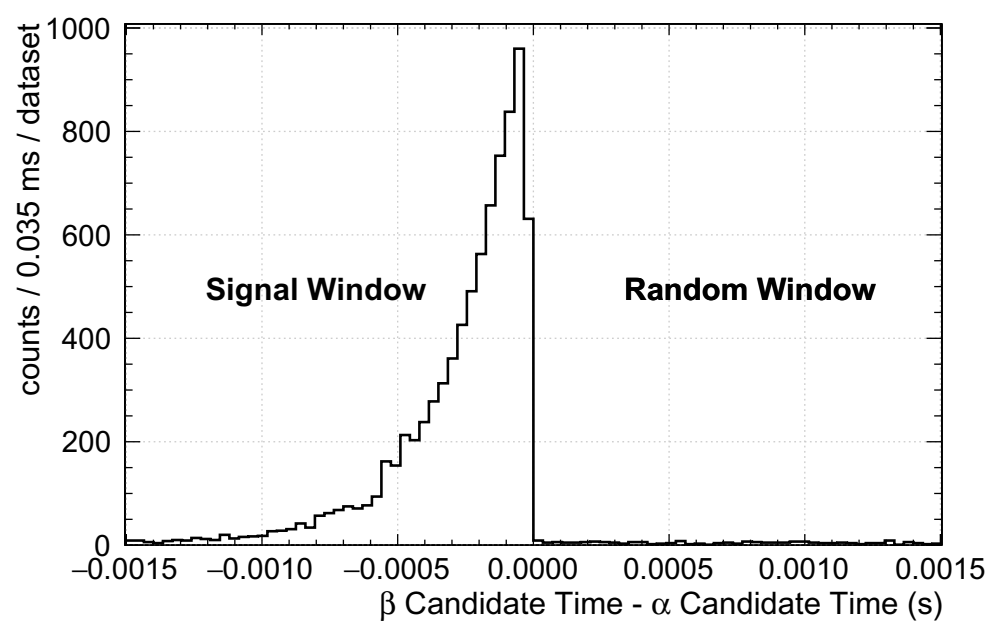

Figure 5.16: Sample spectrum of the coincidence tag time separation for ${ }^{214} \mathrm{Bi}(\beta$ decay) tagged with ${ }^{214} \mathrm{Po}$ ( $\alpha$ decay). The time difference is measured relative to the $\alpha$ candidate $\left({ }^{214} \mathrm{Po}\right.$ candidates). Therefore, since ${ }^{214} \mathrm{Bi}$ is the parent of ${ }^{214} \mathrm{Po}$, the ${ }^{214} \mathrm{Bi}$ decay signal window is on the negative axis.

\subsubsection{Tagging ${ }^{214} \mathrm{Bi}$ and ${ }^{214} \mathrm{Po}$}

${ }^{214}$ Po has a relatively short half-life of $164.3 \mu \mathrm{s}$. This makes it a good target for time coincidence tagging. This tagging is made complicated, however, by the fact that ${ }^{214} \mathrm{Bi}$ decays through a $\beta$ emission. $\beta$ particles are emitted with a very broad energy spectrum, which overlaps with the many other $\beta$ spectra in the detector. To mitigate this issue, the algorithm started with the $\alpha$ particle, which is part of a much cleaner distribution, and then searched for the $\beta$ that should have occurred prior to it. In addition, a lower limit is used on the $\beta$ energy. This causes the tagging algorithm to miss some ${ }^{214}$ Po decays, but significantly reduces the amount of random time tags that would arise from tagging with the ${ }^{39} \mathrm{Ar}$, which has a rate of $3120 \pm 190 \mathrm{~Hz}$ in DEAP-3600 [1]. This $\beta$ energy threshold is set at 4000 qPE (approximately $550 \mathrm{keV}$ ). For reference, $1 \mathrm{keV}$ is roughly $7.3 \mathrm{qPE}$ for electron and $\gamma$ events.

The main purpose of this analysis is to identify the $\alpha$ populations in qPE space. For ${ }^{214} \mathrm{Po}$, we would expect that a distribution may exist in the following regions: 
- The liquid argon bulk (high qPE). This is referred to as the "full energy" peak.

- The liquid argon and TPB interface (spread in qPE from the full energy liquid argon peak down to the detection threshold)

- The gaseous argon

- The neck

The cuts used to tag $\alpha$ candidates were:

- $0.6<$ fprompt $<\mathbf{0 . 8}$ : Selects high fprompt (nuclear recoil) events

- Standard low level cuts: Removes hardware related events (see Appendix A)

- Standard pileup cuts: Removes pileup (see Appendix A

- fmaxpe $<\mathbf{0 . 4}$ : Removes Cerenkov

The cuts used to tag $\beta$ candidates were:

- $0.2<$ fprompt $<$ 0.4: Selects low fprompt (electron recoil) events

- $\mathbf{q P E}>$ 4000: Selects events above ${ }^{39} \mathrm{Ar}$ energies

- Standard low level cuts: Removes hardware related events (see Appendix A)

- Standard pileup cuts: Removes pileup (see Appendix A)

- fmaxpe $<\mathbf{0 . 4}$ : Removes Cerenkov

The numbers of events in various qPE ranges are shown in table 5.13. Plots of the qPE spectrum for these tags overlaid with all of the high fprompt data are shown in Figure 5.17. The corresponding plots zoomed-in to the ROI are shown in Figure 5.18. The peak starting around $35000 \mathrm{qPE}$ is the ${ }^{214} \mathrm{Po}$ full energy peak. The qPE spectrum can be seen with finer binning in Figure 5.19, with the full-energy $\alpha$ peaks more visible. 
All High fprompt Data After Low Level and Pileup Cuts

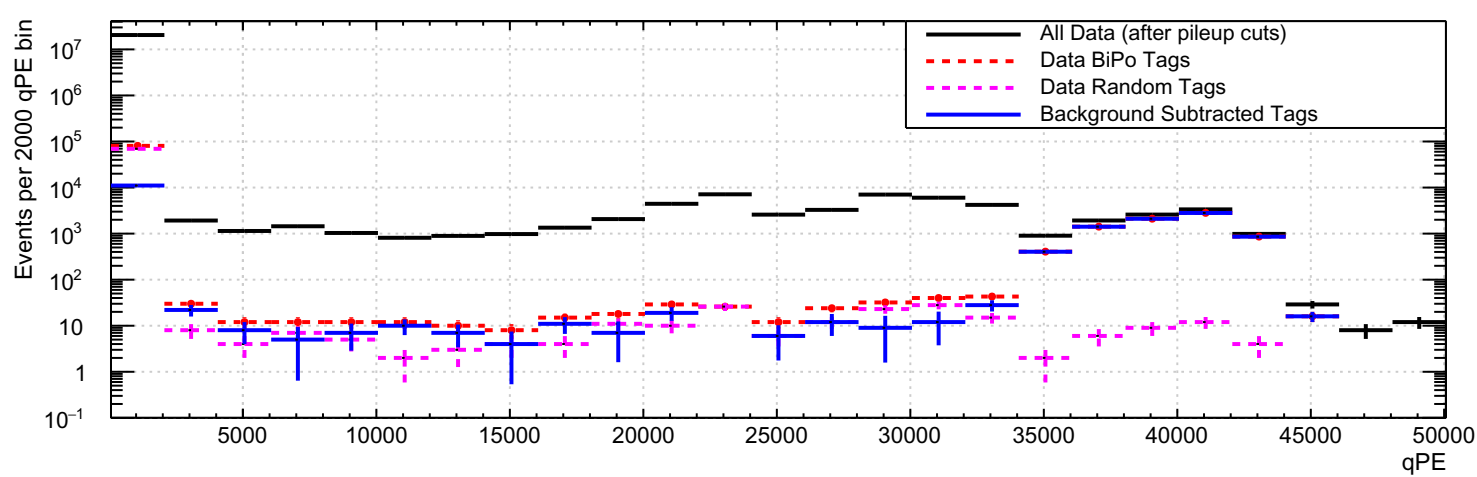

After fmaxpe $<0.4$

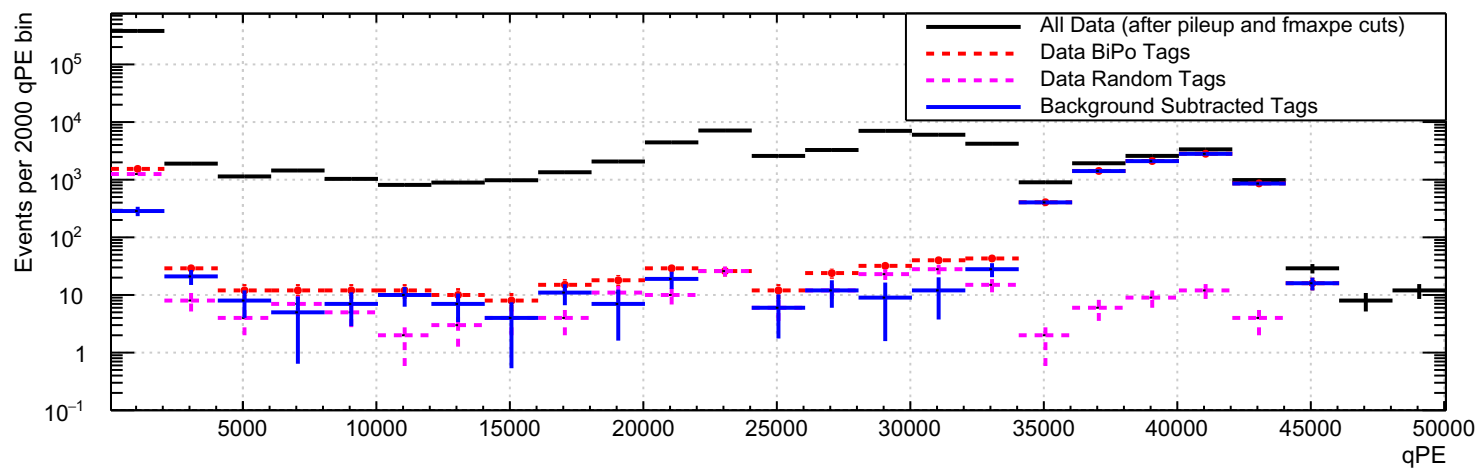

After fmaxpe $<0.4$ and tag separation $<200 \mathrm{~mm}$

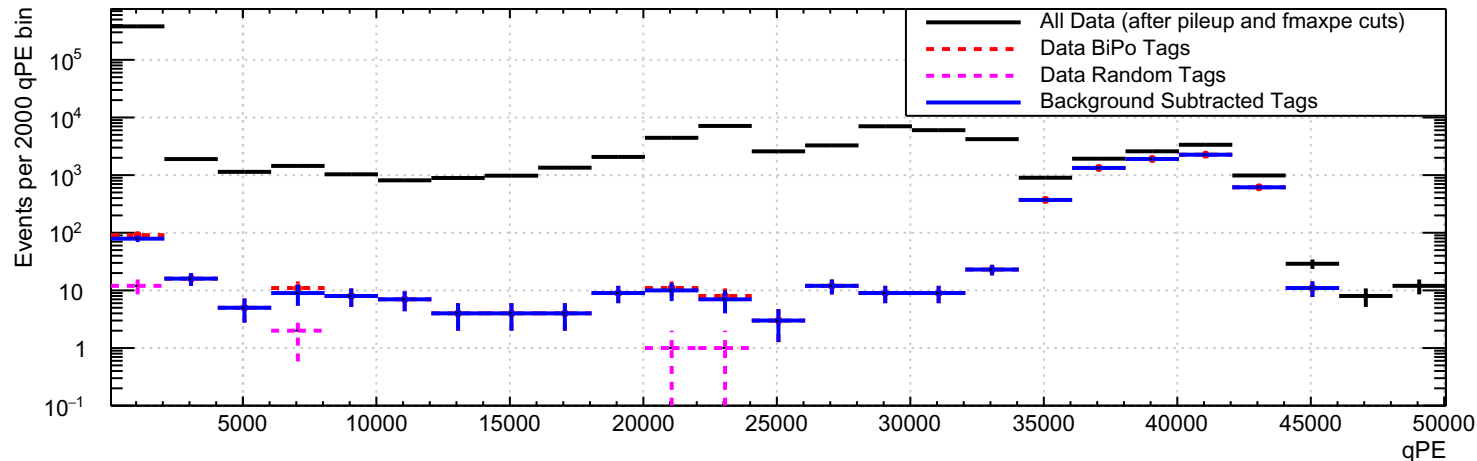

Figure 5.17: The qPE spectrum for individual high fprompt events and high fprompt events that occurred within $0.0015 \mathrm{~s}$ of a low fprompt event with qPE $>4000$. The top plot shows all events after standard low level and pileup cuts, the centre plot adds an fmaxpe cut, and the bottom plot adds a cut on the spatial tag separation. 
All High fprompt Data After Low Level and Pileup Cuts

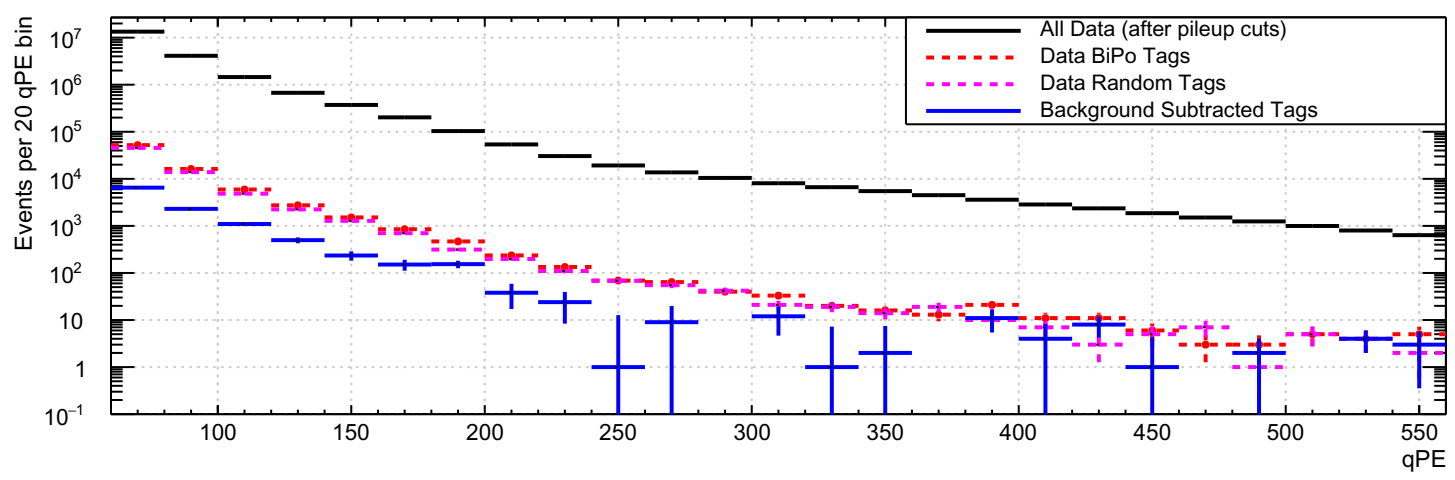

After fmaxpe $<0.4$

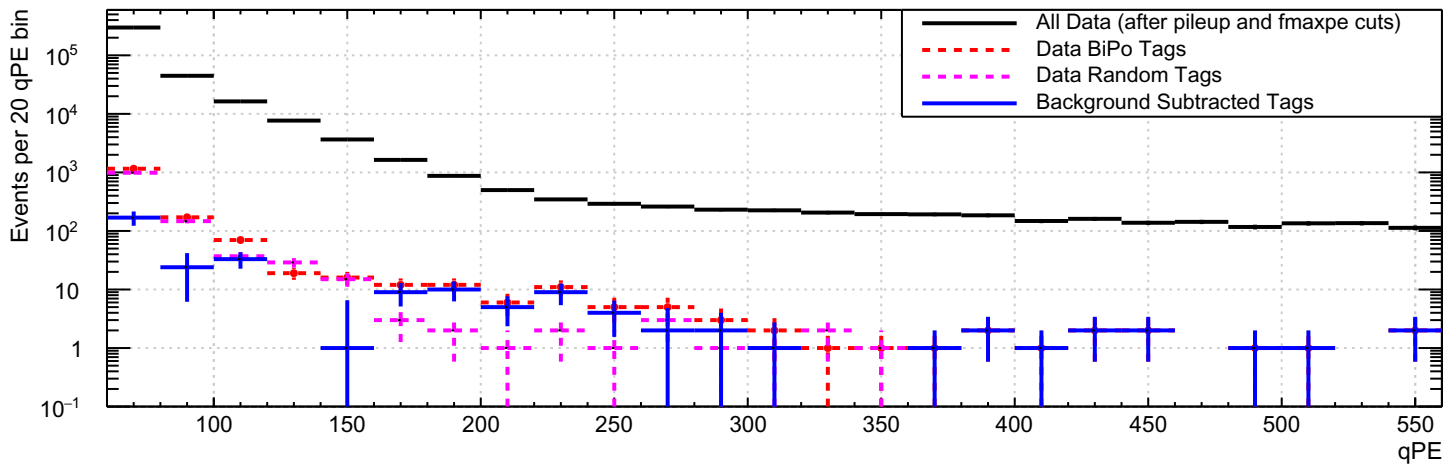

After fmaxpe $<0.4$ and tag separation $<200 \mathrm{~mm}$

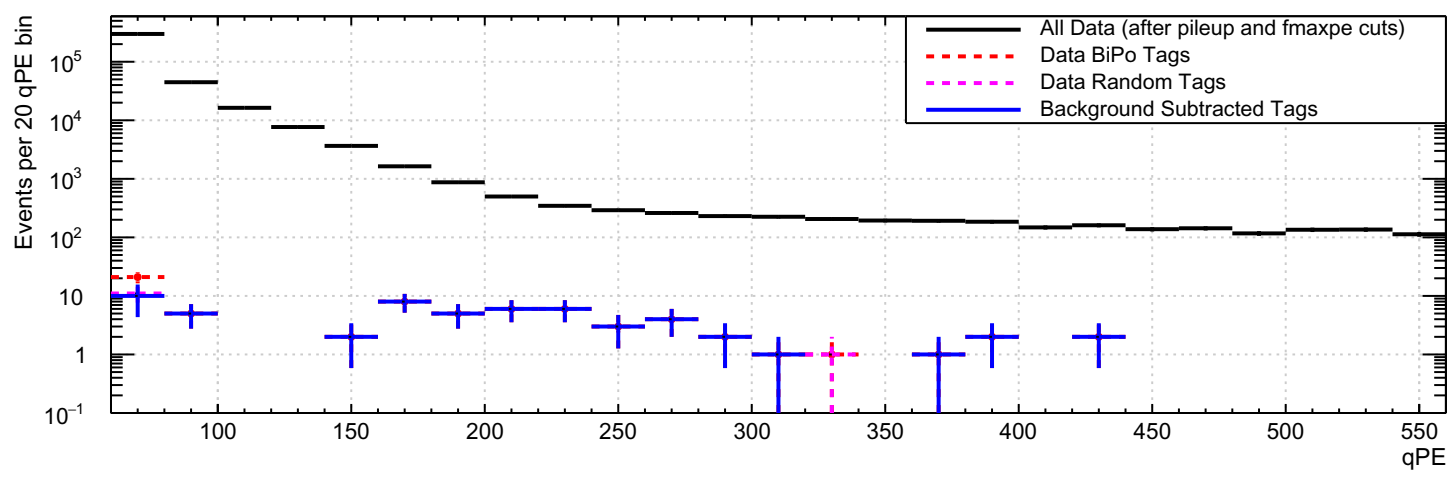

Figure 5.18: Same as Figure 5.17, but zoomed-in to WIMP ROI-scale energies. 


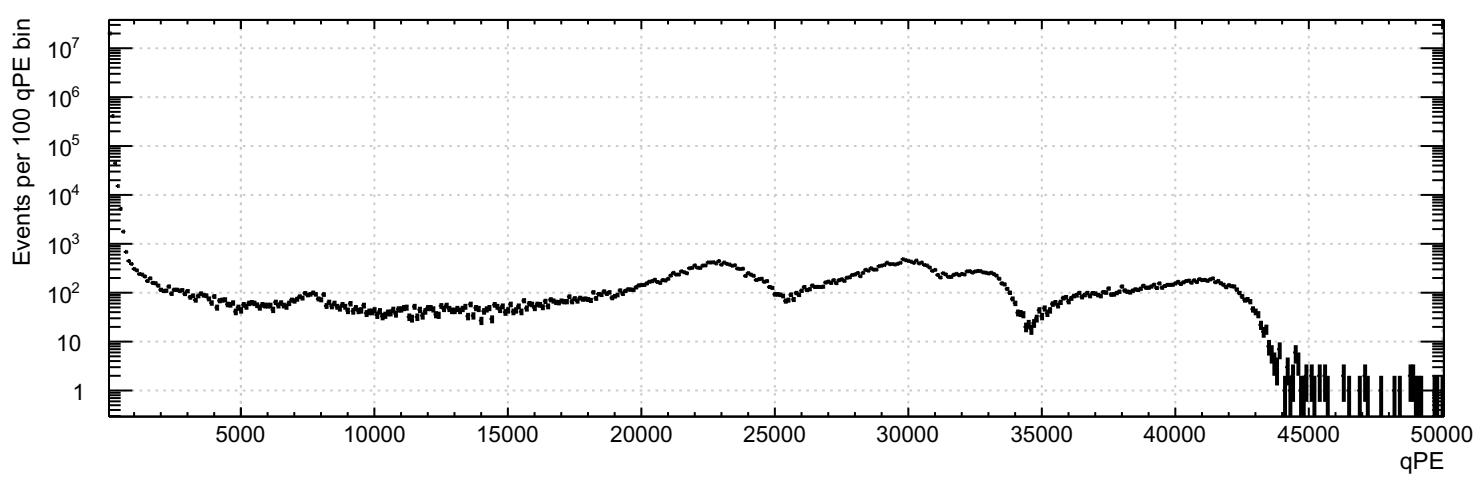

Figure 5.19: All data events after standard low level and pileup cuts, shown with 100 qPE binning.

\begin{tabular}{|c|c|c|c|c|}
\hline qPE Range & \multicolumn{2}{|c|}{ Signal Counts } & \multicolumn{2}{c|}{ Random Counts } \\
& sep $<200$ & no sep cut & w sep $<200$ & no sep cut \\
\hline${ }^{214}$ Po peak & 6495 & 7627 & 0 & 32 \\
$25,400-{ }^{214}$ Po peak & 55 & 145 & 0 & 80 \\
$14,000-25,400$ & 38 & 106 & 2 & 60 \\
$6,000-14,000$ & 31 & 47 & 2 & 17 \\
$500-6,000$ & 42 & 80 & 0 & 33 \\
$240-500$ & 16 & 26 & 1 & 9 \\
$100-240$ & 27 & 139 & 0 & 87 \\
$0-100$ & 206 & 16969 & 208 & 17365 \\
\hline
\end{tabular}

Table 5.13: Raw numbers of tagged high fprompt events in various qPE ranges. The cuts used here are the standard low level and pileup cuts and fmaxpe < 0.4. The data in this table is shown graphically in Figures 5.17 and 5.18. These numbers can be used with the tagging efficiency to calculate the amount of ${ }^{214} \mathrm{Po}$ decay events present in each qPE region. The ${ }^{214} \mathrm{Po}$ starts around 35,000 qPE and ends around 44,000 qPE. 


\section{${ }^{214}$ Po Tags in the Liquid Argon Bulk}

The liquid argon ${ }^{214} \mathrm{Po}$ peak is clearly visible between 35,000 and 44,000 qPE in figure 5.20. The time between tags in this peak was plotted, and the lifetime was fit (see figure 5.21). The fitted lifetime of $239.1 \pm 3.1 \mu$ s was consistent with the expected lifetime of $237.0 \pm 2.9 \mu$ s for ${ }^{214} \mathrm{Po}[57$. The uncertainties on the results of the decay time fits only include the statistical uncertainties as calculated by the fitting algorithm (Minuit) using a Poisson likelihood function.

There is no other known background that will contribute to the signal window but not the random window. Other time correlated events, such as neutron capture $\gamma$ events and neutron scatter in the LAr will produce a scatter (high fprompt) followed by a $\gamma$ (low fprompt), which is the opposite to from what occurs in Bi $/{ }^{214}$ Po events. Neutron scatters also produce events with much lower energies than $\alpha$ decays. It is, therefore, assumed that there is no significant contamination of the signal window, at least at the full $\alpha$ decay energies. This assumption seems to be valid given the fact that the fitted lifetime of ${ }^{214}$ Po matches quite well with the expected value.

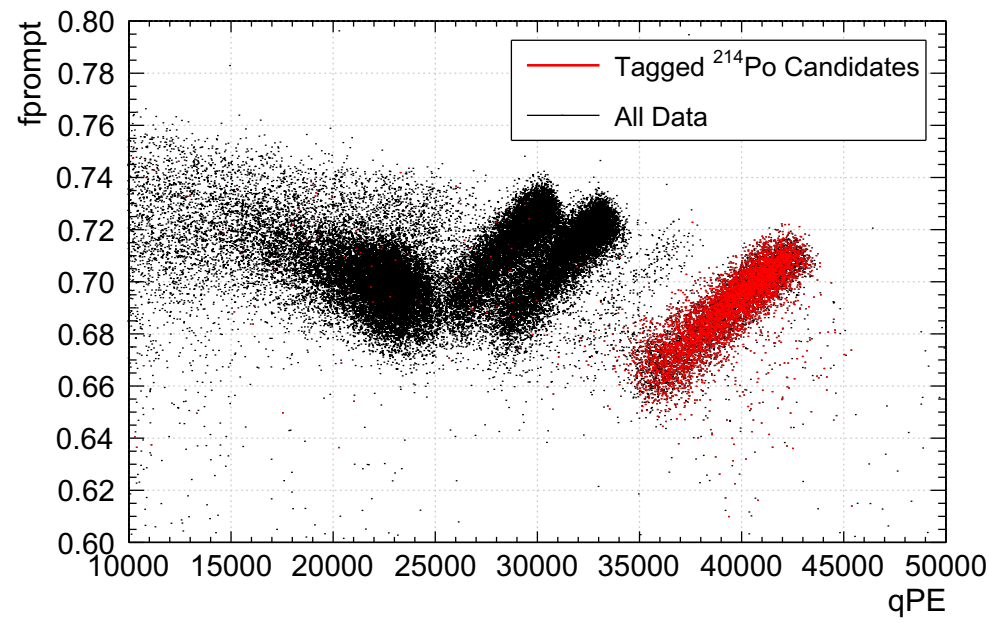

Figure 5.20: fprompt vs qPE distribution of tagged ${ }^{214} \mathrm{Po}$ events (red) overlaid with the rest of the data (black). The live-time here (and elsewhere in this analysis) is 247.17 -days. 


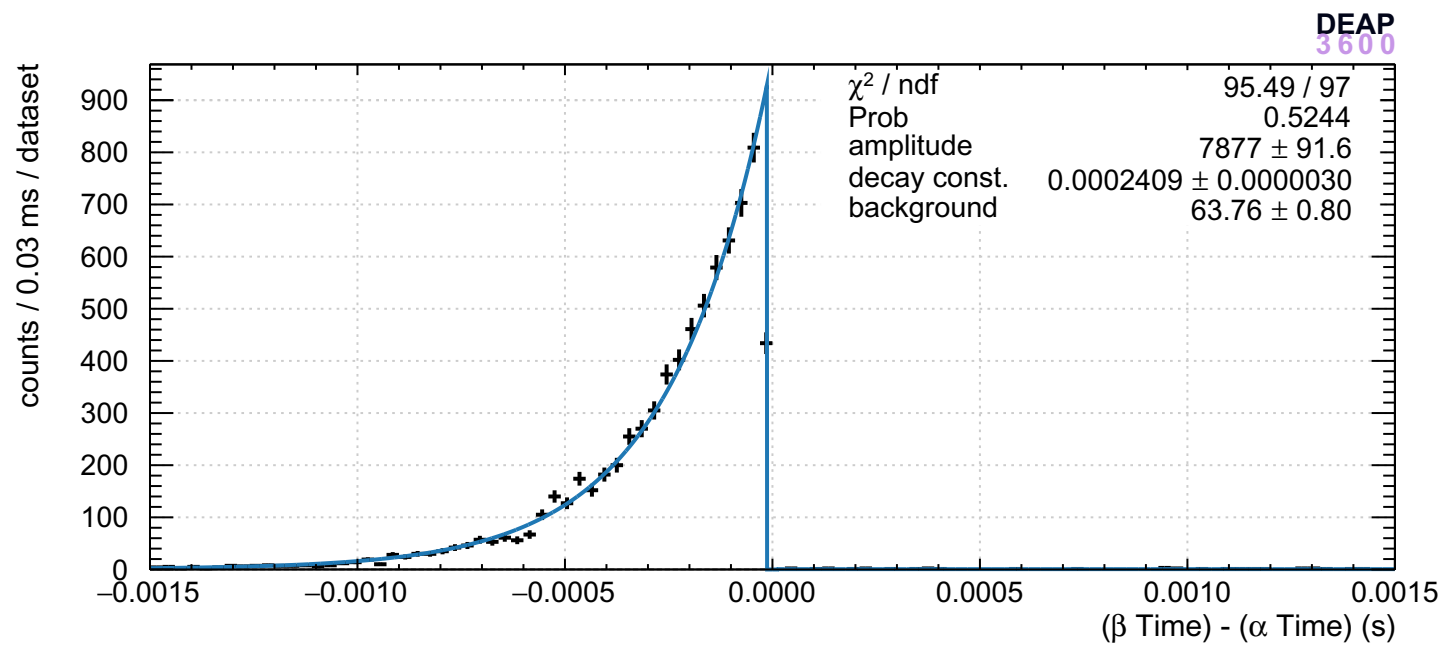

Figure 5.21: Distribution of the time between tagged pairs in which the $\alpha$ candidate is located in the ${ }^{214}$ Po peak in fprompt vs qPE space. Standard low level and pileup cuts were applied to both the $\alpha$ and $\beta$ candidates. The lifetime was fitted to be $239.1 \pm 3.1 \mu \mathrm{s}$, which is consistent with the accepted value of $237.0 \pm$ $2.9 \mu$ s for the ${ }^{214}$ Po lifetime. Note that all low fprompt events that occur within the tagging window are included in this analysis.

\section{Tagging Efficiency of Full Energy Bi $/{ }^{214}$ Po Events in the LAr}

Tagging efficiency is defined here as the fraction of the time that a ${ }^{214} \mathrm{Po}$ decay is tagged with the decay of its parent, ${ }^{214} \mathrm{Bi}$. It can be estimated using a pure sample of ${ }^{214}$ Po decays by dividing the number of those events that are tagged with ${ }^{214} \mathrm{Bi}$ decays by the total number of ${ }^{214}$ Po events in the pure sample. For the purpose of calculating the tagging efficiency, $\mathrm{MC}$ events were generated with ${ }^{214} \mathrm{Bi}$ isotopes as the primary particle. ${ }^{214} \mathrm{Bi}$ has a half-life of 20 minutes, so to speed up the MC and reduce the $\mathrm{MC}$ event length, the ${ }^{214} \mathrm{Bi}$ half-life was modified to be $1 \mathrm{~ns}$. The tagging algorithm was run on the MC events. MC events with $0.6<$ fprompt $<0.8$ and $30,000<$ qPE $<50,000$ and fmaxpe $<0.4$ were selected, and the standard pileup cuts were applied. The efficiency was calculated by dividing the number of tagged events that passed these cuts by the total number of events that passed these cuts. This is shown as a function of qPE in Figure 5.22 . The overall tagging efficiency for $\mathrm{Bi} /{ }^{214} \mathrm{Po}$ decays in 
the liquid argon was $0.9281 \pm 0.0023$.

The large spread in energy in Figure 5.22 is due to clipping of the prompt charge peak in the digitizers, resulting in a reduction of observed charge for events where a large amount of energy is deposited in a few PMTs. These are primarily events that occur close to the surface. Therefore, events that occur close to the surface tend to have a lower measured energy. In these events, there is also a higher probability that the $\beta$ will be lost to the surface of the detector and either not trigger, or have an energy below the threshold for tagging. This results in a lower tagging efficiency.

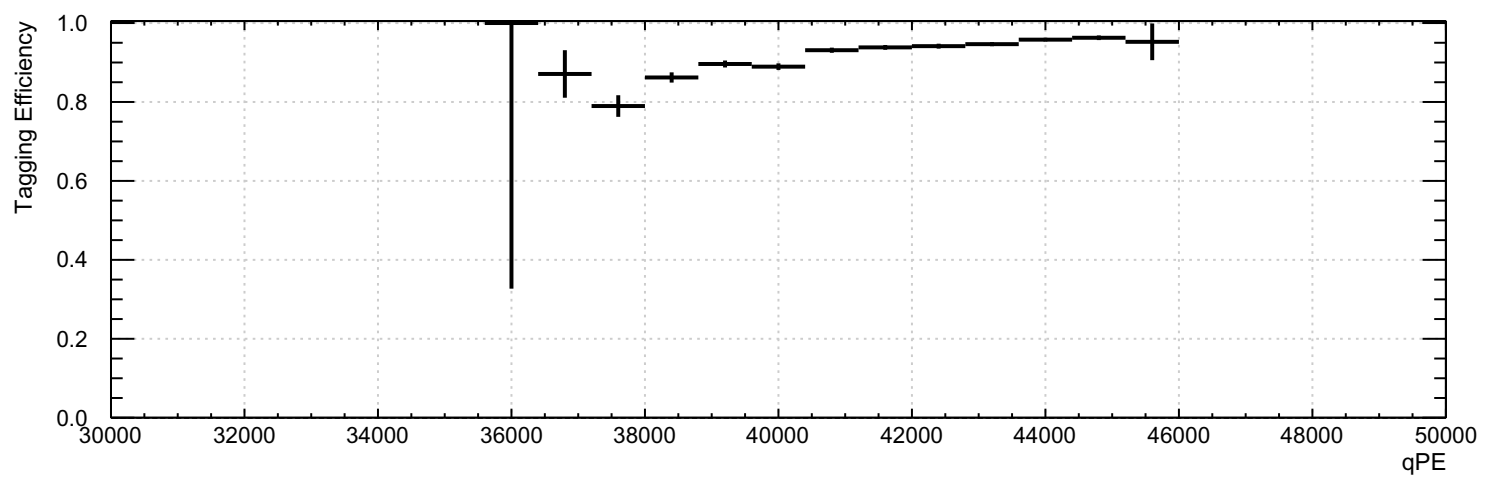

Figure 5.22: The tagging efficiency of $\mathrm{MC}{ }^{214} \mathrm{Po}$ events in the liquid argon as a function of the qPE of the ${ }^{214} \mathrm{Po}$ event.

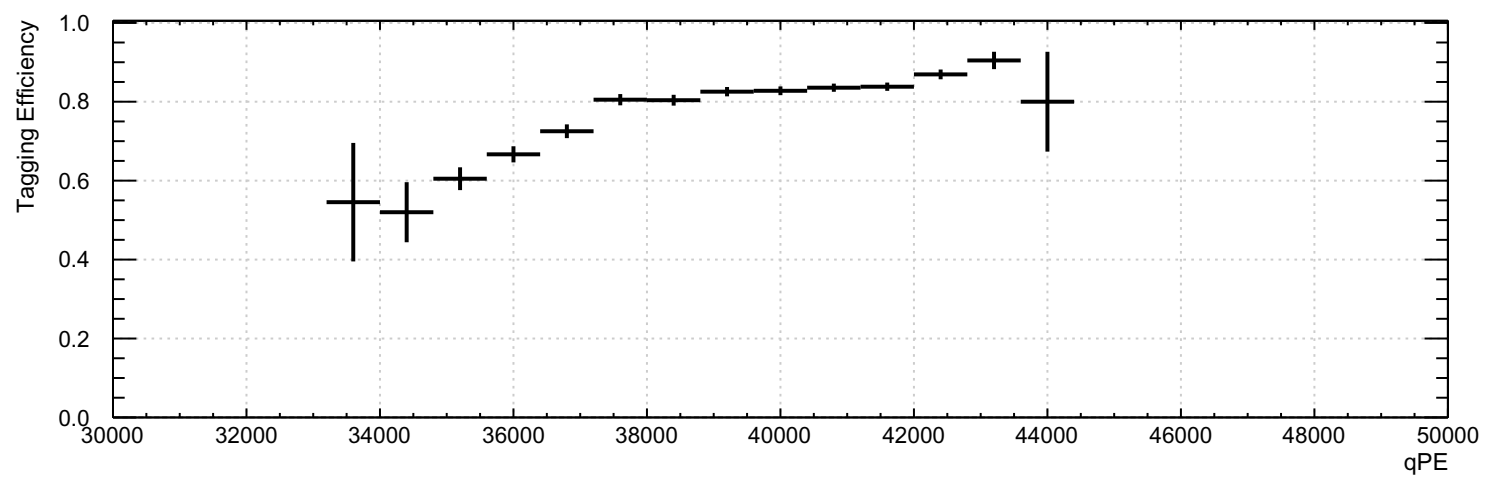

Figure 5.23: The tagging efficiency of Data ${ }^{214} \mathrm{Po}$ events in the liquid argon as a function of the qPE of the ${ }^{214} \mathrm{Po}$ event. The spectrum curves down at lower energy, likely due primarily to the presence of surface decays. 
The data tagging efficiency was calculated in the same way, but with the addition of the standard low-level cuts and the cuts (see Section A) to select ${ }^{214}$ Po events in the data. The tagging efficiency in data was $0.8045 \pm 0.0042$. It is shown as a function of qPE in Figure 5.23. It was calculated by making a histogram of the qPE of the ${ }^{214} \mathrm{Po}$ tags in the ${ }^{214} \mathrm{Po}$ box, and dividing it by a histogram of the qPE of all the events in the ${ }^{214}$ Po box.

The data and MC tagging efficiencies are not compatible. This is likely due to the presence of surface decays within the ${ }^{214}$ Po selection box. As will be discussed in the next section, these events have a lower tagging efficiency due to the loss of the $\beta$ to the inner surface of the detector. It is also likely that pileup with ${ }^{39} \mathrm{Ar}$, which has a rate of $3120 \pm 190 \mathrm{~Hz}$, may also reduce the number of low fprompt events that pass pileup cuts.

\section{${ }^{214}$ Po Tags on the TPB Surface}

The surface decay candidates are more difficult to find. Due to partial deposition of their energy in the liquid argon, the reconstructed energies of these events (which assumes a full deposition of energy in the LAr) extend from the liquid argon peak down to the detection threshold (the case when the $\alpha$ decays straight into the TPB and acrylic). The loss of energy to the TPB and/or the acrylic causes their (degraded) reconstructed energies to overlap with the reconstructed energies of other $\alpha$ distributions (especially with ${ }^{210} \mathrm{Po}$ ). In addition, there is a very high rate of events at low reconstructed energy that are correlated with high energy events (Figure 5.24). These low qPE events are suspected to be due to Cherenkov and re-triggers on the residual light in the detector following high energy low fprompt events. These events are also tagged by the time coincidence algorithm, as they occur shortly after a high energy $\beta$ event. It has proven very challenging to distinguish between these events and ${ }^{214}$ Po tags. To exclude these anomalous events, a cut is typically made to only 


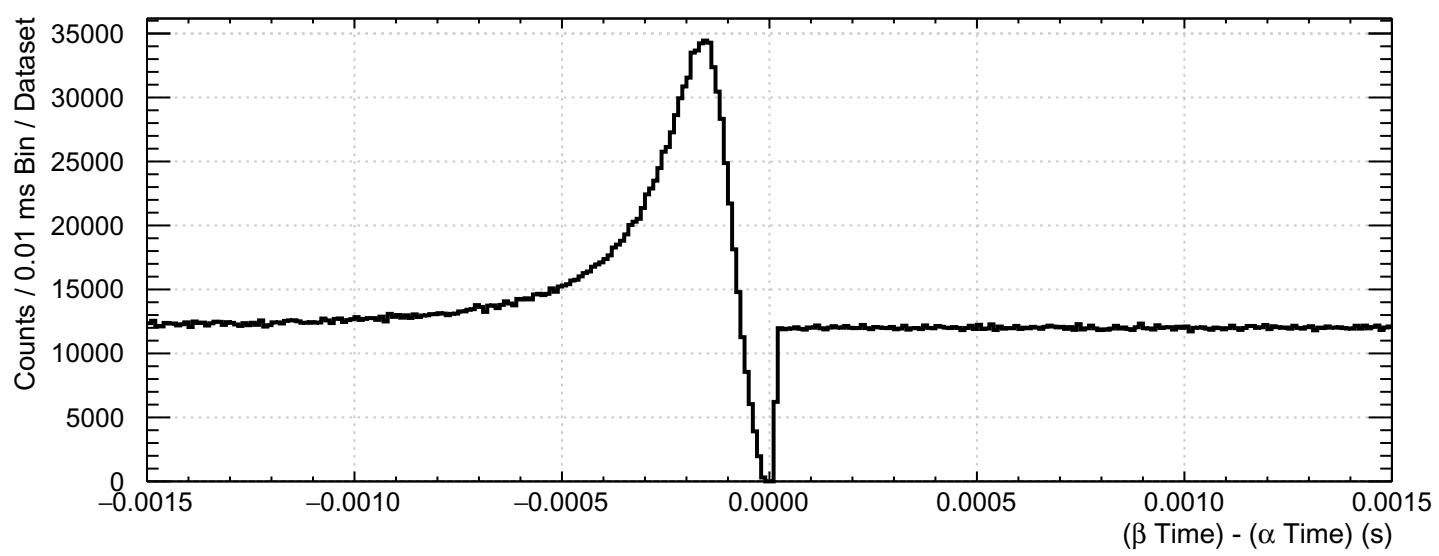

Figure 5.24: The tag time separation spectrum for events where the high fprompt tag has $\mathrm{qPE}<100$. The deficit here is due to the tail of the initial high energy low fprompt event leaking into the window of events that occur shortly after. This tail reduced the fprompt of the low energy high fprompt events that would normally occur in place of the deficit, drawing them below the fprompt $>0.6$ threshold for the high fprompt band in the tagging algorithm. The only cut used here was to restrict the high fprompt tag to have qPE $<100$. No pileup, low-level, or fmaxpe cuts were applied here.

include events with qPE $>100$.

${ }^{39}$ Ar decays can also produce false tags (referred to as "random" tags). These are thought to dominate the flat background component in the time spectrum fits.

The number of random coincidences is relatively high, however (see Figure 5.25). The spatial separation of the tagged pairs provided an additional discriminant on whether the two events originated from the same source particle. The spatial separations for tagged pairs of events in the signal window is plotted as the red histogram in Figure 5.27. The spatial separation of tagged pairs in the random window for events with $100<\mathrm{qPE}<{ }^{214} \mathrm{Po}$ box are plotted in the black histogram. The green line shows the location of the $200 \mathrm{~mm}$ cut that was used to reduce the number of random coincidence events, resulting in the time separation spectrum shown in Figure 5.26. This cut has a signal acceptance of $0.852 \pm 0.004$ and a random coincidence acceptance of $0.012 \pm 0.001$ 


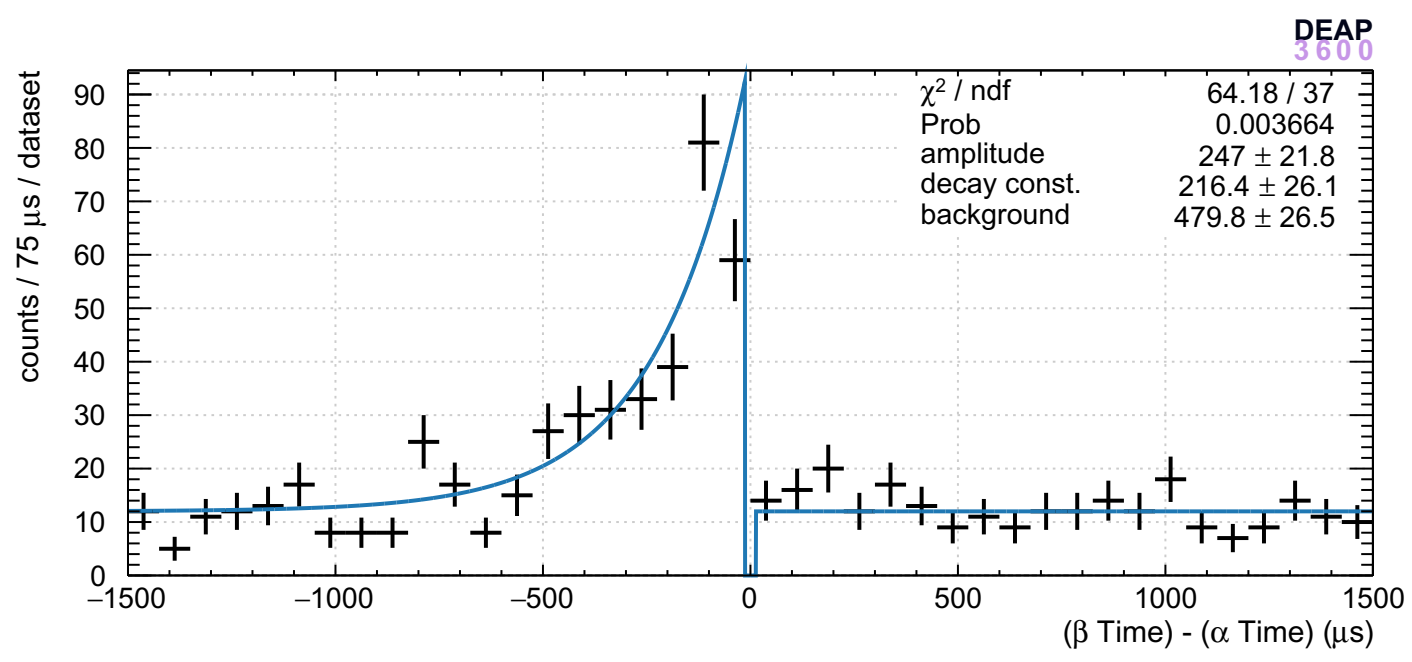

Figure 5.25: The tag time separation spectrum for events where the high fprompt tag has $100<\mathrm{qPE}<{ }^{214} \mathrm{Po}$ Peak.

Placing a cut at $200 \mathrm{~mm}$ separation results in the time separation spectrum shown in Figure 5.26. As seen in the figure, there are now very few events in the random window compared with the signal window. If these represent real ${ }^{214} \mathrm{Po}$ decays, then this would imply that the reconstructed energies of these events are being degraded somehow. The fitted lifetime was $230 \pm 20 \mu \mathrm{s}$, which is consistent with the $237.0 \pm$ $2.9 \mu$ s expected lifetime of ${ }^{214} \mathrm{Po}$.

Figure 5.28 shows the $2 \mathrm{D}$ projection of the reconstructed positions of ${ }^{214}$ Po surface tag candidates onto the surface of the AV. The main feature to note here is that there seems to be a concentration of events near the bottom of the detector. This is consistent with the idea (discussed later) that dust is present in the LAr, since one would expect that dust could settle on the bottom if its density is greater than that of liquid argon.

In order to get an estimate on the probability of a surface ${ }^{214}$ Po event leaking into the WIMP ROI, we need an estimate of the rate of these events. However, there is some inefficiency and uncertainty in the number of ${ }^{214} \mathrm{Po}$ tags. Unfortunately, the Monte Carlo does not model the roughness of the TPB surface. This makes a 


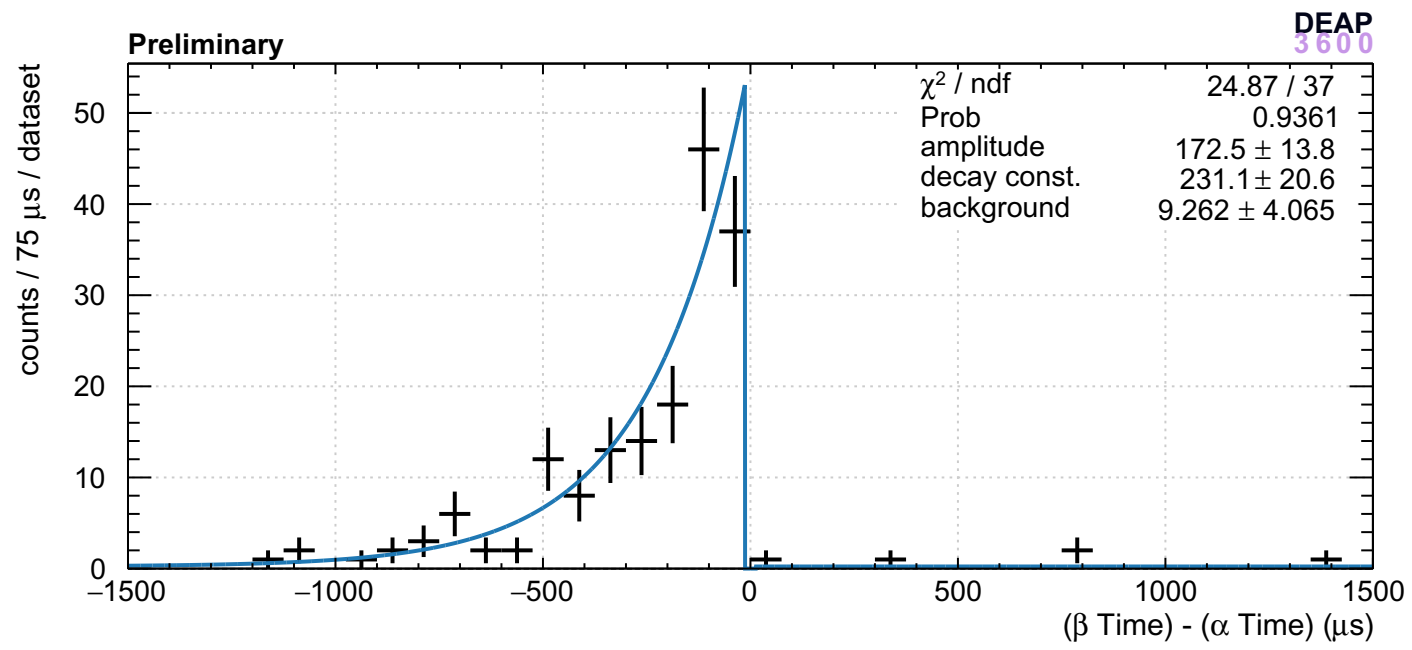

Figure 5.26: Distribution of time between $\mathrm{Bi}^{214} \mathrm{Po}$ tags with $100<\mathrm{qPE}<{ }^{214} \mathrm{Po}$ Peak, and the requirement that tagged events be within $200 \mathrm{~mm}$ of each other. Standard low-level and pileup cuts were applied, and a cut selecting events with fmaxpe $<0.2$. Note that about $15 \%$ of the events in this plot have fmaxpe $<$ 0.035, which is significantly below Monte Carlo predictions for surface events. The "amplitude" and "background" fit parameters are in units of counts/dataset, and the decay constant is in units of $\mu \mathrm{s}$.

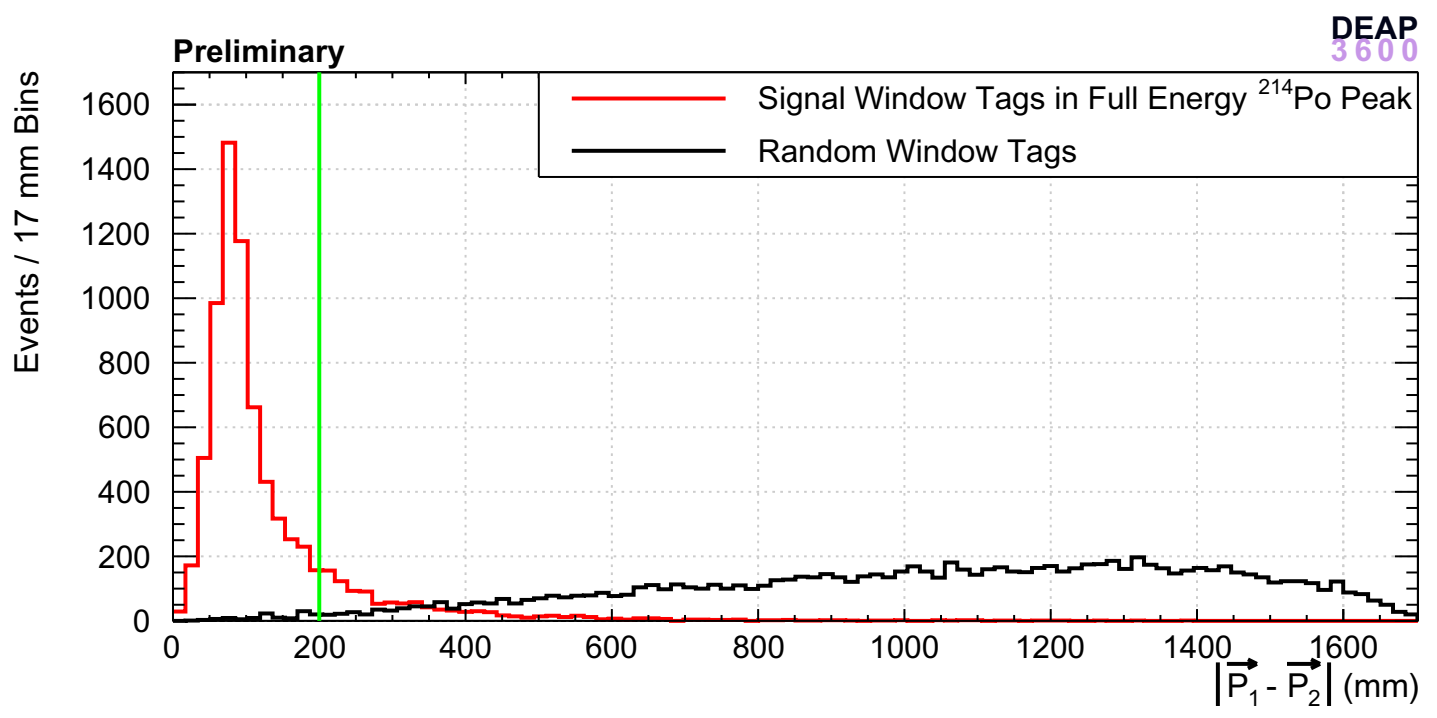

Figure 5.27: Distribution of spatial separation between time-coincidence tags with a coincidence window of $0.0015 \mathrm{~s}$. This is the nominal window length for tagging $\mathrm{Bi}^{214} \mathrm{Po}$ events. The red line is the distribution for signal window tags in the full energy ${ }^{214} \mathrm{Po} \alpha$ peak. The black line is the distribution for random window tags inside and outside the full energy ${ }^{214} \mathrm{Po} \alpha$ peak and with qPE $>100$. 


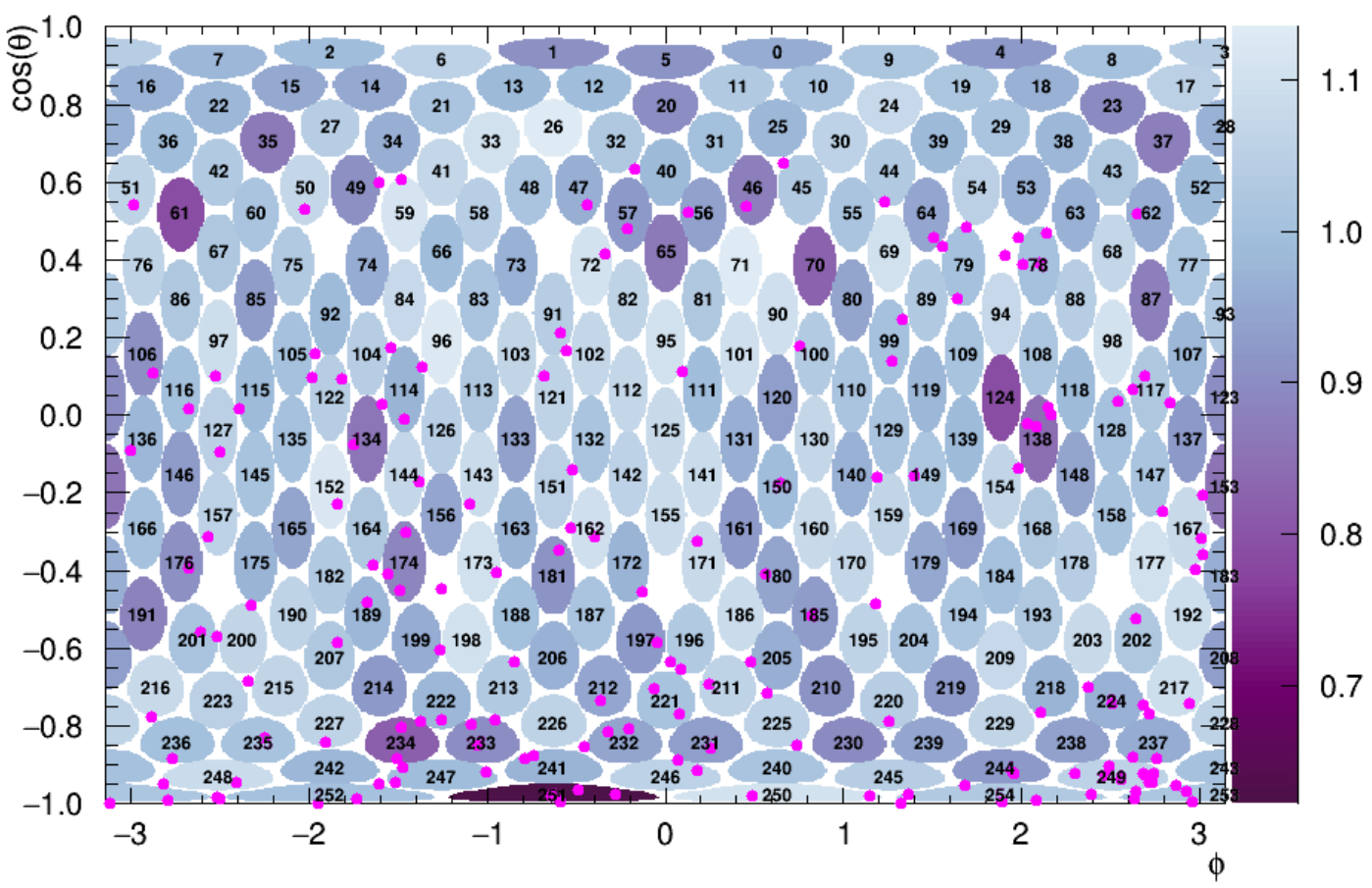

Figure 5.28: PMT map showing the efficiencies of the PMTs on a colour scale. Overlaid on this map are the $2 \mathrm{D}$ projections of the locations of the ${ }^{214}$ Po surface candidate events in the dataset. The cuts and energy range used here are the same as in Figure 5.26, but with only events in the signal window included. 
calculation of the absolute rate difficult. It is still possible to reconstruct the number of ${ }^{214} \mathrm{Po}$ events in a given energy range, however. This is done by first measuring tagging and cut efficiencies in the Monte Carlo for various qPE ranges (table 5.14). The tagged ${ }^{214} \mathrm{Po}$ candidates are counted for each energy range (table 5.15). The background is estimated using the random window, and then subtracted from the number of tags in the qPE range. This number is finally corrected using the tagging and cut efficiencies to yield an estimate of the number of ${ }^{214} \mathrm{Po}$ events in a given $\mathrm{PPE}$ range (table 5.16). This assumes that the tagging and cut efficiencies are constant across qPE ranges (see Figure 5.29.

The uncertainties in table 5.14 were calculated using the TEfficiency class in ROOT, which uses the Clopper-Pearson interval method. The uncertainties in table 5.16 were calculated using standard error propagation techniques, assuming a Poisson uncertainty on the number of counts in each window. This was compared with the more rigorous calculation in [58], which is implemented as the TRolke class in ROOT. However, this class gave invalid results for cases where the random window contained 0 events, so it was decided to not use this method in calculating the results in table 5.16. The results produced by TRolke were generally close to the values recorded in table 5.16, with TRolke uncertainties typically being roughly $10 \%$ smaller.

Figure 5.29 shows the tagging efficiency as a function of $\mathrm{qPE}$, showing that the Monte Carlo predicts a constant tagging efficiency as a function of qPE. This efficiency was calculated with ROOT's TEfficiency class, which assumes a binomial distribution. The total number of events (Bernoulli trials) is given by the total number of events in the $\mathrm{MC}$ dataset with $0.6<$ fprompt $<0.8$, qPE $>100$, and that pass standard pileup cuts. The number of binomial successes is given by the number of tagged pairs of ${ }^{214} \mathrm{Bi}$ and ${ }^{214} \mathrm{Po}$ decays within the $1.5 \mathrm{~ms}$ window. 


\begin{tabular}{|c|c|}
\hline Process or Cut & Efficiency \\
\hline Tagging & $0.648 \pm 0.052$ \\
sep $<200 \mathrm{~mm}$ & $0.556 \pm 0.053$ \\
fmaxpe $<0.2$ & $0.620 \pm 0.052$ \\
sep $<200$ mm and fmaxpe $<0.2$ & $0.528 \pm 0.053$ \\
\hline
\end{tabular}

Table 5.14: Efficiencies of cuts used to identify surface events with degraded reconstructed energy. These were found by a Monte Carlo study on ${ }^{214} \mathrm{Po}$ surface tags with $5000<$ qPE $<35000$ and $0.6<$ fprompt $<0.8$. Low Level, Pileup, and fmaxpe $<0.4$ cuts were applied before tagging. Both the numerator and denominator in the efficiency calculations used here include these cuts. The tagging efficiency is close to $50 \%$ because half of the $\beta$ events are lost to the TPB for surface ${ }^{214} \mathrm{Bi}$ events.

\begin{tabular}{|c|c|c|c|c|c|c|c|c|}
\hline qPE Range & \multicolumn{2}{|c|}{ Tagging Cuts } & \multicolumn{2}{c|}{ sep $<\mathbf{2 0 0} \mathbf{~ m m}$} & \multicolumn{2}{|c|}{ fmaxpe $<\mathbf{0 . 2}$} & \multicolumn{2}{c|}{ sep $<\mathbf{2 0 0}+$ fmaxpe $<\mathbf{0 . 2}$} \\
\hline & Signal & Random & Signal & Random & Signal & Random & Signal & Random \\
\hline $25,400-{ }^{214}$ Po peak & 145 & 80 & 55 & 0 & 140 & 78 & 52 & 0 \\
$14,000-25,400$ & 106 & 60 & 38 & 2 & 102 & 60 & 35 & 2 \\
$6,000-14,000$ & 47 & 17 & 31 & 2 & 46 & 17 & 30 & 2 \\
$500-6,000$ & 80 & 33 & 42 & 0 & 61 & 27 & 32 & 0 \\
$240-500$ & 26 & 9 & 16 & 1 & 10 & 7 & 5 & 1 \\
$100-240$ & 139 & 87 & 27 & 0 & 100 & 60 & 13 & 0 \\
$0-100$ & 16969 & 17365 & 206 & 208 & 9184 & 10197 & 112 & 127 \\
\hline
\end{tabular}

Table 5.15: Raw numbers of tags in signal and background window for various qPE ranges after cuts listed in each column heading. The "tagging cuts" - Low level, pileup, and fmaxpe $<0.4$ - were applied to both tagged events. 


\begin{tabular}{|c|c|c|c|c|}
\hline qPE Range & Low Level, Pileup, fmaxpe $<0.4$ & sep $<200 \mathrm{~mm}$ & fmaxpe $<0.2$ & sep $<200 \mathrm{~mm}$ and fmaxpe $<0.2$ \\
\hline $25,400-{ }^{214}$ Po peak & $100 \pm 24$ & $99 \pm 16$ & $100 \pm 25$ & $99 \pm 17$ \\
$14,000-25,400$ & $71 \pm 21$ & $65 \pm 13$ & $68 \pm 21$ & $63 \pm 13$ \\
$6,000-14,000$ & $46 \pm 13$ & $52 \pm 11$ & $47 \pm 13$ & $53 \pm 12$ \\
$500-6,000$ & $73 \pm 17$ & $76 \pm 14$ & $55 \pm 16$ & $61 \pm 12$ \\
$240-500$ & $26.2 \pm 9.4$ & $27.0 \pm 7.9$ & $4.8 \pm 6.7$ & $7.6 \pm 4.7$ \\
$100-240$ & $80 \pm 24$ & $49 \pm 10$ & $64 \pm 21$ & $24.6 \pm 7.3$ \\
$0-100$ & $-610 \pm 290$ & $-4 \pm 37$ & $-1630 \pm 260$ & $-28 \pm 29$ \\
\hline
\end{tabular}

Table 5.16: Surface candidates in various qPE ranges corrected for efficiencies. The efficiencies are listed in table 5.14. To obtain these numbers, the listed cuts were applied and a count obtained (table 5.15). The background was subtracted using the random window, and the result was scaled using the corresponding efficiency. Note that Low Level, Pileup, and fmaxpe $<0.4$ cuts were applied before tagging.

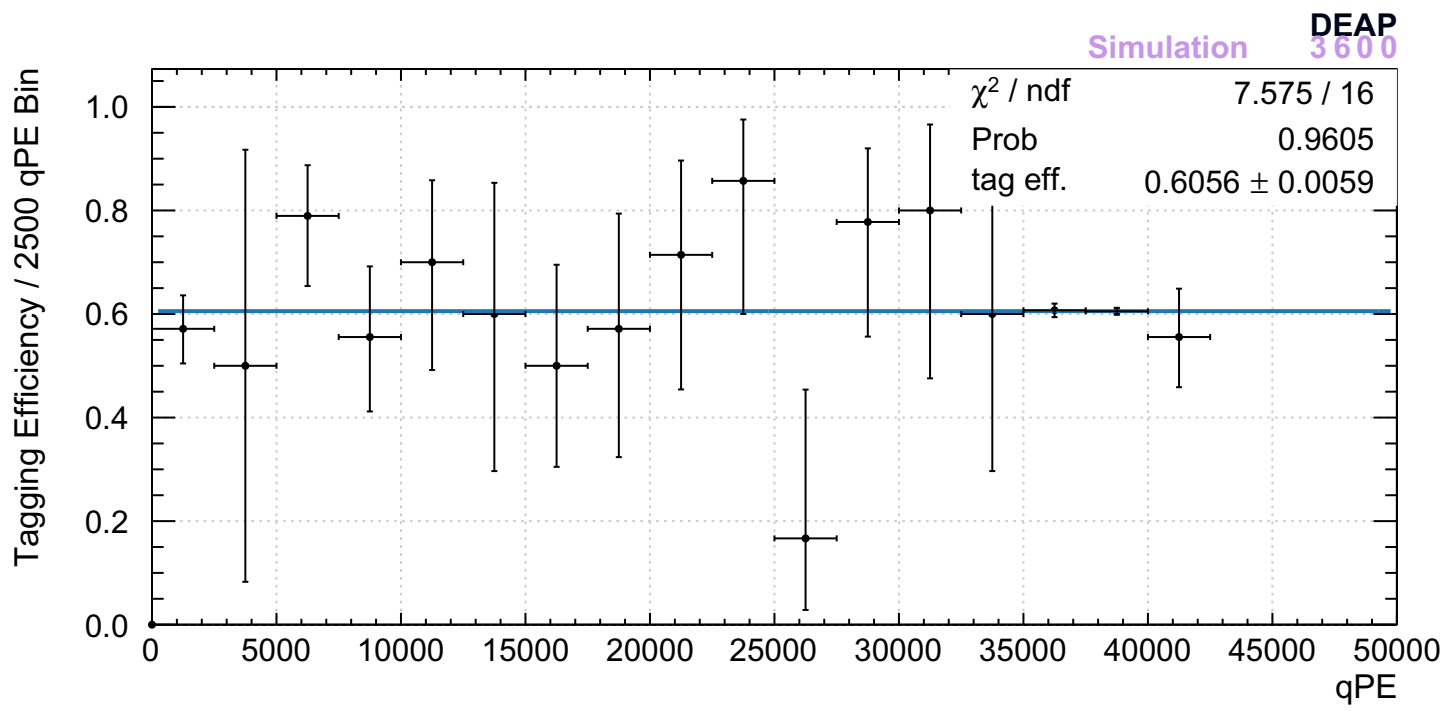

Figure 5.29: Monte Carlo tagging efficiency for $\mathrm{Bi}^{214} \mathrm{Po}$ events. This efficiency is for events with $0.6<$ fprompt $<0.8$, qPE $>100$, and that pass pileup cuts. This efficiency is close to $50 \%$ due to the fact that $50 \%$ of surface $\beta$ events will be directed towards the TPB. It was determined by dividing the number of tags by the number of events prior to tagging in each qPE bin. A horizontal line was fitted to the distribution. 


\section{Applied Cuts for Neck $\alpha$ Control Region}

\begin{tabular}{l|c|c}
\hline \hline Cut Variable & Cut String to Keep Events & Analysis Correction \\
\hline calcut & $(!($ calcut\&0x31f8) $)$ & Deadtime \\
dtmTrigSrc & $(!($ dtmTrigSrc\&0x82) $)$ & Deadtime \\
subeventN & $==1$ & Pileup \\
trigTime & $2350<$ trigTime $<2650$ & Efficiency \\
timefitT0 & $100<$ timefitT0 $<5500$ & Efficiency \\
fmaxpe & $<0.035$ & Efficiency \\
mblikelihoodz & $<700 . \mathrm{mm}$ & Efficiency \\
\hline
\end{tabular}

Table 5.17: List of cuts applied to data in order to identify neck $\alpha$ events.

\section{${ }^{214}$ Po Tags in the Neck}

The DEAP-3600 neck geometry directly above the LAr volume is shown in Figure 5.30. The flowguides serve to aid the flow of "warm" argon to the cooling coils, and cooled argon back down to the main detector volume. It is hypothesized that a small amount of liquid argon was present on the surfaces of the neck, making some level of scintillation possible for particles that enter it. The thinness of the liquid argon layer combined with the shadowing effect illustrated in Figure 5.30 can cause the reconstructed energy of $\alpha$ decays to be reduced down to the scale of WIMP interactions. It was, therefore, extremely important to understand this background as much as possible to exclude these events from the WIMP search and to quantify the expected number of events from the neck that would pass the exclusion cuts. For more details, see [1].

Since ${ }^{210} \mathrm{Po}$ makes up the bulk of the surface events in the liquid argon, it is assumed that they are the dominant source of surface events in the neck. To check this assumption, a ${ }^{214} \mathrm{Bi}-{ }^{214} \mathrm{Po}$ tagging analysis was done on the neck events. The neck events are selected using the cuts listed in table 5.17. Figure 5.32 shows what 


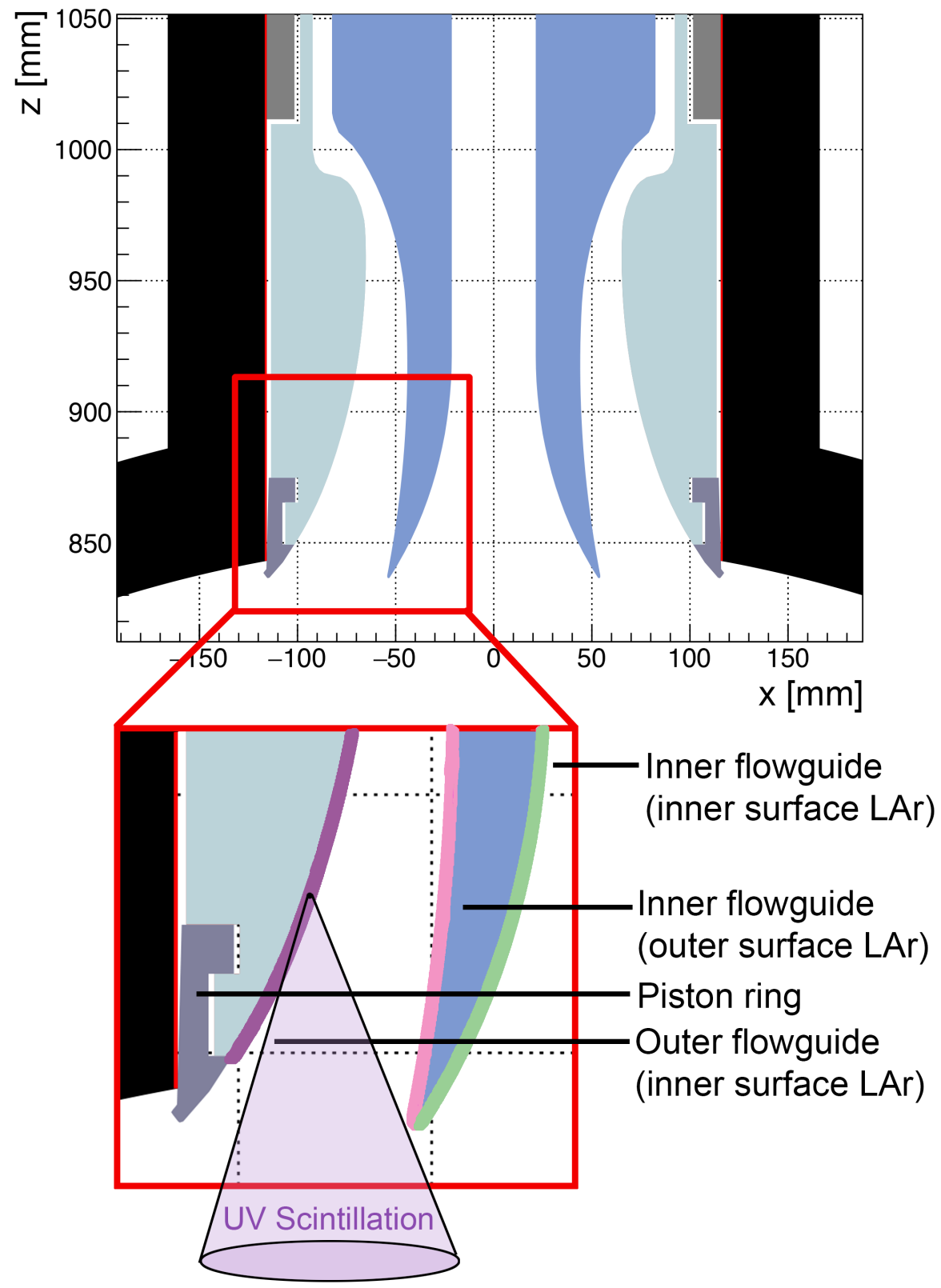

Figure 5.30: Diagram (from [1]) showing the flow guide geometry in the neck. 


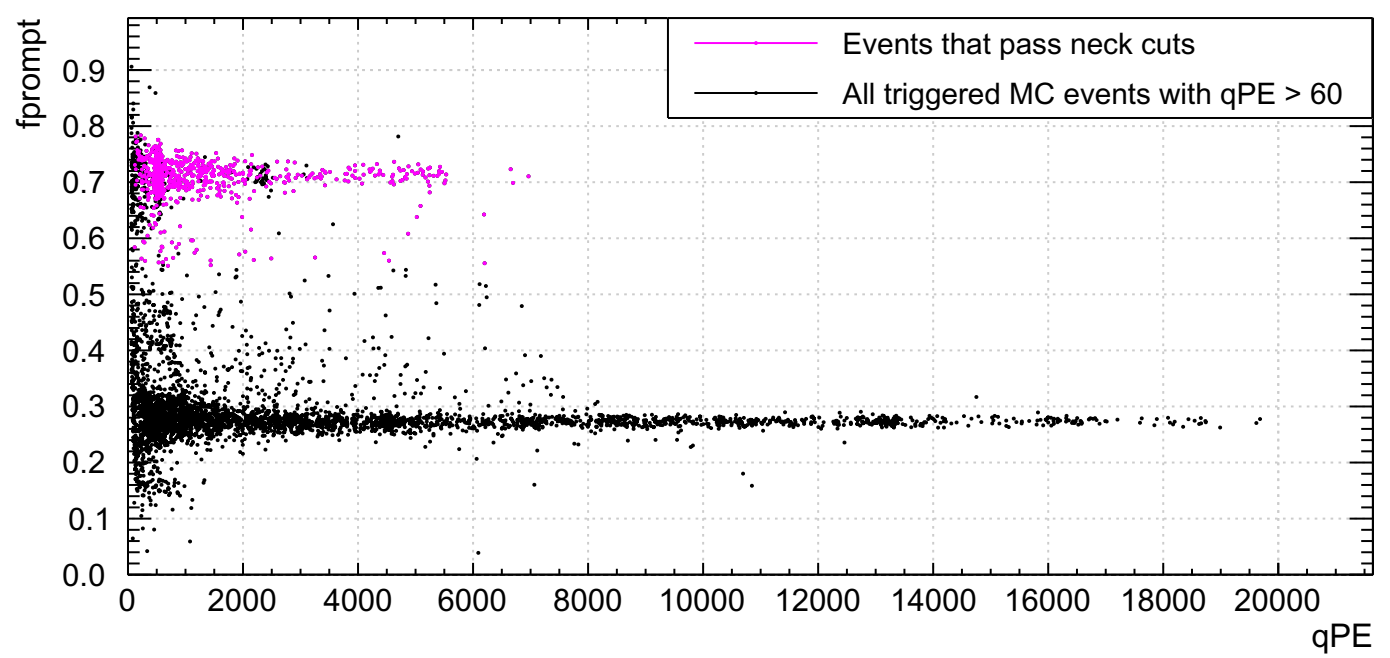

Figure 5.31: The fprompt vs qPE distribution of the simulated inner flowguide $\mathrm{Bi}^{214} \mathrm{Po}$ events. $14,900{ }^{214} \mathrm{Bi}$ decays were generated. These events were tagged and compared with data (see Figures 5.33 and 5.34). The purple events are those which pass the neck $\alpha$ selection cuts listed in table 5.17 .

these events look like in data.

\begin{tabular}{|l|l|l|l|}
\hline Dataset & $\begin{array}{l}\text { Number in } \\
\text { Dataset } \\
\text { (prior to } \\
\text { tagging) }\end{array}$ & $\begin{array}{l}\text { After } \\
\text { Tagging } \\
\text { (Signal } \\
\text { Window) }\end{array}$ & $\begin{array}{l}\text { Random } \\
\text { Window }\end{array}$ \\
\hline 247.17 Day Neck Data & 1315 & 9 & 3 \\
\hline $14,900{ }^{214}$ Po MC on IFG & 651 & 115 & 0 \\
\hline
\end{tabular}

Table 5.18: Numbers of events before and after tagging for neck events selected from the 247.17 day dataset and Monte Carlo $\mathrm{Bi}^{214} \mathrm{Po}$ decays on the inner flowguide. Both datasets are constrained in qPE: $60<\mathrm{qPE}<7000$.

After running the tagging algorithm, 12 events were identified which pass the neck $\alpha$ selection cuts. 9 of these events were in the BiPo signal window, and 3 were in the random window. In order to determine the tagging efficiency for neck $\mathrm{Bi}^{214} \mathrm{Po}$ events, a Monte Carlo simulation of $\mathrm{Bi}^{214} \mathrm{Po}$ events on the inner flowguide was run and $14,900{ }^{214} \mathrm{Bi}$ decays were generated (see Figure 5.31). These Monte Carlo events 


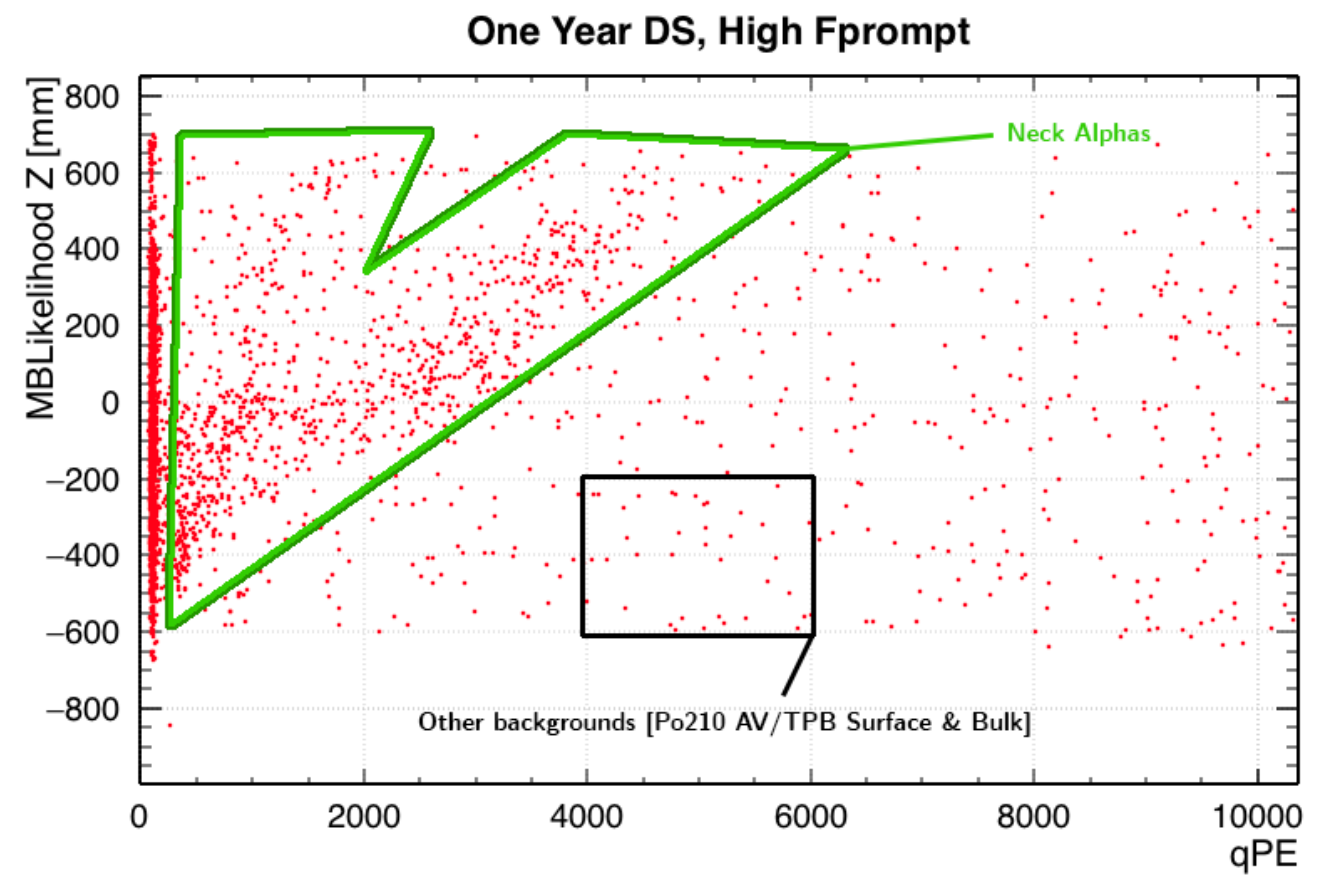

Figure 5.32: Shown is the distribution of events in the (mblikelihoodZ, qPE) plane from physics data passing cuts outlined in Table 5.17. The green region is the approximate location in this representation of ${ }^{210} \mathrm{Po} \alpha$-decay candidates from the neck region. The black region denotes background events not associated with ${ }^{210} \mathrm{Po} \alpha$-decays in the neck, and are likely leakage of ${ }^{210} \mathrm{Po}$ decays from the AV acrylic bulk and surface and TPB bulk.

were also tagged in the same way. The results of this study are shown in Figures 5.33 and 5.34 and in table 5.18 .

In conclusion, more statistics are needed to determine whether ${ }^{214} \mathrm{Po}$ is present among these $\alpha$ events. Only $0.77 \%$ of events generated in the Monte Carlo were ultimately tagged, and $18 \%$ of triggers were tagged (see table 5.18). Using the TRolke class in ROOT (which implements $|58|$ ), we get an upper limit of $68{ }^{214}$ Po triggers (90\% CL) in the neck in the 247.17-day dataset. 


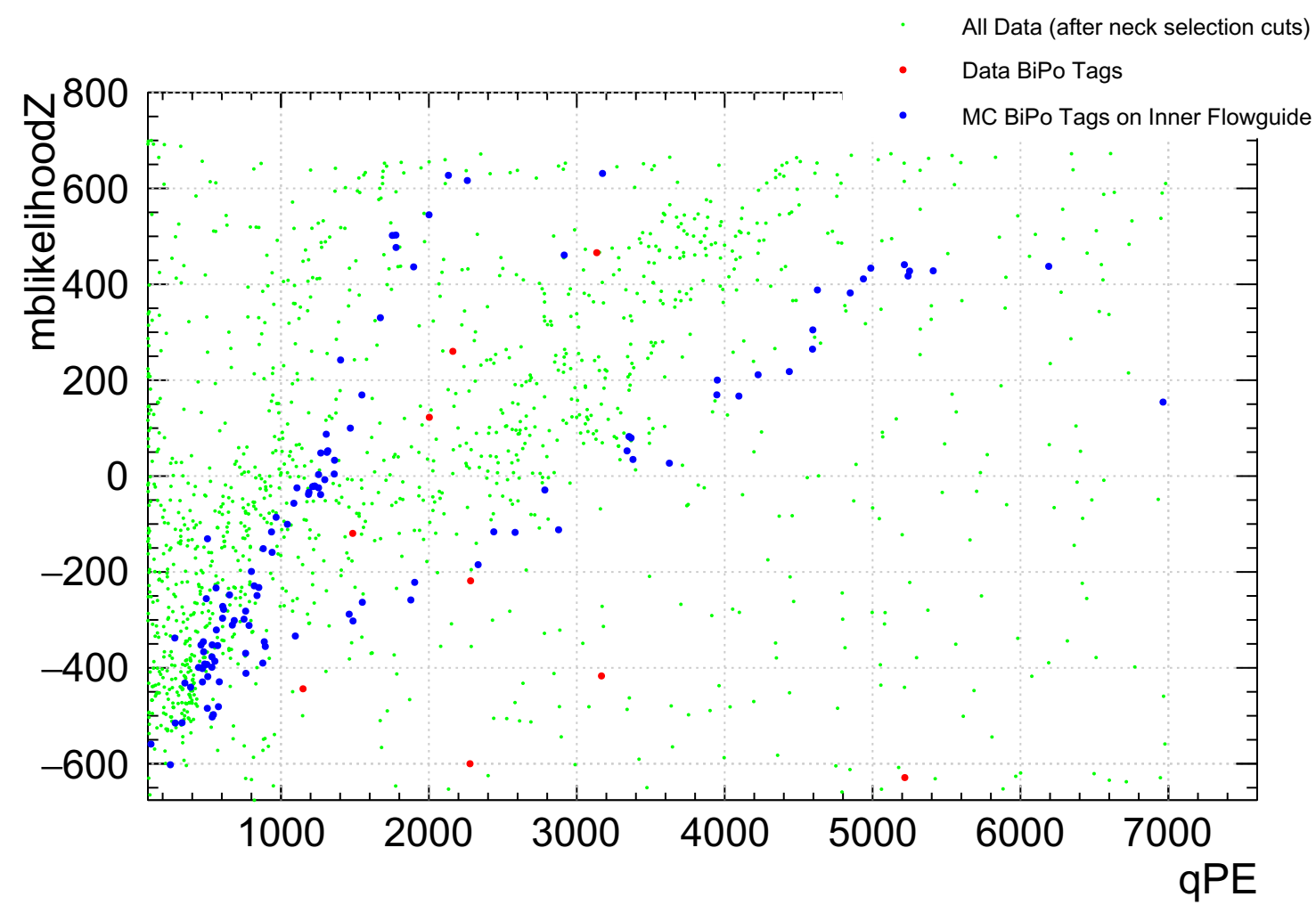

Figure 5.33: The distribution of data and Monte Carlo neck events in mblikelihoodZ vs qPE space. The Monte Carlo events were generated on the inner flowguide. $14,900{ }^{214} \mathrm{Bi}$ decays were generated.The green events represent the data after applying the cuts in table 5.17 (these are the same as the red events in Figure 5.32). The red events are events that have been tagged with a low fprompt $(0.2<$ fprompt $<0.4)$ and high $\mathrm{qPE}(\mathrm{qPE}>4000)$ event, which is the region where we expect to find ${ }^{214} \mathrm{Bi}$ decay $\gamma$ and $\beta$ events.

\section{Low fmaxpe Degraded ${ }^{214}$ Po Tags}

There is evidence that there may be a small amount of dust in the liquid argon. The main motivation for this hypothesis is the observation of events with qPE values lower than those of events in the full energy peaks and with an fmaxpe value lower than would be expected from Monte Carlo studies of surface events. One explanation for the existence of these events is that decays of radioactive isotopes located in dust particles in the liquid argon cause these events to have degraded reconstructed energies (due to energy deposited in the dust particle rather than the LAr) and a low 


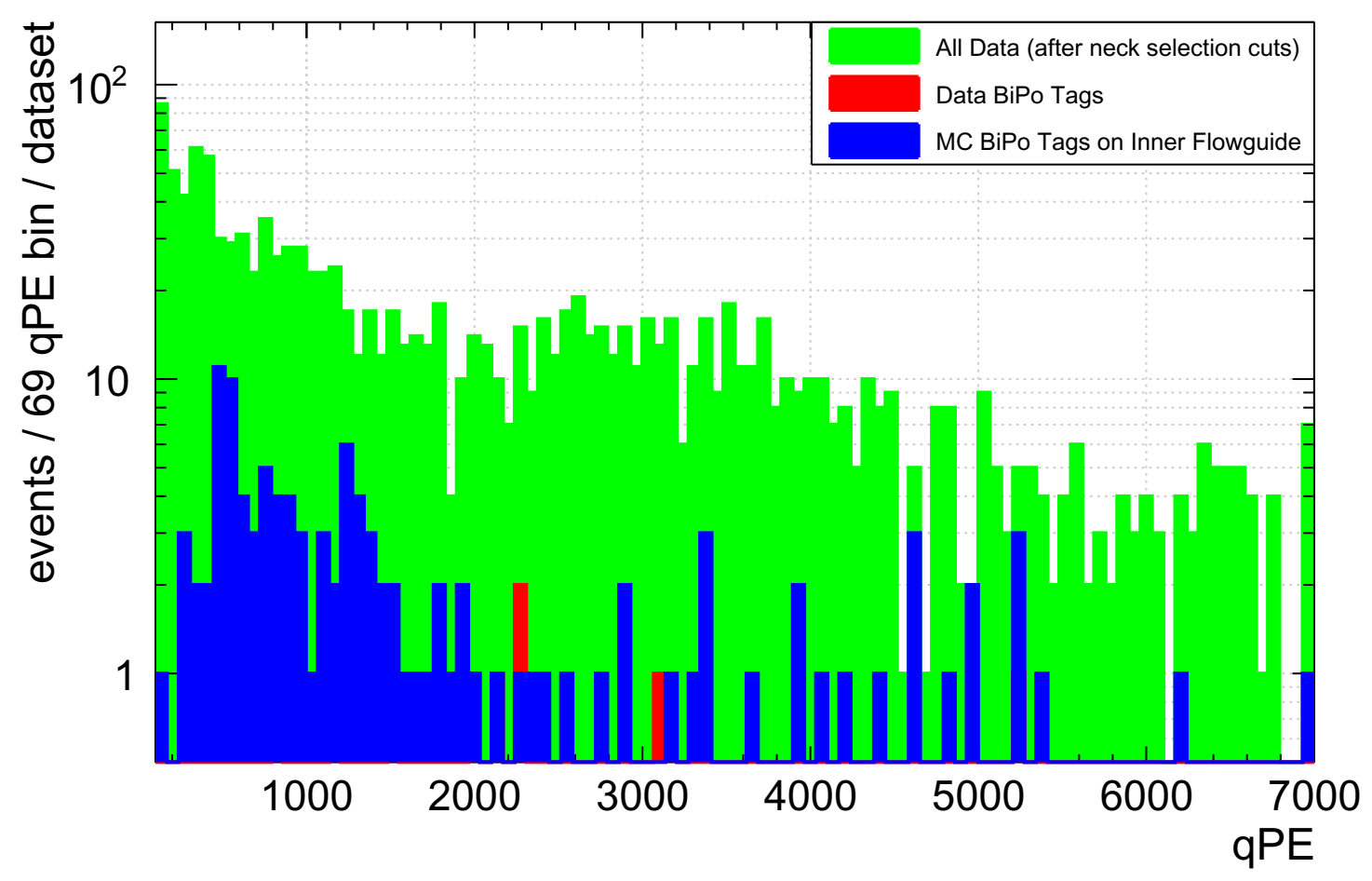

Figure 5.34: The qPE distribution of data and Monte Carlo neck events. 14,900 Monte Carlo events were generated on the inner flowguide. The green events represent the data after applying the cuts in table 5.17 (these are the same as the red events in Figure 5.32. The red events are events that have been tagged with a low fprompt $(0.2<$ fprompt $<0.4)$ and high $\mathrm{qPE}(\mathrm{qPE}>4000)$ event.

fmaxpe value. We can check whether these dust-like events include any ${ }^{214} \mathrm{Po}$ using this tagging analysis. These events typically have an fmaxpe value below 0.035. In Figure 5.35, these events are those with fmaxpe below the main ${ }^{210}$ Po peak (around 22,500 qPE). The 3-4 distributions at higher qPE are other $\alpha$ populations. The one at the highest qPE range $(35,000-45,000)$ is ${ }^{214} \mathrm{Po}$.

These events also tend to reconstruct at higher fprompt than would be expected from surface events. This is shown in Figure 5.36. The events above approximately 27,000 qPE are mostly liquid argon bulk decays, while those below 25,000 are mostly ${ }^{210} \mathrm{Po}$ surface decays. The events with fprompt above the ${ }^{210} \mathrm{Po}$ peak are the ones under discussion here. 


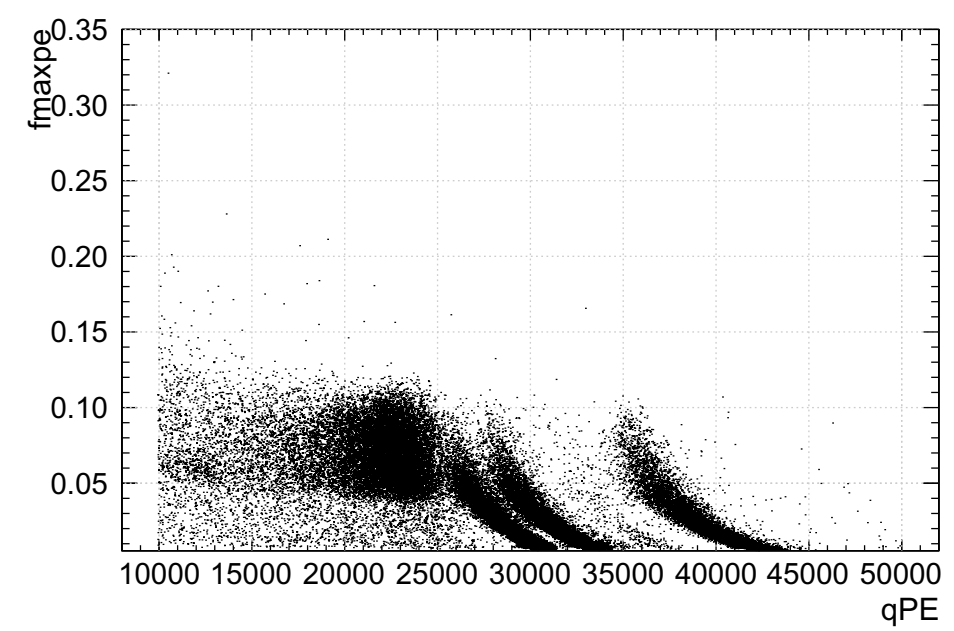

Figure 5.35: fmaxpe vs qPE for data. The dust-like events are those with an fmaxpe less than 0.035 . The distribution above this is surface ${ }^{210}$ Po. Monte Carlo studies have shown that surface events should not produce a population of events with fmaxpe less than 0.035. Standard low-level and pileup cuts were applied.

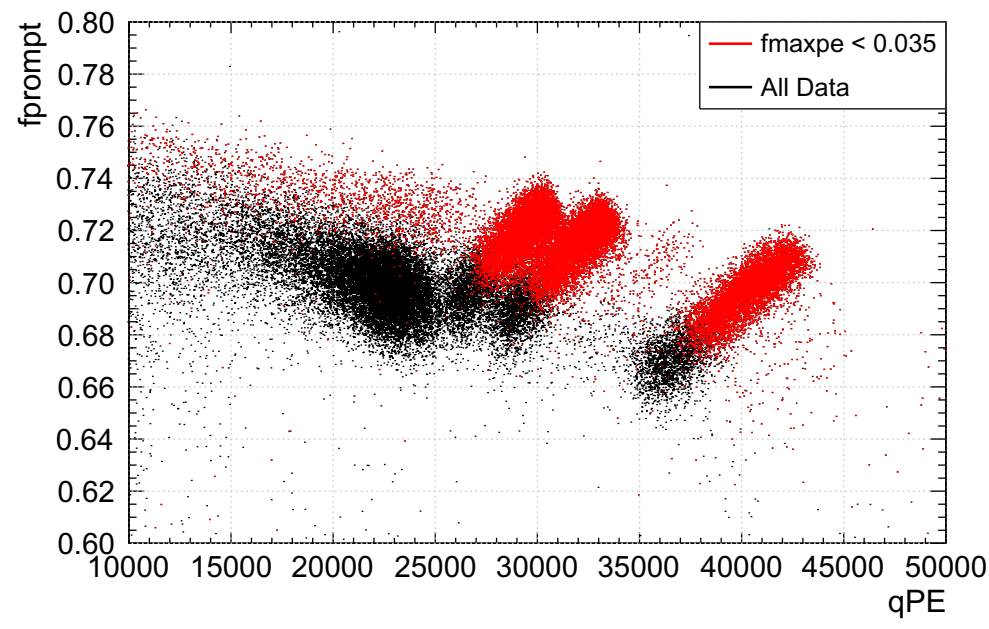

Figure 5.36: Distribution of data events in fprompt vs qPE space. The events with fmaxpe $<0.035$ (red) are overlaid with the rest of the data (black). 

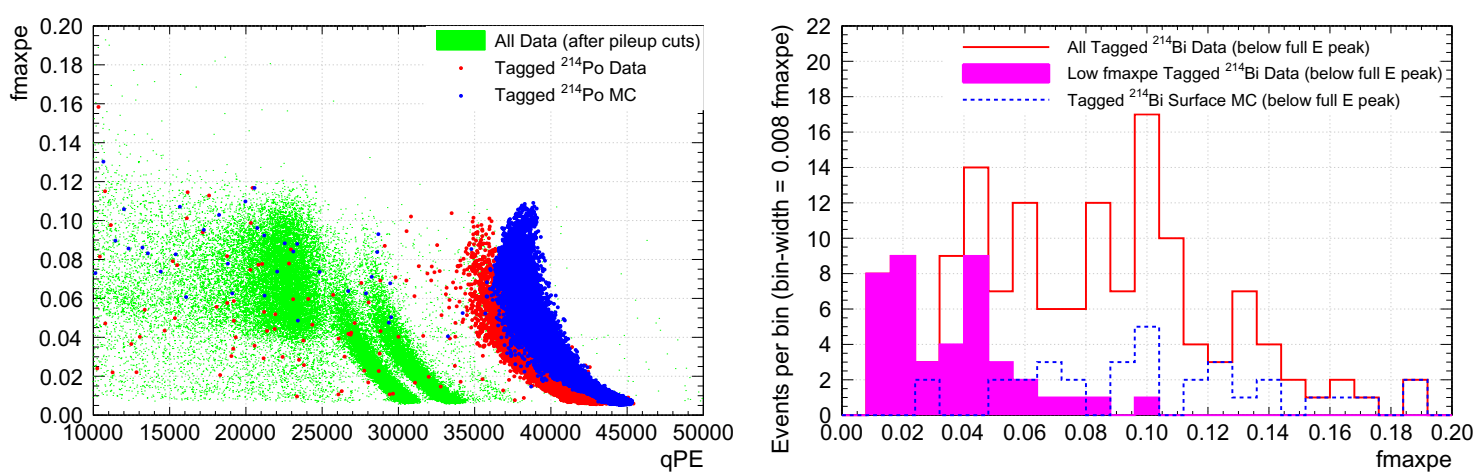

Figure 5.37: Left: fmaxpe vs qPE distribution of tagged ${ }^{214} \mathrm{Po}$ high fprompt data events (red) and simulated ${ }^{214}$ Po surface and LAr bulk tags (blue) overlaid with the rest of the data (green). There are no simulated surface or LAr bulk events with fmaxpe $<0.04$ at energies below the main ${ }^{214}$ Po surface peak. Right: fmaxpe distribution of tagged ${ }^{214} \mathrm{Bi}$ data events (red line), filled according to the number of events in which the corresponding ${ }^{214}$ Po tag had fmaxpe $<0.035$, and overlaid with the surface $\mathrm{MC}$ distribution of ${ }^{214} \mathrm{Bi}$ tags. Only events below the full energy peaks were selected when making the plot on the right. Standard low-level and pileup cuts were applied to events in both plots, in addition to the requirement that the tagged pairs be within $200 \mathrm{~mm}$ of each other.

$\mathrm{Bi}^{214}$ Po tagging is one method that we can use to help determine whether these are $\alpha$ events. As can be seen in Figure 5.20, a few of the ${ }^{214}$ Po tags with degraded reconstructed energies (surface candidates) have an fprompt in the same region as the low fmaxpe events we are studying. And on the left portion of Figure 5.38 is shown that some of these tags also have fmaxpe values lower than expected from Monte Carlo studies of surface ${ }^{214}$ Po events. Note that only 2 events appear in the random window for the selection cuts used to make Figure 5.37, making it unlikely that these events are random coincidence events.

The fmaxpe variable is closely correlated with the reconstructed radial position. This is shown in figure 5.38. The reconstructed radial positions of the low fmaxpe degraded $\alpha$ tags follow the same relation with fmaxpe as the rest of the data.

The locations of these events projected onto a 2D map of the surface of the detector are shown in Figure 5.39. Though the statistics are low, there seems to be a small 

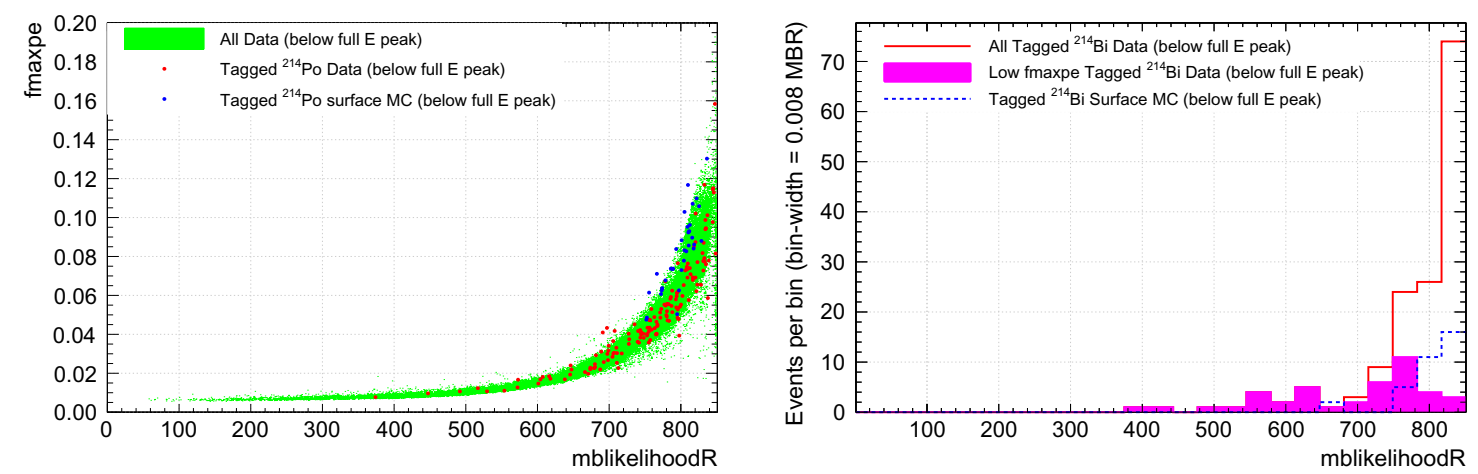

Figure 5.38: Left: fmaxpe vs MBR distribution of tagged ${ }^{214}$ Po data events (red) and simulated ${ }^{214} \mathrm{Po}$ surface tags (blue) overlaid with the rest of the data (green). This plot excludes events in the full energy ${ }^{214}$ Po peak to show only ${ }^{214}$ Po candidates with degraded reconstructed energy. Right: mblikelihoodR distribution of ${ }^{214} \mathrm{Bi}$ tags in data (red line), filled according to the number of events in which the corresponding ${ }^{214} \mathrm{Po}$ tag had fmaxpe $<0.035$, overlaid with the surface MC distribution of ${ }^{214} \mathrm{Bi}$ tags. Standard low-level and pileup cuts were applied to events in both plots, in addition to the requirement that the tagged pairs be within $200 \mathrm{~mm}$ of each other.

preference for the bottom of the detector, similar to typical surface events. This fits the hypothesis of dust in the LAr, since it is unlikely that a contaminant such as dust would have the same density as liquid argon. The PMT hit maps for low fmaxpe events in which both events in a tag are required to have fmaxpe $<0.035$ are shown in Figure 5.40. To select these events, standard low level and pileup cuts were applied, the tag separation was constrained to $200 \mathrm{~mm}$ or less, the fmaxpe of both events in each tag was constrained to less than 0.035, and it was required that 2000 $<\mathrm{qPE}<27000$. The charge distribution for these events is much broader than what is expected from typical surface events (Figure 5.41). This is strong evidence that these are not normal surface events.

In conclusion, the hit maps of these tagged low fmaxpe ${ }^{214}$ Po candidates don't seem consistent with surface events. The fitted lifetime of all degraded ${ }^{214}$ Po candidates tagged with low fprompt events is consistent with that of ${ }^{214}$ Po. While this fits the hypothesis of dust in the liquid argon quite well, dust may not be the only possible 


\section{Relative Efficiency vs Cosine Theta and Phi}

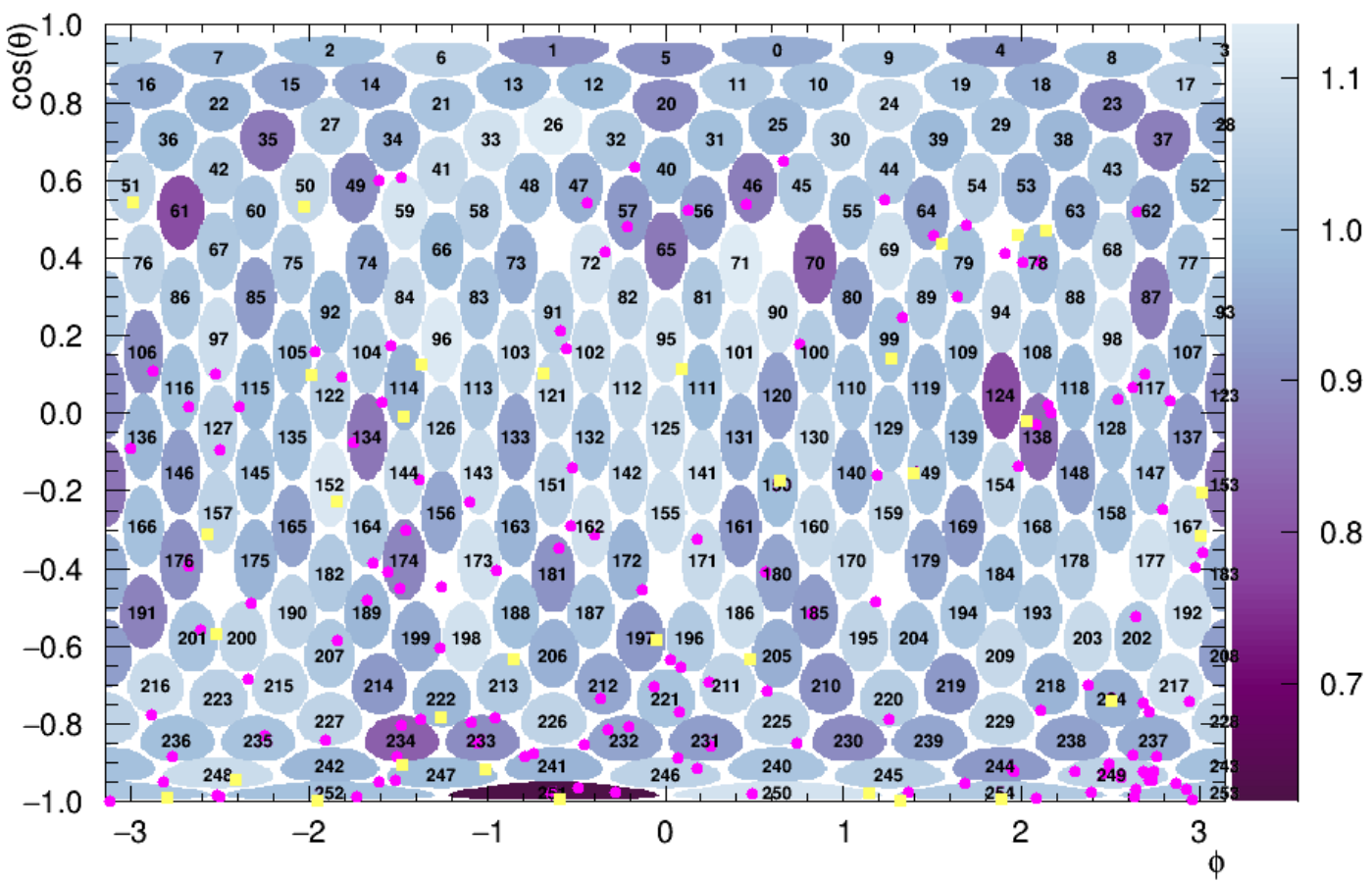

Figure 5.39: Same as Figure 5.28 showing the locations of the tagged ${ }^{214}$ Po surface candidates in purple, but with the locations of the low fmaxpe degraded ${ }^{214} \mathrm{Po}$ tags overlaid in yellow. All events are data events. 
explanation. A model to explain these events would require:

- A distribution of high fprompt events from $\alpha$ energy scales down to a few thousand $\mathrm{qPE}$

- A high energy low fprompt event followed by a high energy high fprompt event with a lifetime close to the ${ }^{214}$ Po lifetime

- A charge signature that is much broader than typical surface events

\subsubsection{Tagging ${ }^{220} \mathrm{Rn}$ and ${ }^{216} \mathrm{Po}$}

Time coincidence tagging is particularly useful for looking for ${ }^{216} \mathrm{Po}$ and its parent, ${ }^{220} \mathrm{Rn}$, as these have a factor of $30-40$ lower rate than ${ }^{220} \mathrm{Rn}$ chain events. Tagging is possible in this case due to the $145 \mathrm{~ms}$ half-life of ${ }^{216} \mathrm{Po}$. A window size of $0.5 \mathrm{~s}$ was used. The fprompt and qPE of the tags are plotted in Figure 5.42. Due to the time window size, the tagging algorithm is expected to miss $9 \%$ of ${ }^{216}$ Po decays. The resulting number of events in the dataset, after correcting for the events missed by the time window, is listed in table 5.5 .

To reduce the number of random coincidences, a spatial separation cut was used. As in the $\mathrm{Bi} /{ }^{216} \mathrm{Po}$ analysis, this was set to exclude tagged pairs that were separated by more than $200 \mathrm{~mm}$. This cut was based on Figure 5.43 (left), where it can be seen that all events in which the ${ }^{216} \mathrm{Po}$ candidate is within the ${ }^{216} \mathrm{Po}$ "box" in qPE and fprompt space are separated by less than $200 \mathrm{~mm}$.

A small number of neck-like events was also observed. These events were selected based on the amount of qPE observed in the top two rings of PMTs. The cut is shown in Figure 5.43 (right). As shown in Figure 5.43, a few tagged pairs exist at energies above the ${ }^{220} \mathrm{Rn} /{ }^{216} \mathrm{Po}$ regions. These may be related to detector "burst" events, which were analysed by the author and other collaborators, but are beyond the scope of this report. 
The time between tags was fit to check whether the sample is consistent with the hypothesis that it is primarily made up of ${ }^{220} \mathrm{Rn} /{ }^{216} \mathrm{Po}$ tags. The fit was done both with and without the $200 \mathrm{~mm}$ separation cut. The fits are shown in Figure 5.44. A $2 \mathrm{~s}$ window was selected here to give a better fit of the random coincidence $/{ }^{222} \mathrm{Rn}$ ${ }^{218} \mathrm{Po}$ background. The fitted lifetimes of $0.217 \pm 0.024 \mathrm{~s}$ and $0.207 \pm 0.018 \mathrm{~s}$ are compatible with the expected ${ }^{216}$ Po lifetime of $0.209 \pm 0.003$ s. From the event count in table 5.5. $79^{218}$ Po events are expected to contribute to the background event count without the separation cut (Figure 5.44, left). There are a total of 50,633 events in the tagging search region after standard low-level and pileup cuts in the 247.17-day data-set, yielding a random-coincidence expectation of 240 events. The combined background expectation is 319 events, which is compatible with the background fit result (without the separation cut) of $291 \pm 29$ events. The decay amplitude is also relatively unchanged between the left and right plots, indicating that, as expected from Figure 5.43 , the spatial separation cut does not remove a significant number of ${ }^{220} \mathrm{Rn} /{ }^{216} \mathrm{Po}$ tags.

Events with degraded reconstructed energy (relative to the energy of $\alpha$ decays in the LAr bulk) were selected by constraining the ${ }^{216}$ Po tag to lie outside the ${ }^{216}$ Po "box" in qPE and fprompt space, while keeping the $200 \mathrm{~mm}$ spatial separation requirement. The reconstructed locations of these events are shown in Figure 5.45 (left). As would be expected, neck-like events (based on the cut from Figure 5.43 , right) are shown to reconstruct close to the neck, while low fmaxpe (dust-like) events reconstruct closer to the centre of the detector. To test the hypothesis that these are predominantly ${ }^{220} \mathrm{Rn} /{ }^{216} \mathrm{Po}$ decays, the time between them was fit. The fitted lifetime of $0.211 \pm$ $0.074 \mathrm{~s}$ is compatible with the expected ${ }^{216} \mathrm{Po}$ lifetime of $0.209 \pm 0.003 \mathrm{~s}$.

In conclusion, this analysis found $349 \pm 23{ }^{220} \mathrm{Rn} /{ }^{216} \mathrm{Po}$ pairs between 10,000 and $50,000 \mathrm{qPE}$ in the 247.17 -day dataset. There is also evidence for ${ }^{220} \mathrm{Rn} /{ }^{216} \mathrm{Po}$ dust-like events within the dataset. 


\subsubsection{Tagging ${ }^{222} \mathrm{Rn}$ and ${ }^{218} \mathrm{Po}$}

Though possible, time coincidence tagging is less useful for the analysis of ${ }^{222} \mathrm{Rn}$ and

${ }^{218} \mathrm{Po}$, due to the $3.1 \mathrm{~min}$ half-life of ${ }^{218} \mathrm{Po}$. No tagging study was therefore performed on this pair. Its rate was constrained solely by a fit of the $\alpha$ energy spectrum, as described in section 5.2 .

\subsection{Conclusion}

Several studies were performed to understand the distribution of $\alpha$ radioactivity in the DEAP-3600 detector. To quantify the activity of several $\alpha$-decaying isotopes in the LAr, a global fit was performed. This involved an event-by-event energy correction to remove the effects of PMT non-linearity and digitizer clipping. The results of this fit were published in [1]. Separate measurements were made for ${ }^{212} \mathrm{Po}$, since the very short lifetime of its daughter $\left({ }^{212} \mathrm{Bi}\right)$ results in the ${ }^{212} \mathrm{Po}$ decay piling up with the decay of its daughter.

Time-coincidence tagging was used to identify ${ }^{214} \mathrm{Po} \alpha$ decays where not all of the $\alpha$ energy was deposited in the LAr. An important application of this was to quantify the activity of ${ }^{214} \mathrm{Po}$ on the detector surface and to estimate the number of surface ${ }^{214}$ Po decays within various reconstructed energy ranges. This provided an estimate of ${ }^{214}$ Po within the WIMP ROI, allowing cuts to be optimized to remove them. It was also possible to identify evidence of ${ }^{214} \mathrm{Po}$ decays within dust particles in the LAr and to set a limit on ${ }^{214}$ Po decays within the surface LAr layer in the neck.

Time-coincidence tagging was used to identify ${ }^{220} \mathrm{Rn}$ and ${ }^{216} \mathrm{Po} \alpha$ decays, which also showed evidence of these isotopes decaying within dust in the LAr. It was also used to identify populations of these $\alpha$ decays for use in the energy correction used for the fit.

The evidence found for $\alpha$ on dust includes: 
- The preference of surface events to be located at the bottom of the detector

- The consistency of the lifetimes of tagged pairs of dust candidate events with known $\alpha$ populations

- The presence of $\alpha$-like events far from the inner surface with degraded reconstructed energy

- The inconsistency of these events with neck events

- The fact that these events are distributed throughout the LAr bulk, rather than being located near a few defective PMTs

- PMT hit maps of dust candidates that show light broadly distributed among the PMTs, which is inconsistent with the typical surface event

- Consistency of fmaxpe with reconstructed event position, indicating that the positions of these events are likely not being mis-reconstructed

These studies contributed to the best reported upper limit on the WIMP-nucleon spin-independent cross section on a LAr target of $3.9 \times 10^{-45} \mathrm{~cm}^{2}$ for a $100 \mathrm{GeV} / \mathrm{c}^{2}$ WIMP mass at 90\% CL [1]. 
Hits vs Cosine Theta and Phi

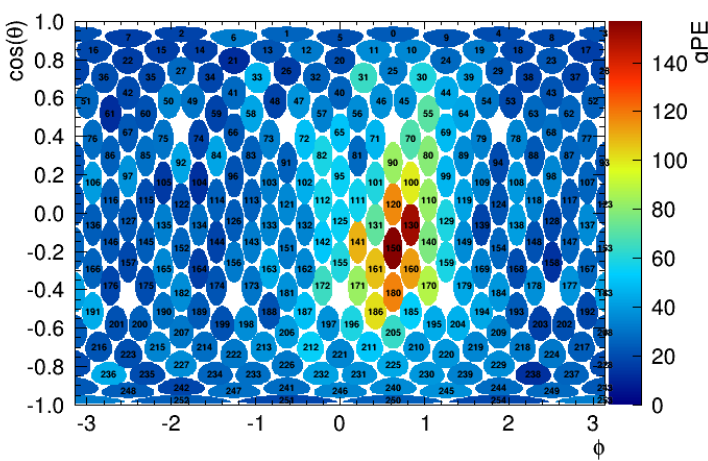

Hits vs Cosine Theta and Phi

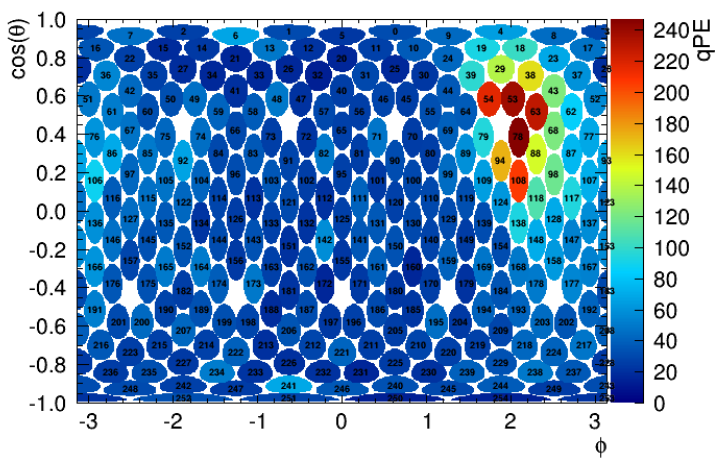

Hits vs Cosine Theta and Phi

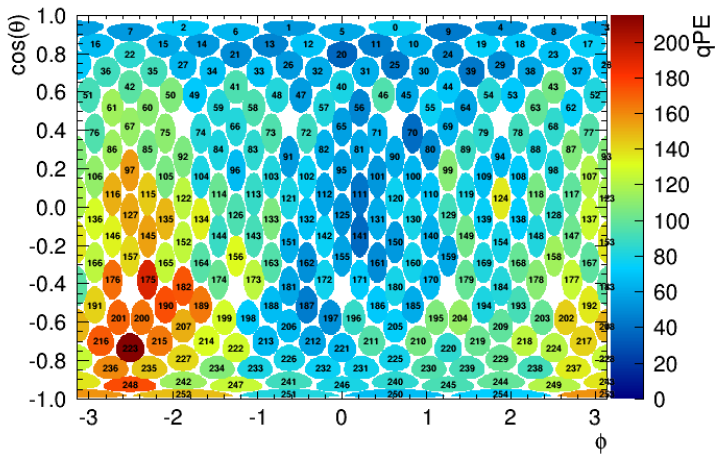

Hits vs Cosine Theta and Phi

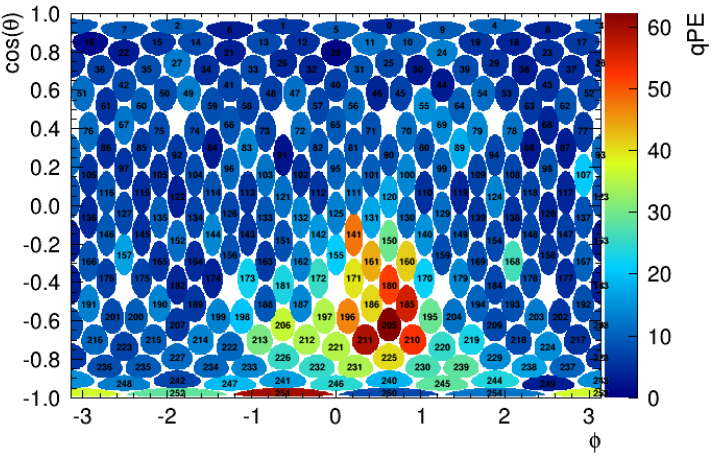

Hits vs Cosine Theta and Phi

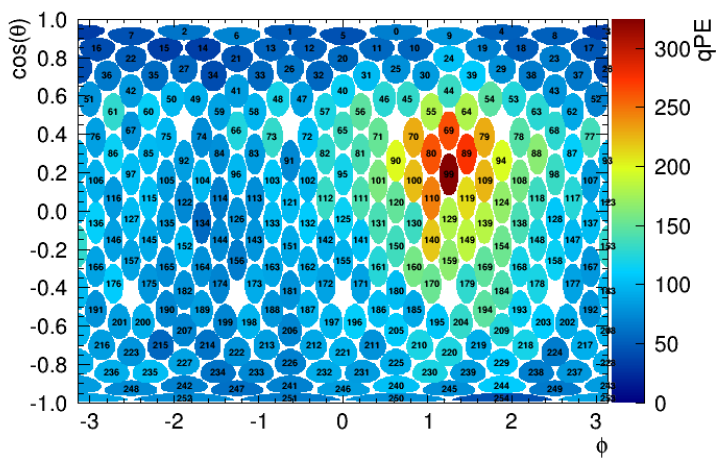

Hits vs Cosine Theta and Phi

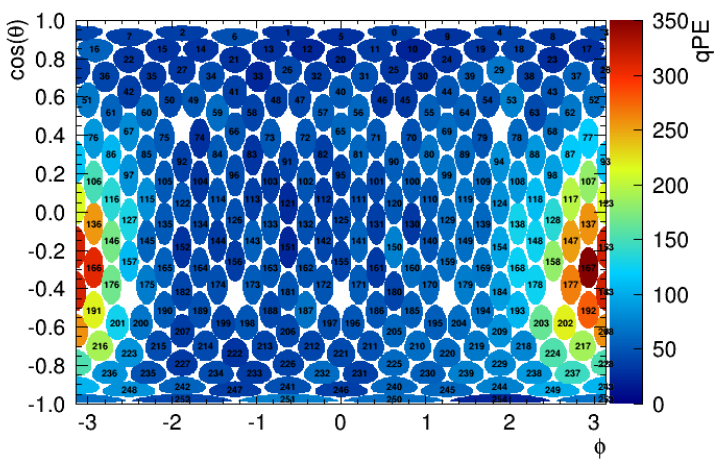

Hits vs Cosine Theta and Phi

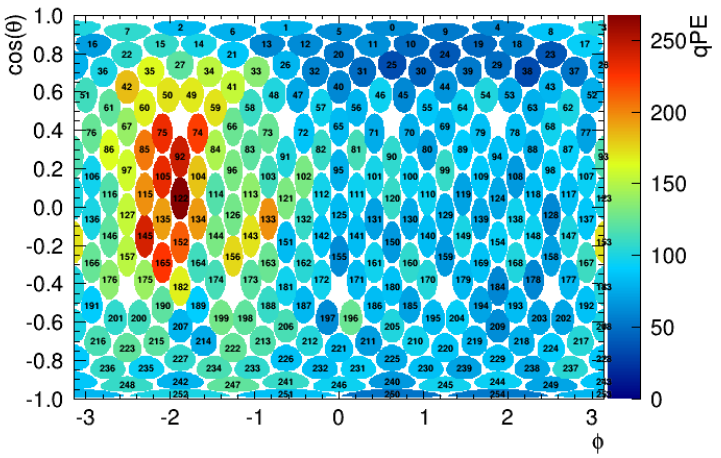

Figure 5.40: PMT hit maps of low fmaxpe ${ }^{214} \mathrm{Po}$ data tags. The numbers are PMT IDs, and the colour scale gives the reconstructed number of PE on each PMT. 

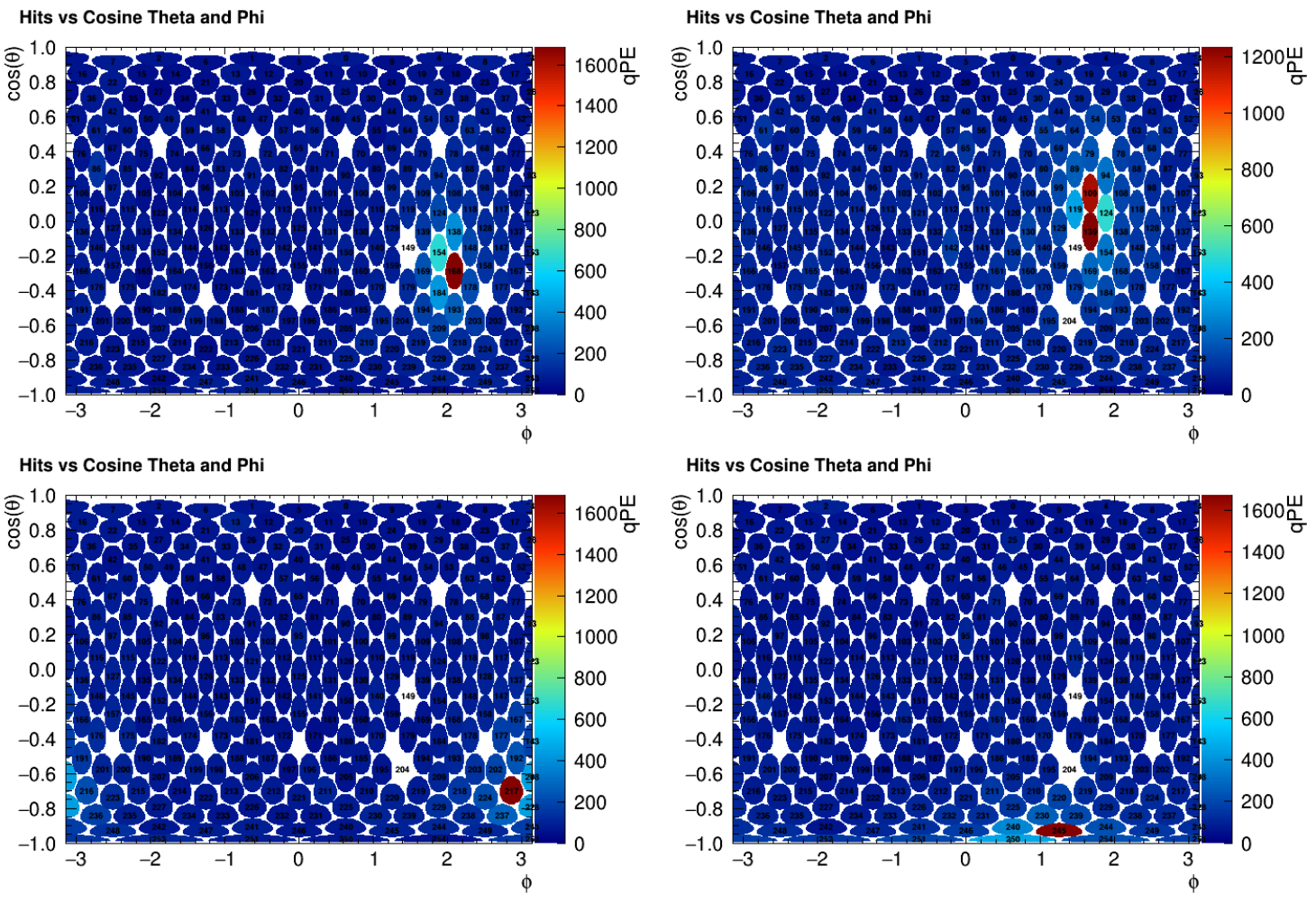

Figure 5.41: Sample PMT hit maps of ${ }^{210}$ Po surface events in data. These events were taken from the primary ${ }^{210} \mathrm{Po}$ surface feature. A lower fmaxpe bound of 0.04 was used in selecting these events.
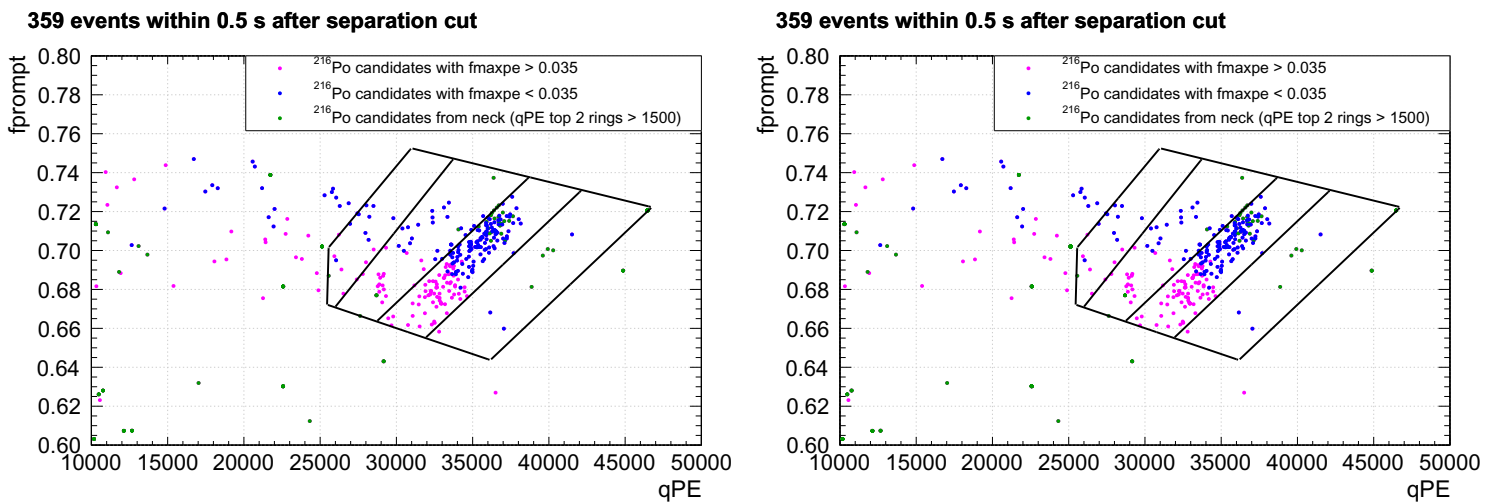

Figure 5.42: $\alpha$ - $\alpha$ tags in the data within a $0.5 \mathrm{~s}$ window with a spatial separation cut that excludes tagged pairs over $200 \mathrm{~mm}$ apart to reduce the level of random coincidences. Events with fmaxpe $<0.035$ are incompatible with surface $\alpha$ decay simulation, and may be from decays within dust particles. Neck events likely include both $\alpha$ decays and correlated event bursts that are unrelated to radioactivity. These events are selected from the cut shown in Figure 5.43. The boxes that overlay the events are (from left to right), ${ }^{222} \mathrm{Rn},{ }^{220} \mathrm{Rn} /{ }^{218} \mathrm{Po},{ }^{216} \mathrm{Po}$, and ${ }^{214} \mathrm{Po}$. 

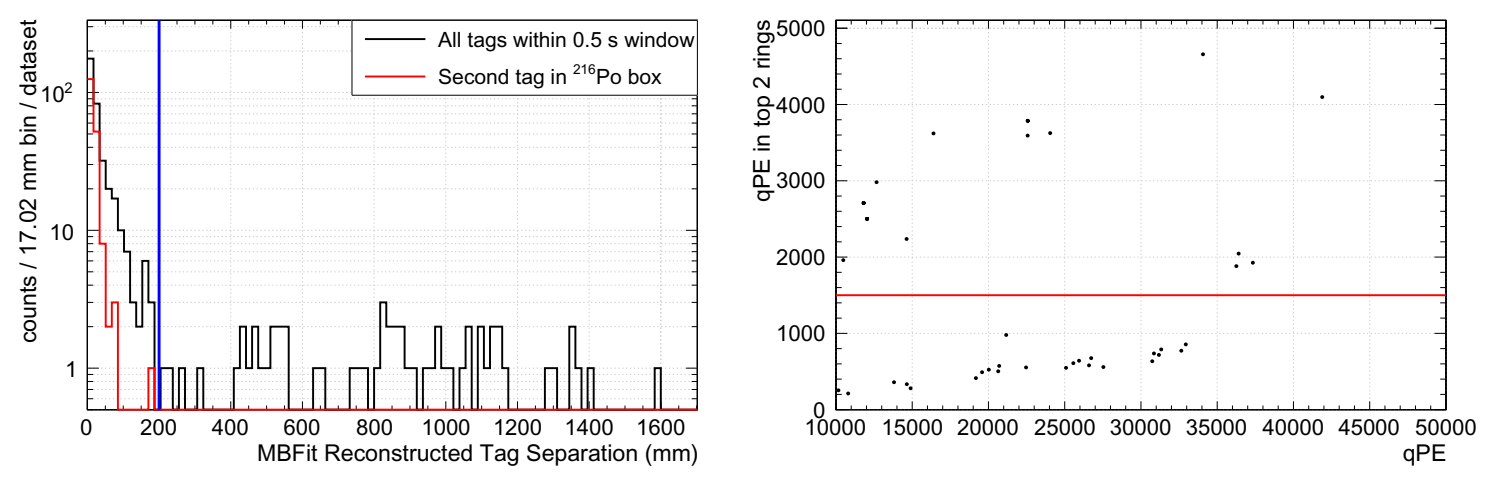

Figure 5.43: Left: The spatial separation of $\alpha$ - $\alpha$ data tags within a $0.5 \mathrm{~s}$ window. The red curve shows the spatial separation for the subset of events where the ${ }^{216} \mathrm{Po}$ candidate lies within the ${ }^{216} \mathrm{Po}$ "box" in qPE and fprompt space, showing that all such events that were found occurred within $200 \mathrm{~mm}$ of each other. Right: The qPE in the top 2 rings of PMTs (near the neck) vs the total event qPE for events where the ${ }^{216}$ Po candidate lies outside the ${ }^{216}$ Po candidate "box" in $\mathrm{qPE}$ and fprompt space. The red line shows the location of the neck cut that was used in this analysis. A linear relationship can be seen among events below this line, which is very different from the distribution above the line.
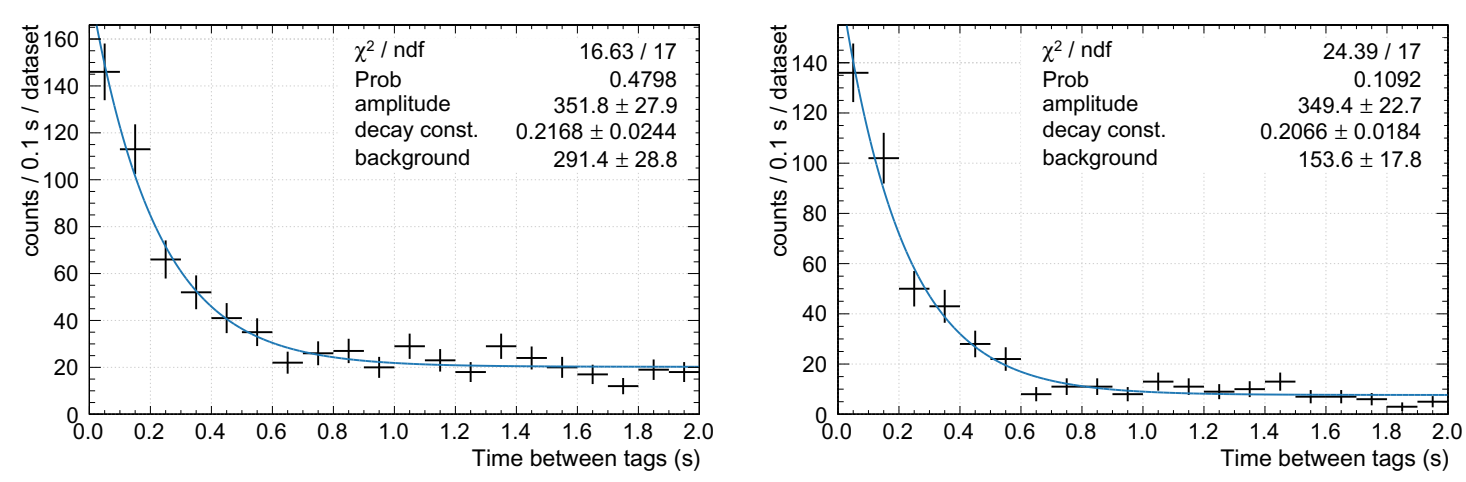

Figure 5.44: Fits to the time between $\alpha$ - $\alpha$ data tags. The amplitudes given in the fit results box show the estimated number of ${ }^{220} \mathrm{Rn} /{ }^{216} \mathrm{Po}$ decays and events in the background, and the fitted decay lifetime in seconds. Left: The fit without the $200 \mathrm{~mm}$ spatial separation cut. Right: The fit with the $200 \mathrm{~mm}$ spatial separation cut. 

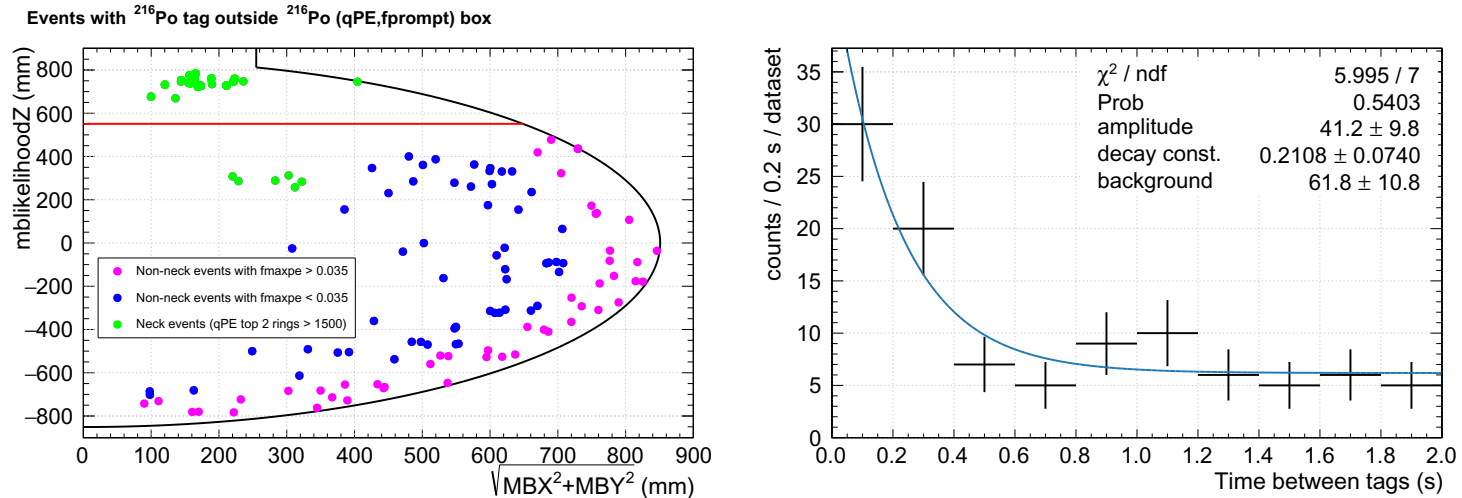

Figure 5.45: Left: Locations of $\alpha$ - $\alpha$ data tags $\left({ }^{220} \mathrm{Rn}\right.$ candidates) in which the ${ }^{216} \mathrm{Po}$ candidate is constrained to be outside the ${ }^{216} \mathrm{Po}$ "box" in qPE and fprompt space. These are, therefore, events with degraded reconstructed energy. Events with fmaxpe $<0.035$ are incompatible with surface $\alpha$ decay simulation, and may be from decays within dust particles. The neck events were selected according to the cut shown in Figure 5.43 . The detector surface is drawn as a solid black line, and the LAr fill level is shown as a solid red horizontal line. Right: A fit to the time between the non-neck-like tags in which the ${ }^{216} \mathrm{Po}$ candidate's reconstructed energy lies outside the ${ }^{216} \mathrm{Po}$ "box"and with fmaxpe $<0.035$. 


\section{Chapter 6}

\section{Estimation of the Radiogenic Neutron Background}

\subsection{Tagging Method}

The time coincidence tagging method that was used in the previous chapter to tag $\alpha$ decays was run on the open data as part of the analysis effort toward the upcoming third DEAP-3600 WIMP search result.

The process being searched for by the tagging method is illustrated in Figure 6.1 . A neutron is produced as the result of radioactivity in the detector and can scatter in the LAr. The neutron's path ends when it gets captured on an atom, typically on some detector component. The neutron capture reaction typically releases a number of $\gamma$ rays, some of which can deposit energy in the LAr. The expected signal is thus a neutron scatter (high fprompt) followed by a $\gamma$ event (low fprompt).

The script works by first looking for a high fprompt $(0.6<$ fprompt $<0.8)$ event. Once it finds that event, it looks for a high qPE (qPE > 4000) low fprompt $(0.2<$ fprompt $<0.4$ ) event that occurs within $0.02 \mathrm{~s}$ before or after the high fprompt event. Once it finds this low fprompt event, it saves a selection of variables from both tagged events into a new ntuple. The script searches the entire window (both before and 


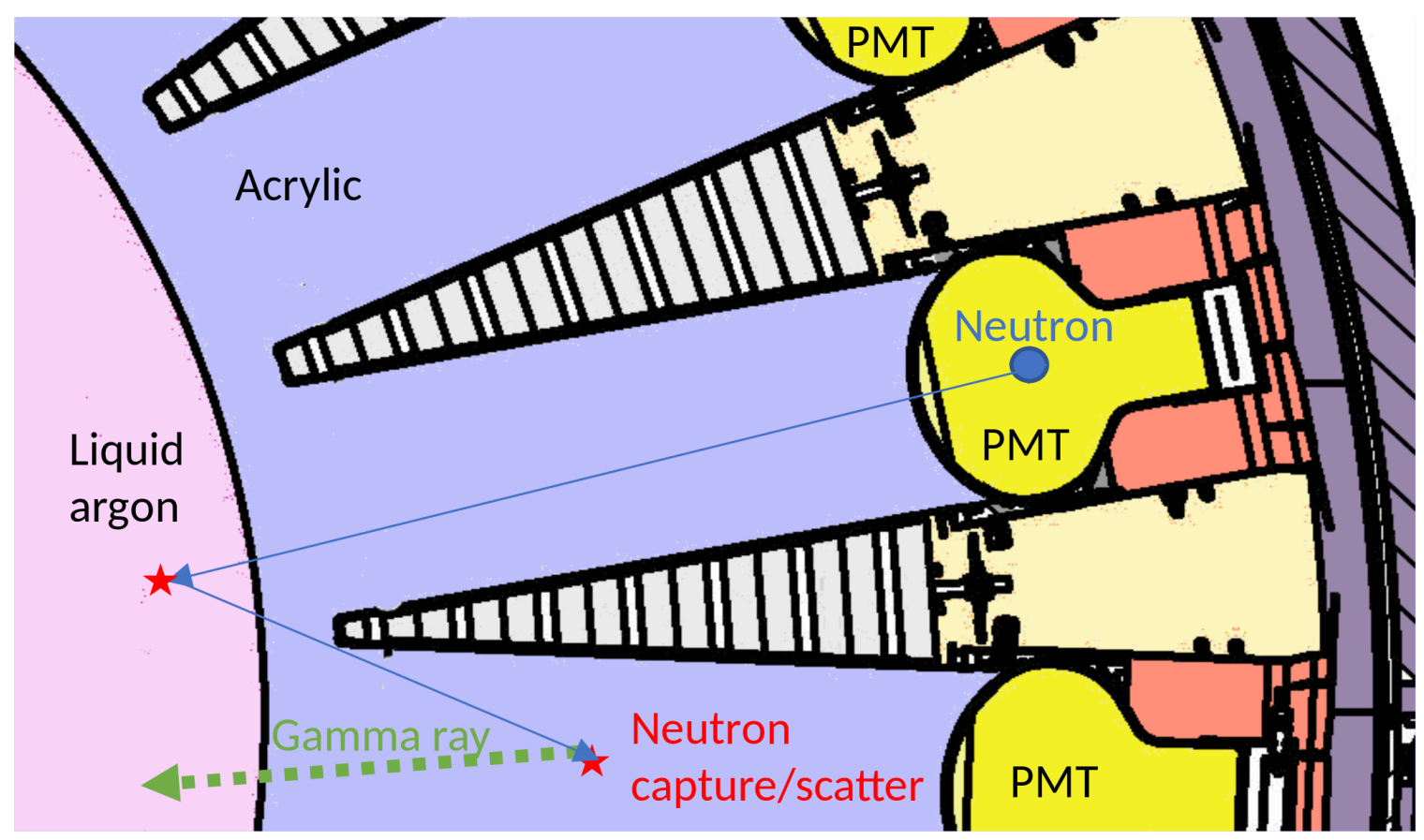

Figure 6.1: Illustration of a radiogenic neutron scatter in the LAr followed by a neutron capture. The neutron capture $\gamma$ then deposits energy in the LAr, allowing the neutron scatter event to be tagged as such.

after the high fprompt event) and adds an entry to the output ntuple for each low fprompt event found. It then moves on to the next high fprompt event and repeats the process for all events in the input file(s).

\subsection{Cuts}

In order to specifically select pairs of neutron recoils tagged with neutron capture $\gamma$ events, a set of cuts are applied. These cuts were developed as part of the previous analysis for the last WIMP search paper [1].

- Low Level: Removes:

- "Bad" triggers and externally triggered events

- Events that likely contain pileup, are effected by residual light in the argon, 
or are less than 20000 ns after the last trigger

- Events in which at least $40 \%$ of the total light collected is in a single PMT (primarily light guide Cerenkov events)

- Neck events

- Neutron Control Region:

$-100<\mathrm{qPE}<5000$ and $0.6<$ fprompt $<0.8$

- mblikelihoodR $<800$ ( $<787$ for MC) and mblikelihoodZ $<700$

- fmaxpe $<0.2$

- Capture $\gamma$ Region:

$-10000<$ qPE and $0.2<$ fprompt $<0.4$

- ROI:

- Additional neck cuts

- mblikelihoodR < 630 (608.5 for MC)

- ROI or strip

As shown above, the radial cut is different for MC vs data. This is because the position reconstruction fitter responds somewhat differently on data than it does on MC. This can be seen in Figure 6.2. To calculate the cut in MC that would be equivalent to a given cut in data, the acceptance of radial cuts ranging from $0 \mathrm{~mm}$ to $851 \mathrm{~mm}$ was calculated for ${ }^{39} \mathrm{Ar} \mathrm{MC}$ and data. The $\mathrm{MC}$ cut was then chosen to contain the same number of ${ }^{39} \mathrm{Ar}$ events as the data cut. The results for data cuts of 800 and 630 were $786.86 \pm 0.21$ and $608.55 \pm 0.39$ respectively. The data run used was 21399. The cuts used to select ${ }^{39} \mathrm{Ar}$ events in both $\mathrm{MC}$ and data were fprompt<0.4 \&\& fprompt $>0.2$ \&\& $\mathrm{qPE}>1000$ \&\& $\mathrm{qPE}<4000$ in addition to the usual low-level cuts. 


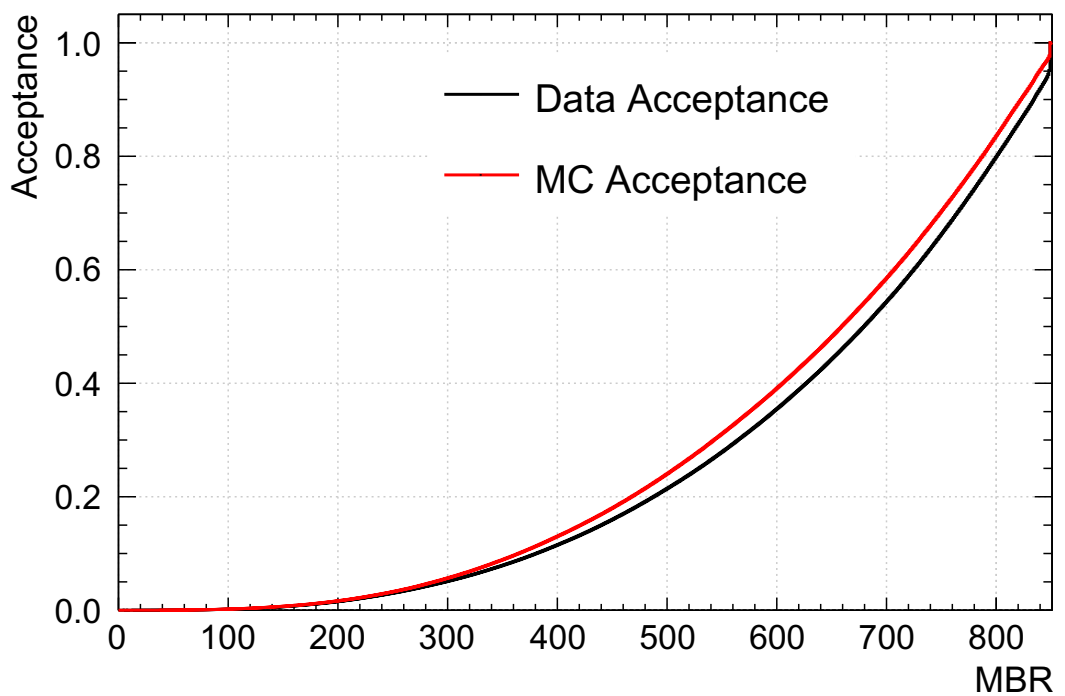

Figure 6.2: Acceptance vs MBR for ${ }^{39} \mathrm{Ar}$ data (run 21399) and MC. This was used to calculate MC MBR cuts for a given data cut by identifying the $\mathrm{MC}$ cut that would have the same acceptance as the data cut.

These cuts were applied on the output of the tagging script. The Low Level cuts were applied to both events in each tagged pair. The Neutron Control Region cuts were applied to the high fprompt events, and the Capture $\gamma$ cuts were applied to the low fprompt events.

\subsection{Time Window}

Not all the events selected by the above cuts will be pairs of neutron recoils and neutron capture $\gamma$ events. It is quite possible for a low fprompt event to occur within $20 \mathrm{~ms}$ of a high fprompt through random coincidence. To get an estimate of the number of random coincidence tags in our sample the time window was divided into 3 regions. Here $\Delta t=t_{n r}-t_{e r}$.

- $(-20 m s<\Delta t<0)$ : exclude from analysis. The nuclear recoil must happen before the neutron capture $\gamma$ is produced. 
- $(0<\Delta t<1 \mathrm{~ms})$ : Signal Window.

- $(1 m s<\Delta t<2 m s): 4 \%$ chance that neutron capture occurs here, so exclude from background estimation.

- $(2 m s<\Delta t<20 m s)$ : Random Window.

The distribution of low fprompt event time relative to the high fprompt event is shown in Figure 6.3. The signal window length was chosen based on a fit of the neutron capture time. This fit is shown in Figure 6.4. The capture time is determined by the neutron energy spectrum, the relative quantities of the materials that make up the detector, and the proximities of the materials to the LAr. In the case of DEAP3600 , this is thought to be dominated by captures on the LAr itself and on hydrogen in the acrylic. Based on the fit in Figure 6.4, the $1 \mathrm{~ms}$ window length will include $96.47 \pm 0.38 \%$ of neutron capture $\gamma$ events given an observed scatter of the neutron on liquid argon. At this point, the random coincidence backgrounds are increasing much faster than the efficiency. It was necessary to keep any contamination of the background window by neutron events negligible without including too many random coincidence events. Extending the window would make the signal to background ratio worse, while shrinking it would increase the probability of neutron events leaking into the background window, thereby inflating the background estimate and ultimately biasing the neutron estimate downwards.

The negative window contains a high rate of retriggers correlated with high energy low fprompt events. Surface ${ }^{214}$ Po $\alpha$ decays could also be present here, since the parent is a ${ }^{214} \mathrm{Bi} \beta$ decay and the ${ }^{214} \mathrm{Po}$ lifetime is $237 \mu \mathrm{s}$. It was therefore excluded from the analysis.

The tags in the Random Window provide an estimate of the background for the tags in the Signal Window. 


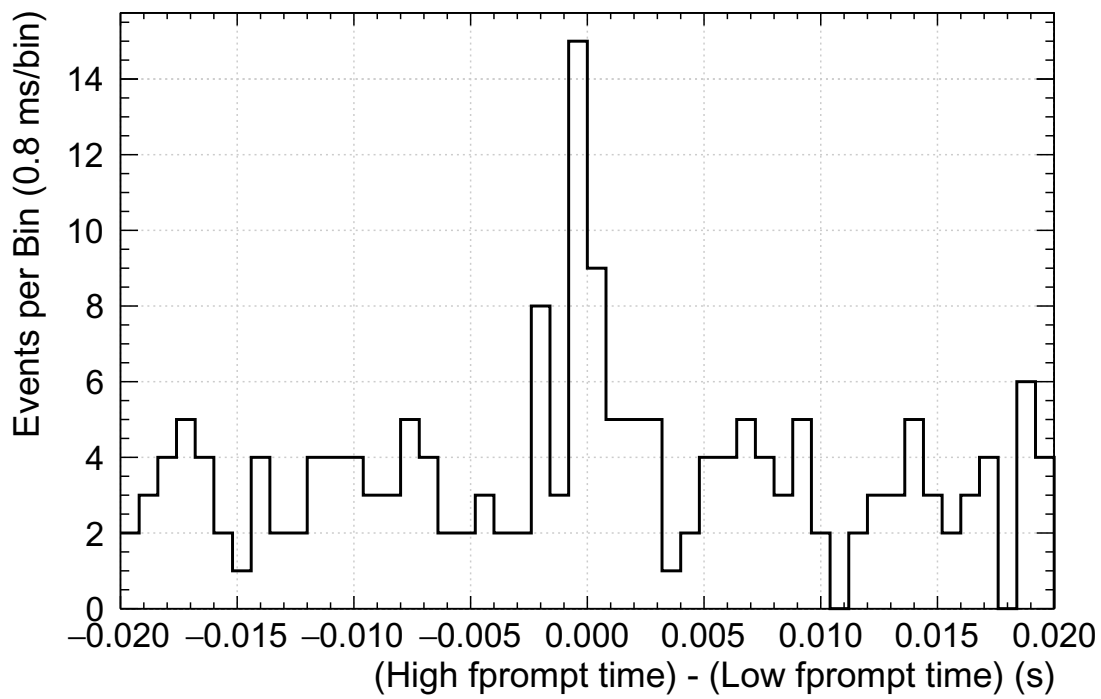

Figure 6.3: The distribution of the time between tagged pairs in the open physics dataset, including the negative window, where the low fprompt event occurs before the high fprompt event.

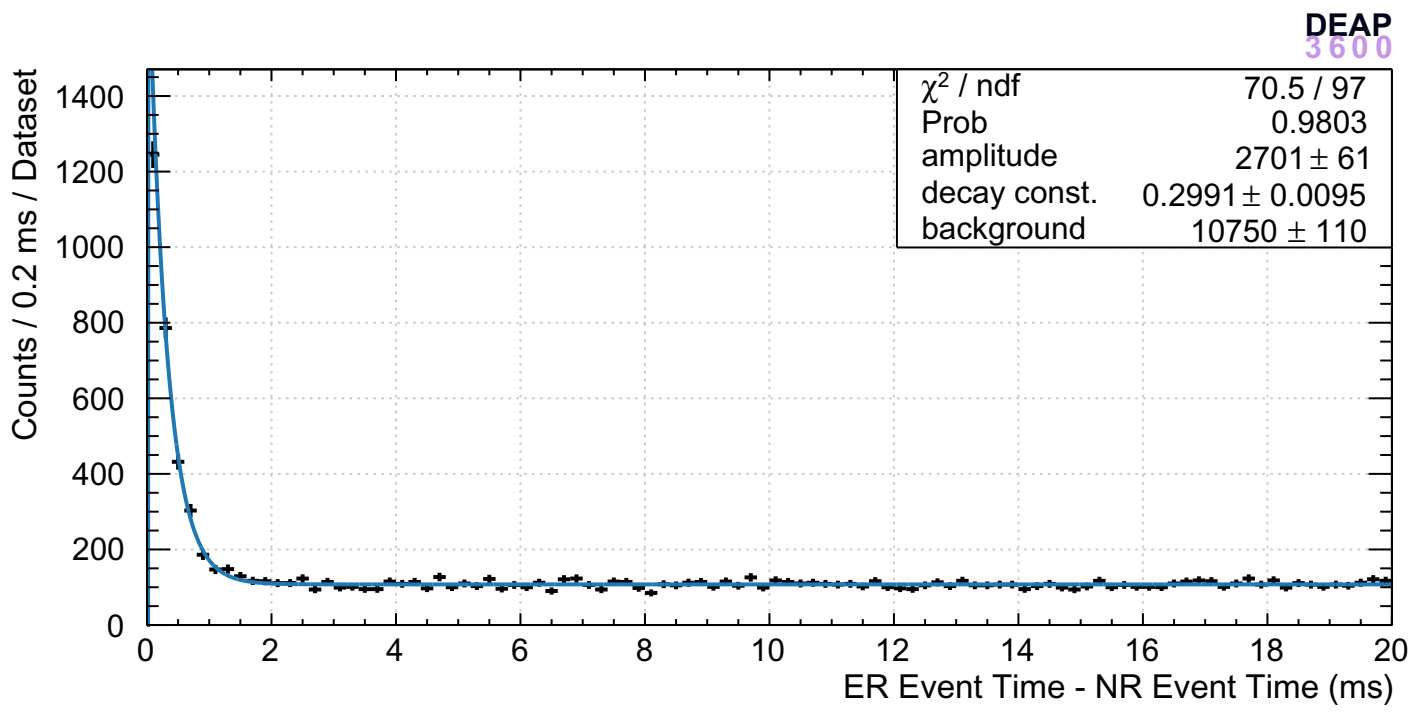

Figure 6.4: Fit of the time between high fprompt (neutron scatter candidate) and low fprompt (neutron capture $\gamma$ candidate) tags for the 2016DecAmBeSource AmBe runlist. The fit function is an exponential with a constant background. 


\subsection{Tagging Efficiency}

In order to get an estimate on the total number of neutron interactions that would survive our Neutron Control Region cuts, we need to know how often we expect to observe a capture $\gamma$ after seeing a neutron induced nuclear recoil. We will refer to this as the tagging efficiency. To get this number, a tagging analysis similar to the above was performed on AmBe data. The efficiency was obtained by subtracting the scaled Random Window qPE spectrum for the high fprompt tags from the corresponding Signal Window spectrum and dividing the result by the total number of high fprompt events in the data prior to tagging. This was repeated for a total of 6 runlists, distributed about 6 months apart. More could have been used, but each AmBe runlist requires a significant amount of CPU hours and disk space to process. Since the result had a small enough uncertainty, it was decided to not increase the number of AmBe runs used. The names of the runlists are:

- 2016DecAmBeSource

- AmBe_August2017_L0

- $\mathrm{AmBe}_{-}$December2017_L0

- AmBe_June2018_L0

- AmBe_January2019_L0

- AmBe_June2019_L0

The tagging efficiency for each run, along with a fit to determine the overall efficiency, is shown in Figure 6.5. From the fit, the overall tagging efficiency was determined to be $(21.65 \pm 0.25) \%$. 


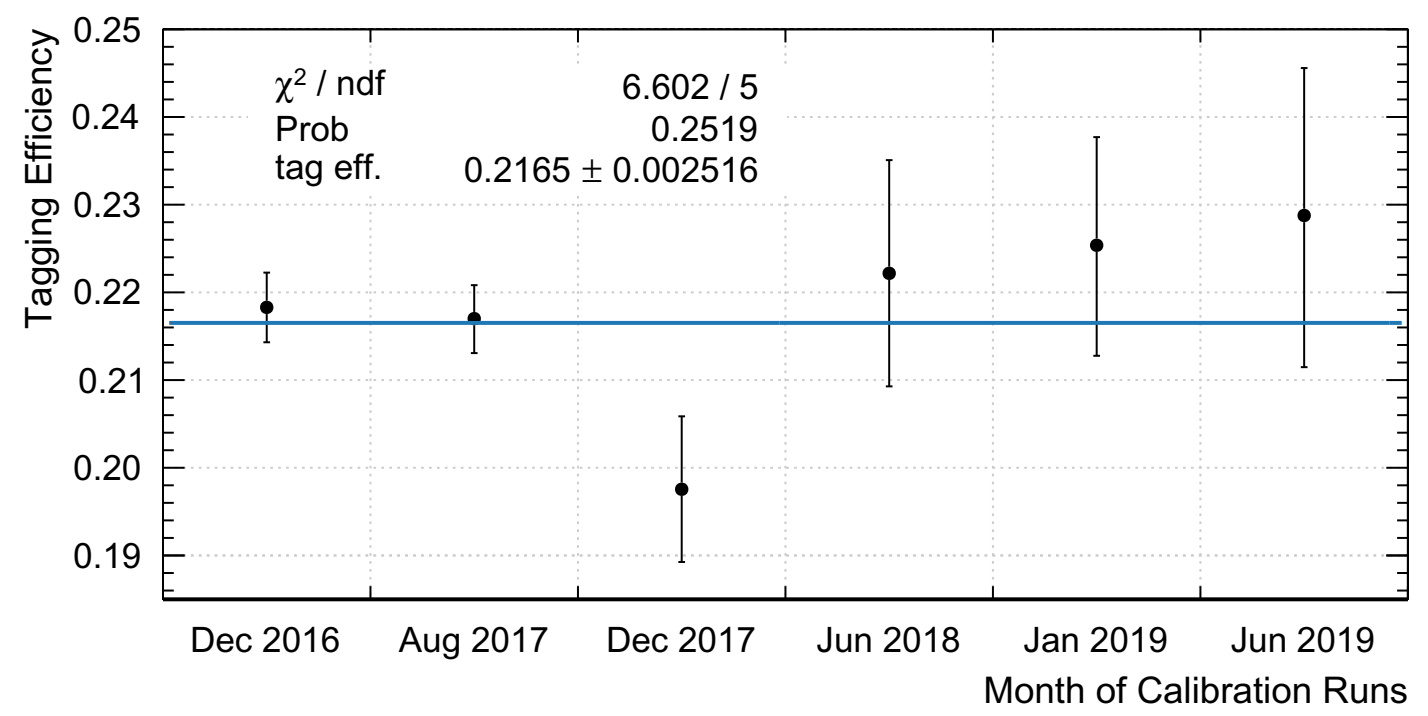

Figure 6.5: Tagging efficiency across several runlists. The runlists are: 1. 2016DecAmBeSource. 2. AmBe_August2017_L0. 3. AmBe_December2017_L0. 4. AmBe_June2018_L0. 5. AmBe_January2019_L0. 6. AmBe_June2019_L0. A horizontal line was fit to the tagging efficiency to get the overal efficiency used in this analysis. 


\subsection{Monte Carlo}

For the purpose of using the Monte Carlo to estimate the expected number of neutron scatter events in the ROI, a Control Region (CR) was defined. The cuts that define this CR were optimized to accept a high number of neutron scatter events (which can be tagged with neutron capture $\gamma \mathrm{s}$ ) without allowing for a large background of other event types (which would increase the number of random coincidence tags). This CR was defined by applying the Neutron Control Region cuts together with the Low Level cuts.

We can now estimate the total number of neutrons that would survive our Neutron Control Region cuts. What remains is to translate that into an estimate on the number of neutrons we expect in the WIMP ROI, at 100 to 240 qPE (also shown in Figure 6.7). This is done with the help of the MC. 100 million radiogenic neutrons were generated, and the leakages of events into the $\mathrm{CR}$ and in the ROI were obtained. Leakage is defined as $\frac{\text { Num. Events in CR or ROI }}{\text { Num. MC events generated }}$. Taking the ratio of these numbers gives the number of neutron triggers in the ROI for each neutron trigger in the CR.

This calculation was complicated by the fact that getting adequate neutron MC statistics is very computationally expensive. At the time of the previous analysis, ${ }^{238} \mathrm{U}$ PMT glass neutrons had the most statistics. The analysis described above was done for other types of radiogenic neutron MC, and the final results were all within each other's uncertainty. See Figure 6.6 for a comparison of the neutron energy spectra for the dominant neutron sources.

A collaborator estimated the systematic uncertainty in the MC model by running the $\mathrm{MC}$ with various parameters set to values allowed by their uncertainty, including PMT efficiency, PMT afterpulsing, the LAr refractive index, and the TPB scattering length. The final number for the ratio of neutrons in the ROI to neutrons in the

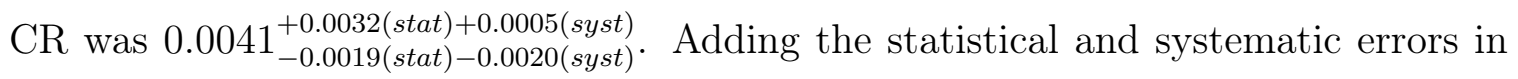




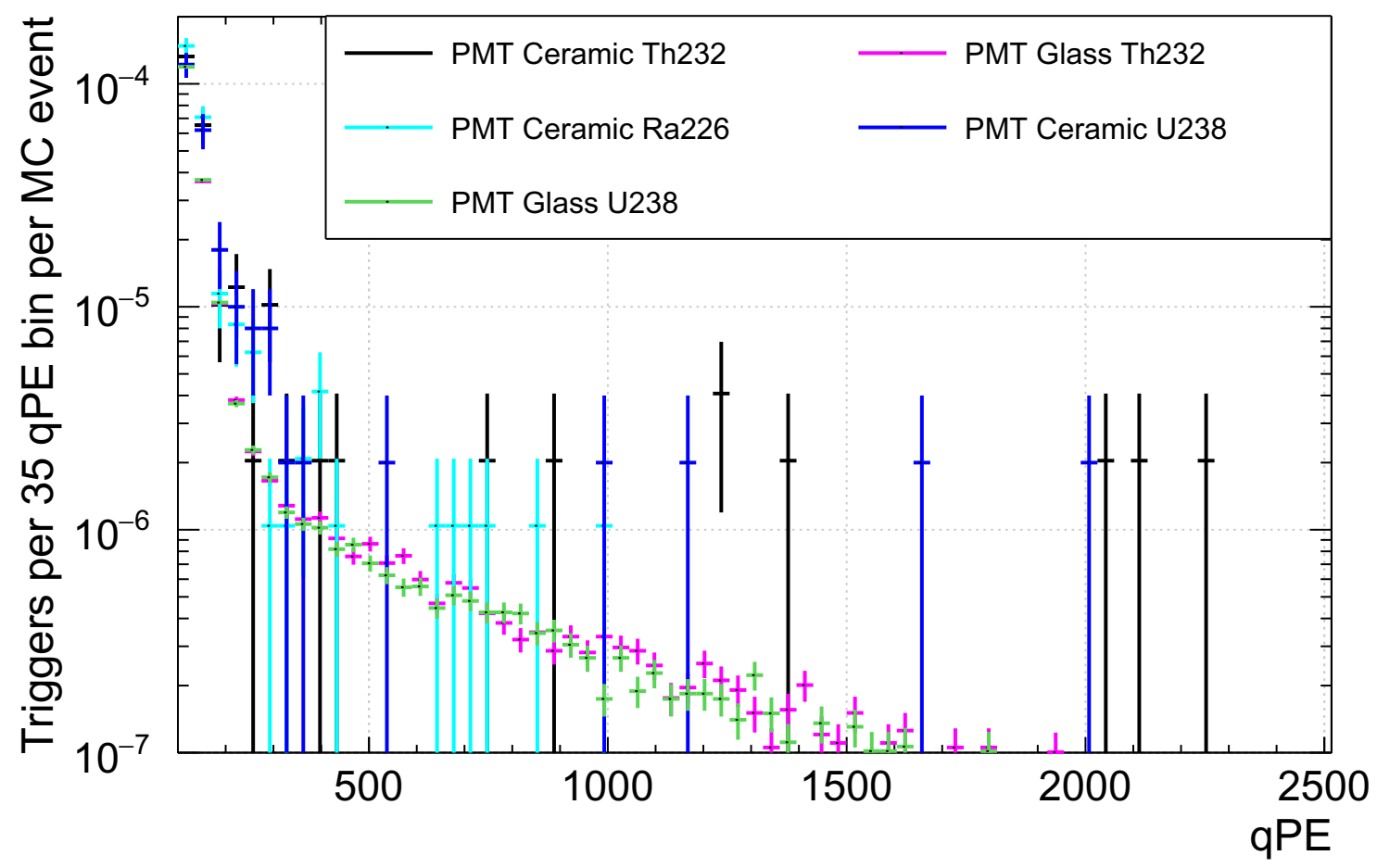

Figure 6.6: MC neutron recoils in LAr for some of the dominant radiogenic neutron sources. There were no events above 2500 qPE. The applied cuts were: $0.6<$ fprompt $<0.8$ and $100<\mathrm{qPE}<5000$. 
quadrature gives $0.0041_{-0.0028}^{+0.0032}$.

A large number of the $\mathrm{MC}$ events are neutron capture/scatter $\gamma$ interactions. Most of these are removed by the fprompt cut when the CR cuts are applied, but some $\gamma$ events still survive and, therefore, exist in the CR. In the data, these $\gamma$ events will not be tagged with the preceding neutron scatter, as they will be outside the $\gamma$ tagging region. These $\gamma$ events, therefore, have no effect in the data (except by possibly contributing occasionally to the random tagging background). However, they could have a significant effect on the calculation of the ratio of neutron scatters in the CR to neutron scatters in the ROI, since it is very unlikely that they follow the same distribution as neutron scatters in fprompt vs qPE space. The possible cases can be summarized as:

Case 1: (CR contribution; No ROI contribution)

- Neutron scatters and produces $\gamma$ before entering LAr

- $\gamma$ produces Cerenkov

- Neutron then captures

Case 2: (No CR contribution; No ROI contribution)

- Neutron captures before entering LAr

Case 3: (CR contribution; ROI contribution)

- Neutron scatters and produces $\gamma$ with Cerenkov

- Neutron scatters in LAr

- Neutron captures

Case 4: (CR contribution; ROI contribution)

- Neutron scatters in LAr 
- Neutron scatters and produces Cerenkov in acrylic

- Neutron captures

Case 5: (CR contribution; ROI contribution)

- Neutron scatters in LAr

- Neutron captures

The neutron MC file was processed to ensure that the events properly modelled the $\mathrm{CR}$ tags and types of ROI events that we are trying to estimate. Events that fell under cases 1, 3, 4, and 5 were entered into one branch (named "data_mc_t"), and events that fell under cases 3, 4, and 5 were entered into another branch (named "data_mc"). In addition, to be considered as a potential CR event and entered into the "data_mc_t" branch, it was required that a neutron track exist such that its last step occurred after the end of the event waveform, and less than $1 \mathrm{~ms}$ after the event triggered. This condition ensured that the event represented one which could be tagged. The primary purpose of this is to exclude Cerenkov and other low energy high fprompt $\gamma$ events from the MC CR which would not be followed by a correlated high energy low fprompt event resulting from a neutron capture or inellastic scatter. These events would not be tagged in real data (except through random coincidence, which is already accounted for), so including them in the MC CR artificially lowers the neutron expectation. To be considered a potential ROI event, a neutron track must also lose some energy in the LAr during the time covered by the waveform of the triggered event.

Unfortunately, the RAW branch info needed for this was pruned when the initial $\mathrm{MC}$ was run. It was, therefore, necessary to reprocess the MC events that produced triggers and also contained a neutron track that entered the LAr. However, the variable values differed slightly from the initial $\mathrm{MC}$ dataset due to the fact that the 
DAQ processor uses a random number generator to simulate the noise in the event waveforms. In order to preserve the original sequence of random numbers, it would have been necessary to regenerate the entire MC dataset. The overall effect of this was that the MC event variables that were ultimately used in this study differed slightly from their original values.

Of the events in the resulting MC input file, 2740 of them passed the CR cuts, and 19 passed the ROI cuts. The previous analysis (used in [1]) found an upper systematic error of $10 \%$ and a lower systematic error of $50 \%$ on the neutron leakage into each

of the two regions. Reusing these values here, we get $\left(2.74_{-0.05(\text { stat })-1.37(\text { syst })}^{+0.05(\text { stat })+0.27(\text { st })} \times 10^{-5}\right.$ triggers in the CR per neutron generated and $\left(1.70_{-0.41(\text { stat })-0.85(\text { syst })}^{+0.52(\text { stat })+0.17(\text { st })}\right) \times 10^{-7}$ triggers in the ROI per neutron generated. Taking the ratio of these two numbers, we get $0.0062_{-0.0015(\text { stat })+0.0003(\text { syst })}^{+0.001 \text { (syst) }}$ neutrons in the ROI for every neutron in the CR. Adding the stat. and syst. errors in quadrature, we get $0.0062_{-0.0034}^{+0.0020}$. Note that this number is unchanged between the 247 day ROI and the 802 day ROI.

\subsection{Results}

Combining all this we can get the estimated number of neutrons in the ROI.

The above analysis was applied on the following runlists (length with deadtime correction in brackets):

- PhysicsTrigger_November2016ToDecember2017_L2 (272.558 days)

- PhysicsTrigger_OpenData2018_L2 (50.0630 days)

- PhysicsTrigger_OpenData2019_L2 (53.5558 days)

- PhysicsTrigger_OpenData2020_L2 (12.2223 days)

The result is summarized in table 6.1, along with the numbers from the previous analysis. 


\begin{tabular}{c|c|c}
\hline \hline & Last Paper & This Analysis \\
\hline Uncorrected Livetime & 246.71 days & 388.399 days \\
Tags in Signal Window & 7 & 10 \\
Tags in Random Window & 34 & 89 \\
Tagging Efficiency & $0.225 \pm 0.005$ & $0.2165 \pm 0.0025$ \\
Uncertainty Calculation & Feldman-Cousins & Rolke $|58|$ \\
Number of Neutrons in CR & $23.1_{-14.3}^{+16.9}$ & $23_{-13}^{+16}$ \\
Neutrons in ROI per CR & $0.0041_{-0.0028}^{+0.0032}$ & $0.0062_{-0.0034}^{+0.0021}$ \\
Number of Neutrons in ROI & $0.10_{-0.09}^{+0.10}$ & $0.15_{-0.12}^{+0.11}$ \\
\hline \hline
\end{tabular}

Table 6.1: Result of tagging analysis of neutron induced nuclear recoils with neutron capture $\gamma$ events. Uncertainties here include both statistical and systematic contributions.

As part of blinding we need to know the number of events in the three blinding strips and the 802 day ROI. The CR does not change, but we need the ratio of MC neutrons in each strip per neutron in the CR. This is summarized in table 6.2. The expected numbers of events for each region are in table 6.3. Note that these strips do not overlap. Strip 1, for example, only includes the region between the strip 1 and strip 2 boundaries, and does not include any of the area in strip 2. The strips are plotted in Figure 6.7.

\begin{tabular}{c|c}
\hline \hline Neutrons in Blinded Reg. per CR & $0.0310_{-0.0159}^{+0.0056}$ \\
Neutrons in Strip 1 per CR & $0.0033_{-0.0020}^{+0.0016}$ \\
Neutrons in Strip 2 per CR & $0.0139_{-0.0073}^{+0.0032}$ \\
Neutrons in Strip 3 per CR & $0.0139_{-0.0073}^{+0.0032}$ \\
Neutrons in 802 Day ROI per CR & $0.0062_{-0.0034}^{+0.0021}$ \\
\hline \hline
\end{tabular}

Table 6.2: Ratios of MC neutrons in blinding regions to MC neutrons in CR. Uncertainties here include both statistical and systematic contributions.

Figure 6.7 shows neutron tags in fprompt vs qPE. The one survivor is event 


\begin{tabular}{c|c|c}
\hline \hline Region & Neutron in Open Data & Neutrons in 802 Day Dataset \\
Blinded Reg. & $0.73_{-0.56}^{+0.52}$ & $1.5_{-1.1}^{+1.1}$ \\
Strip 1 & $0.077_{-0.064}^{+0.065}$ & $0.16_{-0.13}^{+0.13}$ \\
Strip 2 & $0.32_{-0.25}^{+0.24}$ & $0.67_{-0.52}^{+0.49}$ \\
Strip 3 & $0.32_{-0.25}^{+0.24}$ & $0.67_{-0.52}^{+0.49}$ \\
802 Day ROI & $0.15_{-0.12}^{+0.11}$ & $0.30_{-0.24}^{+0.23}$ \\
\hline \hline
\end{tabular}

Table 6.3: Expected numbers of radiogenic neutrons in blinding strips and ROI. Here the 802 day dataset is assumed to be 802 live days, so a scale factor of 2.06489 is applied to the numbers in the open data column to get the numbers in the 802 day dataset column. Uncertainties here include both statistical and systematic contributions.

250617926 from run 21162. The expected number of tagged neutrons in the blinded region of the open data is $0.73_{-0.56}^{+0.52} \times 0.2163 \pm 0.0025=0.16_{-0.12}^{+0.11}$. The probability of seeing at least 1 event in the blinded region of the open dataset is, therefore, $0.15_{-0.11}^{+0.09}$.

\subsubsection{Uncertainties}

The final result has a large uncertainty. In order to understand the drivers of this uncertainty, table 6.4 lists the contributions of the various sources of uncertainty to the final result. As can be seen, the uncertainty is primarily statistical, and is driven by the low number of counts in the data.

\subsection{Tagging Cut}

An easy way to remove some of the predicted number of neutrons from the dataset is to run the time coincidence tag and remove all tagged events in the signal window. The cut efficiency will be equal to the tagging efficiency of $0.2165 \pm 0.0025$. The 


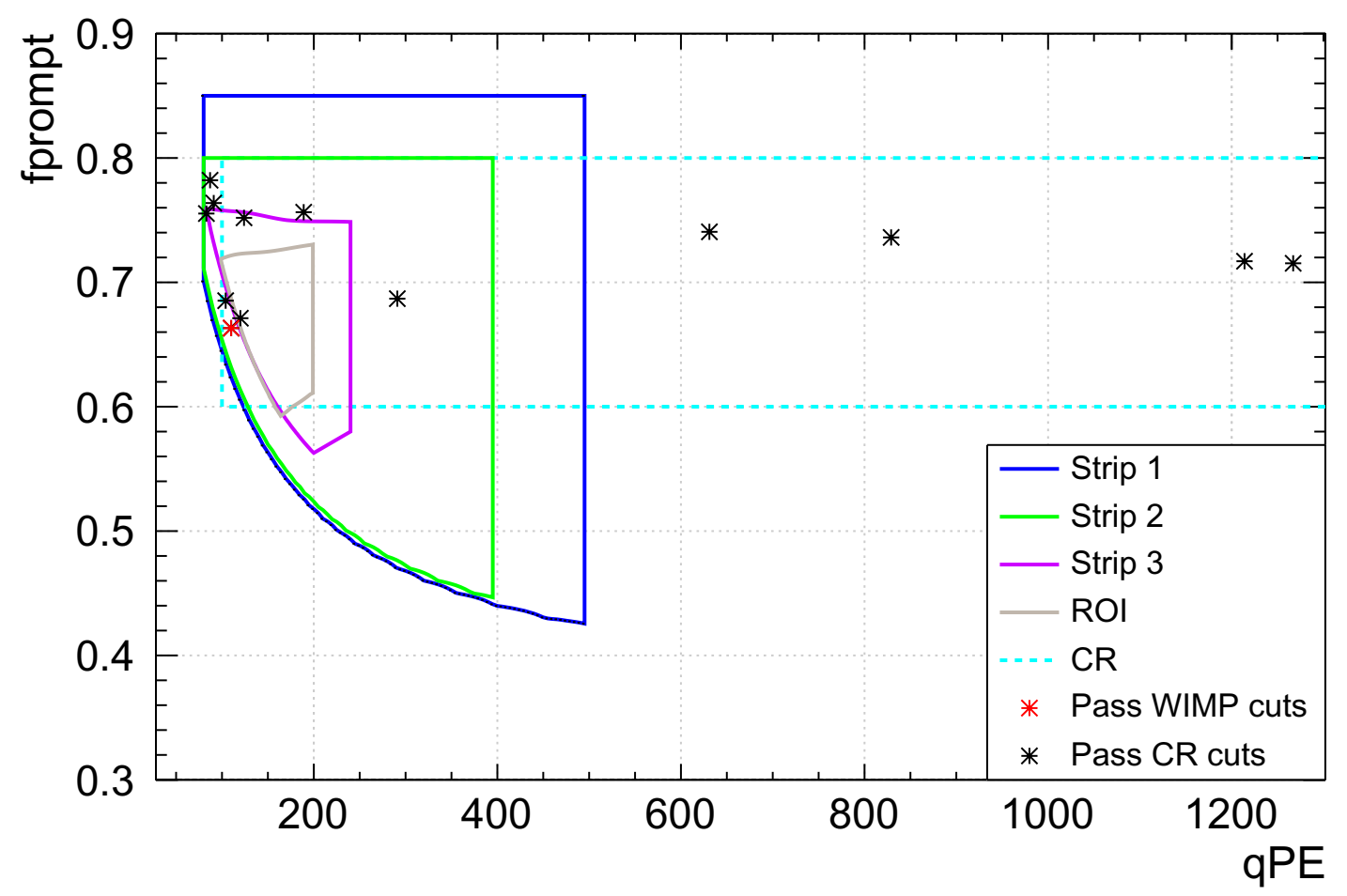

Figure 6.7: Tagged radiogenic neutron candidates. The black events are tags that pass all control region cuts (not including fprompt and qPE cuts), and the red events are those which survive the cuts used in [1]. Here the fprompt and qPE cuts have been replaced with: strip1 OR ( $\mathrm{PPE}>495$ AND fprompt $<0.85$ AND fprompt $>0.425595)$. The control region (CR), WIMP ROI, and the three blinding strips are also shown. 


\begin{tabular}{c|c}
\hline \hline & Uncertainty \\
\hline Total Uncertainty on Current Analysis (open dataset) & $(+0.11,-0.12)$ \\
Total Syst Error (MC Model) & $(+0.02,-0.07)$ \\
Total Stat Error & $(+0.11,-0.09)$ \\
MC Stat Error & $(+0.04,-0.03)$ \\
Data Stat Error & $(+0.10,-0.08)$ \\
Tagging Efficiency Error & $(+0.01,-0.02)$ \\
\hline \hline
\end{tabular}

Table 6.4: Breakdown of uncertainty on final result for ROI.

acceptance loss is equal to:

$$
\frac{N_{\text {tags }_{\text {ran }}} \times L_{\text {sig }} / L_{\text {ran }}}{N_{\text {events }}}=\frac{2,660,002 / 19}{63,782,850}=0.002,
$$

where $N_{\text {tags }}$ is the total number of tags in the random window, $L_{\text {sig }}$ and $L_{\text {ran }}$ are the lengths of the signal and random time windows, and $N_{\text {events }}$ is the total number of high fprompt events that the tagging analysis code looked at. The loss in acceptance due to this cut is therefore $0.2 \%$. The fraction of neutrons that will survive this cut is $0.7837 \pm 0.0025$.

The resulting neutron background expectations after applying this cut are shown in table 6.5 .

\subsection{Conclusion}

The total number of neutrons expected in the open data is $0.15_{-0.12}^{+0.11}$. Scaling by the ratio of total data to open data (1.80063), we get an expectation of $0.30_{-0.24}^{+0.23}$ for the full 3 year dataset. 


\begin{tabular}{c|c|c}
\hline \hline Region & Neutron in Open Data & Neutrons in 802 Day Dataset \\
Blinded Reg. & $0.57_{-0.44}^{+0.41}$ & $1.17_{-0.90}^{+0.85}$ \\
Strip 1 & $0.060_{-0.050}^{+0.0051}$ & $0.12_{-0.10}^{+0.11}$ \\
Strip 2 & $0.25_{-0.20}^{+0.19}$ & $0.52_{-0.41}^{+0.39}$ \\
Strip 3 & $0.25_{-0.20}^{+0.19}$ & $0.52_{-0.41}^{+0.39}$ \\
802 Day ROI & $0.114_{-0.091}^{+0.088}$ & $0.23_{-0.19}^{+0.18}$ \\
\hline \hline
\end{tabular}

Table 6.5: Expected numbers of radiogenic neutrons in blinding strips and ROI after applying neutron tag cut. Uncertainties here include both statistical and systematic contributions. 


\section{Chapter 7}

\section{Search for 5.5 MeV Solar Axions}

As discussed in Chapter 2, several proposed extensions to the standard model predict the existence of axions or axion-like-particles (ALPs). If axions exist, it is possible that they are being produced in the sun. One possible production mechanism is the production of a $5.5 \mathrm{MeV}$ axion in place of a $5.5 \mathrm{MeV}$ photon in the proton-deuterium fusion reaction. DEAP has sensitivity to searching for evidence of these axions interacting in the LAr, depending on the axion mass and coupling constant to other particles. These interactions would be two orders of magnitude more energetic than WIMP interactions.

\subsection{Expected Signal and Background}

Interactions of $5.5 \mathrm{MeV}$ solar axions with LAr atoms would manifest as a peak in the electron recoil band at this energy. The dominant backgrounds here are $\gamma$ events arising from the intrinsic radioactivity of the detector.

\subsubsection{Signal}

The signal was modelled by running Monte Carlo simulations of the four relevant axion interactions within the detector model. In the cases where multiple primary particles 


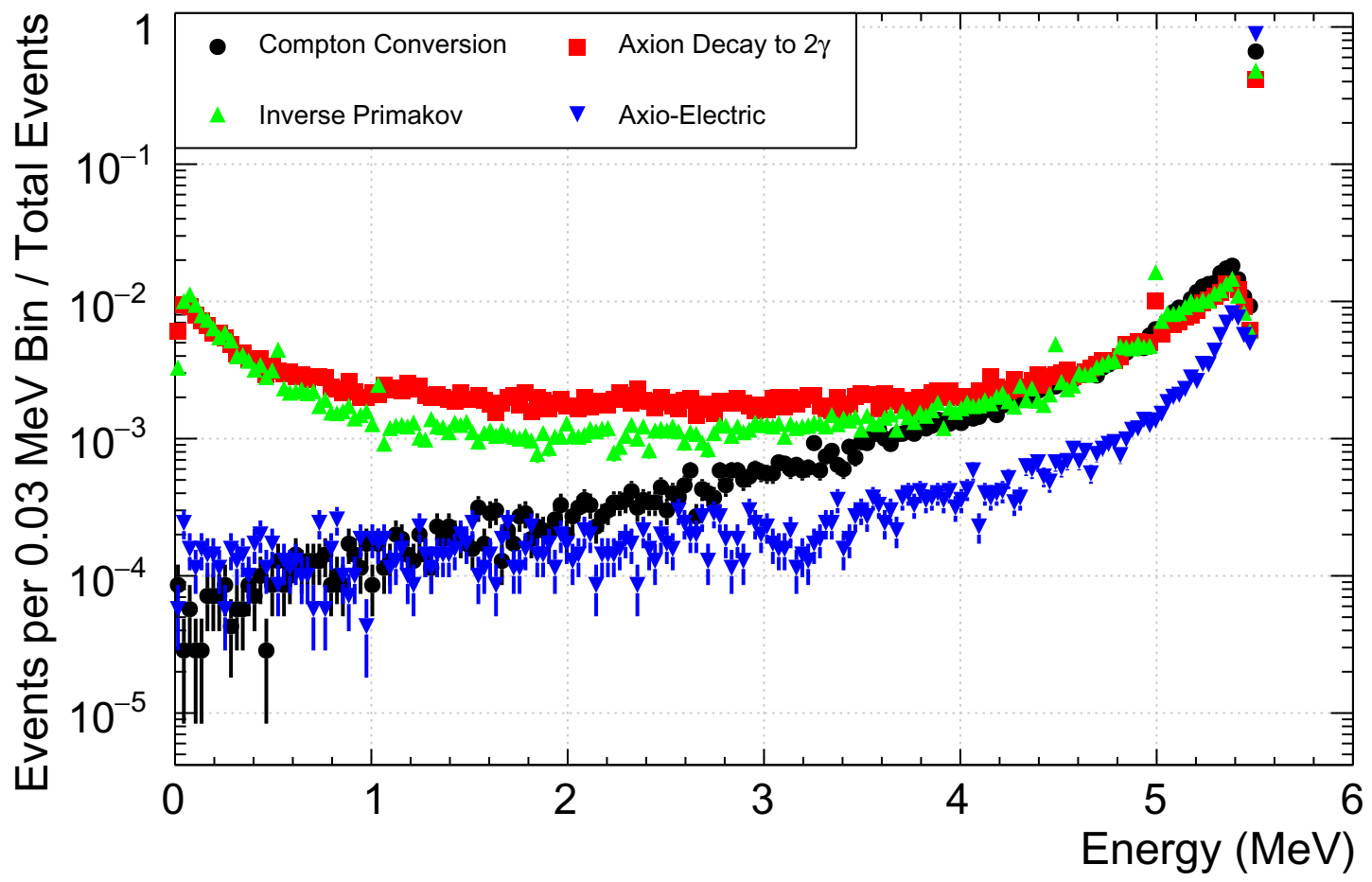

Figure 7.1: Total energy deposited in the detector after an axion interaction from each of the four interactions being searched for. The axion mass is set to $49 \mathrm{keV}$ for the Compton conversion and axion decay processes.

are produced (Compton conversion and axion decay), the Monte Carlo interaction vertex generators were implemented as an external python script that computed the momentum of the primary particles produced in the interaction, randomly distributed according to the corresponding differential cross section. This script fed the results of these calculations into RAT's external event generator, which in turn simulated the interactions of the primary particles and their secondaries within the detector. The output of these simulations is shown in Figure 7.1. These interactions are also mass dependant. In the relativistic axion case, there is no difference in the energy spectrum. However, as the mass approaches $5.5 \mathrm{MeV}$, the energy spectra start to differ significantly from one another. This is illustrated in Figure 7.2 . 

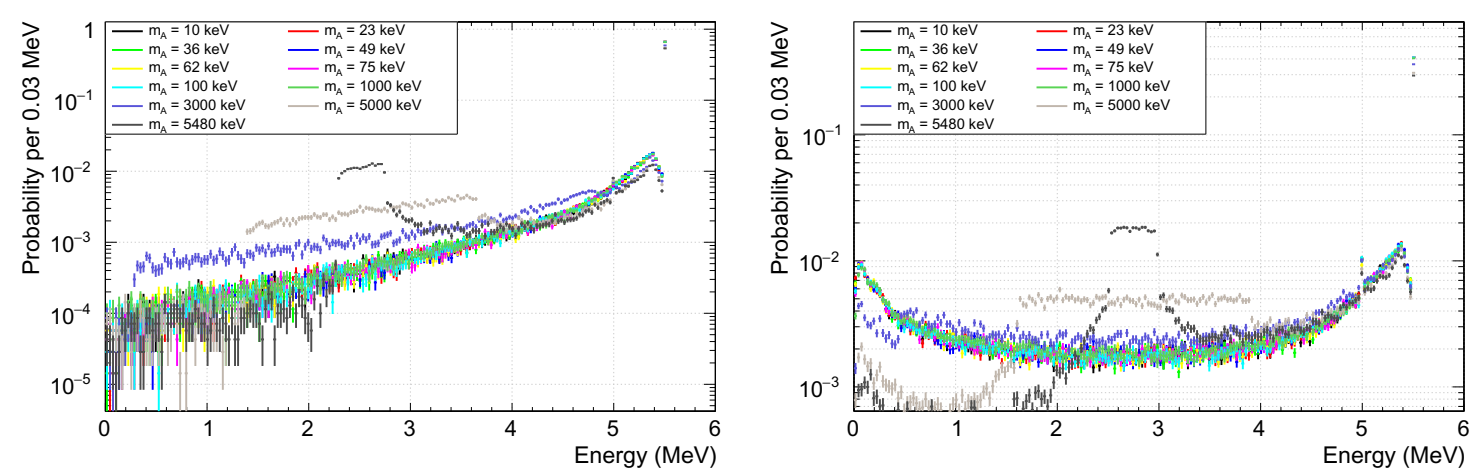

Figure 7.2: Total energy deposited in the detector after a Compton conversion (left) and an axion decay to two $\gamma_{\mathrm{s}}$ (right), both of which produce two primary particles. Each plot shows the spectrum produced by a given axion mass. As can be seen, for both interactions there is no noticeable change among the spectra until the axion mass reaches $3 \mathrm{MeV}$, where the axion starts to become non-relativistic.

\subsubsection{Backgrounds in Model}

\section{Neutron Capture $\gamma \mathbf{s}$}

The DEAP Collaboration has previously published the results of a fit to most of the electron-recoil spectrum in DEAP-3600 [59]. The fit is shown in Figure 7.3. This work (by a collaborator) showed that the only significant background around $5 \mathrm{MeV}$ is expected to be from neutrons produced as a result of radioactive decay (such as $(\alpha, n)$ reactions) within the detector components. These background events are not the result of direct interactions of neutrons with the LAr, but are the result of interactions of neutron capture $\gamma \mathrm{s}$ which are produced when a neutron captures on a detector component, or, in some cases, on an argon atom in the LAr target. This process is illustrated in Figure 7.4 .

${ }^{208} \mathrm{Tl}$

As can be seen from Figure 7.3, non-neutron-induced backgrounds dominate below $4 \mathrm{MeV}$. These are from $\beta$ and $\gamma$ particles produced in radioactive decays. However, 


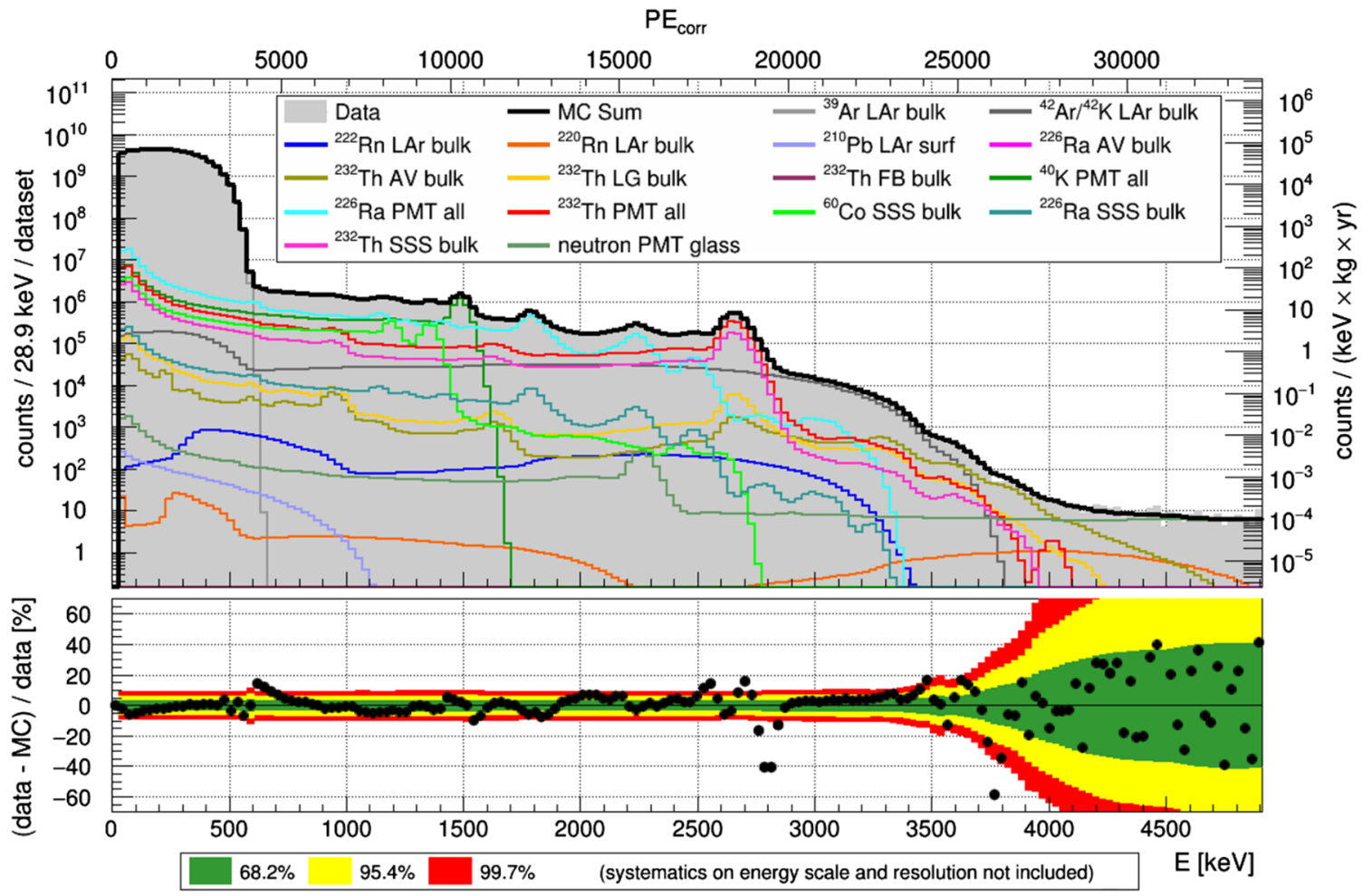

Figure 7.3: Fit of the DEAP-3600 electron-recoil spectrum (by a collaborator), taken from $|59|$.

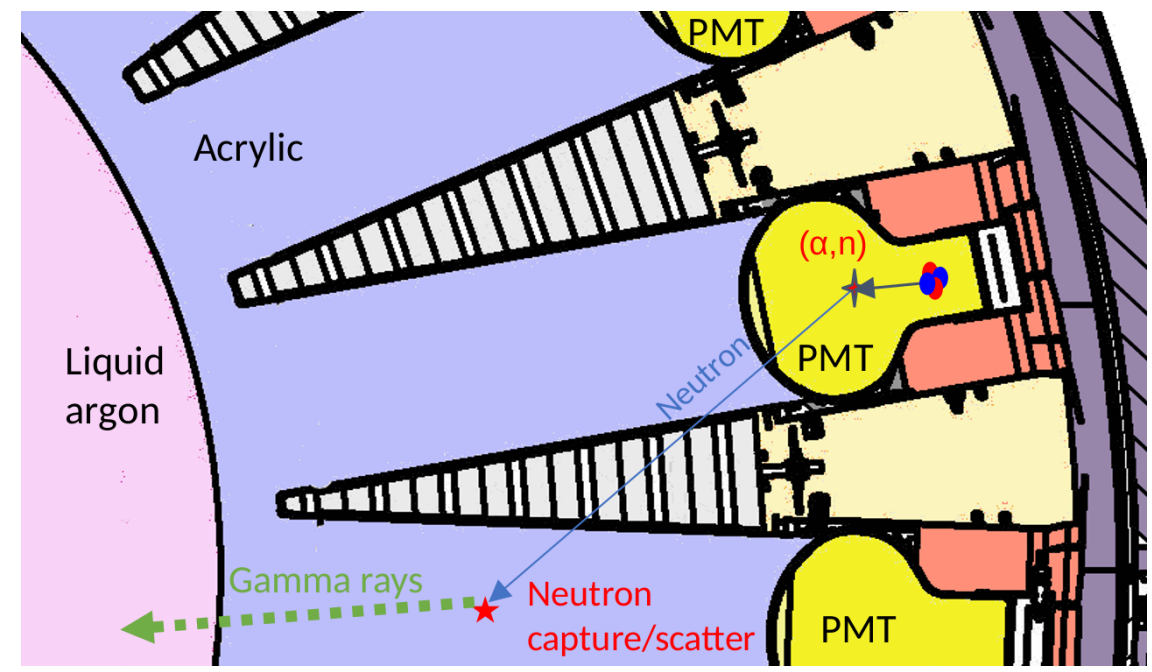

Figure 7.4: Illustration of the production mechanism of the neutron capture $\gamma$ background, which dominates the region near $5.5 \mathrm{MeV}$. 


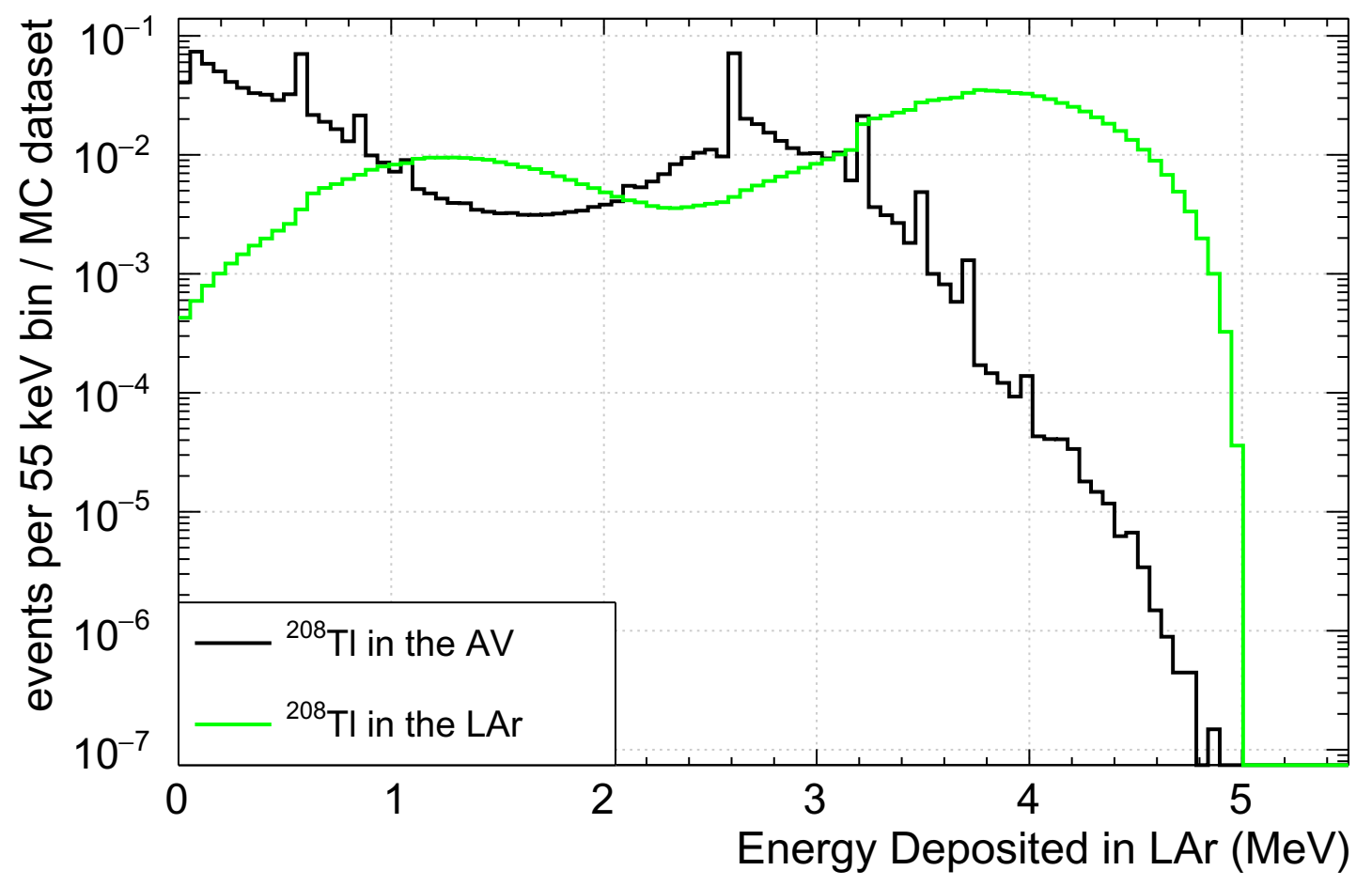

Figure 7.5: MC-generated ${ }^{208} \mathrm{Tl}$ spectra for decays generated in the AV acrylic bulk (black) and in the LAr bulk (green).

the fits performed in the axion search started at $30000 \mathrm{PE}(4.35 \mathrm{MeV})$, well above the endpoints of most of these backgrounds. The one exception is from decays of ${ }^{208} \mathrm{Tl}$, which is part of the "232 Th AV bulk" and " ${ }^{220}$ Rn LAr bulk" components in Figure 7.3 . MC-generated spectra of these decays are shown in Figure 7.5 .

\subsection{Overview of Procedure}

The main steps in this project are, roughly:

- Generate the MC datasets for calibration data, axion signal, and background events

- Process the energy depositions in the MC into simulated detector triggers 
- Fit the MC calibration model to the AmBe calibration dataset to extract the detector energy response function parameters

- Using the constraints from the calibration fit, run a second (blinded) fit of the background models to the 802 day physics dataset

- Finally, run a third fit (unblinded) of the axion signal and backgrounds model to the same 802 day dataset and extract the final result

\subsubsection{Blinding and Unblinding Procedure}

This is a blind analysis, so a portion of the physics data was excluded from the fit and from any plots that were made or viewed by the author. This was set to be all physics (i.e., non-calibration) data with $0.2<$ fprompt $<0.4$ and 36,000 $<$ qPE $<$ 39,000 , which corresponds to $5.26<\mathrm{MeV}<5.71$ in the electron-recoil band. The blinding is applied after the light yield correction has been performed. The blinded region is hard-coded into the fitting software, and prevents the data in the blinded region from being filled into the data histogram. Once approval has been obtained from the collaboration, the blinding will be turned off and the fitter will have access to the entire dataset. The analysis will be frozen before the data is unblinded and the resulting exclusion limit or excess will be published.

\subsection{Generating Monte Carlo Datasets}

The following Monte Carlo datasets were produced:

- AmBe calibration source neutrons and $\gamma_{\mathrm{s}}$

- $(\alpha, n)$ neutrons produced from ${ }^{238} \mathrm{U}$ decays in the PMT glass

- ${ }^{208} \mathrm{Tl}$ decays in the $\mathrm{AV}$ 
- ${ }^{208} \mathrm{Tl}$ decays in the LAr

- Axion Compton conversion for various axion masses

- Axion decay to $2 \gamma$ for various axion masses

- Inverse Primakov conversion

- Axio-electric effect

The software infrastructure was already in place for the simulation of PMT glass neutrons, ${ }^{208} \mathrm{Tl}$ decays, Inverse Primakov conversion, and the Axio-electric effect. The other datasets required the creation of additional Monte Carlo generators and the implementation of the AmBe source geometry.

\subsubsection{AmBe MC Generator and Geometry}

An AmBe source event generator was created by colleagues for previous projects. The correlations between the neutron energy and the number of $\gamma$ rays emitted from the source were not fully implemented, however. The implementation of these correlations was finished by the author in preparation for this work. The Doppler shift in the $\gamma$ ray energy was also added, taking into account the motion of the ${ }^{12} \mathrm{C}$ isotope within the AmBe source. This motion was determined by the initial momentum of the incident $\alpha$ particle, the change in momentum due to the emission of the neutron, and the number of $\gamma$ rays that were produced in the de-excitation of the ${ }^{12} \mathrm{C}$ nucleus.

The AmBe source had been deployed into one of the vertical calibration tubes (referred to as "Cal" tubes) that run along the outside of the steel shell (see Figure 3.9). While the calibration tube geometry was already implemented within the Monte Carlo package, the AmBe source and deployment apparatus were not. Since many of the materials that make up these objects contain isotopes with non-negligible neutron capture cross-sections, these objects and their materials were added to the simulation. 


\section{AmBe Source Geometry}

The AmBe source is contained within 2 encapsulations. The first encapsulation was performed by the manufacturer. This contains the radioactive powder that generates the neutrons. All measurements of the source activity were done after this encapsulation, so, for the purposes of simulation, the source in its original encapsulation is treated as a black box and modeled as a vacuum. The second encapsulation was performed by other members of the DEAP Collaboration at SNOLAB. This second encapsulation can be broken down into an inner component and an outer component. The inner component is a copper foil, held in place with a layer of solder (see Figure 7.6, left). The outer component is made of one steel cylinder inserted into another (see Figure 7.6 , right). The air within this cylinder was evacuated, and the cylinder was sealed with a copper gasket using a knife edge on the smaller cylinder. In the implementation of the Monte Carlo geometry for this project, the two cylinders are simulated as a single volume, and the copper gasket is within this volume. The cylinder was also covered in epoxy resin after being fastened together. The Monte Carlo implementation is shown in Figure 7.7 .

Important for the purpose of the neutron capture simulation is the fact that the doubly-encapsulated source was placed between two NaI scintillating crystal units (see Figure 7.8 , bottom). The NaI crystals produces scintillation light when particles emitted by the AmBe source pass through them. This light is detected by the tagging PMT attached to the end of each crystal. The tagging PMTs were not used for this work, but the geometry is relevant for the extra material near the source that could capture neutrons and change the flux of the particles entering the LAr. The body of the NaI unit is aluminum, and it is covered in the center by a MuMetal magnetic shield, which surrounds the PMT within the unit. Surrounding the well containing the source, and above it, is the NaI crystal. Around the walls and bottom of the 

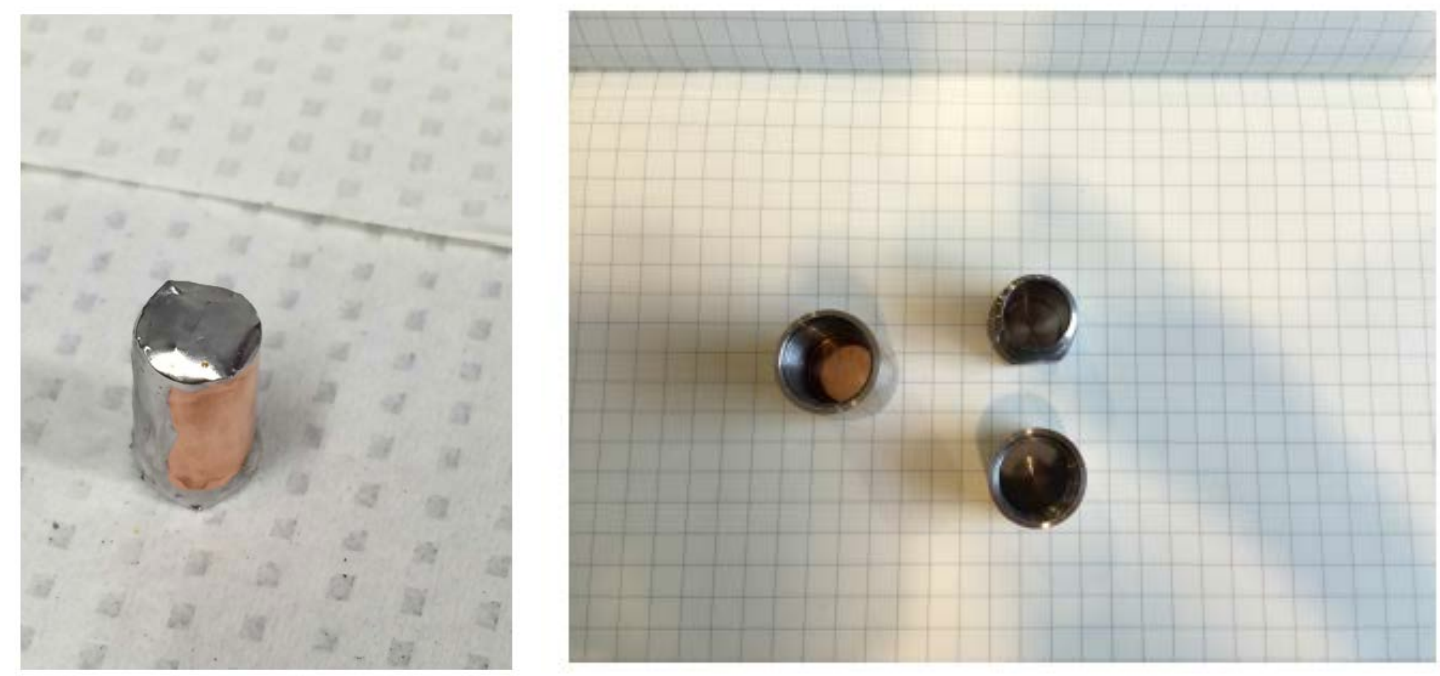

Figure 7.6: Left: The AmBe source after performing the inner (copper foil) portion of the DEAP encapsulation. Right: The AmBe source next to the outer encapsulation components (the two metal cylinders).

crystal is a reflector, modeled here as tyvek. Above the crystal is the PMT, modeled as a vacuum-filled $1 \mathrm{~mm}$ thick glass cylinder. The NaI unit is read out by wires from the end opposite the well. The wires and PMT readout electronics were not simulated. All these materials were implemented within the simulation, as well as various metal and plastic components not mentioned here. The source deployment geometry is the only place within the detector where Iodine is present in more than trace quantities. The full simulated deployment geometry can be seen in Figure 7.8 .

\subsubsection{Axion MC Generators}

The four axion processes under study (Figure 7.9) were simulated within the DEAP Monte Carlo package. The Inverse Primakov conversion process simply produces a $5.5 \mathrm{MeV} \gamma$ ray, which can be easily simulated within the already existing software framework. The same applies for the axio-electric effect, which would produce an electron with 5.5 MeV of kinetic energy. The other two processes required the creation of new Monte Carlo generators. 


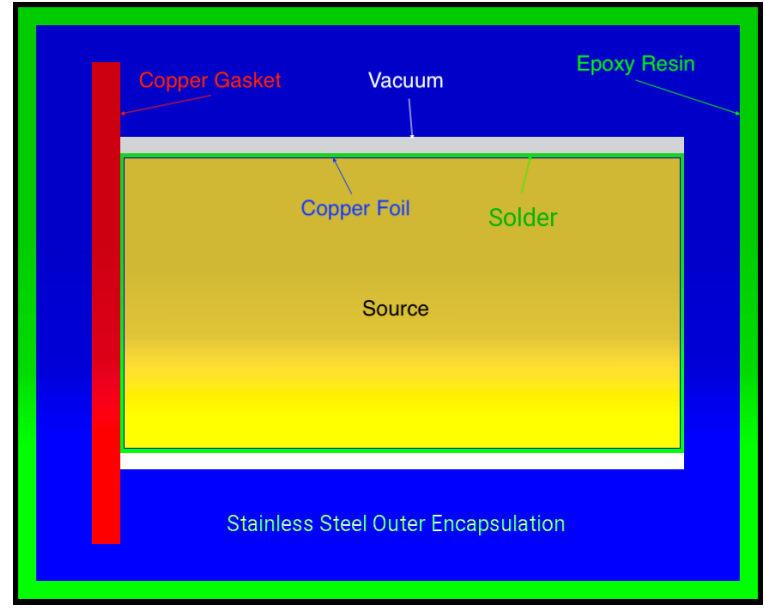

Figure 7.7: The simulated encapsulated AmBe source.

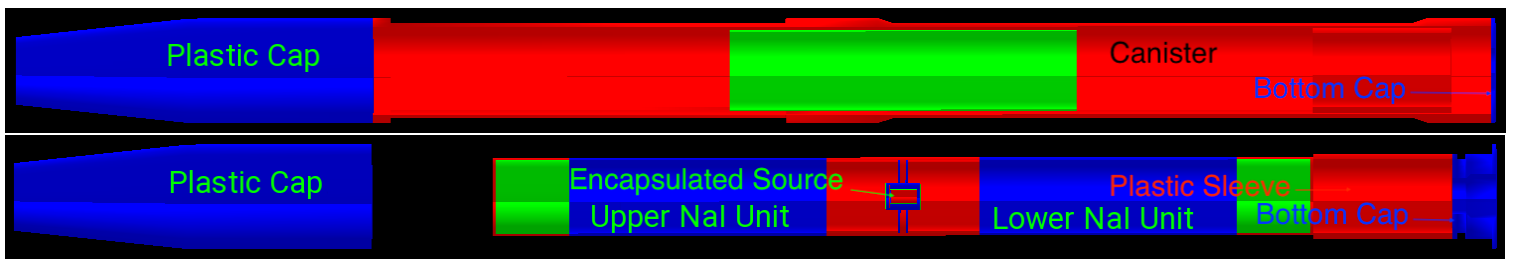

Figure 7.8: Top: The full source deployment geometry as implemented in the Monte Carlo simulation. Bottom: The source deployment geometry with the stainless steel canister and sleeve removed to show the inner components. 

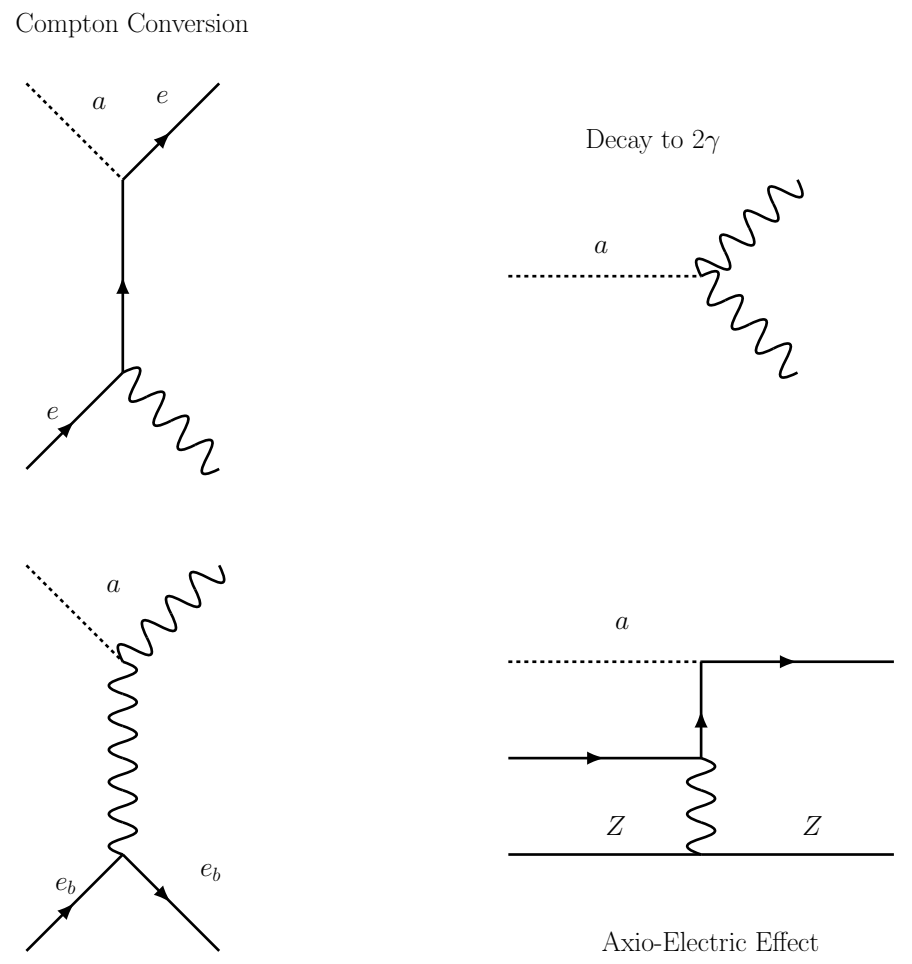

Axio-Electric Effect

\section{Inverse Primakoy}

Figure 7.9: The four axion interaction cross sections that DEAP-3600 may be sensitive to in the context of a $5.5 \mathrm{MeV}$ solar axion search. 


\section{Axion Decay MC Model}

The axion decay generator required the implementation of a relativistic model representing the distribution of energy between the two $\gamma$ rays. This is important because the energy of a $\gamma$ ray within the LAr determines the fraction of its energy that will be fully deposited within the LAr.

The decay $\gamma$ momenta are equal and opposite in the axion rest frame, with the magnitude of each momentum equal to half of the axion mass. The $\gamma$ rays are also emitted in random directions in the axion rest frame. When a relativistic boost is performed, the now decayed axion's kinetic energy will boost the $\gamma$ momenta in the direction of the axion in the lab frame.

The $\gamma$ velocity vector in the lab frame is calculated from its velocity vector in the axion rest frame. Since the $\gamma$ speed is, of course, unchanged, it was necessary to separately boost the $\gamma$ energy to the lab frame. The result was that events in which the $\gamma$ rays were emitted along the axion axis of motion showed the greatest difference between the $\gamma$ energies, while events in which the $\gamma$ rays were emitted perpendicular to the axion direction of motion yielded $\gamma$ rays that were more similar in energy.

\section{Compton Conversion Model}

In order to find the energy of the photon emitted under a Compton conversion interaction as a function of the axion mass, one needs to consider the Compton conversion differential cross section. This cross section is given in [51] as:

$$
\frac{d \sigma}{d \Omega}=\frac{\alpha \alpha_{A}}{2 m_{e}^{2}} \frac{E_{\gamma}}{p_{A}}\left[1+\frac{4 m_{e}^{2} E_{\gamma}^{2}}{y^{2}}-\frac{4 m_{e} E_{\gamma}}{y}-\frac{4 m_{A}^{2} p_{A}^{2} m_{e} E_{\gamma}}{y^{3}} \sin ^{2} \theta\right],
$$

where $\alpha$ is the fine structure constant, $\alpha_{a}=g_{A e}^{2} / 4 \pi, y=2 m_{e} E_{A}+m_{A}^{2}, \theta$ is the angle between the incoming axion and the outgoing $\gamma, E_{A}$ is the axion energy, $m_{e}$ is the electron mass, $p_{A}$ is the axion momentum, $g_{A e}$ is the axion-electron coupling 
constant, and $E_{\gamma}=y / 2\left(m_{e}+E_{A}-p_{A} \cos \theta\right)$ is the outgoing photon energy. The total cross section can be found by integrating eq. 7.1, and is given in eq. 2.13. In solving for $E_{\gamma}$, the only relevant unknown is $\theta$. $\theta$ is also the only unknown in eq. 7.1 . Note that eq. 7.1 can be thought of as a probability density function (pdf), with the normalization given by its integral in eq. 2.13. For the purposes of Monte Carlo simulations, the $\theta$ value was sampled from this pdf and used to compute the photon energy. The remaining axion energy goes to the ionized electron. Since electrons have an extremely short mean free path in liquid argon, the additional angle between the outgoing electron and the outgoing photon was ignored.

\subsection{Processing Simulation Output}

After the generation of events in the Monte Carlo, extensive processing was required to prepare them for use in the detector calibration and axion search fits. This processing involved extracting information from the Monte Carlo concerning the deposition of energy within the LAr, extracting information concerning the interactions within the simulation, processing the energy deposits into simulated detector triggers, and labelling those triggers based on which $\gamma$ lines were produced in the interaction that was primarily responsible for the trigger.

\subsubsection{Extracting Physics Process and Isotope Information}

The Monte Carlo saves the particle tracking information for all particles in the simulation at the Monte Carlo step level. This makes it possible to extract a high level of detail from the simulations. The simulations typically start with one to three primary particles. Using AmBe MC as an example, these particles include the neutron and up to two $\gamma$ particles that are produced simultaneously by the AmBe source. These particles can deposit energy directly in the LAr. But there is also a high chance that 
they will interact with some other component in the detector and produce secondary particles. The secondary particles can also interact directly with the LAr and can generate secondary particles of their own, and so on. Since all of the tracking information is recorded in the simulation output, it is possible to trace back the lineage of a particle all the way to one of the original primary particles. This makes it possible to label the energy depositions according to the primary particle that was ultimately responsible.

In the case where the primary particle responsible for an energy deposition was a neutron, additional information concerning the neutron interaction that spawned the secondary particles is extracted. All neutron interactions in the simulation occur according to a nuclear physics model whose parameters are set according to the specific isotopes present in the volume in which the neutron is travelling in a given step. Based on the cross sections of these isotopes, the simulation software determines whether an interaction will occur in a step. Once the software determines that an interaction should occur (through the use of a random variable), it proceeds to apply the portion of the nuclear physics model that describes the interaction. These models are incomplete, however, and, as will be discussed in Section 7.6, the output of the simulations showed major discrepancies with the data. To facilitate applying corrections to that output, the proton number (Z) and nucleon number (A) were extracted directly from the GEANT4 neutron interaction and included in the MC output, along with all the other variables necessary for downstream processing and validation. A list of the most important variables is given below. These variables are recorded for every step that deposits energy in the LAr.

- mc_id: The Monte Carlo event number

- edep: The amount of energy deposited in the LAr during this step

- dep_x, dep_y, dep_z: The location of the step within the LAr 
- process: The process that the primary particle underwent when it created the secondary chain of particles that led to the creation of the particle that is currently depositing energy in the LAr

- primary PDG: The ID of the primary particle that is ultimately responsible for this energy deposition according to the PDG MC particle ID convention

- edep_time: The time of the current step

- $\mathbf{Z}$ : The proton number of the isotope with which the primary particle interacted

- A: The nucleon number of the isotope with which the primary particle interacted

- vtx_time: The time at which the primary particle interacted

\subsubsection{Processing Energy Depositions into Simulated Triggers}

Once all the prerequisite variables have been extracted from the simulation, the next step is to use them to reconstruct triggers within the detector. The first step is to sort the energy deposits by time for each MC event. The time of the first deposit determines the start of the simulated trigger, which continues for the following $13.5 \mu \mathrm{s}$, matching the size of the event window after a real detector trigger. At the end of this time window, any remaining steps in the event will form additional triggers. In practice, this situation is quite rare, however.

In addition to the energy of a trigger, the position is recorded as well. This is recorded as the weighted average of energy depositions, according to the following equation:

$$
R_{j}=\frac{\sum_{i=1}^{N_{\text {step }}} e d e p_{i} \cdot d e p_{j i}}{e d e p_{\text {total }}}
$$

where $e d e p_{i}$ is the energy deposited in the LAr in MC step $i, d e p_{i j}$ is the location of step $i$, and the index, $j$, represents the $\mathrm{x}, \mathrm{y}$, or $\mathrm{z}$ coordinate. 


\subsubsection{Monte Carlo Neutron Capture $\gamma$ Spectrum}

For the purpose of performing corrections to a given neutron capture $\gamma$ spectrum, it was necessary to extract the spectrum from the simulation. This was done separately from the above mentioned processing, as it is not directly related to the simulated triggers or to the deposition of energy within the LAr. The simulation handles the production and propagation of the $\gamma$ particles and records the energy they deposit in the LAr. The spectra used internally by the GEANT4 simulation are reconstructed by storing the energies of all $\gamma$ particles produced by a neutron capture or inelastic scatter process and recording the $\mathrm{Z}$ and $\mathrm{A}$ of the isotope that the neutron interacted with and whether the interaction was a capture or scatter. This is done for all MC events generated, allowing the spectrum used by the Monte Carlo to be inferred. In order to apply the corrections to the model, the vector containing the list of $\gamma$ particles produced in each interaction was added to the final output file containing the simulated triggered events, with the vector assigned to the trigger that the interaction was most responsible for, if any existed.

\subsection{Fitting}

\subsubsection{Energy response function}

As mentioned previously, the Monte Carlo was generated with the RAT optical model turned off to increase the statistics of the MC sample and due to discrepancies between the optical model and the data at these high electron-recoil energies. This was accomplished by setting the scintillation yield to 0 in the $\mathrm{MC}$ and only looking at the energy deposits within the LAr. To convert the simulated triggered event energy to an equivalent number of photo-electrons (PE), an energy response function was used. This response function consisted of a quadratic energy scale component and a 
Gaussian resolution function with a variance that was quadratic in PE. The energy scale component is given below:

$$
P E_{\text {mean }}=A \cdot E^{2}+B \cdot E+C,
$$

where $A, B$, and $C$ are empirical parameters representing the strength of each of the three terms and are free to vary in the fit. The detector resolution is represented by a Gaussian pdf with a variance given by:

$$
\sigma^{2}=r_{1} \cdot P E_{\text {mean }}^{2}+\left(1+r_{2}\right) \cdot P E_{\text {mean }},
$$

where $r_{1}$ and $r_{2}$ are empirical parameters that are free to vary in the fit. The linear coefficient $\left(1+r_{2}\right)$ was chosen for consistency with similar analyses by other collaborators. The full response function can be written as a Gaussian:

$$
P(q P E)=\frac{1}{\sqrt{\sigma^{2} 2 \pi}} e^{-\left(q P E-P E_{\text {mean }}\right)^{2} / 2 \sigma^{2}} .
$$

\subsubsection{Fit Overview}

The fitter software proceeds through several steps before, during, and after fitting. An overview of these steps is given below. More details on many of these steps will be given in the following sections.

- Load the data

- Generate the cuts

- Calculate the detector dead-time and live-time based on the sub-runs present in the input file

- Set the overall model scale according to the corrected live-time 
- Apply the light yield correction to the data that the fit will see

- Fill the data qPE histogram

- If running an Ambe fit, select events with weights to fill into the background histogram and correct their light yield

- Fill background histogram and subtract it from data histogram

- Initialize response function parameters by using locations of largest peaks in the spectrum or using an optical MC fit

- Fill the MC histograms for all fit components

- Start fitting method

- Save best fit parameters and refill the histograms before drawing the final fitted spectrum

An AmBe calibration fit was performed to constrain the energy response parameters, since the number of events in the physics fit was too low to properly constrain these parameters.

\subsubsection{BAT and MCMC fitting intro}

BAT (Bayesian Analysis Tool) is a toolkit for performing Bayesian fits and provides several functions to analysis the results. BAT has two major advantages for this work. The first is the ability to extract good approximations to the fully marginalized probability density functions (Bayesian posterior pdfs) for all parameters included in the fit. The second is the ability to include Bayesian priors on any parameter.

Bayesian fits use Bayes' theorem to obtain the probability density functions for all parameters:

$$
p(\theta \mid x, M)=\frac{L(x \mid \theta, M) \pi(\theta \mid M)}{\int L\left(x \mid \theta^{\prime}, M\right) \pi\left(\theta^{\prime} \mid M\right) d \theta^{\prime}} .
$$


Here $x$ is a set of data points (i.e., the data histogram), $\theta$ is a set of fit parameters, and $M$ represents the model excluding the parameter values. $L(x \mid \theta)$ is the likelihood function, and describes the conditional probability of seeing the data $x$ given some set of parameter values $\theta . \pi(\theta)$ represents the prior knowledge of the "true" parameter values in the form of a probability density function, and is referred to as the prior probability density function. $p(\theta \mid x)$ is referred to as the posterior probability density function and expresses the conditional probability that the parameters being estimated by the fit are equal to some set of values $\theta$, given both the data $x$ and the prior knowledge $\pi(\theta)$ that was included in the fit. The power of Bayes' theorem is, therefore, the ability to update our knowledge of the true values of sets of parameters by combining new and prior data.

What is often desired is the marginal probability density function for each parameter, referred to as the marginalized posterior. This gives a probability density function for each parameter where all the correlations with all other parameters have been taken into account. The posterior, however, is a joint pdf of all parameters. In general, a marginal pdf can be found by evaluating:

$$
p_{\theta_{i}}\left(\theta_{i}\right)=\int_{-\infty}^{\infty} p\left(\theta_{1}, \theta_{2}, \ldots, \theta_{N} \mid x\right) \prod_{j \neq i}^{N} d \theta_{j}
$$

Evaluating this integral is not computationally practical when dealing with large numbers of parameters. However, a good, unbiased approximation is possible using Markov Chain Monte Carlo (MCMC) methods. MCMC operates in principle by randomly choosing values for all free parameters and evaluating the likelihood from the posterior pdf. Based on the likelihood evaluated from the last set of values, the new set of values is accepted or rejected. If the new set is rejected, the old set is reused. The parameter values are stored, typically in histograms. Assuming the Markov chain has run long enough to no longer be sensitive to its initial point, the 
parameter values stored in these iterations of the Markov chain serve to approximate the marginalized pdfs of all the parameters.

BAT uses the Metropolis-Hastings algorithm to perform this marginalization. Once a new set of parameters $\tilde{\theta}$ has been proposed (at random), the algorithm decides whether to accept or reject the new set by evaluating:

$$
u<\frac{p(\tilde{\theta} \mid x)}{p\left(\theta_{t-1} \mid x\right)}
$$

where $u$ is a uniform random variable between $[0,1]$ and $\theta_{t-1}$ is the previous set of parameter values. If eq. 7.8 is evaluated to be true, the new set is accepted and $\theta_{t}=\tilde{\theta}$. Otherwise the previous set of parameters is reused $\left(\theta_{t}=\theta_{t-1}\right)$. This chain of parameter values forms a Markov chain.

Until it converges to the global minimum, the Markov chain is dependant on its initial parameters, and will thus not give unbiased estimates of the marginalized posteriors except in the limit of infinite iterations. In practice, this means we want to run the Markov chain long enough that it loses any sensitivity to the initial parameter values. In BAT, this is done by running multiple chains in parallel until they all converge to similar sets of parameter values with similar variances. In BAT, the metric to determine whether convergence has been reached is the $R$-value 60 .

Before evaluating the estimates of the posteriors, BAT runs a pre-run to get the Markov chains to converge and to tune the proposal function that is responsible for proposing new sets of parameter values. The sets of parameters in the Markov chain prior to convergence are not valid for use in constructing the posterior function. In addition to the bias caused by the sensitivity to the starting parameters, tuning of the proposal function while running the Markov chain prevents it from properly approximating the posterior.

After the BAT pre-run has brought the Markov chains to convergence and has 
tuned the proposal function, a main run takes place which is responsible for running the Markov chain iterations that yield the approximated marginalized posteriors. The pre-run can be much longer than the main run in cases where the joint posterior is complex, such as in this work.

\section{Importance of parameter ranges}

It is not possible to draw random parameter values from an unbounded range of numbers. Ranges thus need to be set for each parameter. If these ranges are too large, the marginalized posterior pdfs will have very poor resolution. If they are too small, the posteriors will be cut off. If they are only slightly too large, the Markov chains could take a very long time to explore the large number of local minima before eventually converging, and may not converge at all. These problems are only made more severe when dealing with a large number of correlated parameters and very large datasets.

The most effective technique for setting these ranges was found to be:

- Use Minuit (the fitting package included in ROOT) to get an estimate of the best fit parameters (typically falls in a local minimum)

- Manually set large ranges for each of the fit parameters

- Run BAT for a small number of pre-run iterations (250,000 iterations was typical) and a long enough main run (typically 150,000 iterations) to get a good sense for the regions within the ranges that are favoured by the Markov chains

- Examine the (poorly) approximated posteriors and shrink the ranges, keeping a generous padding on either side

- Repeat until good marginalized posteriors are produced 
This process, though quite time consuming, proved to be the most efficient way to get convergence when running BAT on the full AmBe dataset.

\section{Likelihood Function}

The data is sorted into $400 \mathrm{PE}$-wide $(60.4 \mathrm{keV}$, on average) bins for the purpose of fitting. The AmBe fit contains 150 bins, while the physics fit contains 100. Since the data was taken over a fixed period of time, the total event count follows a Poisson distribution, as does the count in each bin. The likelihood of observing our dataset is therefore given by:

$$
L(\theta \mid x)=\prod_{i=1}^{B} \frac{\lambda_{i}(\theta)^{x_{i}} e^{-\lambda_{i}(\theta)}}{x_{i} !},
$$

where $B$ is the number of bins in the fit range, $\lambda_{i}(\theta)$ is the model expectation value in bin $i$, and $x_{i}$ is the number of data counts in bin $i$.

Since the likelihood can often take on very small values, the log-likelihood, which shares the same minimum, is typically used when performing a fit. In this case, the log-likelihood function is given by:

$$
\log L(\theta \mid x)=\sum_{i=1}^{B}\left[x_{i} \log \lambda_{i}(\theta)-\lambda_{i}(\theta)-\log \left(x_{i} !\right)\right]
$$

If a bin contains a large number of counts $x_{i}$ and if the observed number of counts is close to the expectation value $\lambda$ from the model, the Poisson distribution can be approximated as a Gaussian. The default threshold in BAT is to use the Gaussian approximation for bins with a model expectation of more than 899 counts. Due to the high probability of the number of counts in a data bin being a large number of Poisson sigma away from the model expectation, this setting was preserved for this 
project. The Gaussian log-likelihood function is:

$$
\log L(\theta \mid x)=\sum_{i=1}^{B}\left[-\frac{\left(x_{i}-\lambda_{i}(\theta)\right)^{2}}{2 \lambda_{i}}-\frac{1}{2} \log \left(2 \pi \lambda_{i}(\theta)\right)\right]
$$

As mentioned above, the spectrum being fit to the data was generated with the help of Monte Carlo simulations. The finite statistics of these simulations can be significant if they are not sufficiently larger than the statistics in the data they are being fit to. The rule-of-thumb is that the MC model statistics should be at least a factor of 10 larger than the data statistics, otherwise the MC model uncertainties need to be taken into account. In this work, the AmBe calibration dataset contains over $23,000,000$ triggers in the fit range. Since the number of triggers in the fit range per neutron generated is only $0.5 \%$, one would need roughly $4.5 \times 10^{10} \mathrm{MC}$ AmBe events to get a factor of 10 greater MC statistics, which is impractical. It was, therefore, necessary to include the MC statistical uncertainties in the likelihood function.

The value $\lambda_{i}(\theta)$ in each model bin is equal to the sum of the bin values $\eta_{i, j}$ of the several spectral components after being scaled by their fit normalization parameter $s_{i}$ :

$$
\lambda_{i}(\theta)=\sum_{j=1}^{C} \eta_{i, j}(\theta) s_{j}(\theta) .
$$

The values $\eta_{i, j}$ are computed by applying the response function to the list of MC event energies. After applying eq. 7.3 to the list of summed energies from each triggered MC event, the resulting $P E_{\text {mean }}$ and the corresponding $\sigma$ from eq. 7.4 are used to compute a Gaussian pdf for each event. The value $\eta_{i, j}$ in each bin is thus made up of a sum of weights $<1$. This means that the final MC model value $\lambda_{i}$ in each bin is the sum of a very large number of small weight values. According to the central limit theorem, a Gaussian should thus be an acceptable approximation for the uncertainty in each bin. 
The uncertainty $\sigma_{\eta_{i, j}}$ in each bin is computed by taking the sum of the weights squared in each bin:

$$
\sigma_{\eta_{i, j}}(\theta)=\sqrt{\sum_{k=1}^{n} w_{i, j, k}^{2}(\theta)}
$$

where $n$ is the total number of simulated MC triggers. The weights are calculated from the Gaussian pdfs calculated according to the response function. Since the bin has a finite width, an integral was performed using the Gaussian cumulative distribution function (cdf):

$$
w_{i, j, k}(\theta)=\operatorname{erf}\left(\frac{b_{k}-P E_{\text {mean }}(\theta)}{\sigma(\theta) \sqrt{2}}\right) / 2-\operatorname{erf}\left(\frac{a_{k}-P E_{\text {mean }}(\theta)}{\sigma(\theta) \sqrt{2}}\right) / 2,
$$

where $a_{k}$ and $b_{k}$ are the lower and upper bin edge, respectively, and $\sigma(\theta)$ is the square root of the result of eq. 7.4. The total MC uncertainty per bin after applying the MC component normalizations is calculated through standard error propagation:

$$
\sigma_{\lambda_{i}}(\theta)=\sqrt{\sum_{j=1}^{C} \sigma_{\eta_{i, j}}^{2}(\theta) s_{j}^{2}(\theta)}
$$

To propagate $\sigma_{\lambda_{i}}(\theta)$ through the likelihood functions, one needs to combine the likelihood functions with the Gaussian distribution that describes the uncertainties. If the likelihood function is also a Gaussian, the uncertainties can be added in quadrature. The Gaussian log-likelihood function eq. 7.11 simply becomes:

$$
\log L(\theta \mid x)=\sum_{i=1}^{B}\left[-\frac{\left(x_{i}-\lambda_{i}(\theta)\right)^{2}}{2\left(\lambda_{i}(\theta)+\sigma_{\lambda_{i}}^{2}(\theta)\right)}-\frac{1}{2} \log \left(2 \pi\left(\lambda_{i}(\theta)+\sigma_{\lambda_{i}}^{2}(\theta)\right)\right)\right]
$$

For the case of a Poisson likelihood function, the following must be evaluated 
(omitting the dependant variables, $\theta$, for readability):

$$
\begin{aligned}
P_{\text {conv }} & =\sum_{i=1}^{B}\left[\int_{0}^{\infty} P_{\text {Poisson }}\left(x_{i} \mid \lambda\right) P_{\text {Gaus }}\left(\lambda \mid \lambda_{i}, \sigma_{\lambda_{i}}\right) d \lambda\right] \\
& =\sum_{i=1}^{B}\left[\frac{1}{x_{i} !} \frac{1}{\sigma_{\lambda_{i}} \sqrt{2 \pi}} \int_{0}^{\infty} e^{-\frac{1}{2}\left(\frac{\lambda-\lambda_{i}}{\sigma_{\lambda_{i}}}\right)^{2}} \lambda^{x_{i}} e^{-\lambda} d \lambda\right] .
\end{aligned}
$$

Note that the lower integral bound starts at 0 . This is because a Poisson distribution is only valid for $\lambda>0$. The Gaussian component, therefore, has to be sufficiently greater than 0 to be a valid estimate of the uncertainty. After some algebra, this becomes:

$$
P_{\text {conv }}=\sum_{i=1}^{B}\left[\frac{1}{x_{i} !} \frac{1}{\sigma_{\lambda_{i}} \sqrt{2 \pi}} e^{-\lambda_{i}+\sigma_{\lambda_{i}}^{2} / 2} \int_{0}^{\infty} e^{-\frac{1}{2}\left(\frac{\lambda-\left(\lambda_{i}-\sigma_{\lambda_{i}}^{2}\right)}{\sigma_{\lambda_{i}}}\right)^{2}} \lambda^{x_{i}} d \lambda\right]
$$

This integral can only be solved if the lower bound is $-\infty$. This is valid if Gaussian component of the integrand is sufficiently far from 0 . Otherwise, it is possible for the function to take on negative values that will render this a poor approximation for the combined uncertainty. If the mean of the Gaussian component is at least 2 standard deviations above $0,2.5 \%$ of the function area will be below 0 . The condition for the validity of this new likelihood function is then $\lambda_{i}>\sigma_{\lambda_{i}}\left(\sigma_{\lambda_{i}}+2\right)$. Fortunately, almost no cases were encountered during fitting where this condition was not met (the software prints a warning if it isn't met). We can then perform the integral over $(-\infty, \infty)$ to get:

$$
L=P_{\text {conv }}=\sum_{i=1}^{B}\left[\frac{\sigma_{\lambda_{i}} x_{i}}{\sqrt{2}} \frac{e^{-\lambda_{i}+\sigma_{\lambda_{i}}^{2} / 2}}{x_{i} !} \frac{H_{x_{i}}(j \beta)}{j^{x_{i}}}\right]
$$

where $H_{n}(x)$ are the Hermite polynomials, $\beta=\left(\lambda-\sigma_{\lambda_{i}}^{2}\right) / \sqrt{2} \lambda_{i}$, and $j$ is the imaginary 
number. The final log-likelihood is:

$$
\log L(\theta \mid x)=\sum_{i=1}^{B}\left[\sigma_{\lambda_{i}}^{2}(\theta) / 2-\lambda_{i}(\theta)-\log \left(x_{i} !\right)+\log \left(\left(\frac{\sigma_{\lambda_{i}}(\theta)}{\sqrt{2} j}\right)^{x_{i}} H_{x_{i}}(j \beta)\right)\right]
$$

This result was fully implemented in the fitting code. This likelihood function was used whenever $\lambda_{i} \leq 899$. If $\lambda_{i}>899$, the Gaussian likelihood function (eq. 7.16) was used.

\section{Extracting Results from the Likelihoods}

The most important output of BAT is a set of $1 \mathrm{D}$ and $2 \mathrm{D}$ marginalized posterior histograms for all parameters and all combinations of 2 parameters. The posteriors were used to set the best fit parameter values and their uncertainties. The best fit parameters were set to be equal to the modes of the posteriors. The uncertainties were calculated by integrating the posteriors. The integration started at the mode, and added the next largest bin until the desired confidence level was reached. If the bin at either edge of the histogram was included in the integration, the parameter value was taken to be a limit.

\section{Plot Residuals}

The final fit plots (after all corrections) include residuals to give a sense of the level of agreement between the model and the data. They include the fit uncertainties, statistical model uncertainties, and the Poisson statistical fluctuations in the data. The fit uncertainties are computed by recording the model values in each bin after each iteration of the Markov chain. These histograms form an approximation to the marginalized posterior pdf of the model value in each bin. The model (MC) statistical uncertainty gets factored in by performing a numerical integral of the product of the Gaussian with the marginalized posterior, with the distribution of Gaussian means 
given by the posterior. The resulting histogram is integrated with a Poisson to obtain the final model pdf for the bin. The overall operation can be summarized as:

$$
f_{i}\left(x_{i}\right)=\int_{0}^{\infty} P_{\text {Poisson }}\left(x_{i} \mid \lambda\right) P_{\text {Gaus }}\left(\lambda \mid \lambda_{i}, \sigma_{\lambda_{i}}\right) P_{\text {model }}\left(\lambda_{i}\right) d \lambda_{i} d \lambda
$$

This final pdf is integrated around the median to compute the $68.2 \%, 95.4 \%$, and 99.7\% quantiles, which are plotted in the residuals plot.

\subsection{Corrections to Simulated Model}

It was quickly discovered that the model as simulated by RAT and GEANT4 didn't match the data well. The fit result is shown in Figure 7.10. It was determined that the source of the discrepancy was likely the neutron physics model in GEANT4. The neutron capture cross sections used by GEANT4 were compared with the ENDS (Evaluated Nuclear Data Sheet) tables available at [61] and [62], and it was found that they matched quite well. This left the neutron propagation model as a likely source of the discrepancy, as this would greatly affect the number of neutrons that capture on each component of the detector.

\subsubsection{Separating Model into Capture- $\gamma$ Spectral Components}

When a neutron capture occurs, the $\gamma$-rays that are emitted carry away the binding energy of the neutron and any excess kinetic energy. Since the neutron capture cross section only becomes significant once the neutrons have lost enough energy to become thermal (around $0.025 \mathrm{eV}$ ), the kinetic energy of the neutron upon capture is typically negligible. Each isotope will thus emit a spectrum of $\gamma$-rays according to the binding energy of the additional neutron and the nuclear energy levels of the new isotope.

From Figure 7.10, it appears that the major $\gamma$ lines are being produced in the 

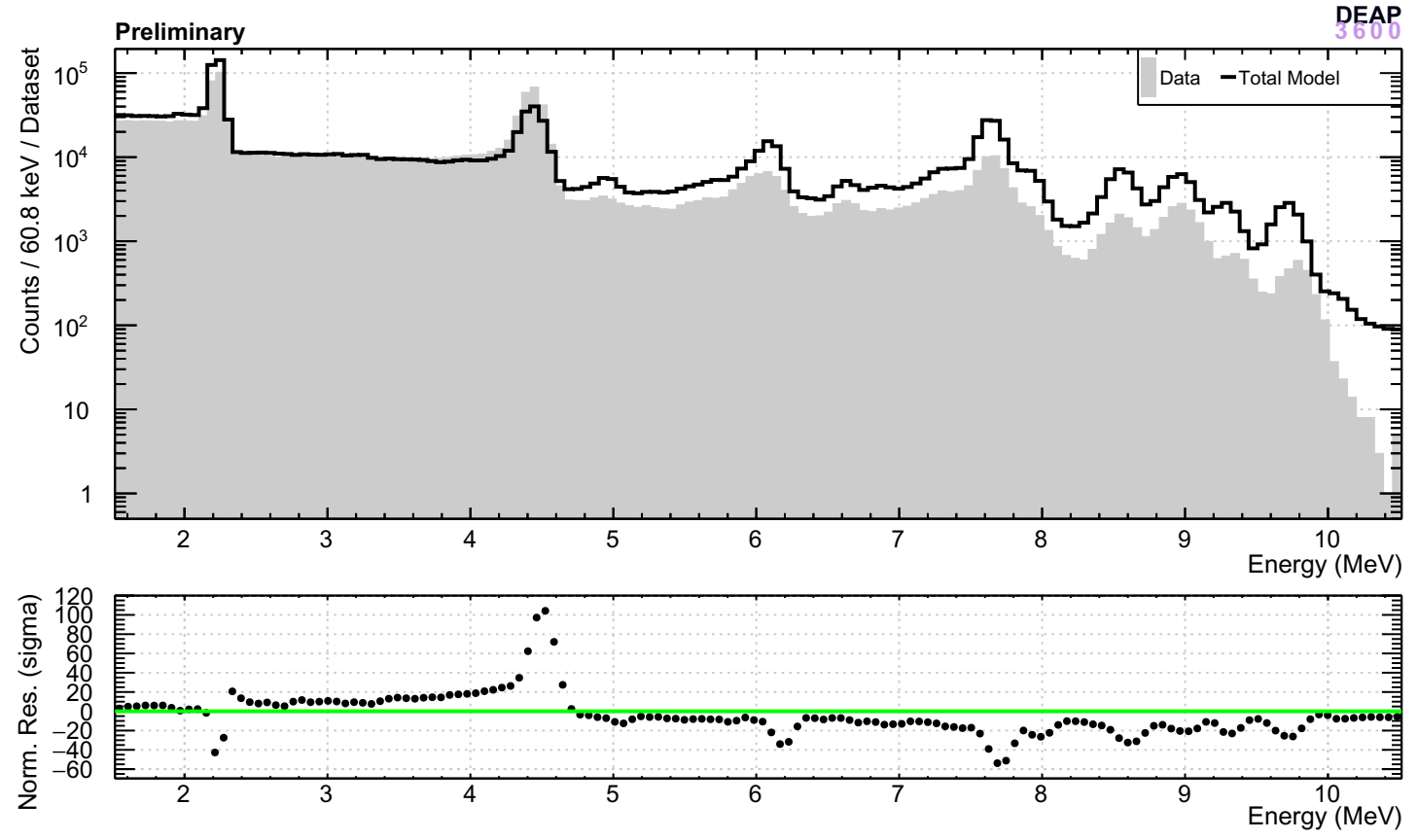

Figure 7.10: Fit of the AmBe MC model to a single AmBe calibration run (20714) before performing any corrections to the MC model or allowing individual capture- $\gamma$ spectra to float in the fit. Note that this fit was performed with Minuit, so the residuals plot only includes the statistical data and MC uncertainties. 

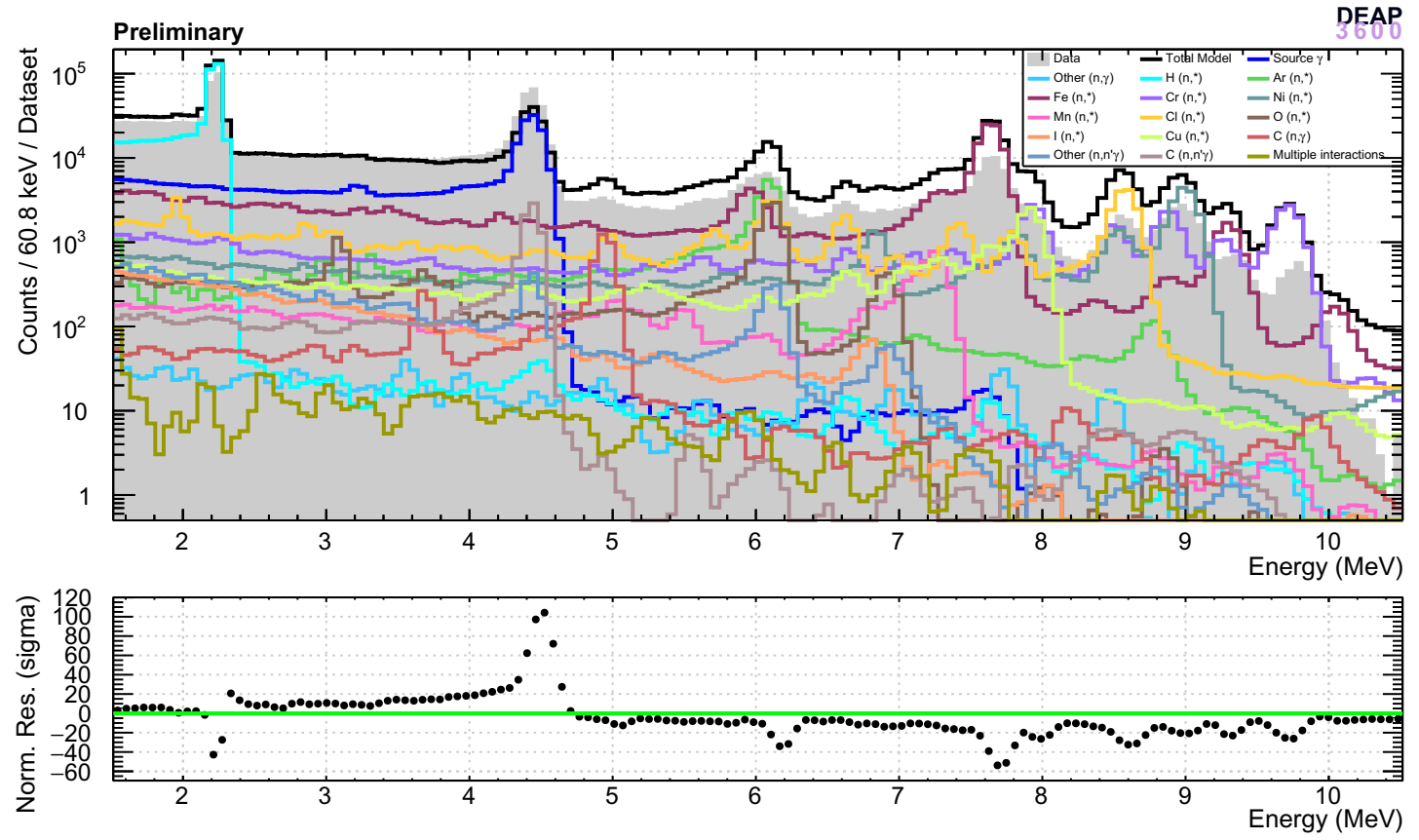

Figure 7.11: Same as Figure 7.10, but with the neutron capture- $\gamma$ spectra overlaid.

MC, but with the wrong amplitudes. To correct this, MC events were grouped into components based on the element that captured the neutron. These components can be seen in Figure 7.11. It was decided to group them by element rather than by isotope because grouping by isotope would create too many free parameters for the fit, and because the neutron capture cross sections appear to be correct in the MC, and so should produce a correct relative ratio of captures among the different isotopes present for each element. The normalizations of these components were allowed to vary in the fit. The resulting fit is shown in Figure 7.12. A summary of all components in the fit is given in table 7.1 .

\subsubsection{Corrections to the Neutron Capture- $\gamma$ Spectra}

Figure 7.12 shows great improvement over Figure 7.10 , but there are still some major discrepancies. The discrepancy at $6.6 \mathrm{MeV}$ is particularly interesting, as the only 


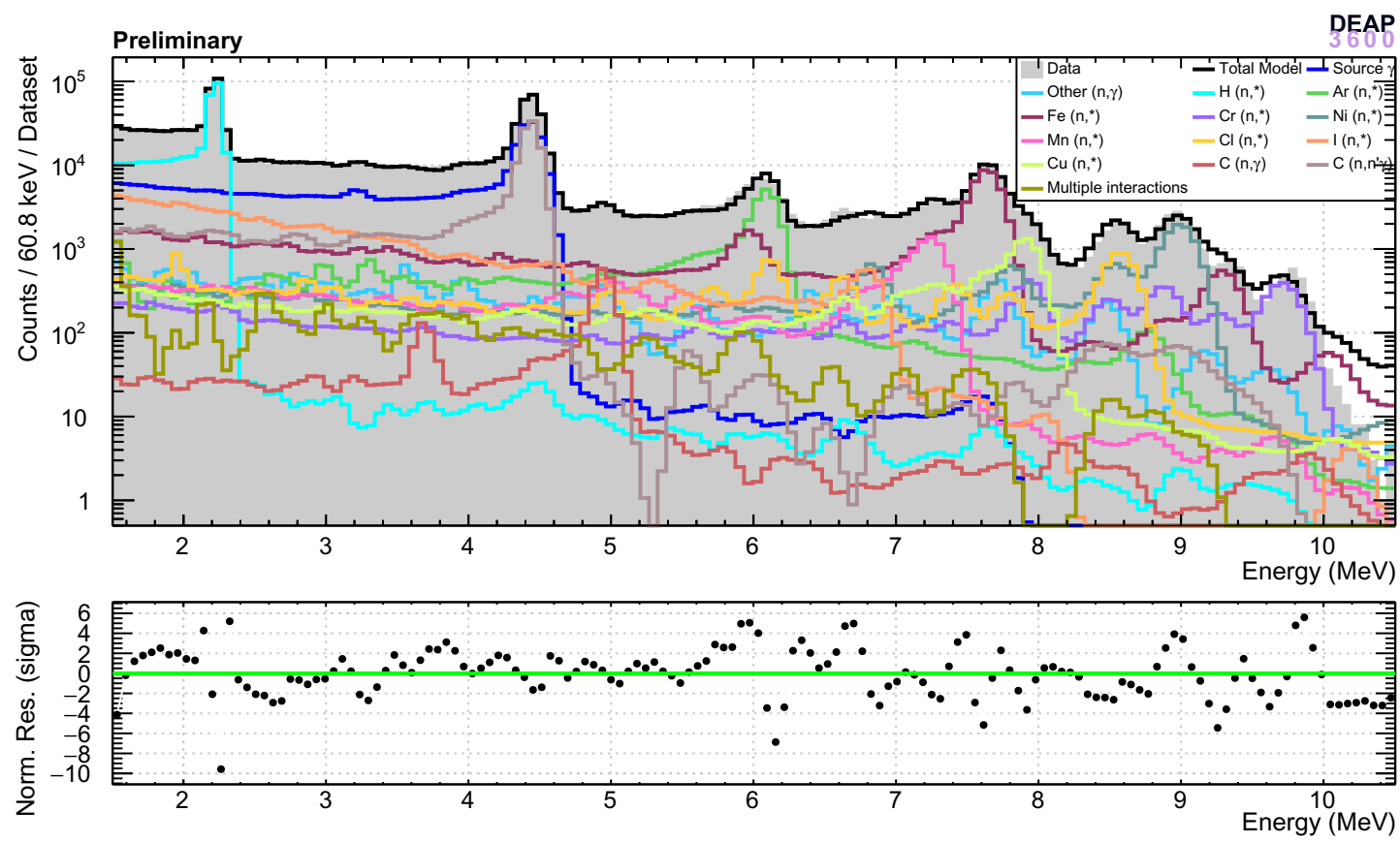

Figure 7.12: Fit of the AmBe MC model to a single AmBe calibration run (20714) with the neutron capture- $\gamma$ spectra grouped into components by element. The normalizations of the spectra were allowed to vary in the fit, but no further corrections were performed to the MC model. Note that this fit was performed with Minuit, so the residuals plot only includes the statistical data and MC uncertainties. Also note that the residual Y-axis scale is smaller than in previous plots. 

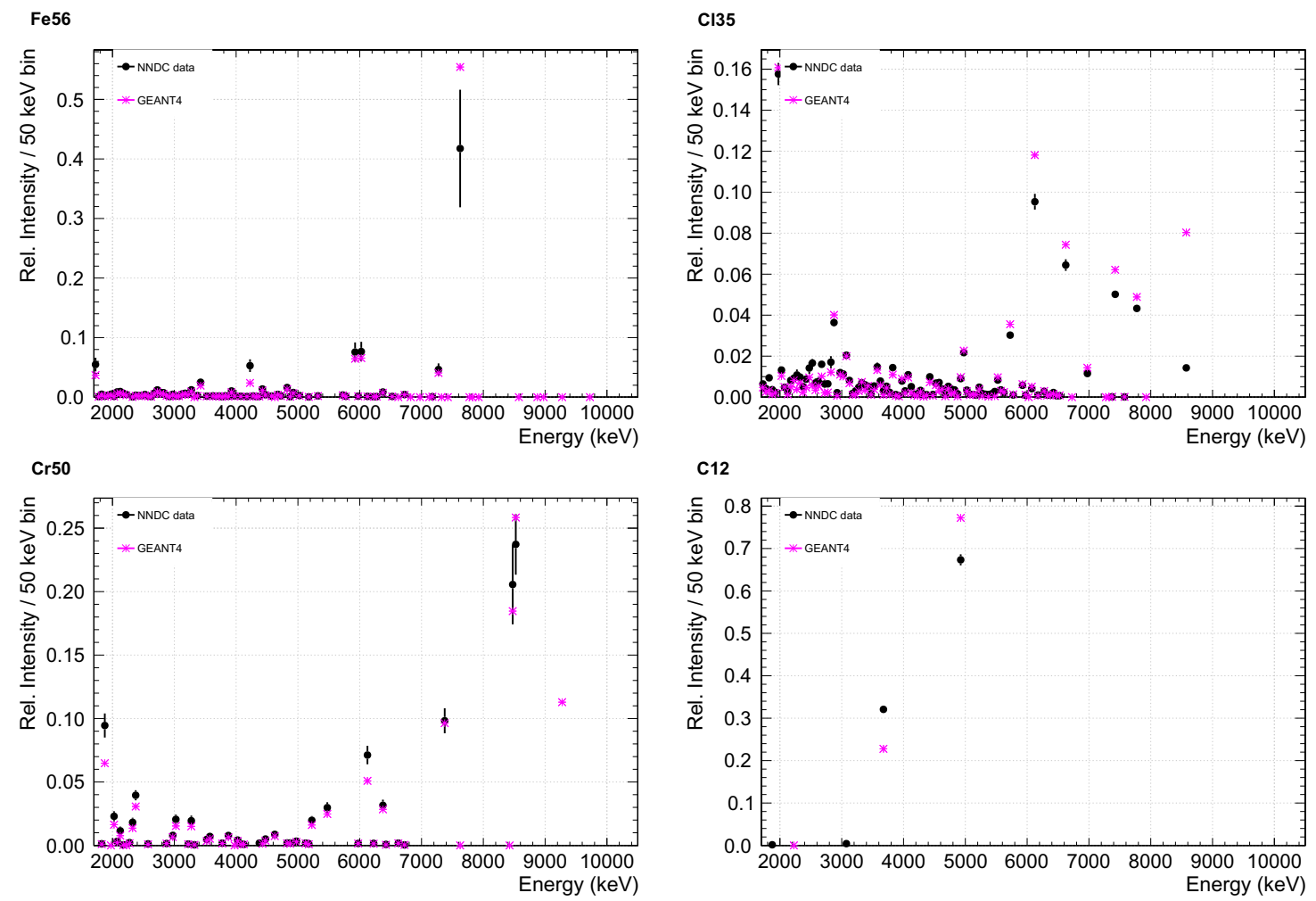

Figure 7.13: Comparisons of select neutron capture- $\gamma$ spectra as catalogued in ENDF tables and stored in [53 and [62 (black circles) and as produced by GEANT4.9.6 (magenta stars), illustrating some of the discrepancies between the two.

component capable of fitting that peak is chlorine. However, chlorine is already constrained from fitting any larger by the peak at $8.6 \mathrm{MeV}$. Nickel cannot be reduced to make room for chlorine at this peak, because it is also constrained by the peak at $9.0 \mathrm{MeV}$. This motivated a validation of the neutron capture- $\gamma$ spectra that was being produced by GEANT4.

As previously described in Section 7.4.3, the GEANT4 neutron capture- $\gamma$ spectra were inferred from the output of the simulations. These were compared with the spectra available in the online libraries [53]. Some examples of these comparisons are shown in Figure 7.13 .

These discrepancies are understood by the author as arising from the design of the 
GEANT4 neutron model. Due to a lack of reliable data, the correlations between $\gamma$ lines emitted during a neutron capture were not known well enough to be implemented into GEANT4.9.6 at the time of its development. Thus, in order to ensure that the total energy carried by the neutron capture- $\gamma \mathrm{s}$ is conserved on average, the neutron capture- $\gamma$ line amplitudes are allow to differ somewhat from the measured values. In addition, as can be seen in Figure 7.13, some $\gamma$ lines are produced at the Q-value of the neutron capture reaction, even though a single $\gamma$ might never be produced at that energy in reality. For an experiment that typically measures the total neutron capture- $\gamma$ energy emitted by a capture, this would not be a problem. This is not the case for DEAP-3600, however, where only one $\gamma$ per event is typically observed in the detector. It is, therefore, more important for us to correctly model the intensities of the $\gamma$ lines than to get the total average $\gamma$ energy to match the $\mathrm{Q}$-value.

Corrections were made to the neutron capture- $\gamma$ spectra by applying weights to events based on the presence of a particular $\gamma$ line. This made it possible to correctly model the capture- $\gamma$ spectra without requiring any changes to the simulated events themselves. A range of $\gamma$ energies was selected and events were weighted if a $\gamma$ was produced that fell in that range. The weight value was chosen by minimizing the residuals between the GEANT4 and NNDC spectra in Figure 7.13. Examples of corrected spectra are shown in Figure 7.14 .

To preserve the relative cross sections of various isotopes within a particular fit component, the fraction of events that were weighted was also recorded. A renormalization constant was computed for all events belonging to an isotope in which at least one $\gamma$ line was corrected. This constant was calculated according to:

$$
D=\frac{1}{1-\sum_{i=1}^{N_{w}} W_{i} \cdot F_{i}}
$$

where $D$ is the re-normalization constant for a particular isotope that multiplies every 

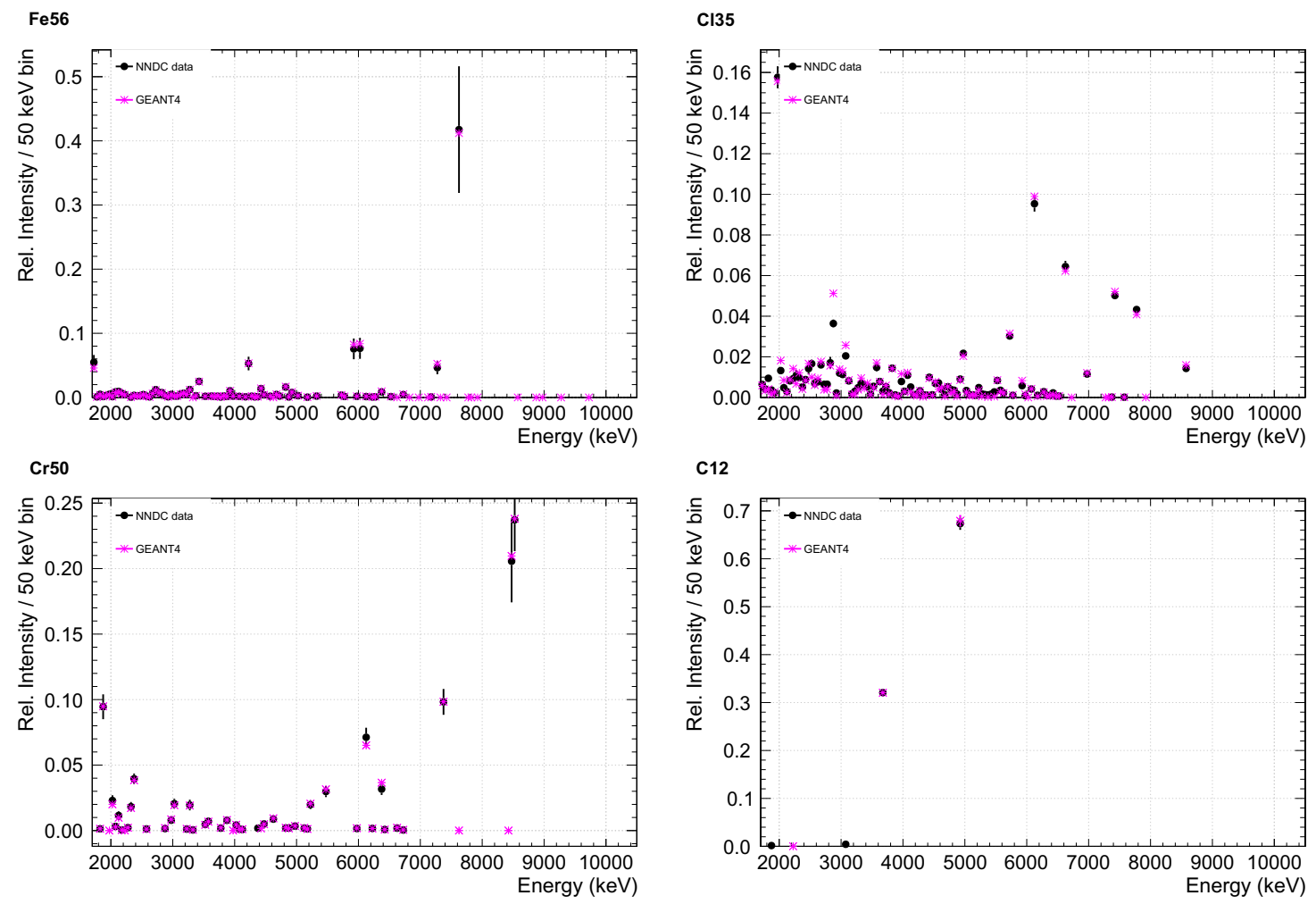

Figure 7.14: The same spectra from Figure 7.13 after correcting the spectra by weighting events based on the presence of $\gamma$ lines. 

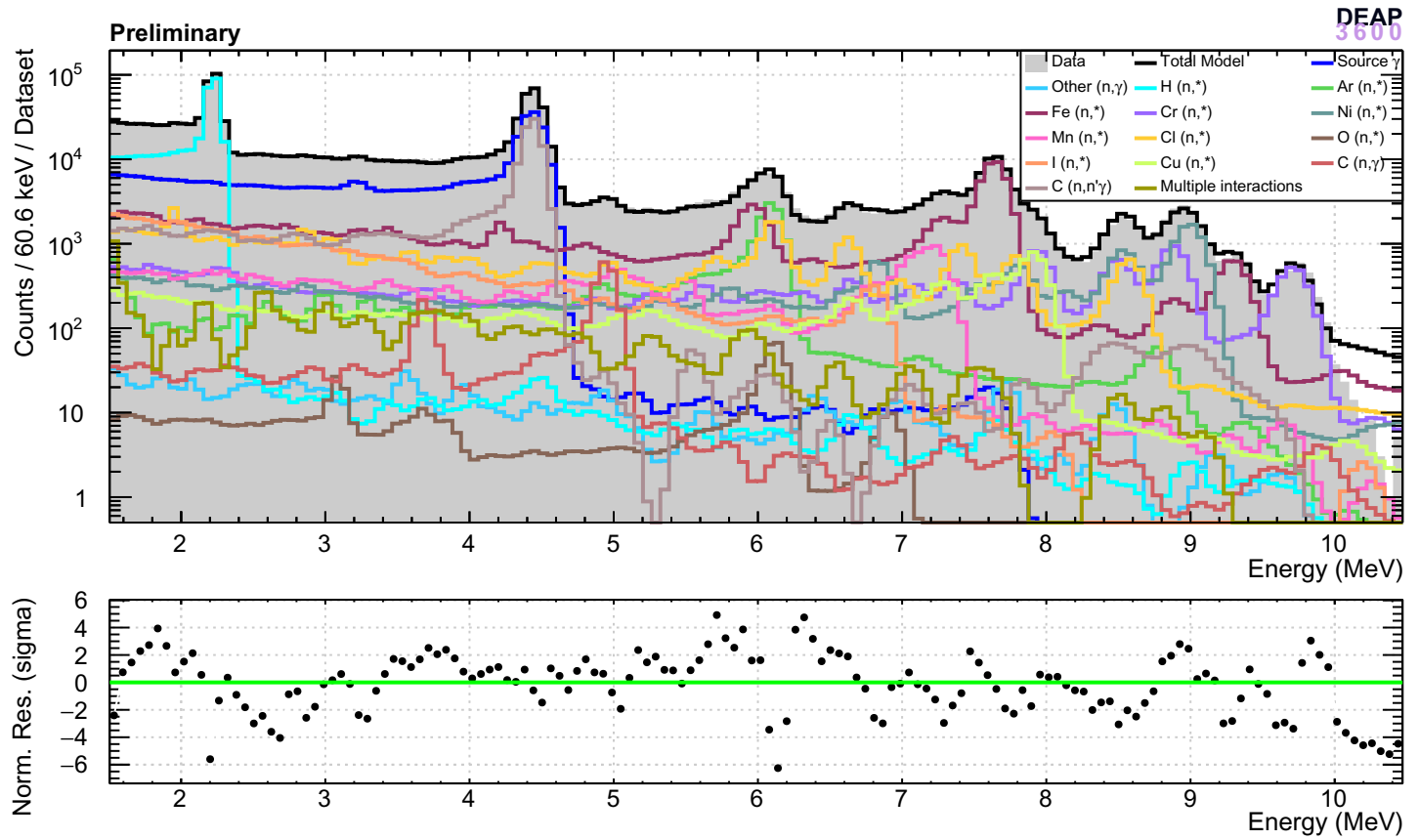

Figure 7.15: Fit of the AmBe MC model to a single AmBe calibration run (20714) with the neutron capture- $\gamma$ spectra free to float in the fit and corrections applied to the some of the $\gamma$ lines within the individual spectra. Note that this fit was performed with Minuit, so the residuals plot only includes the statistical data and $\mathrm{MC}$ uncertainties. Note that the residual Y-axis scale is smaller than in previous plots.

triggered event produced by a neutron capture on that isotope, $N_{w}$ is the total number of corrections applied to the capture spectrum, $W_{i}$ is a weighting factor, and $F_{i}$ is the fraction of neutron captures that produce a $\gamma$ in the range for which $W_{i}$ applies.

The resulting fit after applying these corrections is shown in Figure 7.15. The corrections were stored in a text file for easy modification.

\section{Spectral Systematic Uncertainties}

The online libraries themselves often list large uncertainties on the amplitude of certain capture- $\gamma$ lines. $\gamma$ lines containing large uncertainties were investigated as sources of systematic uncertainties in the fit. With some modifications to the code that was 

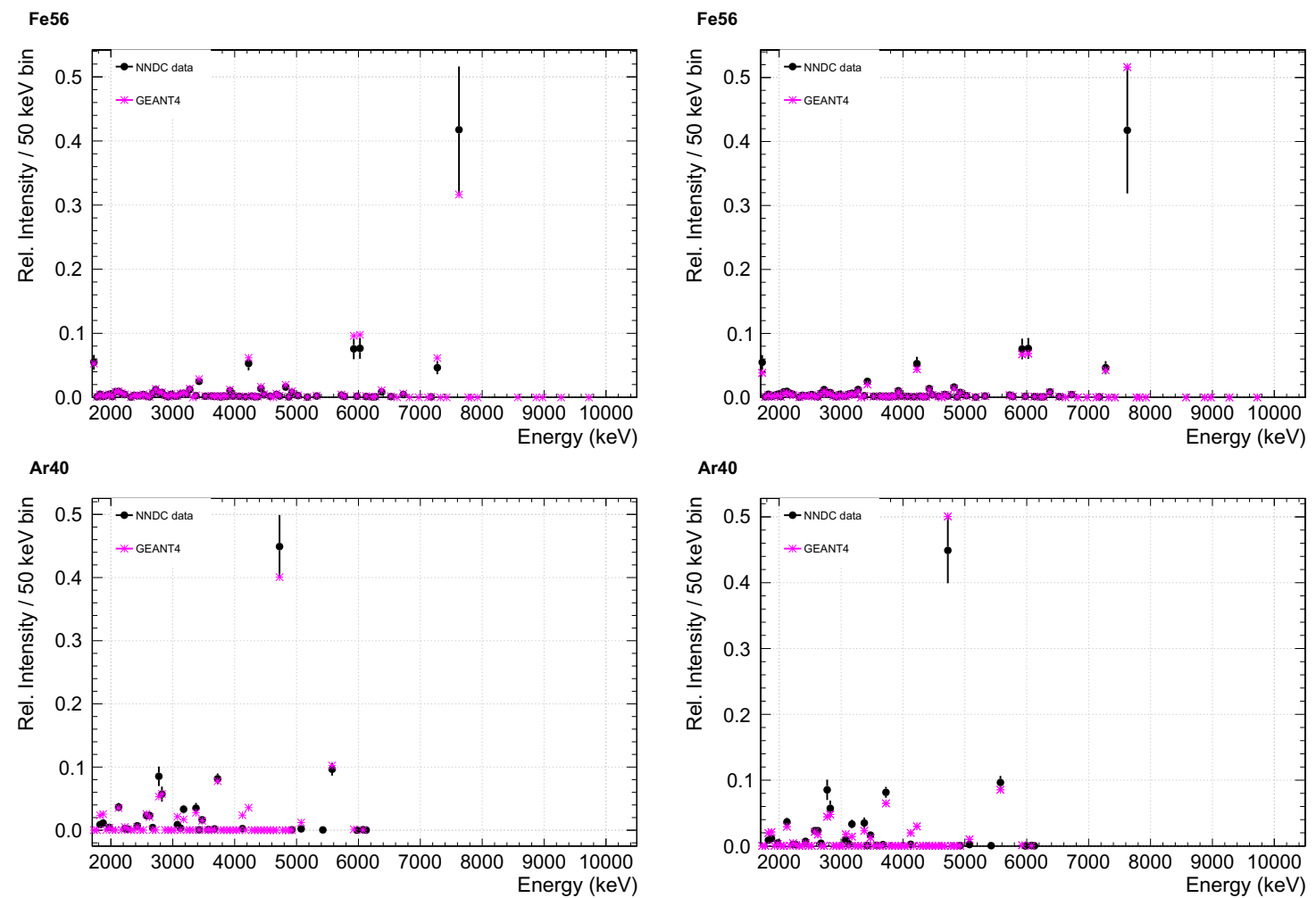

Figure 7.16: Above: An example illustrating the method used to compute the priors used in incorporating the uncertainty of spectral lines into the fit. The $7.6 \mathrm{MeV}$ neutron capture $\gamma$ line for ${ }^{56} \mathrm{Fe}$ is being varied in these plots. Below: Computing the priors for the $4.7 \mathrm{MeV}^{40} \mathrm{Ar} \gamma$ line.

used to calculate the weighting factor when correcting the GEANT4 spectra, systematic weighting factors were calculated such that the resulting $\mathrm{MC} \gamma$ line amplitude was at either end of the NNDC error bar for that $\gamma$ line. An example of this is shown in Figure 7.16. These weights describing the systematic uncertainty of the $\gamma$ spectral lines were also stored in a text file that was read by the fitter.

Within the fitter, the components containing $\gamma$ lines that were assigned to undergo a variation in the fit were split into events that contained a $\gamma$ line being varied, events from the same isotope without any $\gamma$ lines that were being varied, and events from other isotopes belonging to the same $\mathrm{MC}$ component. The overall component normalization was preserved, and an additional parameter was introduced describing 
the level of variation of a particular $\gamma$ line within its component. A Bayesian prior constrained this parameter based on the systematic weighting factors calculated as described above. These factors were taken to represent the standard deviations of a split normal distribution, which was set as the prior distribution for these $\gamma$ line amplitudes. By running the BAT fit with these new parameters free to vary, the spectral systematic uncertainties were incorporated directly into the marginalized posteriors of all the fit parameters.

There were far too many $\gamma$ lines with large error bars to allow the fitter to have any chance of converging. To reduce the number of $\gamma$ lines that were allowed to vary in the fit, simple Minuit fits were performed in which the $\gamma$ lines were fixed to plus or minus $1 \sigma$. If the resulting fit showed at least a 5\% change in the resulting $\chi^{2}$, or if it made a noticeable shift in the normalizations of the other parameters, it was considered significant enough to include in the fit. It was found that only $2 \gamma$ lines met this criteria - the $7.6 \mathrm{MeV}^{56} \mathrm{Fe} \gamma$ line and the $4.7 \mathrm{MeV}^{40} \mathrm{Ar} \gamma$ line.

\subsubsection{Energy Conservation in $\mathrm{MC}$}

As mentioned in the previous section, GEANT4.9.6 ensures that the average of the total energy emitted in the form of $\gamma$ rays in neutron capture interactions is equal to the Q-value. The energy is not equal to the Q-value on the individual event level, however. This makes it possible for triggered MC events to exist where the total amount of energy deposited in the LAr is greater than the Q-value (plus the typically negligible kinetic energy of the neutron) of the neutron interaction. This can be seen in Figure 7.15 above $10 \mathrm{MeV}$, where the model spectrum is several standard deviations greater than the data points. These are due to events where GEANT4 produces several $\gamma$ rays where the sum of the $\gamma$ energies is greater than the sum of Q-value of the reaction and the kinetic energy of the incident neutron. Fortunately, it is quite rare for several $\gamma$ rays to deposit energy within the LAr, making this effect 
negligible overall.

\subsection{Cuts and Data Corrections}

Several cuts and corrections were applied to the data prior to fitting.

\subsubsection{Cuts}

The following cuts were applied:

- $0.2>$ fprompt $>0.3$ (selects electron-recoil events)

- mblikelihoodR < 747 (radial cut)

- Standard low-level cuts (removes bad triggers)

- Standard pileup cuts

- fprompt < 0.3 \&\& fprompt > qPE. $4.31034 \times 10^{-7}+0.249828$

- deltat > 20000 (removes events within $20 \mu$ s of a previous trigger)

- clippedV1740Count==0 (removes events with unreliable saturation correction)

\section{Radial cut}

To reduce the neutron capture- $\gamma$ background and thus improve the sensitivity to a potential axion signal, a cut was applied to exclude events with a reconstructed position outside a $747 \mathrm{~mm}$ radius from the centre of the LAr volume. This value was chosen by maximizing the signal acceptance relative to the square root of the background:

$$
S=\frac{A_{e^{-}}}{\sqrt{A_{B}}}
$$




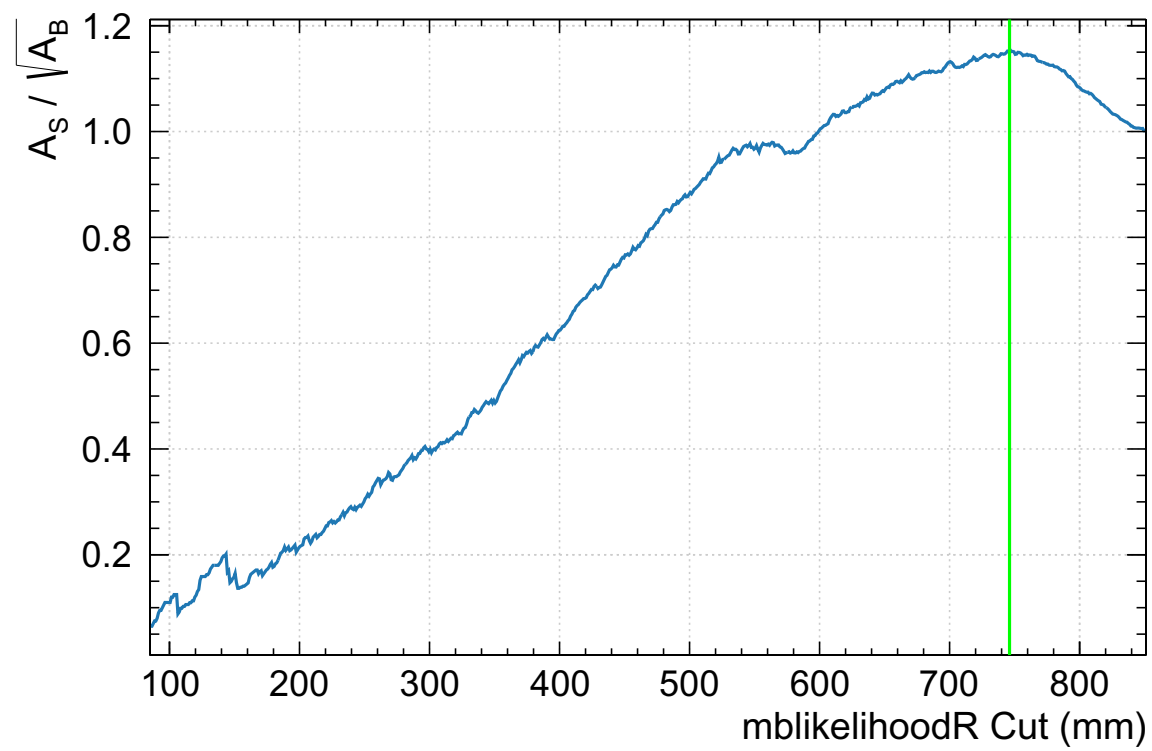

Figure 7.17: The 5.5 MeV electron signal sensitivity as quantified by eq. 7.23 as a function of an upper radial cut for events with 41,000 $<$ qPE $(\mathrm{MC})<42,000$. The point where the sensitivity is maximized is labelled by the green line.

where $A_{e^{-}}$is the acceptance of a $5.5 \mathrm{MeV}$ electron and $A_{B}$ is the background acceptance. A plot of eq. 7.23 as a function of an upper radial cut is shown in Figure 7.17. The optimal upper cut value was found to be $747 \mathrm{~mm}$. The acceptance of the uniformly distributed $5.5 \mathrm{MeV}$ electron interaction was $86.6 \pm 0.4($ stat $) \%$, while the neutron background acceptance was $57 \pm 2($ stat $) \%$. The MC events that this cut was tuned on were generated with the optical model turned on. The $5.5 \mathrm{MeV}$ signal events were generated specifically for this work, while the neutrons were generated by a collaborator using an older version of the MC software for use in the previous WIMP search analysis. The qPE range used for this measurement was selected by examining the qPE of $5.5 \mathrm{MeV}$ signal $\mathrm{MC}$ events where the full energy was deposited in the detector.

The same exercise was repeated for $5.5 \mathrm{MeV} \gamma$ signal events. The resulting sensitivity as a function of radial cut is shown in Figure 7.18. The optimal cut value 


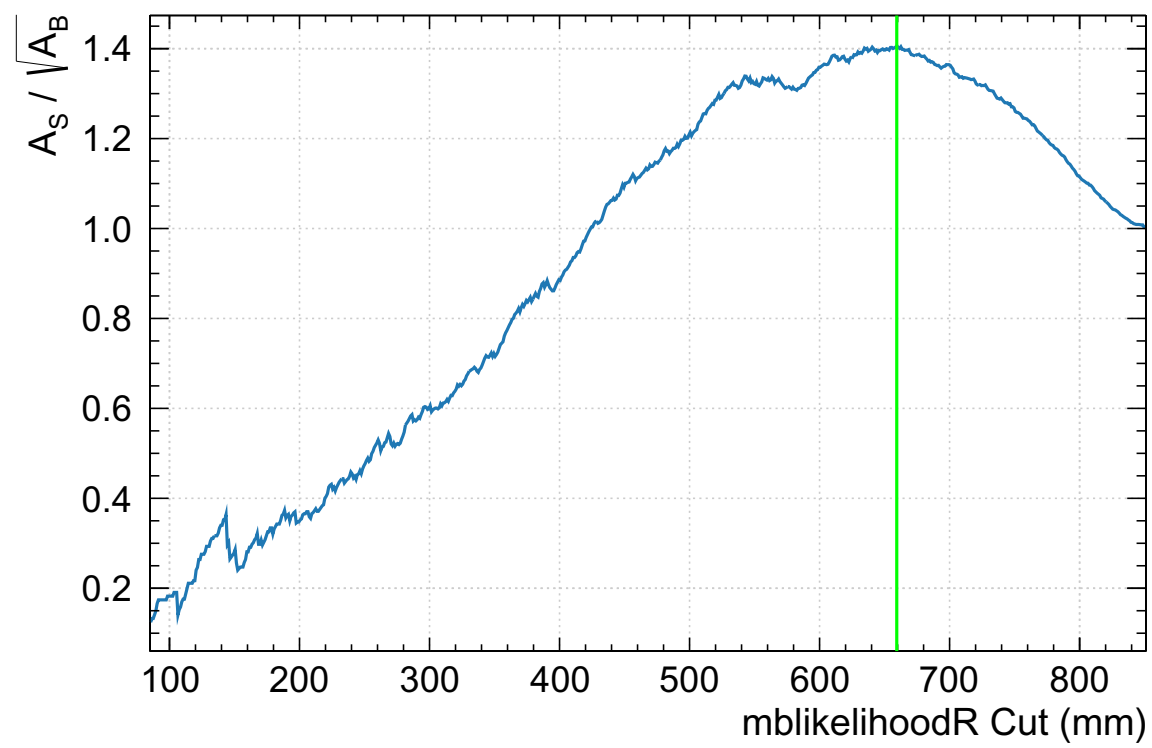

Figure 7.18: The $5.5 \mathrm{MeV} \gamma$ (Primakov) signal sensitivity as quantified by eq. 7.23 as a function of an upper radial cut for events with $41,000<\mathrm{qPE}(\mathrm{MC})<$ 42,000. The point where the sensitivity is maximized is labelled by the green line.

was found to be $660 \mathrm{~mm}$, yielding a signal acceptance of $79.4 \pm 0.7($ stat $) \%$ and a background acceptance of $32 \pm 1$ (stat)\%.

Since the calibration and axion search fits used non-optical MC, it was not possible to make the radial MC cut using "mblikelihoodR". Acceptance curves were drawn for both the reconstructed $\mathrm{MC}$ event position that was calculated using eq. 7.2 and the "mblikelihoodR" variable in the data. These are plotted in Figure 7.19, The data cut acceptance from the plot on the left is used to find the equivalent $\mathrm{MC}$ cut value in the plot on the right. The correspondence between the data and MC reconstructed positions is shown in Figure 7.20 .

\subsubsection{LY correction}

The DEAP-3600 detector undergoes temperature variations over time that can affect the qPE value reported by the detector. The light yield (LY) is here defined as the 

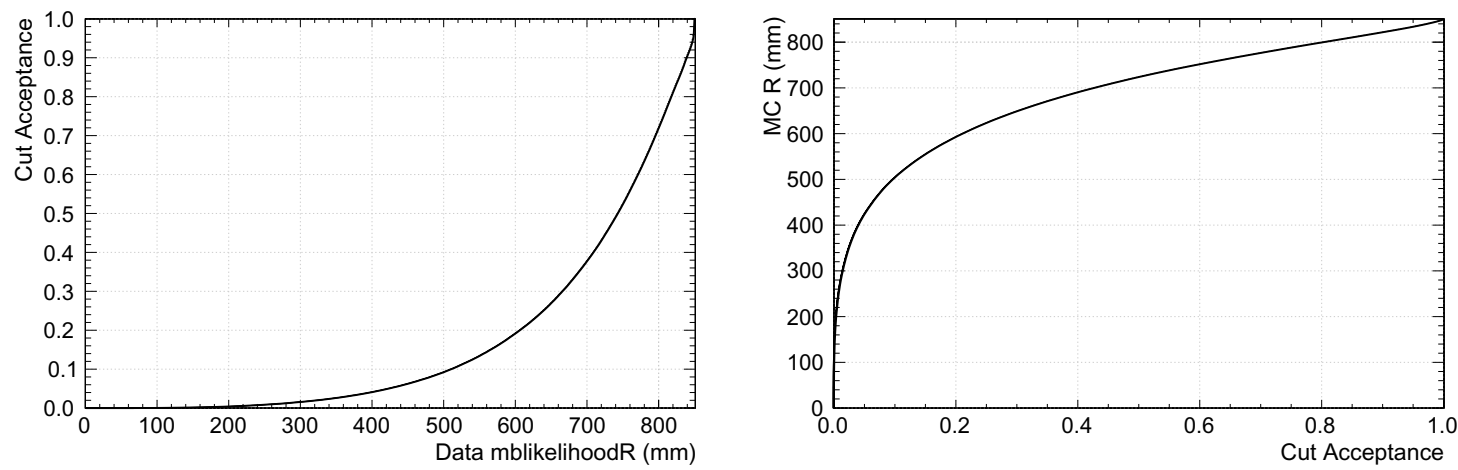

Figure 7.19: Left: The data radial cut acceptance vs the cut value. Right: The MC radial cut value vs the acceptance.

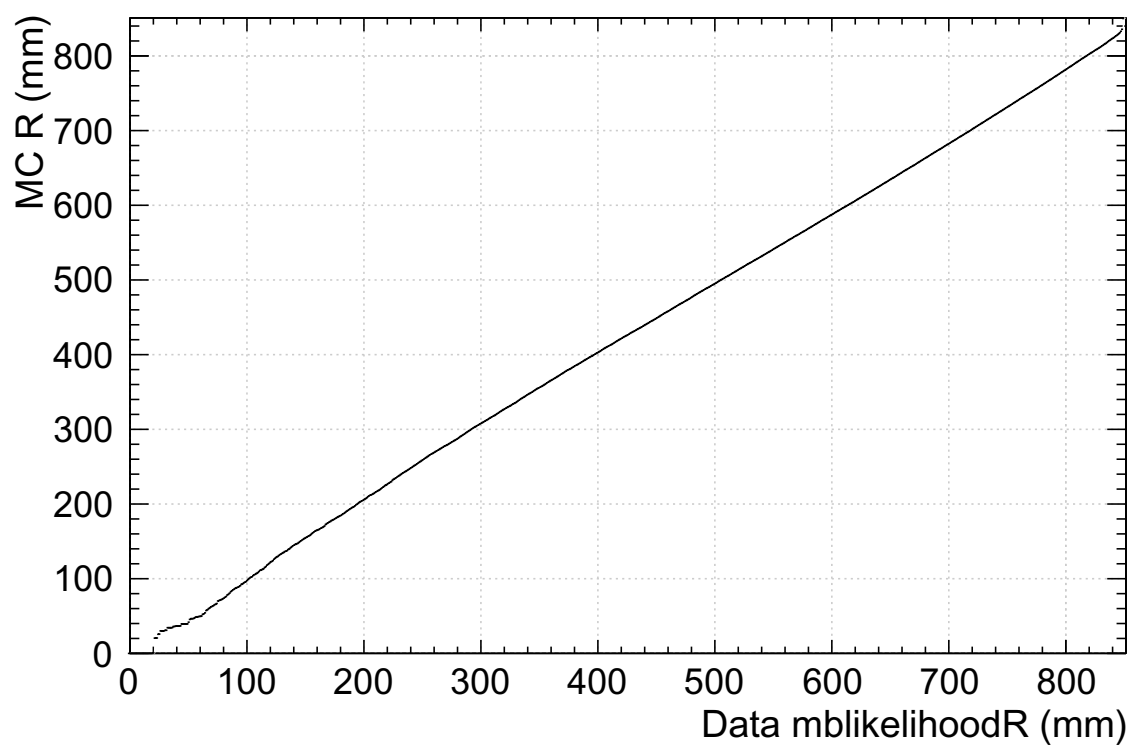

Figure 7.20: The MC radial cut value vs the data cut value as calculated using Figure 7.19 . 


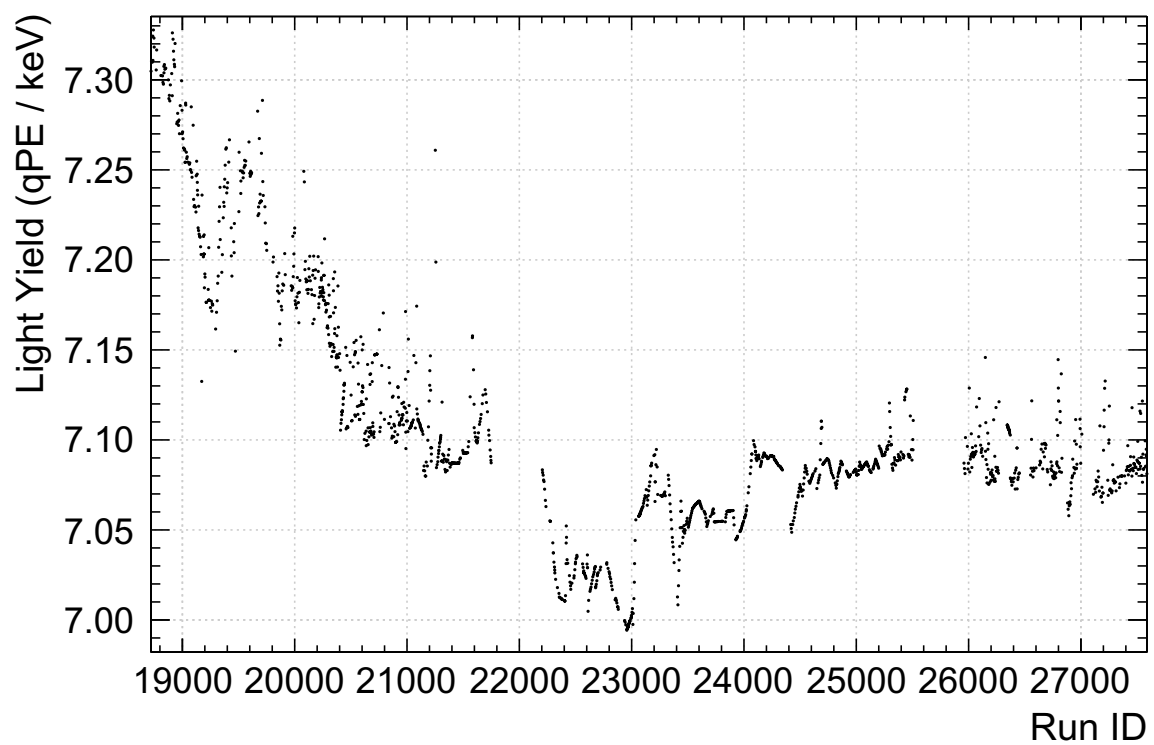

Figure 7.21: The DEAP-3600 detector light yield as a function of data run number. The measurements were made by a collaborator and this plot was compiled by the author. The first run was taken in November 2016, and the last run was taken in March 2020.

number of $\mathrm{qPE}$ observed per $\mathrm{keV}$ of energy deposited. In order to perform a fit on a dataset containing events from across a long period of time, a light yield correction was necessary. The LY can be measured for a sufficiently long physics data run by performing fits in qPE on known radioactive decay peaks. This was done by a collaborator for most of the non-calibration data runs which are used in the axion search. For the runs where the fits failed and for calibration runs, the LY was linearly interpolated (in units of time) based on the two runs that were closest in time. The resulting plot of LY vs data run number is shown in Figure 7.21. The effect of the light yield correction is demonstrated in Figure 7.22 .

\subsubsection{Background subtraction}

The AmBe source produces neutrons at a rate of $4.6 \pm 0.7 \mathrm{kHz}$. Though this is much higher than the rates of high energy $(>1.4 \mathrm{MeV})$ backgrounds in the detector, some 

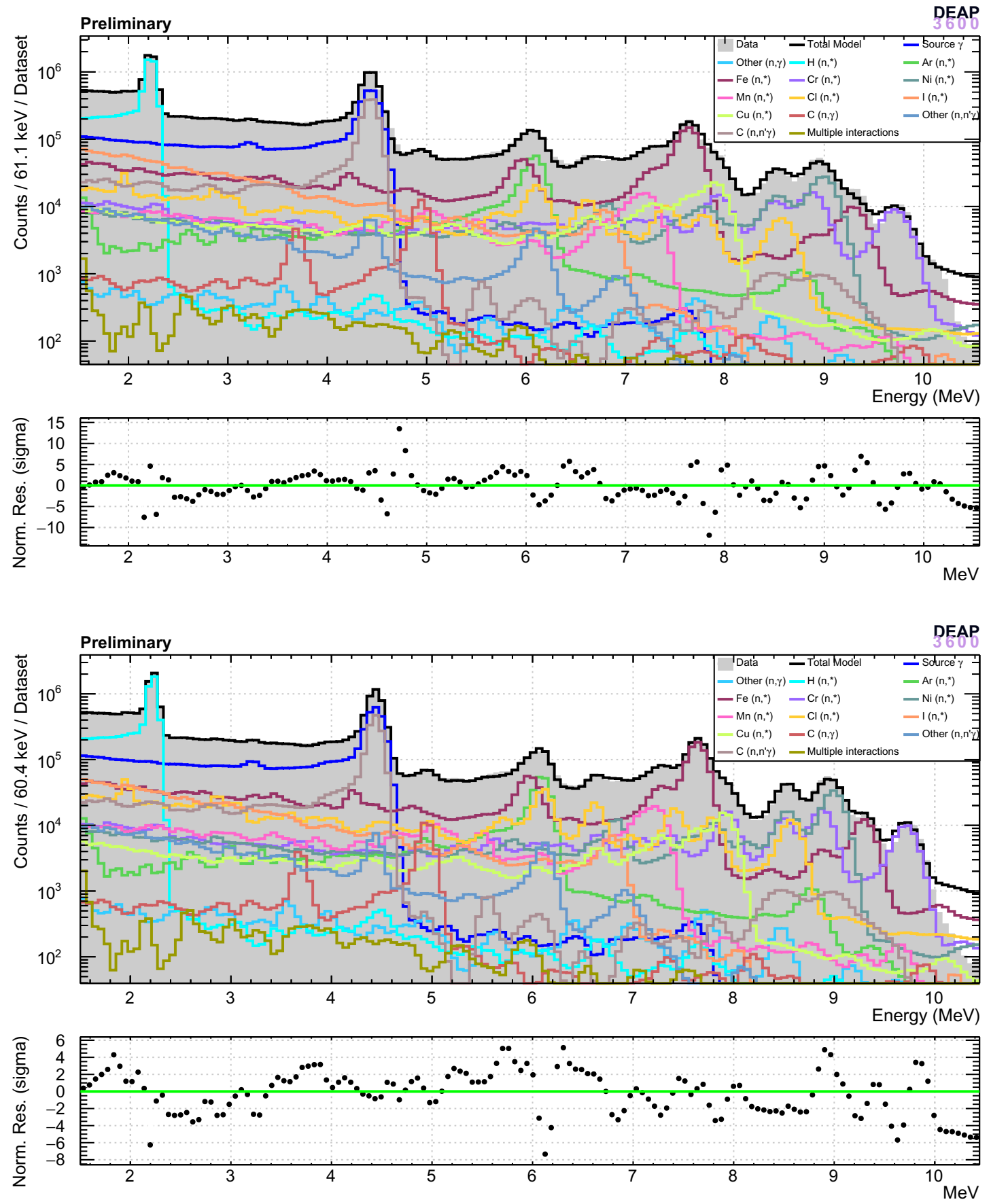

Figure 7.22: Fits to the full AmBe calibration dataset used in this work. Above: Fit without a light yield correction. Below: Fit with the light yield correction. Note that the residual Y-axis scale changes between plots. 


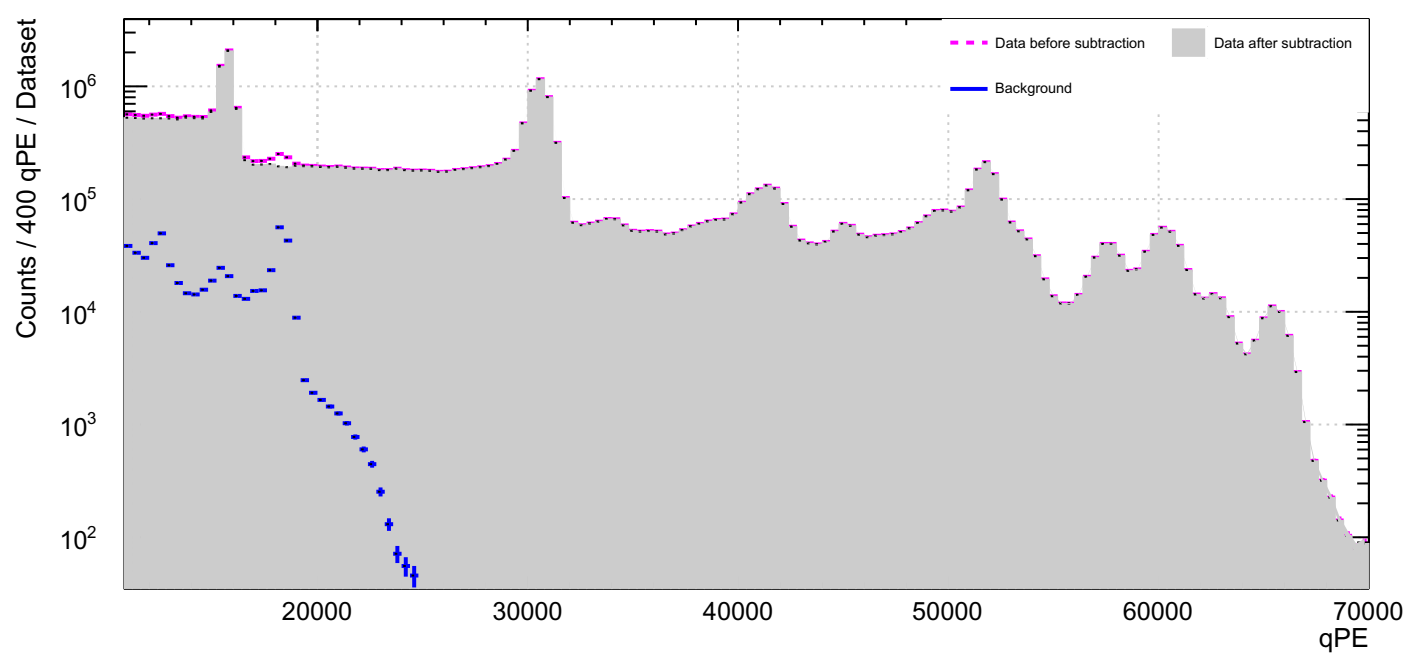

Figure 7.23: Histogram of the AmBe calibration dataset after radioactive detector background subtraction (shaded grey) overlaid with the background spectrum (blue) and the AmBe data prior to subtraction (dashed magenta).

radioactive decay peaks are still visible in the AmBe data. Rather than adding more components to the fit, this background was subtracted from the calibration dataset. For fits on single AmBe runs, the background spectrum was obtained by making a histogram of all the non-calibration physics data taken within a week before and after the calibration run and scaling it down to the live-time of the calibration run. This was generalized for fits on histograms containing events from several AmBe runs by assigning weights to physics runs according to the live-time of calibration runs that were taken within 1 week of the physics run. The background subtraction is shown in Figure 7.23 .

The background histogram typically covers a factor of 10 more live-time than the AmBe runs. The statistical uncertainty of the background histogram is thus negligible when compared to the statistical uncertainty on the AmBe data. The net effect is that the AmBe data in the bins where the background subtraction had a significant effect will have their statistical uncertainty slightly over-estimated, thus yielding a more conservative final result. 


\subsubsection{Saturation Correction}

As mentioned in Section 3.3, the total charge collected by the DAQ is lower for high energy events than would be expected if the charge were linearly proportional to the energy deposited in the detector. During the course of performing the analysis work required for this project, a colleague completed his work on an event processor that corrected both the PMT non-linearity and digitizer clipping effects in the data. My contribution to that work was as the primary reviewer of this processor. The effect on the fit is illustrated in Figure 7.24. This processor was run on all the calibration and physics data used in this work.

\subsection{Detector Datasets}

The two datasets involved in this work are the AmBe calibration dataset and the physics dataset. Both consist of several runs spread throughout the several year time frame of data collection.

\subsubsection{Making the AmBe Calibration Dataset}

The axion search fit was performed on data taken between November 2016 and March 2020. During this time there was the potential for the energy response of the detector to change due to temperature fluctuations or some other unknown causes. Since one of the primary goals of the AmBe calibration fits was to constrain the energy response function parameters for use in the axion search fit, it was necessary to have a calibration dataset that properly represented the energy response within the physics dataset.

The physics data was not taken at a constant rate. The fraction of physics data live-time per month is shown in Figure 7.25 . The AmBe calibration data taken 

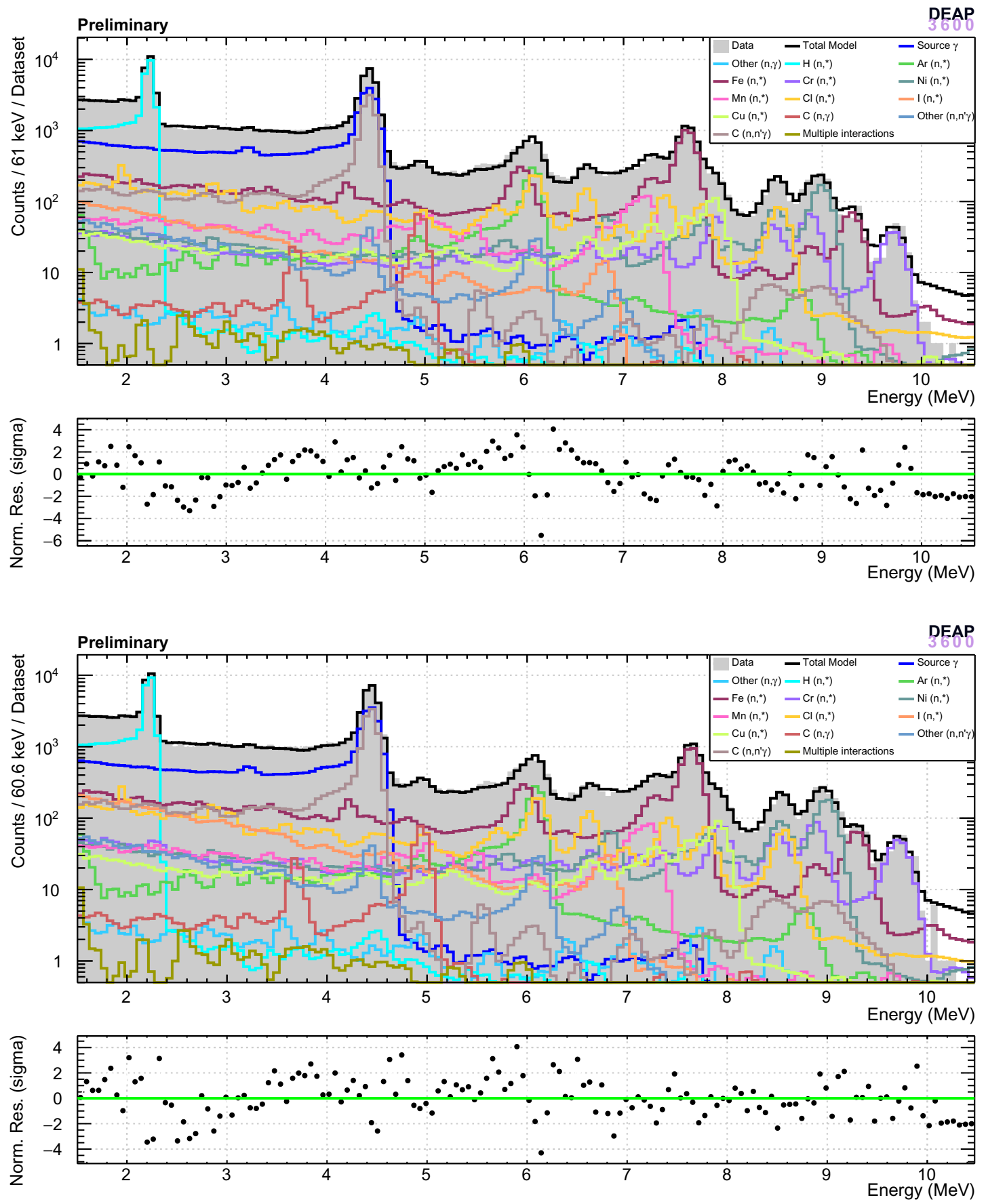

Figure 7.24: Fits to AmBe run 20714 without the saturation correction (above) and with the saturation correction (below). Note that the residual Y-axis scale changes between plots. 


\section{Physics Livetime per Month}

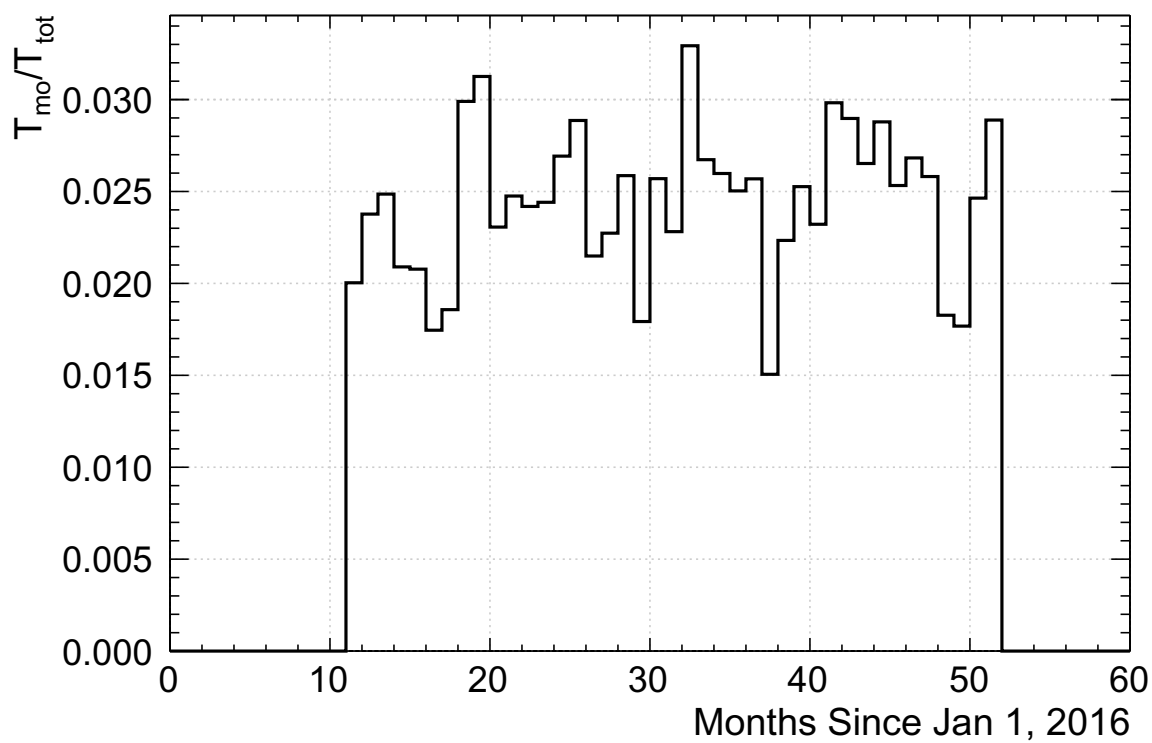

Figure 7.25: The fraction of the physics dataset live-time in 1 month wide bins.

per month is shown in Figure 7.26 (left). Note that runs are divided into several files called "sub-runs", which are approximately 2 to 5 minutes long. The number of AmBe sub-runs selected for the calibration dataset per month is shown in Figure 7.26 (right). These sub-runs were selected from the largest runs in a given month. For runs where not all sub-runs were needed, the sub-runs that were included in the calibration dataset were selected at random.

\subsection{Fit Validation with Toy Datasets}

Validation fits were performed using toy datasets. The purposes of these fits were to check whether the fitter displayed any bias when evaluating the parameters of interest, to measure the expected sensitivity, and to check that the fitter had proper coverage. 


\section{AmBe Subruns per Month}

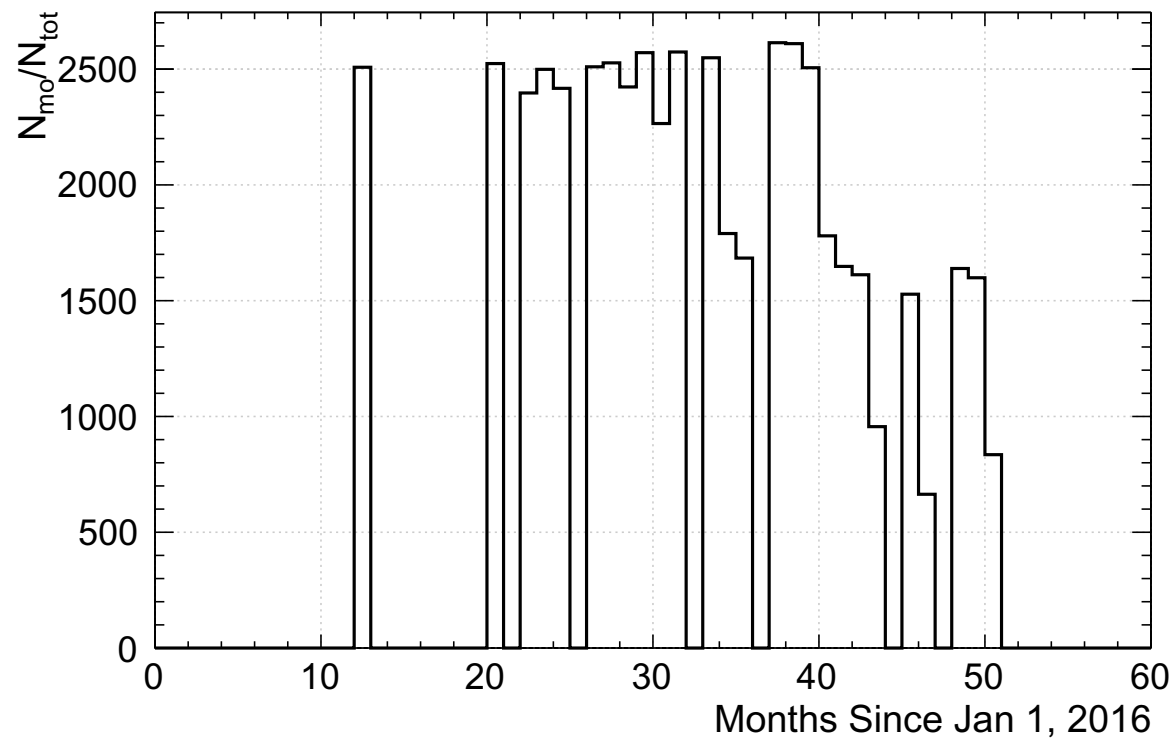

AmBe Subruns Used per Month

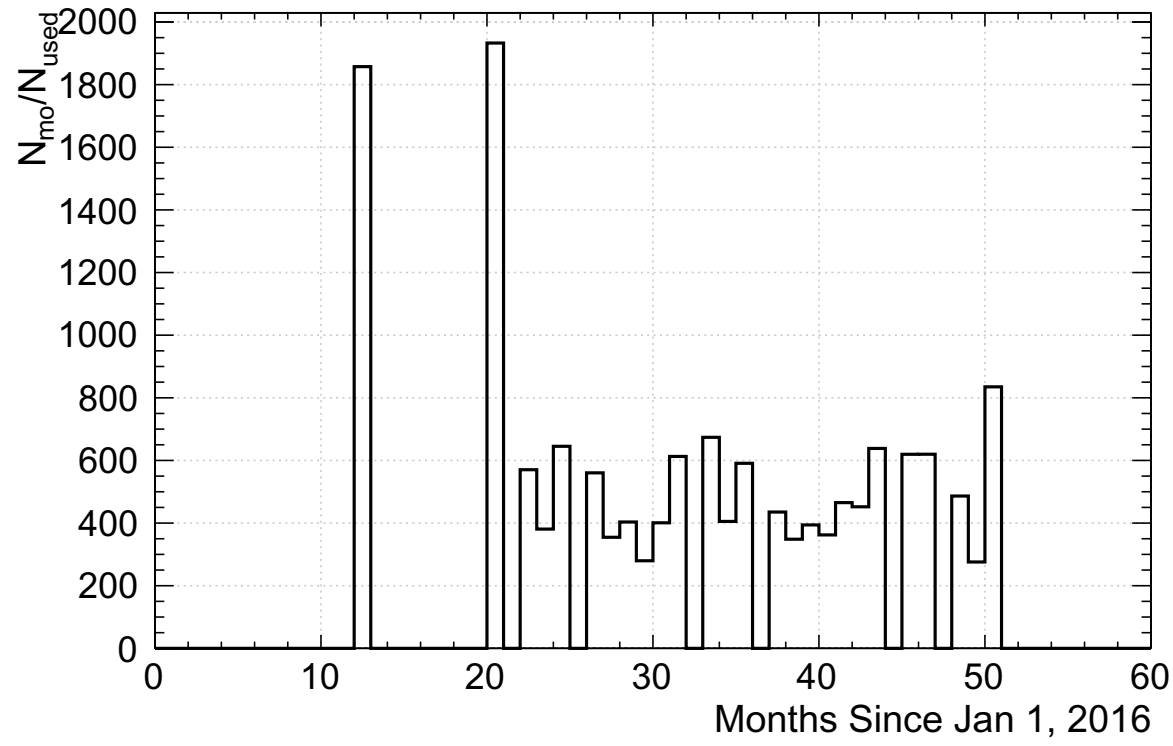

Figure 7.26: Left: Total number of AmBe sub-runs taken per month. Right: Number of AmBe sub-runs used in the AmBe calibration dataset for the axion search. 


\subsubsection{Toy Dataset Generation}

Toy datasets were generated from the MC model. The MC model was first calibrated on the AmBe data to set the response function parameters. The $\mathrm{MC}$ events that were generated in making the model were then read at random. The energy deposited by each event was inputted into the response function to get a mean PE value. A random number was then sampled from a Gaussian distribution with a mean given by the PE from the response function and a standard deviation given by the resolution component of the response function. This random number was used as the qPE value for the toy dataset event, and the weighted average of the energy depositions was used as the reconstructed event position.

The rates of the background and signal events were set based on previous fits to the electron recoil spectrum $[59]$ and, when applicable, the AmBe neutron production rate as quoted by the manufacturer and a mean rate of axion interactions as chosen when generating the dataset. For consistency, the axio-electric effect model (single $5.5 \mathrm{MeV}$ electron) was used to generate the axion signal for all tests. The differences between the output of the MC for the 4 axion models is quite small and mostly present in the tail, so it was decided that it was not worth quadrupling the already extensive CPU time required to perform the tests described below.

\subsubsection{Toy Physics Fits}

To validate the physics fit, toy physics datasets were generated and the model was fit to them with BAT. The axion signal event count followed a Poisson distribution. 20 datasets were generated each for Poisson means of 15 and 30 axion interactions, and 68 datasets were generated with 0 axion events (the number of datasets was limited by the MC statistics and available CPU time). The fitter was set to extract $68 \%$ confidence intervals from the marginalized axion normalization posterior using the 
method described in Section 7.5.3. If the fit has proper coverage, then the expectation is that $68 \%$ of the $68 \%$ confidence intervals will include the correct value. The fractions of the total fits where the correct axion count was included in the $68 \%$ confidence interval is shown in table 7.2 for three different axion signal strengths. From this table, it can be concluded that the fitter obtains acceptable coverage overall, with a possible over-coverage in the 0 axion case. This over-coverage implies that a slight overestimate of the uncertainty is expected when using this fitter, which will result in a slight reduction in the sensitivity. Plots of some sample fits are shown in Figures 7.27 . 7.29. Histograms of the axion fit parameter value and its uncertainty are shown for each of the three cases in Figure 7.30 .

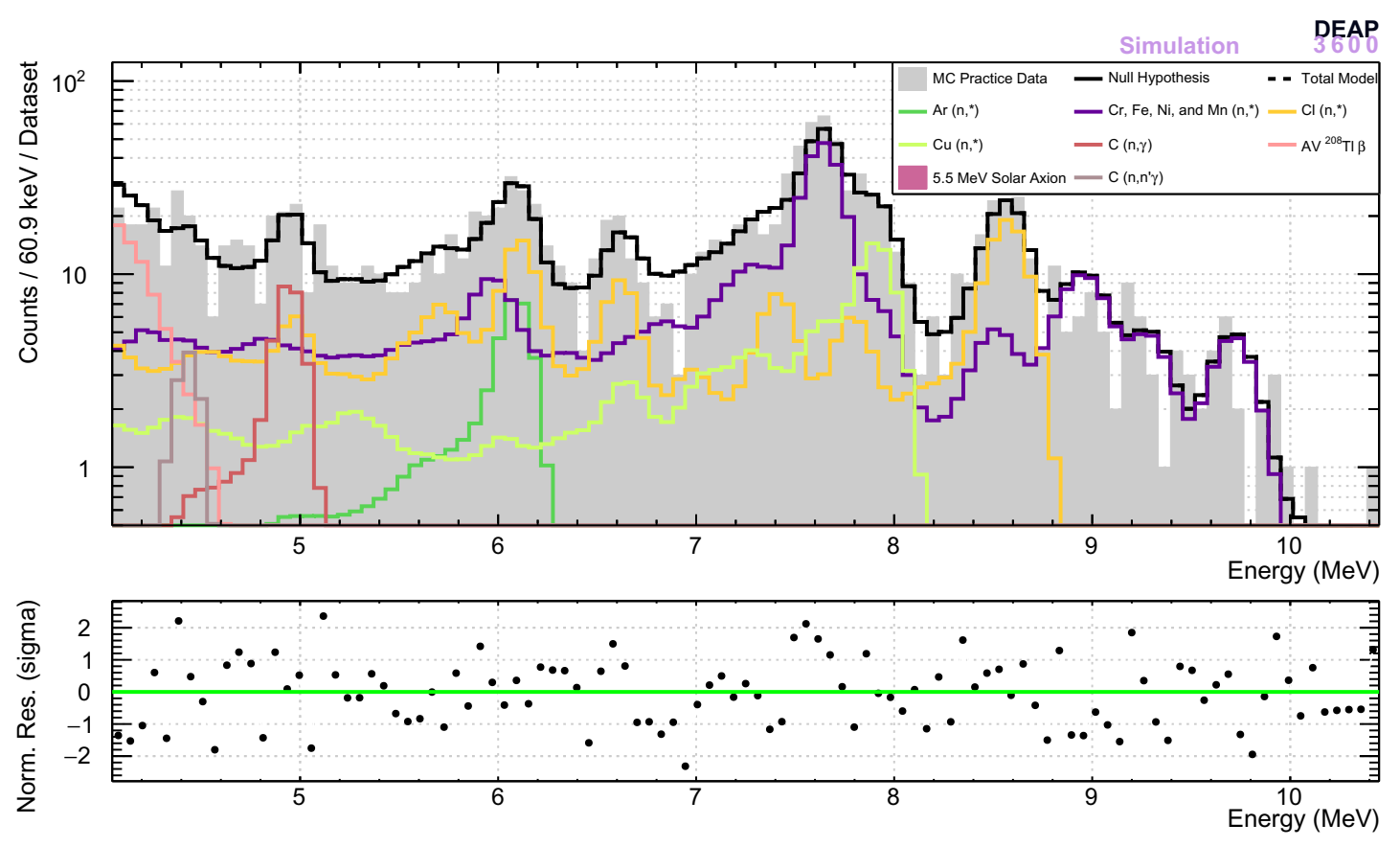

Figure 7.27: Sample toy physics data fit, selected at random, with $<\mathrm{N}>=0$. 

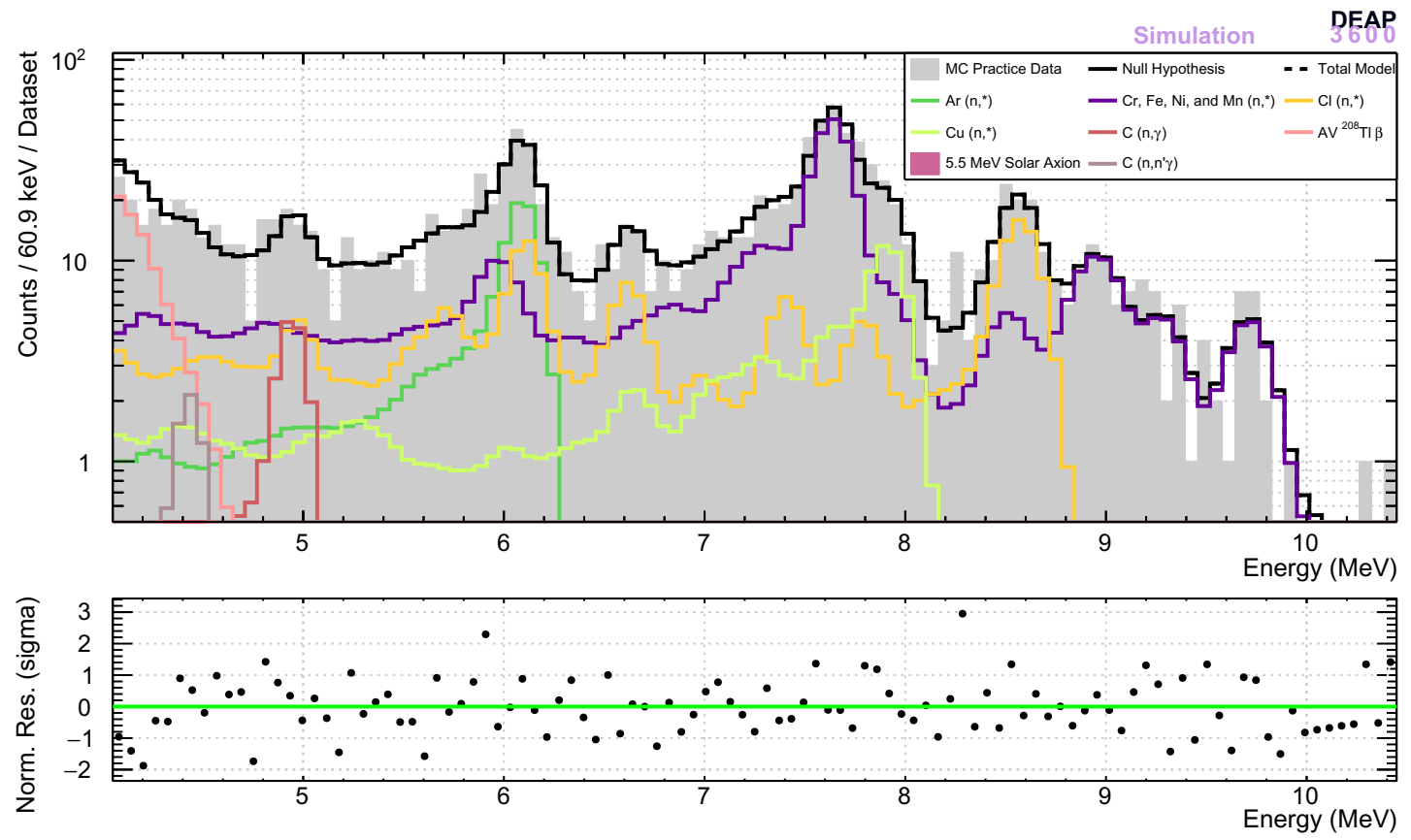

Figure 7.28: Sample toy physics data fit, selected at random, with $<\mathrm{N}>=15$.

\subsubsection{Toy AmBe Fits}

37 toy AmBe datasets, each with three hours of simulated live-time, were generated, and the fit run on them using BAT. As in the case with the physics fits, the number of datasets was limited by the MC statistics and available CPU time. The parameters of interest for this fit are the 5 response function parameters, so these were the focus of the coverage tests. As in the previous section, the $68 \%$ confidence intervals were set according to the method described in Section 7.5.3. If the fit coverage is good, we would expect $68 \%$ of the resulting $68 \%$ confidence intervals to include the correct value for a given fit parameter. The fractions of confidence intervals that include the correct parameter value are given in table 7.3 for the 5 response function parameters, and histograms of the parameter values and their uncertainties are in Figures 7.31 and 7.32. Sample fits are shown in Figure 7.33. From these tests it can be concluded that the fitter does not display over significant biasing and contains proper coverage 

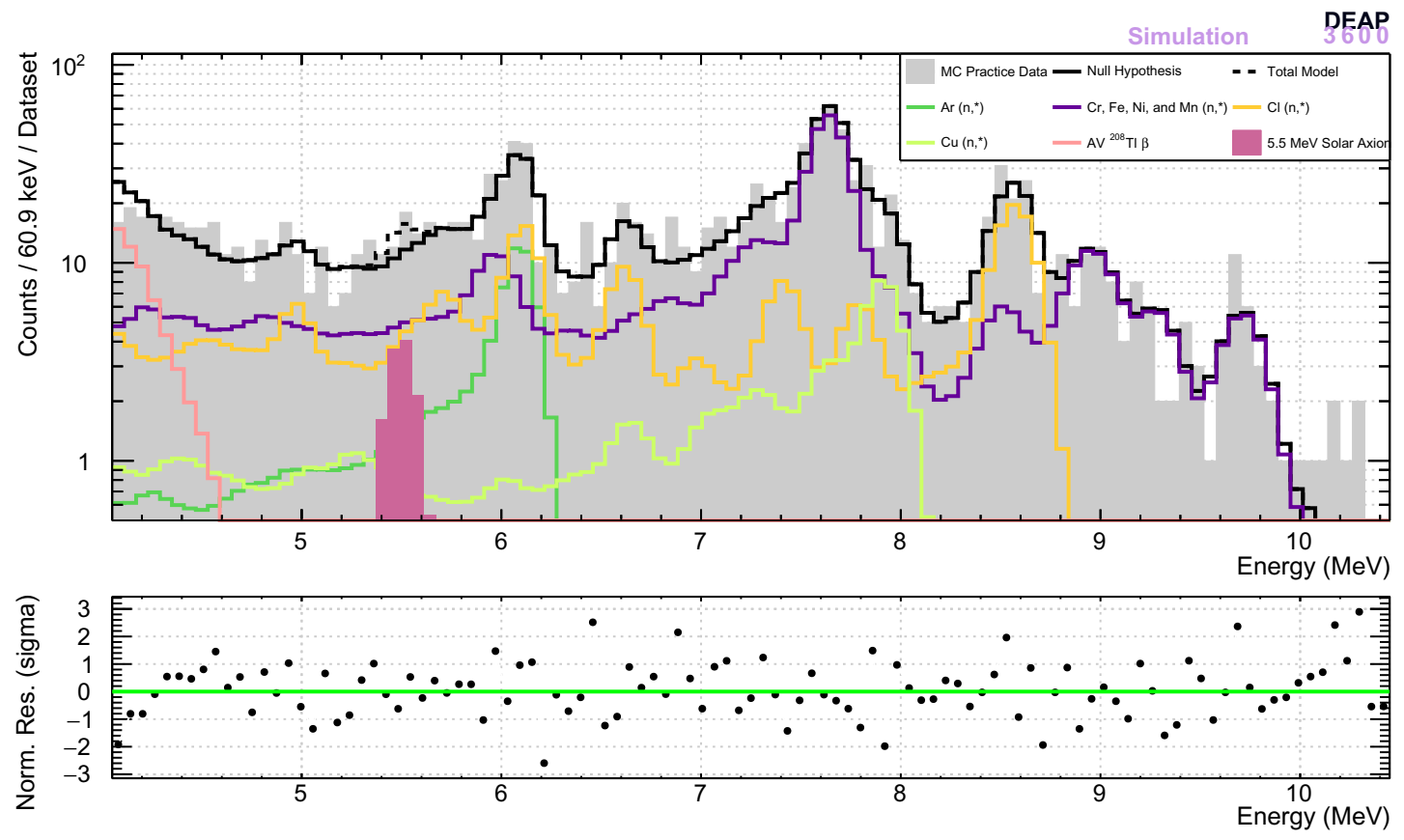

Figure 7.29: Sample toy physics data fit, selected at random, with $<\mathrm{N}>=30$.

of the response function parameter values.

\subsubsection{Sensitivity Studies}

After fitting the model to several toy datasets, the axion limit was evaluated by integrating from 0 events up to the point where $90 \%$ of the total marginalized posterior integral had been included. The point where the integral ended was taken as the upper axion event limit. This was repeated for all toy physics dataset fits, and the result was averaged. The average $90 \%$ upper limit is given as the first row in table 7.4 . This gives an estimate on the expected $90 \%$ upper limit that could be obtained from this fit for the three different axion signal strengths tested.

If the method in Section 7.5.3 is applied (integrating the marginalized posterior starting at the largest bin and adding the next largest bins on the left or the right), the resulting confidence interval will not necessarily include 0 axion events. In this case, 
an "excess" of events would be reported. If we have proper coverage, the probability of including the correct result will be equal to the confidence level. For instance, in the case where the axion expectation is 0 , we would expect $68 \%$ of the $68 \%$ confidence intervals to include 0 . Thus the probability of an excess should be $32 \%$. Similarly, a $90 \%$ confidence interval should give a $10 \%$ probability of excess and a $95 \%$ confidence interval should give a $5 \%$ probability of excess. Table 7.4 gives the resulting probabilities of excess for the three different confidence levels and for three different axion event expectations. Since the numbers in the second, third, and fourth rows of the first column all contain 22\%,10\%, and 5\% respectively, it can be concluded that the fitter is yielding proper coverage of the axion signal normalization.

The subsequent rows list the probability that an excess will be observed rather than a limit, using the method described in Section 7.5.3. The distributions of the 90\% CL upper limits are shown in Figure 7.34.

\subsection{Final Blinded Fits}

\subsubsection{Final AmBe Fit}

The final AmBe calibration fit to the full AmBe dataset, after applying all corrections, is shown in Figure 7.35. The parameter values are given in table 7.5. The marginalized posterior distributions are shown in Figure 7.36, and the correlation matrix is in Figure C.1.

\subsubsection{Final Blinded Physics Fit}

The marginalized posteriors for the energy response function parameters from the AmBe fit (first 5 plots in Figure 7.36) were used as priors in the physics fit, strongly constraining those parameters. The physics fit, with the axion region of interest 
blinded, is shown in Figure 7.37. The parameter values are in table 7.7, and the posteriors and correlation matrix (dominant correlations) are in Figure 7.38 and table 7.8 respectively.

The neutron capture $\gamma$ fit parameter normalizations in table 7.7 would be approximately equal to 1 if the GEANT4 model matched the data well. As discussed earlier, the GEANT4 model has issues that prevent it from properly representing the data. However, the Mn normalization seems unrealistically high, especially when compared to the normalizations of the other neutron capture $\gamma$ fit parameters. This may be a result of the fitter attempting to "fill" an excess caused by an un-modelled background. The fit was performed again with the Mn component fixed to twice the GEANT4 prediction. The resulting fit is given in Figure 7.39, and the parameter values are in table 7.9

\subsubsection{Effect of Systematic Uncertainties}

All systematic uncertainties that could be easily integrated into the BAT fit were included in the final result. To get a sense of the effect of these uncertainties on the final result, a series of fits were run with at most one nuisance parameter free to float. The one free nuisance parameter was thus the only systematic uncertainty included in the marginalized axion event count posterior. These fits were run on a single toy dataset, which was derived from the best physics fit by sampling from the fitted model. To reduce the effect of statistical uncertainties, the statistics in this toy dataset were set to be a factor of 10 greater than in the real physics data. The resulting axion event limits on these toy fits were thus larger by a factor of approximately $\sqrt{10}$ than what would be expected based on the previously mentioned sensitivity studies. The chart showing the fitted axion limits is in Figure 7.40. This chart was made by fixing all parameters during the fit except the axion normalization and the parameter listed on the Y-axis. Also shown are the limits with the MC bin error excluded and with 
the detector mass uncertainty included. The larger the limit, the greater the effect of the parameter on the final result.

\subsection{Exclusion Curves}

\subsubsection{Projected Exclusion Curves Prior to Unblinding}

The current world-leading limits in the search for $5.5 \mathrm{MeV}$ solar axions come from Borexino [49]. Due to the substantially smaller size of DEAP-3600, it is not possible to surpass Borexino's limits.

Since four processes and two models are under consideration, a total of eight limits can be computed. The exclusion limit plots shown here give the projected sensitivity of this search (90\% confidence) on a dataset with a live-time of 802 days. These plots are shown in Figure 7.41, and give the limits for $g_{A e}$ in the hadron model, $\left|g A e \times g_{3 A N}\right|$ in the GUT model, and $g_{A \gamma}$ in the hadronic model.

\section{Limits involving $g_{A e}$}

The (Poisson) mean numbers of events due to Compton conversion and the axioelectric effect in DEAP-3600 are given by:

$$
\begin{aligned}
& S_{C C}=\phi_{A} \sigma_{C C} N_{A r} T \\
& S_{A E}=\phi_{A} \sigma_{A E} N_{A r} T
\end{aligned}
$$

where $\phi_{A}$ is given by eq. 2.19, $\sigma_{C C}$ is given by eq. 2.13, $\sigma_{A E}$ is given by eq. 2.15, and $N_{A r}=(4.94 \pm 0.14) \times 10^{28}$ is the number of argon atoms in the detector. Since $\sigma_{C C, A E} \propto g_{A e}^{2}$ and $\phi_{A} \propto\left(g_{3 A N}\right)^{2}$, the condition that $S_{C C}<S_{C C}^{\lim }$ and $S_{A E}<S_{A E}^{\lim }$ can be used to draw the curves in Figure 7.41 (middle), showing the projected limit on $\left|g A e \times g_{3 A N}\right|$. The hadronic (KSVZ) axion model relates the axion-nucleon coupling to 
the axion mass through eq. 2.7. The exclusion curves for models with this relation are given in Figure 7.41 (top). These exclusion curves are only valid under the assumption that the axions do not decay in transit from the sun.

\section{Limits involving $g_{A \gamma}$}

The mean numbers of events due to inverse Primakov conversion and axion decay to $2 \gamma$ are given by:

$$
\begin{aligned}
S_{P C} & =\phi_{A} \sigma_{P C} N_{A r} T \\
S_{2 \gamma} & =N_{\gamma} T,
\end{aligned}
$$

where $\sigma_{P C}$ is given by eq. 2.14 and $N_{\gamma}$ is given by eq. 2.22 . Using the LAr mass and assuming a LAr density of $1.40 \mathrm{~g} / \mathrm{cm}^{3}$, the DEAP-3600 LAr volume is $(2.34 \pm 0.07) \times$ $10^{6} \mathrm{~cm}^{3}$. Since the reduction in axion flux due to decays to $2 \gamma$ in transit from the sun must be taken into account, there is no direct proportionality between the axion coupling constants and the expected numbers of events. However, in the hadronic axion model, the constraint on $g_{3 A N}$ (eq. 2.7) makes it possible to draw an exclusion curve, which is shown in Figure 7.41 (bottom).

\subsection{Unblinding and Excess in Physics Side-band}

As can be seen in Figure 7.37, an unexpected excess above the background model was observed at $6.4 \mathrm{MeV}$. At the time of writing, this excess remains unexplained. Different materials that are present in the real detector but absent from the Monte Carlo may be a potential source of neutron capture $\gamma$ lines. However, none of the materials so far tried have yielded $\gamma$ lines at 6.4 MeV. Data processing effects, muons, $\beta$ decays, $\alpha$ capture reactions, and other physics processes have been, and are being, explored as potential sources of additional $\gamma$ lines. 
If the source cannot be identified in a reasonable time scale, a limit-setting search will be done, excluding the possibility that a statistical excess will be reported in the axion region of interest. 


\begin{tabular}{|c|c|}
\hline Component & Description \\
\hline Const. E Scale & $C$ in eq. 7.3 \\
\hline Linear E Scale & $B$ in eq. 7.3 \\
\hline Quad. E Scale & $A$ in eq. 7.3 \\
\hline Linear Res. & $r_{2}$ in eq. 7.4 \\
\hline Quadratic Res. & $r_{1}$ in eq. 7.4 \\
\hline Source $\gamma$ & $\gamma$ lines produced within the AmBe source \\
\hline $\mathrm{X}\left(\mathrm{n},{ }^{*}\right)$ & Events triggered primarily by capture or scatter on element $\mathrm{X}$ \\
\hline $\mathrm{C}(\mathrm{n}, \gamma)$ & Events triggered primarily by capture on carbon \\
\hline $\mathrm{C}(\mathrm{n}, \mathrm{n} \gamma)$ & Events triggered primarily by inelastic scatter on carbon \\
\hline Other $(\mathrm{n}, \gamma)$ & Neutron capture spectra that fitter is not sensitive to \\
\hline Other $\left(\mathrm{n}, \mathrm{n}^{\prime} \gamma\right)$ & Low rate neutron inelastic scatters that are not included above \\
\hline Other (fixed) & Other $\gamma$ lines produced by processes other than neutron capture \\
\hline Multiple interactions & Events where two captures or two scatters occur on different elements \\
\hline $4.7 \mathrm{MeV}{ }^{40} \mathrm{Ar} \gamma$ & Intensity of $4.7 \mathrm{MeV}{ }^{40} \mathrm{Ar} \gamma$ line \\
\hline 7.6 $\mathrm{MeV}^{56} \mathrm{Fe} \gamma$ & Intensity of $7.6 \mathrm{MeV}{ }^{56} \mathrm{Fe} \gamma$ line \\
\hline $\mathrm{AV}^{208} \mathrm{Tl} \beta$ & $\beta$ spectrum from ${ }^{208} \mathrm{Tl}$ decays near the $\mathrm{AV}$ inner surface \\
\hline LAr ${ }^{208} \mathrm{Tl} \beta$ & $\beta$ spectrum from ${ }^{208} \mathrm{Tl}$ decays within the $\mathrm{LAr}$ \\
\hline
\end{tabular}

Table 7.1: A description of the components that were allowed to float in final calibration and physics fits. Not all components were floated in all fits. Note that captures take precedence over inelastic scatters when assigning to a spectrum events in which multiple interactions occur in the same $13.5 \mu$ s event window. Also note that the MC statistical uncertainty is also included in the likelihood function, and thus is factored into the marginalized posteriors.

\begin{tabular}{|c|c|c|}
\hline Axion Signal $<\mathbf{N}>$ & Coverage $(\mathbf{6 8 \%}$ uncert.) & Coverage (68\% CI) \\
\hline$<\mathbf{N}>=\mathbf{0}$ & $0.794_{-0.061}^{+0.051}$ & {$[0.73,0.85]$} \\
$<\mathbf{N}>=\mathbf{1 5}$ & $0.70_{-0.14}^{+0.11}$ & {$[0.56,0.81]$} \\
$<\mathbf{N}>=\mathbf{3 0}$ & $0.60_{-0.14}^{+0.12}$ & {$[0.46,0.72]$} \\
\hline
\end{tabular}

Table 7.2: The fraction of the toy data fits that included the correct mean number of axions for $\langle\mathrm{N}\rangle=0,15$, and 30 axions. The centre column gives this fraction with statistical uncertainties (limited by the number of fake datasets used), while the right column gives the same information but formatted as a confidence interval based. If the fits have proper coverage, these fractions should be consistent with 0.68 . 

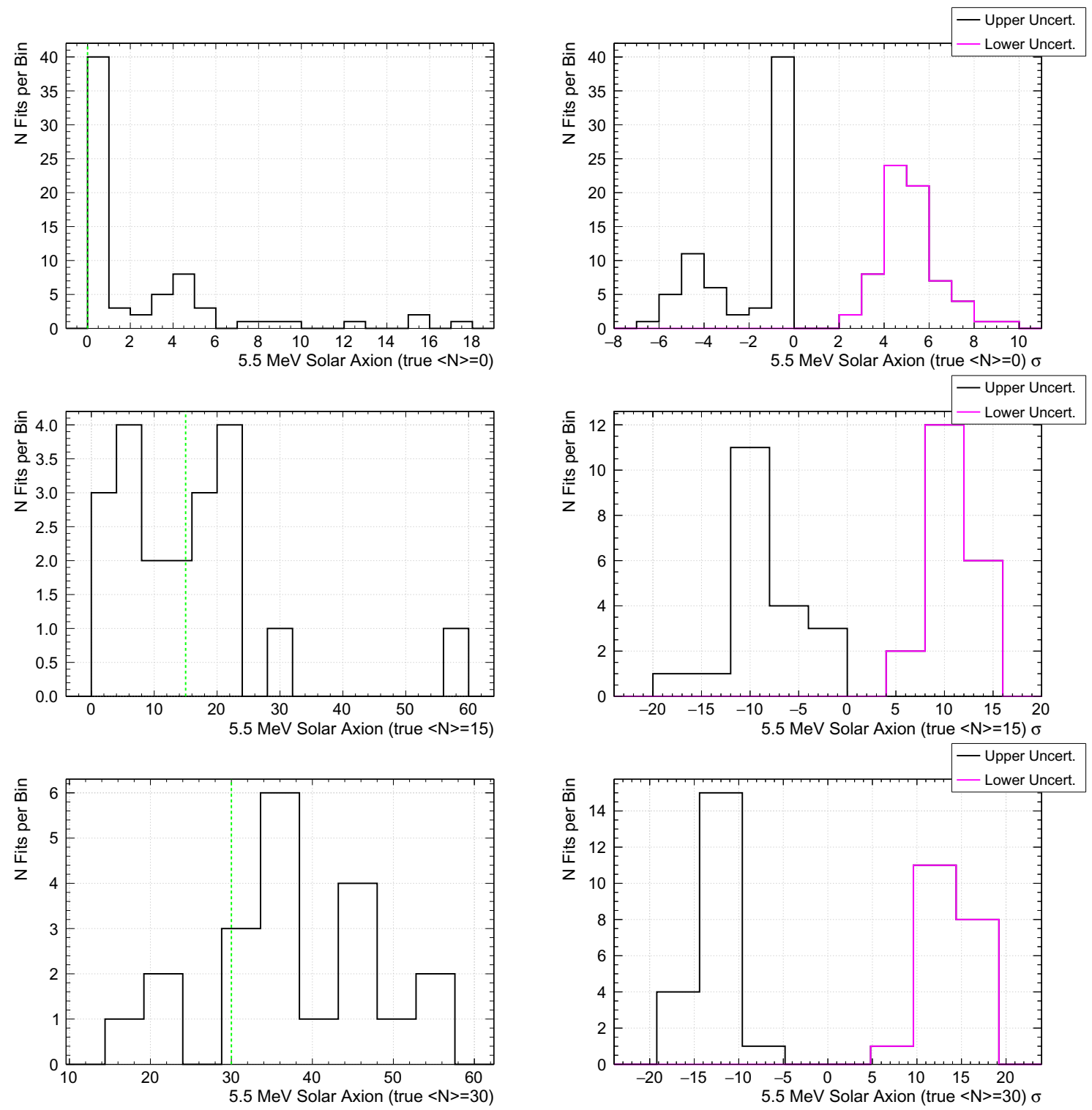

Figure 7.30: Distributions of fitted axion event counts (left) and their lower and upper uncertainties (right) from toy physics data fits. The true value is labeled with a dashed green line. In the plots on the right, the histograms of the lower error bars are black, while the histograms of the upper error bars are magenta. 


\begin{tabular}{|c|c|c|}
\hline Parameter & Coverage (68\% uncert.) & Coverage (68\% CI) \\
\hline Const. E Scale & $0.811_{-0.087}^{+0.067}$ & {$[0.72,0.88]$} \\
Linear E Scale & $0.784_{-0.089}^{+0.071}$ & {$[0.69,0.85]$} \\
Quad. E Scale & $0.784_{-0.089}^{+0.071}$ & {$[0.69,0.85]$} \\
Linear Res. & $0.730_{-0.093}^{+0.078}$ & {$[0.64,0.81]$} \\
Quadratic Res. & $0.676_{-0.095}^{+0.084}$ & {$[0.58,0.76]$} \\
\hline
\end{tabular}

Table 7.3: The fraction of $68 \%$ confidence intervals that include the correct parameter value for the 5 energy response function parameters after performing the toy AmBe data fits. With proper coverage, $68 \%$ of these intervals should include the correct parameter value.

\begin{tabular}{|l|c|c|c|}
\hline & $\langle\mathbf{N}>=\mathbf{0}$ & $\langle\mathbf{N}>=\mathbf{1 5}$ & $\langle\mathbf{N}>=\mathbf{3 0}$ \\
\hline Average 90\% upper limit & $25 \pm 11$ axions & $33 \pm 13$ axions & $57 \pm 11$ axions \\
Prob. of excess (68\% CL) & $21_{-05}^{+06 \%}$ & $70_{-14}^{+11} \%$ & $100_{-9}^{+0} \%$ \\
Prob. of excess (90\% CL) & $7_{-3}^{+4} \%$ & $40_{-12}^{+14} \%$ & $95_{-11}^{+4} \%$ \\
Prob. of excess (95\% CL) & $7_{-3}^{+4} \%$ & $30_{-11}^{+14} \%$ & $95_{-11}^{+4} \%$ \\
\hline
\end{tabular}

Table 7.4: Sensitivity of axion search for different axion signal strength $\langle\mathrm{N}\rangle$, calculated by performing fit to toy datasets. First row: The average $90 \%$ upper limit obtained from the fits to fake datasets containing an axion expectation of $<\mathrm{N}>$ events. Second to third rows: The probably of the given CL not including 0 axion events for various $\langle\mathrm{N}\rangle$. Since the final result would exclude 0 up to the specified CL, the result would be reported as an excess. 

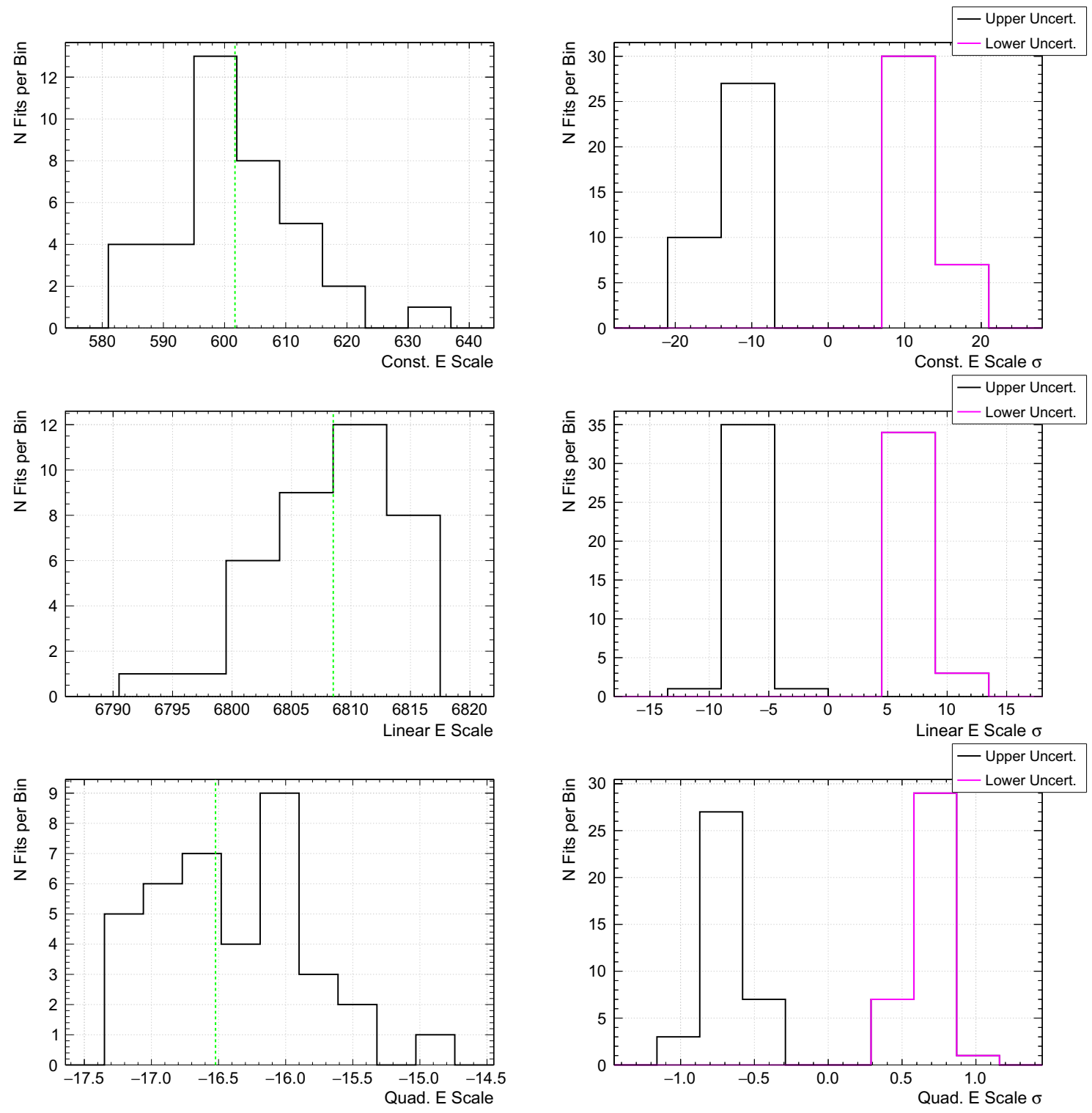

Figure 7.31: Distributions of response function energy scale parameter values (left) and their lower and upper uncertainties (right) from toy AmBe data fits. The true value is labeled with a dashed green line. In the plots on the right, the histograms of the lower error bars are black, while the histograms of the upper error bars are magenta. 

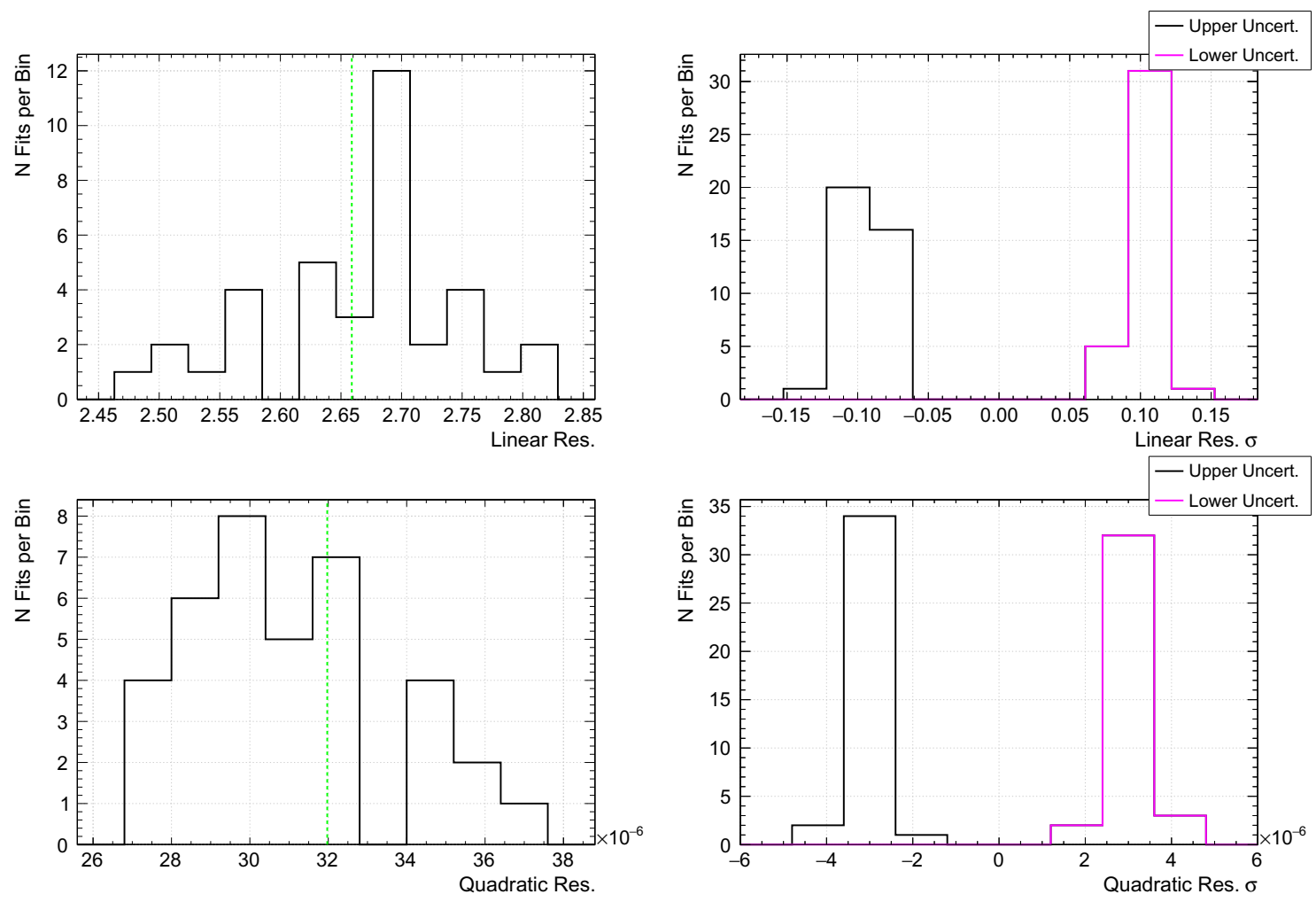

Figure 7.32: Distributions of response function resolution parameter values (left) and their lower and upper uncertainties (right) from toy AmBe data fits. The true value is labeled with a dashed green line. In the plots on the right, the histograms of the lower error bars are black, while the histograms of the upper error bars are magenta. 

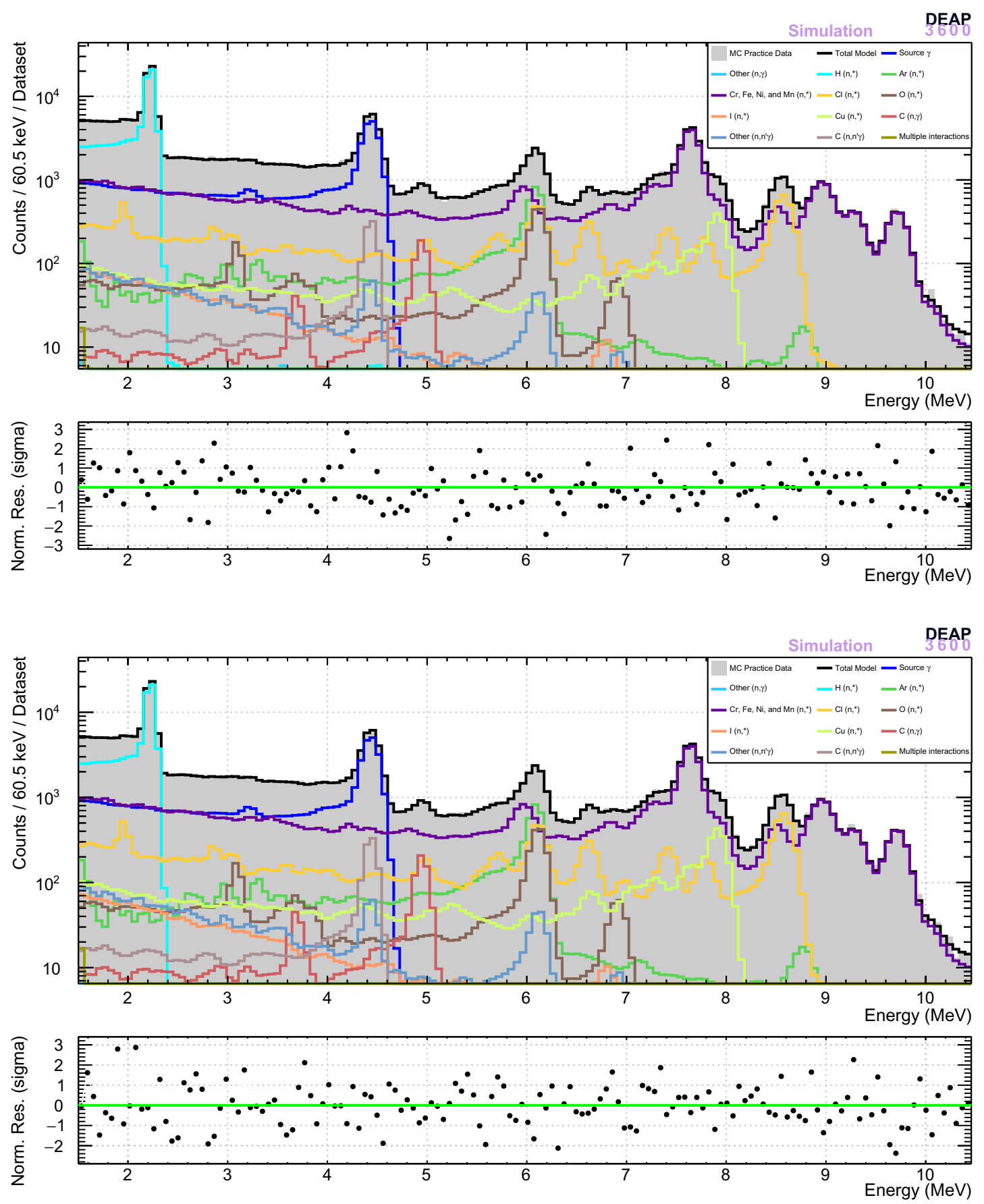

Figure 7.33: Samples of toy AmBe data fits, selected at random. 


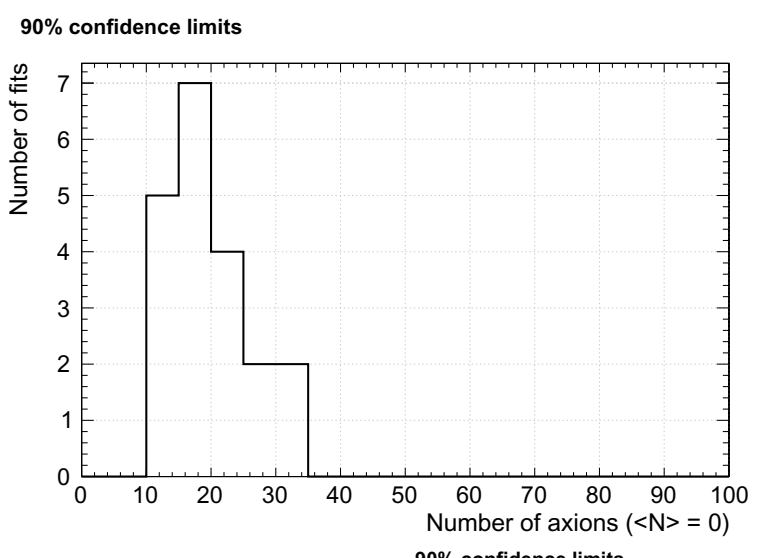

$90 \%$ confidence limits

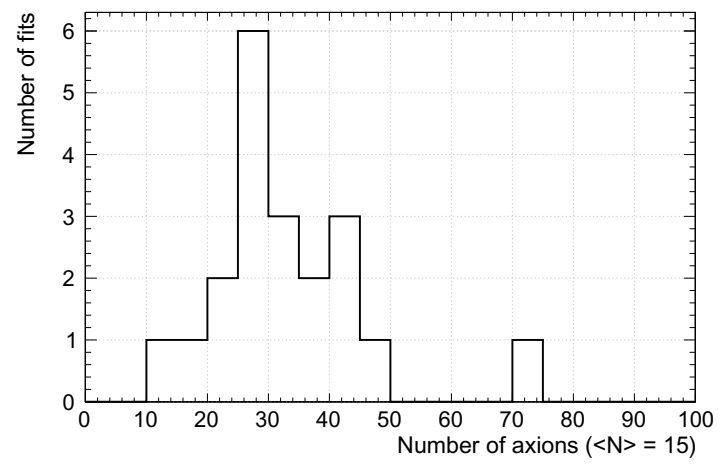

$90 \%$ confidence limits

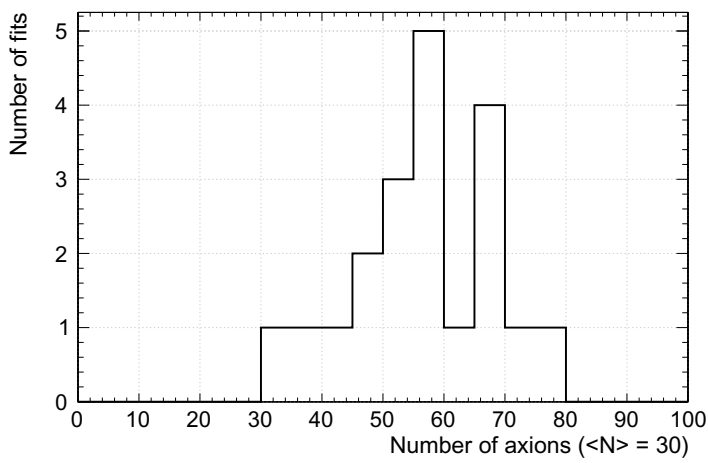

Figure 7.34: The distributions of $90 \%$ CL upper limits derived from toy dataset fits with expected axion signals $<\mathrm{N}>$ of 0,15 , and 30 . 


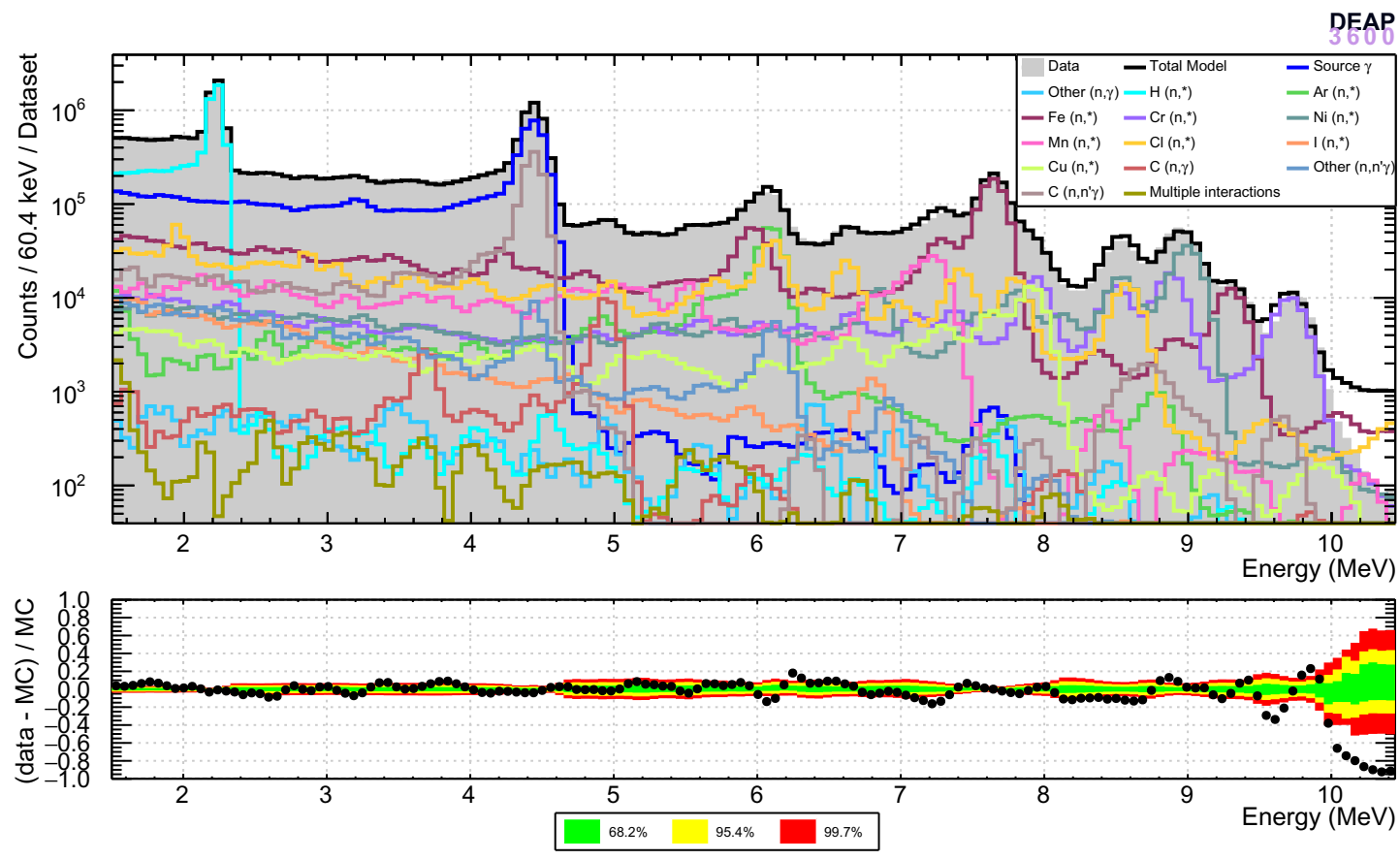

Figure 7.35: The complete AmBe BAT fit that provided the response function priors for the physics fit discussed in Section 7.10.2. The lower plot includes statistical and systematic uncertainties. 


\begin{tabular}{|c|c|c|}
\hline Parameter & Best fit value & $68 \%$ credibility interval \\
\hline Const. E Scale & $548.0_{-4.1}^{+3.0}$ & {$[543.9,551.1]$} \\
\hline Linear E Scale & $6853.7_{-1.7}^{+1.7}$ & {$[6852.0,6855.4]$} \\
\hline Quad. E Scale & $-19.51_{-0.16}^{+0.13}$ & {$[-19.67,-19.38]$} \\
\hline Linear Res. & $2.972_{-0.081}^{+0.076}$ & {$[2.89,3.05]$} \\
\hline Quadratic Res. & $0.0000300_{-0.0000022}^{+0.0000023}$ & {$[0.0000278,0.0000323]$} \\
\hline Source $\gamma$ & $1.277_{-0.027}^{+0.024}$ & {$[1.250,1.301]$} \\
\hline Other $(\mathbf{n}, \gamma)$ & 1 & (fixed) \\
\hline $\mathbf{H}(\mathbf{n}, *)$ & $0.7404_{-0.0028}^{+0.0034}$ & {$[0.7376,0.7438]$} \\
\hline $\operatorname{Ar}(\mathbf{n}, *)$ & $0.523_{-0.041}^{+0.063}$ & {$[0.48,0.59]$} \\
\hline $\mathrm{Fe}(\mathrm{n}, *)$ & $0.5472_{-0.0059}^{+0.0054}$ & {$[0.541,0.553]$} \\
\hline $\operatorname{Cr}(\mathrm{n}, *)$ & $0.3989_{-0.0081}^{+0.0069}$ & {$[0.391,0.406]$} \\
\hline $\mathrm{Ni}(\mathrm{n}, *)$ & $0.5629_{-0.0095}^{+0.0086}$ & {$[0.553,0.572]$} \\
\hline $\operatorname{Mn}(\mathbf{n}, *)$ & $3.61_{-0.19}^{+0.17}$ & {$[3.42,3.79]$} \\
\hline $\mathrm{Cl}(\mathrm{n}, *)$ & $0.755_{-0.024}^{+0.025}$ & {$[0.731,0.780]$} \\
\hline $\mathrm{O}(\mathbf{n}, *)$ & $0.000_{-0.000}^{+0.044}$ & {$[0.000,0.044]$} \\
\hline $\mathbf{I}(\mathrm{n}, *)$ & 1 & (fixed) \\
\hline $\mathrm{Cu}(\mathrm{n}, *)$ & $0.452_{-0.022}^{+0.025}$ & {$[0.430,0.476]$} \\
\hline $\mathrm{C}(\mathbf{n}, \gamma)$ & $0.556_{-0.092}^{+0.089}$ & {$[0.46,0.65]$} \\
\hline Other (fixed) & 1 & (fixed) \\
\hline Other $\left(\mathbf{n}, \mathbf{n}^{\prime} \gamma\right)$ & 1 & (fixed) \\
\hline $\mathbf{C}(\mathbf{n}, \mathbf{n} ’ \gamma)$ & $7.03_{-0.57}^{+0.46}$ & {$[6.5,7.5]$} \\
\hline Multiple interactions & 1 & (fixed) \\
\hline 4.7 $\mathrm{MeV}{ }^{40} \mathrm{Ar} \gamma$ & $1.35_{-0.23}^{+0.19}$ & {$[1.12,1.54]$} \\
\hline $7.6 \mathrm{MeV}{ }^{56} \mathrm{Fe} \gamma$ & $0.964_{-0.052}^{+0.067}$ & {$[0.91,1.03]$} \\
\hline
\end{tabular}

Table 7.5: Complete AmBe calibration fit results. The second and third columns contain the same information. 

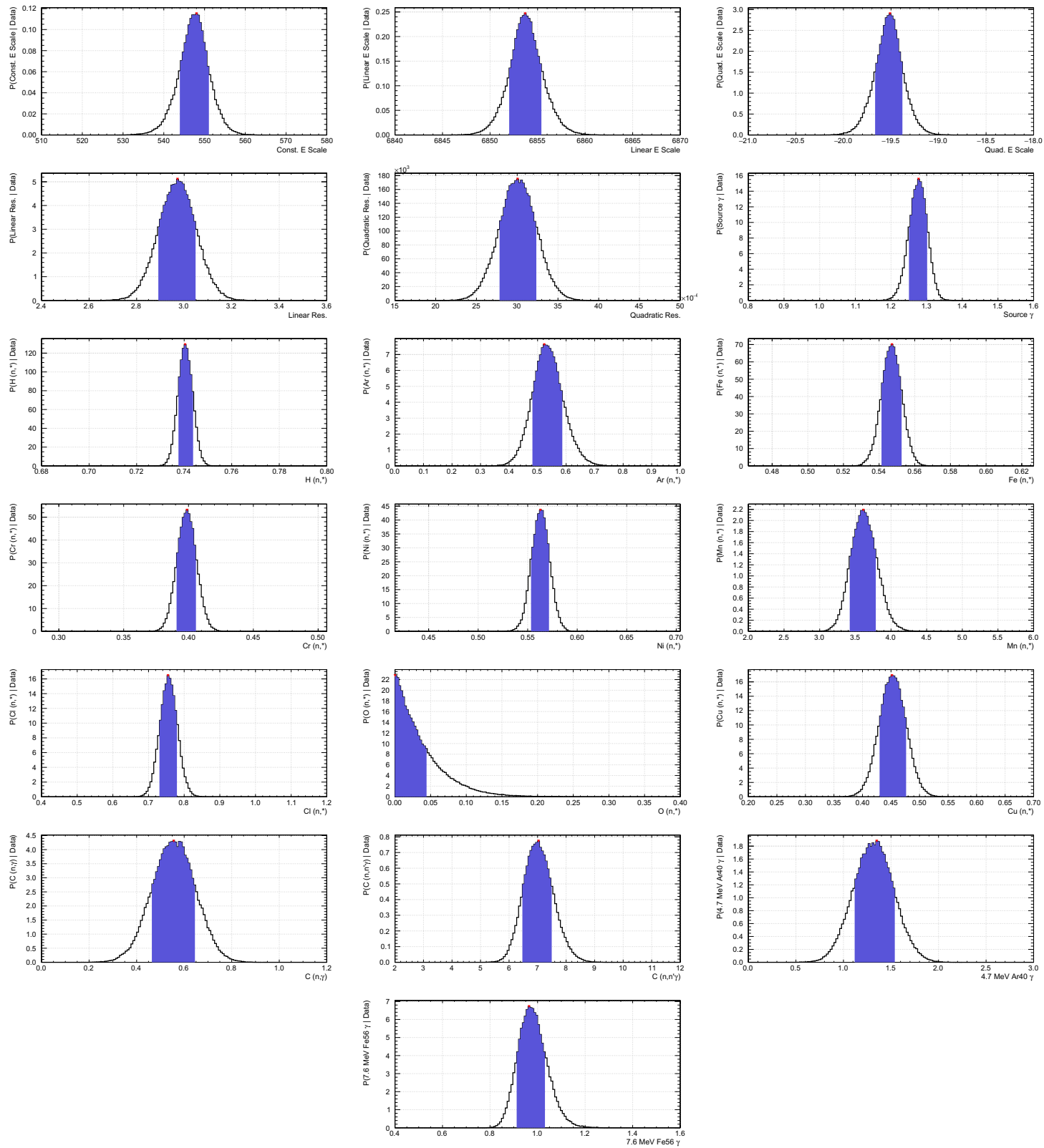

Figure 7.36: Fully marginalized posteriors from the AmBe calibration fit. 


\begin{tabular}{|c|c|}
\hline Components & Correlation \\
\hline Const. E Scale and Quad. E Scale & 0.909926 \\
\hline Const. E Scale and Linear E Scale & -0.947699 \\
\hline Linear E Scale and Quad. E Scale & -0.992485 \\
\hline Linear Res. and Quadratic Res. & -0.872532 \\
\hline Source $\gamma$ and $7.6 \mathrm{MeV}{ }^{56} \mathbf{F e} \gamma$ & 0.395623 \\
\hline Source $\gamma$ and $\mathbf{C}(\mathbf{n}, \mathbf{n} ' \gamma)$ & -0.951319 \\
\hline Source $\gamma$ and $\mathbf{C l}(\mathbf{n}, *)$ & -0.416607 \\
\hline Source $\gamma$ and $\mathbf{F e}(\mathbf{n}, *)$ & -0.306847 \\
\hline $\operatorname{Ar}\left(\mathbf{n},{ }^{*}\right)$ and $7.6 \mathrm{MeV}{ }^{56} \mathbf{F e} \gamma$ & 0.392365 \\
\hline $\operatorname{Ar}\left(\mathbf{n},{ }^{*}\right)$ and $4.7 \mathrm{MeV}{ }^{40} \mathrm{Ar} \gamma$ & -0.623537 \\
\hline $\operatorname{Ar}(\mathbf{n}, *)$ and $\mathbf{O}(\mathbf{n}, *)$ & -0.408656 \\
\hline $\operatorname{Ar}(\mathbf{n}, *)$ and $\operatorname{Fe}(\mathbf{n}, *)$ & -0.309925 \\
\hline Fe $\left(\mathbf{n},{ }^{*}\right)$ and $7.6 \mathrm{MeV}{ }^{56} \mathbf{F e} \gamma$ & -0.798819 \\
\hline $\mathbf{F e}\left(\mathbf{n},{ }^{*}\right)$ and $\mathbf{M n}\left(\mathbf{n},{ }^{*}\right)$ & -0.371181 \\
\hline $\mathrm{Cr}(\mathbf{n}, *)$ and $\mathbf{C u}(\mathbf{n}, *)$ & -0.35668 \\
\hline $\operatorname{Cr}(\mathbf{n}, *)$ and $\mathrm{Ni}(\mathbf{n}, *)$ & -0.4624 \\
\hline $\operatorname{Mn}\left(\mathbf{n},{ }^{*}\right)$ and $7.6 \mathrm{MeV}^{56} \mathbf{F e} \gamma$ & 0.486544 \\
\hline $\mathbf{M n}\left(\mathbf{n},{ }^{*}\right)$ and $\mathbf{C}(\mathbf{n}, \gamma)$ & -0.334238 \\
\hline $\operatorname{Mn}(\mathbf{n}, *)$ and $\mathrm{Cl}(\mathbf{n}, *)$ & -0.499712 \\
\hline $\mathbf{C l}(\mathbf{n}, *)$ and $\mathbf{C}\left(\mathbf{n}, \mathbf{n}^{\prime} \gamma\right)$ & 0.382068 \\
\hline $\mathbf{C}\left(\mathbf{n}, \mathbf{n}^{\prime} \gamma\right)$ and $7.6 \mathrm{MeV}^{56} \mathbf{F e} \gamma$ & -0.318792 \\
\hline
\end{tabular}

Table 7.6: Table of all correlations in the full AmBe calibration fit with a magnitude greater than 0.3. For a table of all correlations, see Figure C.1. 

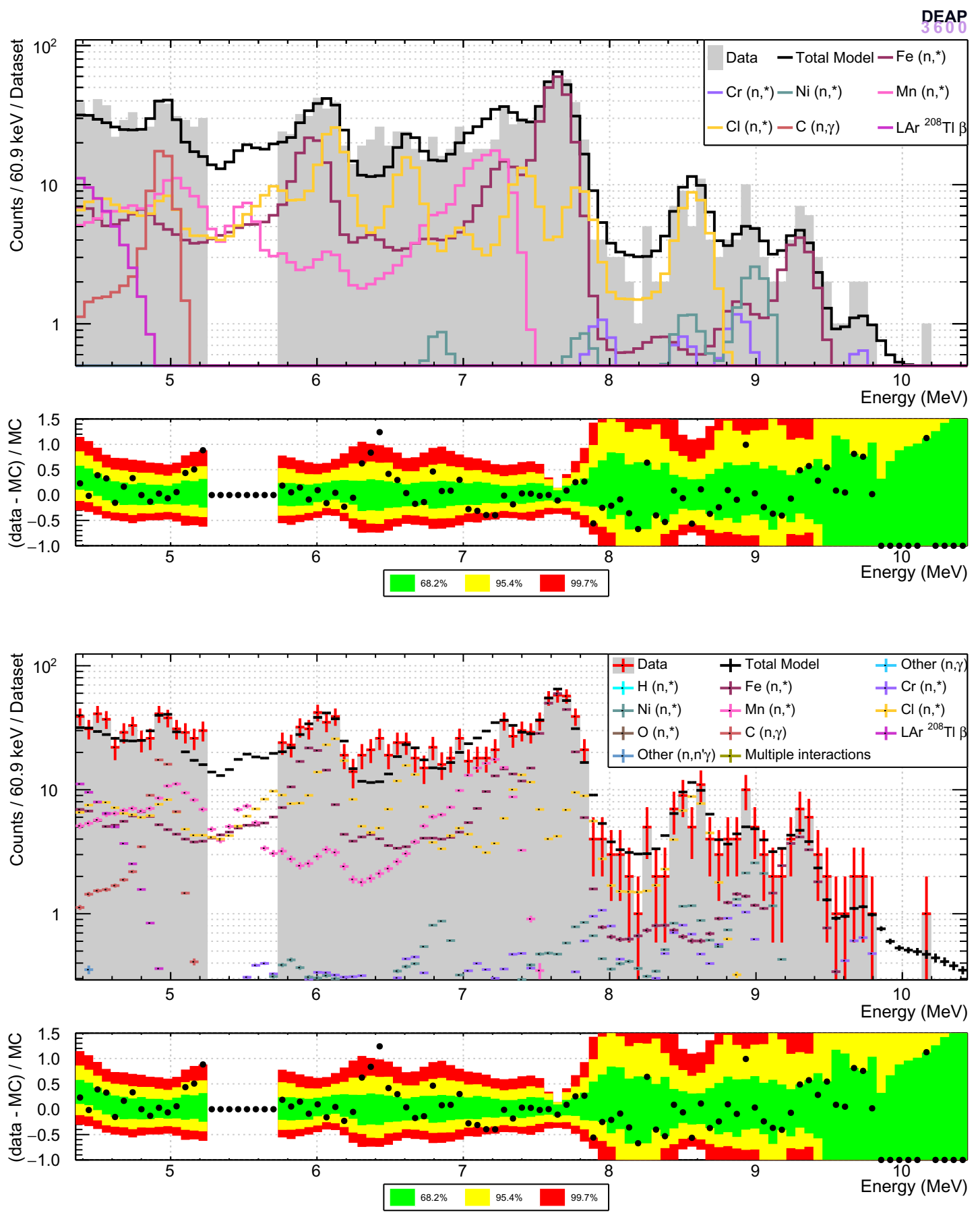

Figure 7.37: Final fit of the physics data prior to unblinding. P-value $=0.016$.

Note that the two plots are the same, but with different plotting style. 


\begin{tabular}{|c|c|c|}
\hline Parameter & Best fit value & $68 \%$ credibility interval \\
\hline Const. E Scale & $548.7_{-5.0}^{+2.1}$ & {$[543.7,550.8]$} \\
\hline Linear E Scale & $6853.7_{-1.8}^{+1.7}$ & {$[6851.9,6855.4]$} \\
\hline Quad. E Scale & $-19.51_{-0.16}^{+0.13}$ & {$[-19.66,-19.38]$} \\
\hline Linear Res. & $2.983_{-0.079}^{+0.078}$ & {$[2.90,3.06]$} \\
\hline Quadratic Res. & $0.0000305_{-0.0000021}^{+0.0000024}$ & {$[0.0000284,0.0000329]$} \\
\hline Other $(\mathbf{n}, \gamma)$ & 1 & (fixed) \\
\hline $\mathbf{H}(\mathbf{n}, *)$ & 1 & (fixed) \\
\hline $\operatorname{Ar}(\mathbf{n}, *)$ & $0.00_{-0.00}^{+0.19}$ & {$[0.00,0.19]$} \\
\hline $\mathrm{Fe}(\mathrm{n}, *)$ & $0.795_{-0.059}^{+0.068}$ & {$[0.74,0.86]$} \\
\hline $\operatorname{Cr}(n, *)$ & $0.108_{-0.066}^{+0.071}$ & {$[0.04,0.18]$} \\
\hline $\mathrm{Ni}(\mathrm{n}, *)$ & $0.20_{-0.10}^{+0.11}$ & {$[0.10,0.31]$} \\
\hline $\operatorname{Mn}(\mathbf{n}, *)$ & $7.33_{-0.89}^{+1.22}$ & {$[6.4,8.5]$} \\
\hline $\mathrm{Cl}(\mathrm{n}, *)$ & $0.97_{-0.13}^{+0.11}$ & {$[0.84,1.08]$} \\
\hline $\mathrm{O}(\mathbf{n}, *)$ & 1 & (fixed) \\
\hline $\mathrm{Cu}(\mathrm{n}, *)$ & $0.00_{-0.00}^{+0.10}$ & {$[0.00,0.10]$} \\
\hline $\mathbf{C}(\mathbf{n}, \gamma)$ & $1.65_{-0.45}^{+0.38}$ & {$[1.20,2.03]$} \\
\hline $\mathrm{B}(\mathrm{n}, *)$ & 1 & (fixed) \\
\hline Other (fixed) & 1 & (fixed) \\
\hline $\mathbf{A V}{ }^{208} \mathbf{T l} \beta$ & $0_{-0}^{+50}$ & {$[0,50]$} \\
\hline $\mathbf{L A r}{ }^{208} \mathbf{T l} \beta$ & $0.0203_{-0.0110}^{+0.0092}$ & {$[0.009,0.030]$} \\
\hline Other $\left(\mathbf{n}, \mathbf{n}^{\prime} \gamma\right)$ & 1 & (fixed) \\
\hline $\mathbf{C}\left(\mathbf{n}, \mathbf{n}^{\prime} \gamma\right)$ & $0.0_{-0.0}^{+2.7}$ & {$[0.0,2.7]$} \\
\hline Multiple interactions & 1 & (fixed) \\
\hline 4.7 $\mathrm{MeV}{ }^{40} \mathrm{Ar} \gamma$ & $1.00_{-0.20}^{+0.21}$ & {$[0.80,1.21]$} \\
\hline $7.6 \mathrm{MeV}{ }^{56} \mathrm{Fe} \gamma$ & $0.72_{-0.16}^{+0.30}$ & {$[0.56,1.02]$} \\
\hline
\end{tabular}

Table 7.7: Best fit parameters for the final blinded physics fit (Figure 7.37). The second and third columns contain the same information. 

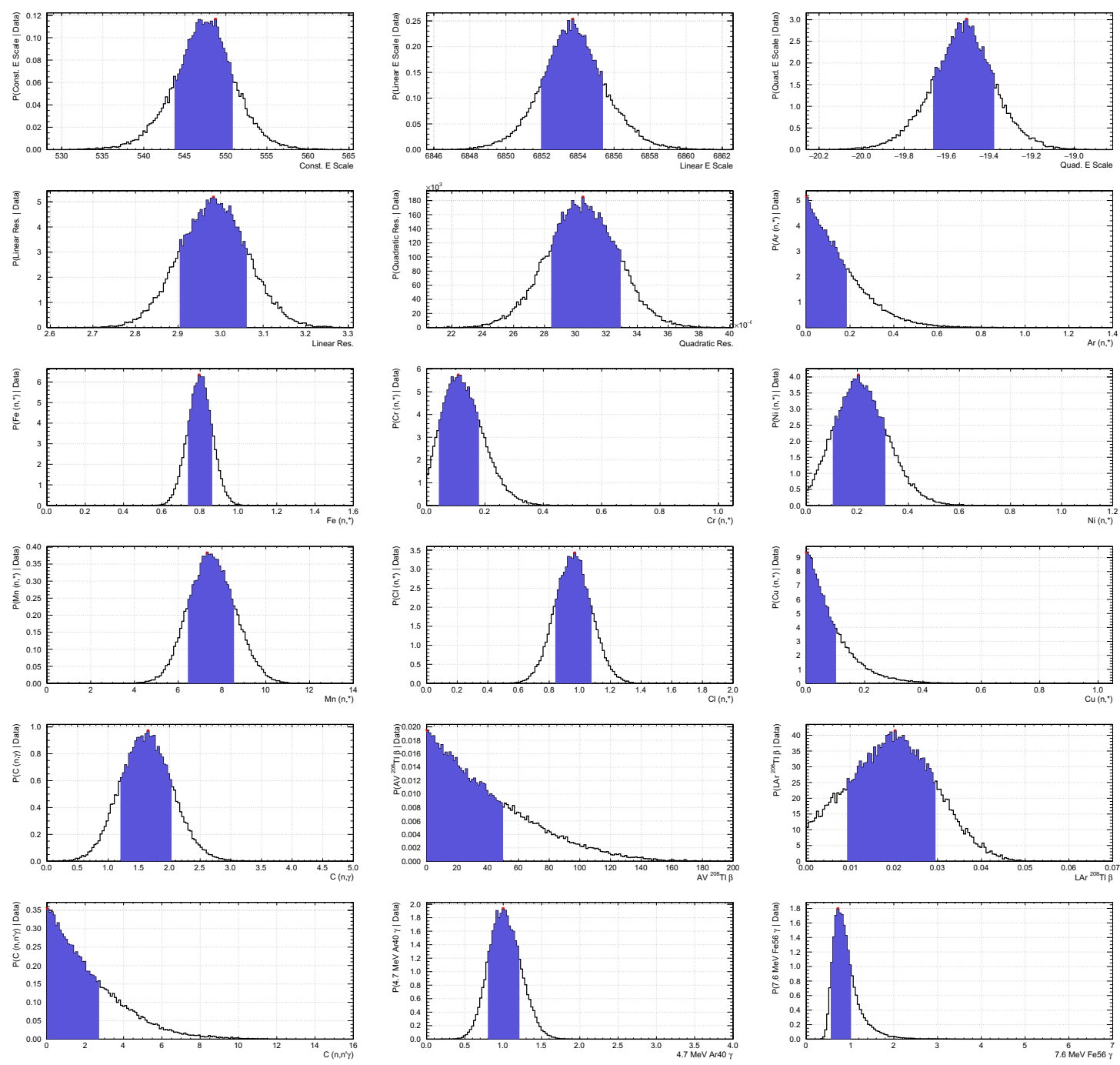

Figure 7.38: Fully marginalized posteriors from the blinded physics fit.

\begin{tabular}{|c|c|}
\hline Components & Correlation \\
\hline $\mathbf{A r}\left(\mathbf{n},{ }^{*}\right)$ and $\mathbf{7 . 6} \mathbf{~ M e V}{ }^{56} \mathbf{F e} \gamma$ & 0.322464 \\
$\mathbf{F e}\left(\mathbf{n},{ }^{*}\right)$ and $\mathbf{7 . 6} \mathbf{~ M e V}{ }^{56} \mathbf{F e} \gamma$ & -0.396486 \\
$\mathbf{F e}\left(\mathbf{n},{ }^{*}\right)$ and $\mathbf{C l}\left(\mathbf{n},{ }^{*}\right)$ & -0.442914 \\
$\mathbf{C r}\left(\mathbf{n},{ }^{*}\right)$ and $\mathbf{N i}\left(\mathbf{n},{ }^{*}\right)$ & -0.461036 \\
$\mathbf{M n}\left(\mathbf{n},{ }^{*}\right)$ and $\mathbf{C ~}(\mathbf{n}, \gamma)$ & -0.310544 \\
$\mathbf{A V}{ }^{208} \mathbf{T l} \beta$ and $\mathbf{L A r}{ }^{208} \mathbf{T l} \beta$ & -0.591211 \\
\hline
\end{tabular}

Table 7.8: Table of all correlations in the final blinded physics fit with a magnitude greater than 0.3. For a table of all correlations, see Figure C.2. 


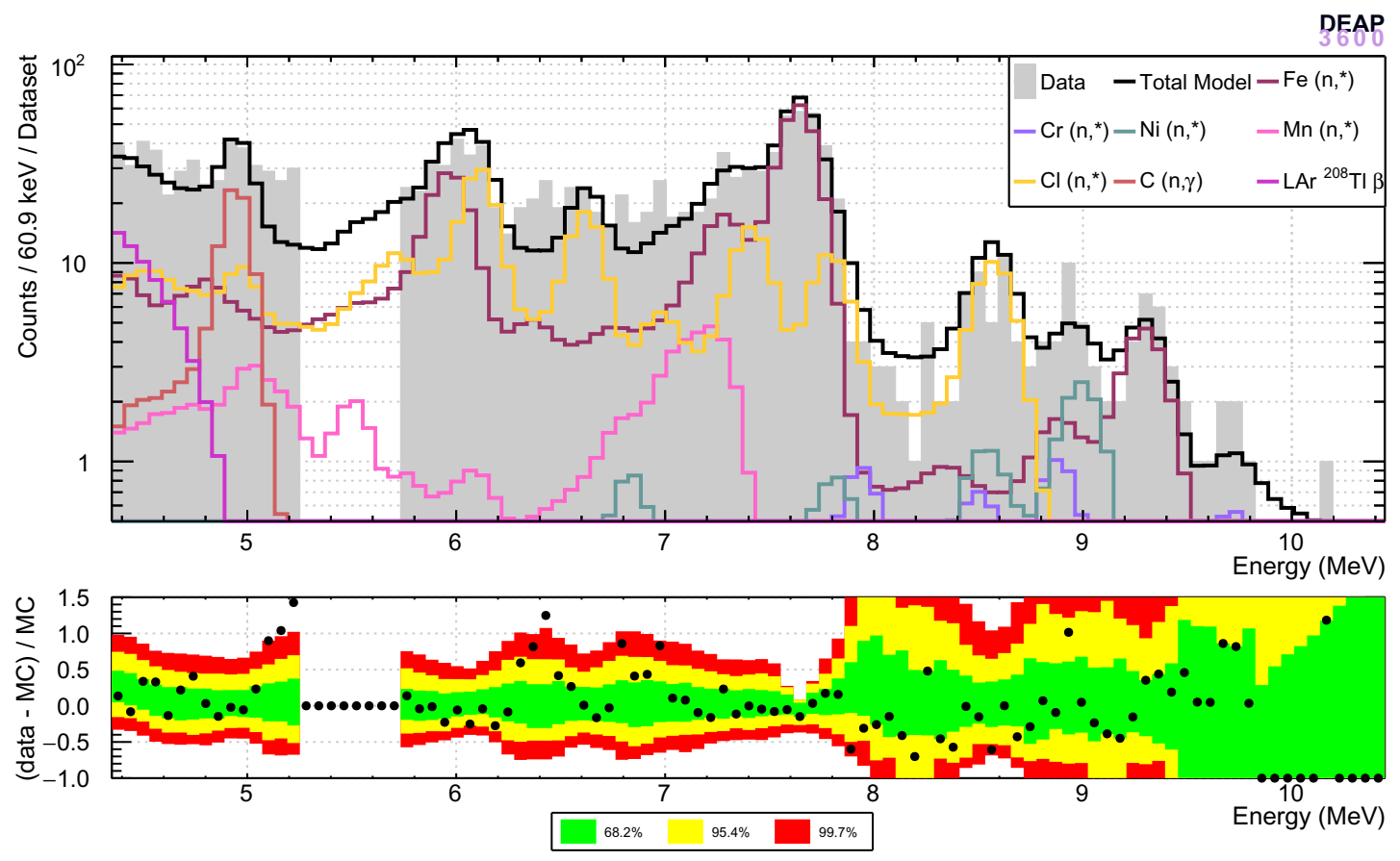

Figure 7.39: Blinded physics fit with the Mn normalization fixed to 2 times the GEANT4 prediction. P-value: 0.00008 . 


\begin{tabular}{|c|c|c|}
\hline Parameter & Best fit value & $68 \%$ credibility interval \\
\hline Const. E Scale & $547.5_{-3.6}^{+3.6}$ & {$[543.8,551.1]$} \\
\hline Linear E Scale & $6853.1_{-1.2}^{+2.5}$ & {$[6851.8,6855.5]$} \\
\hline Quad. E Scale & $-19.48_{-0.19}^{+0.10}$ & {$[-19.67,-19.38]$} \\
\hline Linear Res. & $2.982_{-0.081}^{+0.076}$ & {$[2.90,3.06]$} \\
\hline Quadratic Res. & $0.0000310_{-0.0000027}^{+0.0000016}$ & {$[0.0000283,0.0000326]$} \\
\hline Other $(\mathbf{n}, \gamma)$ & 1 & (fixed) \\
\hline $\mathbf{H}(\mathbf{n}, *)$ & 1 & (fixed) \\
\hline $\operatorname{Ar}(\mathbf{n}, *)$ & $0.000_{-0.000}^{+0.090}$ & {$[0.000,0.090]$} \\
\hline $\mathrm{Fe}(\mathrm{n}, *)$ & $0.892_{-0.068}^{+0.068}$ & {$[0.82,0.96]$} \\
\hline $\operatorname{Cr}(n, *)$ & $0.094_{-0.059}^{+0.079}$ & {$[0.04,0.17]$} \\
\hline $\mathrm{Ni}(\mathrm{n}, *)$ & $0.20_{-0.10}^{+0.11}$ & {$[0.10,0.31]$} \\
\hline $\operatorname{Mn}(\mathbf{n}, *)$ & 2 & (fixed) \\
\hline $\mathrm{Cl}(\mathrm{n}, *)$ & $1.11_{-0.11}^{+0.12}$ & {$[1.00,1.23]$} \\
\hline $\mathrm{O}(\mathrm{n}, *)$ & 1 & (fixed) \\
\hline $\mathrm{Cu}(\mathrm{n}, *)$ & $0.018_{-0.018}^{+0.185}$ & {$[0.00,0.20]$} \\
\hline $\mathbf{C}(\mathbf{n}, \gamma)$ & $2.20_{-0.31}^{+0.52}$ & {$[1.89,2.72]$} \\
\hline $\mathrm{B}(\mathrm{n}, *)$ & 1 & (fixed) \\
\hline Other (fixed) & 1 & (fixed) \\
\hline $\mathbf{A V}{ }^{208} \mathbf{T l} \beta$ & $0_{-0}^{+43}$ & {$[0,43]$} \\
\hline LAr ${ }^{208} \mathbf{T l} \beta$ & $0.0259_{-0.0109}^{+0.0086}$ & {$[0.015,0.035]$} \\
\hline Other $\left(\mathbf{n}, \mathbf{n}^{\prime} \gamma\right)$ & 1 & (fixed) \\
\hline $\mathbf{C}\left(\mathbf{n}, \mathbf{n}^{\prime} \gamma\right)$ & $0.16_{-0.16}^{+2.46}$ & {$[0.0,2.6]$} \\
\hline Multiple interactions & 1 & (fixed) \\
\hline 4.7 $\mathrm{MeV}{ }^{40} \mathrm{Ar} \gamma$ & $0.96_{-0.14}^{+0.24}$ & {$[0.81,1.20]$} \\
\hline $7.6 \mathrm{MeV}{ }^{56} \mathrm{Fe} \gamma$ & $0.54_{-0.10}^{+0.15}$ & {$[0.44,0.69]$} \\
\hline
\end{tabular}

Table 7.9: Best fit parameters for the blinded physics fit with the Mn normalization fixed to 2, as plotted in Figure 7.39 . 


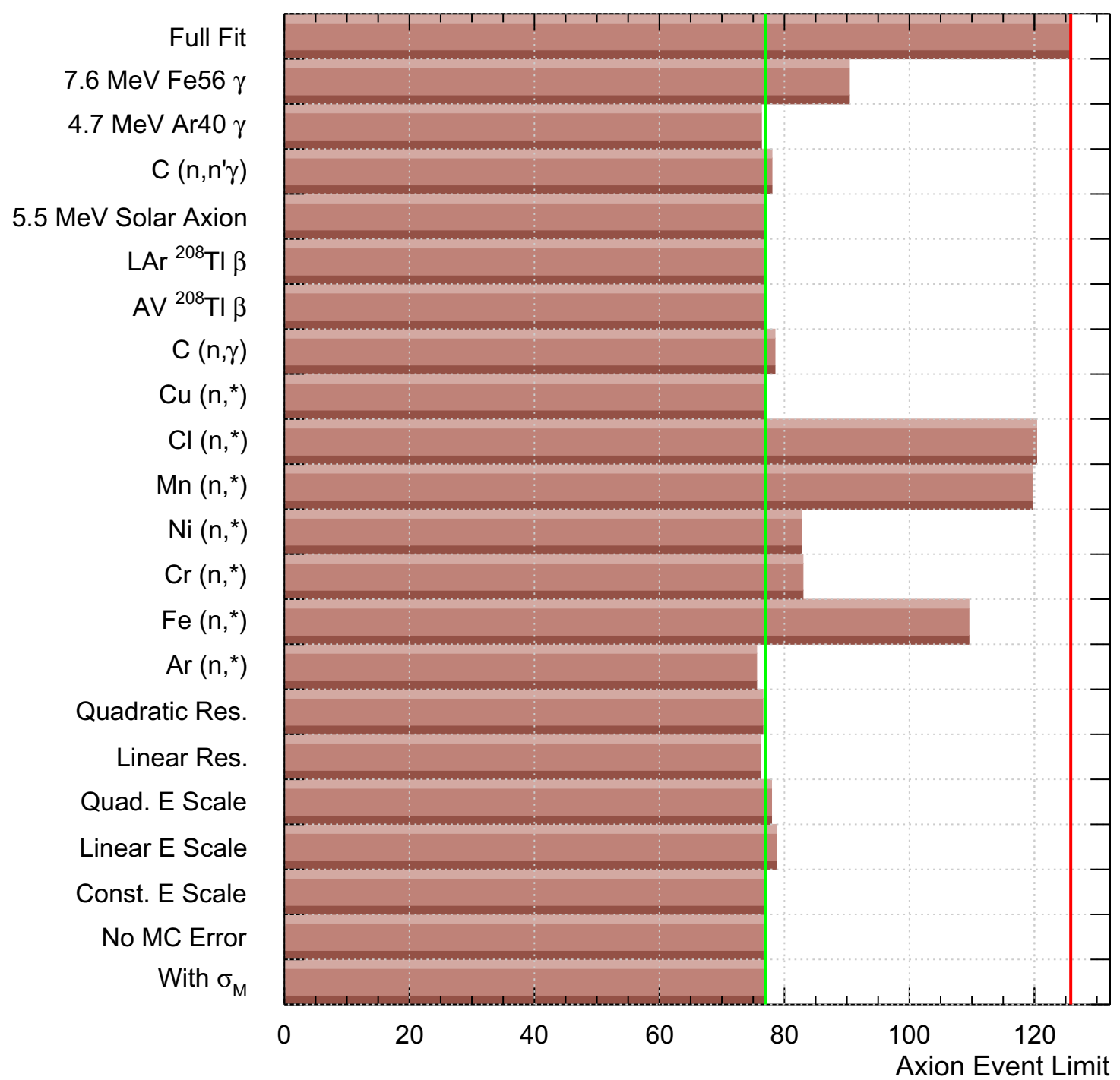

Figure 7.40: Limits on the number of axion interactions obtained through fits performed on a single toy dataset derived from the best physics fit result, with a factor of $\mathbf{1 0}$ more statistics than in the real data. All the parameters were fixed except the $5.5 \mathrm{MeV}$ Solar Axion normalization and the parameter listed on the Y-axis. In the "No MC Error" and "With $\sigma_{M}$ " cases, only the axion normalization was free to float. For the former case, the MC bin error was ignored by the fitter. In the latter case, the uncertainty on the detector mass was included. In the "Full Fit" case, all parameters were free to float. The green line labels the limit obtained with only the axion normalization free to float, and the red line labels the limit obtained when all parameters are free. 

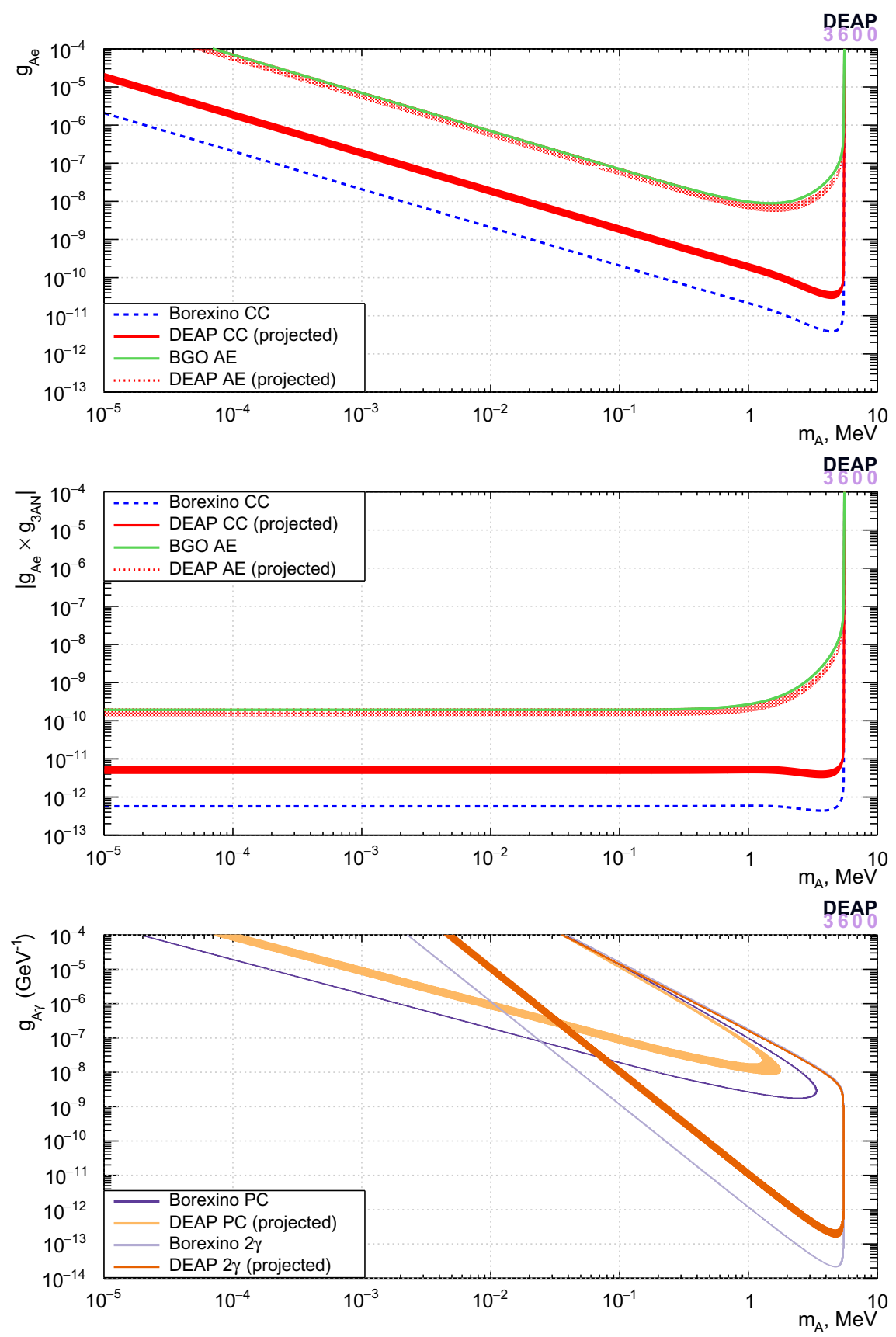

Figure 7.41: Projected DEAP exclusions (90\% CL) overlaid with exclusion curves from the two leading experimental searches for $5.5 \mathrm{MeV}$ solar axions [49], [48]. The thickness of the DEAP curve gives the range of possible limits based on the standard deviation of $90 \%$ confidence limits obtained when fitting to toy datasets (see table 7.4). Top: The limit on the axion-electron coupling in the hadronic axion model. Middle: The limit on the product of the axion-electron and the axion-neutron couplings in the more general GUT model. Bottom: The limit on the axion-photon coupling constant in the hadronic axion model. 


\section{Chapter 8}

\section{Conclusion}

Three separate analyses were discussed in this thesis - a study of $\alpha$ decay backgrounds, a study of radiogenic neutron backgrounds, and a search for $5.5 \mathrm{MeV}$ solar axions.

Cuts were developed to select populations of $\alpha$ decays in the data. Since $\alpha$ decays outside the main LAr volume will yield a lower energy signature than decays in the main LAr volume, a time coincidence tagging method was employed to study the rate and locations of events that produce less PE in the PMTs. This method was used in particular in studying the rate of ${ }^{214}$ Po decays on the inner detector surface, with the numbers of suspected ${ }^{214}$ Po decays in the 231 day dataset given in table 5.16 . The locations of these events were plotted in Figure 5.28, showing that these events seem to be inhomogeneously distributed on the detector surface. The time-coincidence

tagging method was also used to check for evidence of ${ }^{214}$ Po decays on the surface of the neck of the detector. ${ }^{220} \mathrm{Rn}$ and ${ }^{216} \mathrm{Po}$ were also studied, and it was shown that these too contain degraded energy events.

Since this method makes it possible to verify whether a population of events contains ${ }^{214}$ Po decays, it was also used to understand the nature of what may be $\alpha$ decays on dust particles within the main LAr volume. Evidence was presented that seems to favour the dust interpretation of these anomalous events. Work is underway within the collaboration to remove any dust from within the detector. 
A multi-component fit was performed on most of the $\alpha$ decay energy spectrum. This fit required the development of a correction to the event energy estimator due to non-linear PMT and digitizer effects. The final fit results are in table 5.4 and Figure 5.7. ${ }^{212}$ Po was not directly included in the fit, but was studied with a separate side-band analysis. Since this work was performed, a more sophisticated saturation correction was developed at the waveform level, which is expected to simplify future measurements of the $\alpha$ background rates. The results of this fit were included in the latest DEAP-3600 WIMP search analysis [1].

The radiogenic neutron background was estimated for different cut flows as part of the unblinding process for the full second fill dataset. The results are in table 6.3 . and will be included in the upcoming DEAP-3600 WIMP search result on the full DEAP-3600 dataset.

A search for $5.5 \mathrm{MeV}$ solar axions is currently in progress, and very close to completion. This is a blinded study, with unblinding pending a proper understanding of an excess in the background model in the side-band at $6.4 \mathrm{MeV}$. Fits have been performed on the AmBe calibration data to constrain the energy response parameters for use in the fit to the full physics dataset. The physics dataset has been fit, excluding the axion ROI.

DEAP-3600 is currently undergoing an upgrade to reduce the amount of dust within the active detector volume, coat the neck with a different wavelength shifter to provide discrimination power for neck events and remove the liquid argon layer from the neck. This is expected to remove all the major backgrounds to the WIMP search, allowing for much greater sensitivity to WIMP interactions.

Research has already begun towards the design of ARGO, a multi-hundred tonne successor to DEAP-3600. This experiment will draw on the knowledge gained by the DEAP and DarkSide collaborations. In addition to the WIMP search, there are plans to repeat the axion search analysis detailed in this report and to obtain a significantly 
more sensitive $5.5 \mathrm{MeV}$ axion search result. 


\section{References}

[1] R. Ajaj et al., Phys. Rev. D 100, 022004 (2019).

[2] P.-A. Amaudruz et al., Astroparticle Physics 108, 1 (2019).

[3] D. J. Griffiths, Introduction to elementary particles; 2nd rev. version, Physics textbook (Wiley, New York, NY, 2008).

[4] B. Paczynski, The Astrophysical Journal 304, 1 (1986).

[5] F. Zwicky, Astrophysical Journal 86, 217 (1937).

[6] F. Zwicky, Phys. Rev. 61, 489 (1942).

[7] V. C. Rubin, W. K. Ford Jr, and N. Thonnard, The Astrophysical Journal 225, L107 (1978).

[8] V. C. Rubin, J. Ford, W. K., and N. Thonnard, Astrophysical Journal 238, 471 (1980).

[9] V. C. Rubin, J. Ford, W. K., N. Thonnard, and D. Burstein, Astrophysical Journal 261, 439 (1982).

[10] V. C. Rubin, D. Burstein, J. Ford, W. K., and N. Thonnard, Astrophysical Journal 289, 81 (1985).

[11] M. Markevitch et al., The Astrophysical Journal 606, 819 (2004).

[12] Planck Collaboration, A\&A 641, A6 (2020).

[13] K. Jedamzik and M. Pospelov, New Journal of Physics 11, 105028 (2009).

[14] G. R. Blumenthal, S. M. Faber, J. R. Primack, and M. J. Rees, Nature 311, 517 (1984). 
[15] S. P. Martin, in Perspectives on Supersymmetry (World Scientific, Singapore, 1998), pp. 1-98.

[16] R. Peccei and H. R. Quinn, Phys. Rev. Lett. 38, 1440 (1977).

[17] L. D. Duffy and K. van Bibber, New Journal of Physics 11, 105008 (2009).

[18] C. Alcock et al., The Astrophysical Journal 542, 281 (2000).

[19] Tisserand, P. et al., A\&A 469, 387 (2007).

[20] D. S. Graff and K. Freese, The Astrophysical Journal Letters 456, L49 (1996).

[21] J. Lesgourgues and S. Pastor, Advances in High Energy Physics 2012, 608515 (2012).

[22] M. Kamionkowski, in High Energy Physics and Cosmology, 1997 Summer School, edited by E. Gava et al. (World Scientific, Singapore, 1998), Vol. 14, p. 394.

[23] L. Roszkowski, E. M. Sessolo, and S. Trojanowski, Reports on Progress in Physics 81, 066201 (2018).

[24] C. S. Wu et al., Phys. Rev. 105, 1413 (1957).

[25] C.-T. Chan, arXiv:hep-ph/9704427 .

[26] K. Abe et al., Nature 580, 339 (2020).

[27] C. Abel et al., Phys. Rev. Lett. 124, 081803 (2020).

[28] S. Dar, arXiv:hep-ph/0008248 .

[29] A. S. Zhevlakov, T. Gutsche, and V. E. Lyubovitskij, Phys. Rev. D 99, 115004 (2019).

[30] Zhevlakov, Alexey S., Gutsche, Thomas, and Lyubovitskij, Valery E., EPJ Web Conf. 212, 07005 (2019).

[31] M. Dine, L. S. Haskins, L. Ubaldi, and D. Xu, Journal of High Energy Physics 2018, 1 (2018).

[32] S. Weinberg, Phys. Rev. Lett. 40, 223 (1978).

[33] F. Wilczek, Phys. Rev. Lett. 40, 279 (1978).

[34] T. Donnelly et al., Physical Review D 18, 1607 (1978). 
[35] J. E. Kim, Phys. Rev. Lett. 43, 103 (1979).

[36] M. Shifman, A. Vainshtein, and V. Zakharov, Nuclear Physics B 166, 493 (1980).

[37] M. Dine, W. Fischler, and M. Srednicki, Physics Letters B 104, 199 (1981).

[38] A. R. Zhitnitskij, Yadernaya Fizika 31, 497 (1980).

[39] A. Bischoff-Kim, M. H. Montgomery, and D. E. Winget, The Astrophysical Journal 675, 1512 (2008).

[40] Z. Berezhiani, L. Gianfagna, and M. Giannotti, Physics Letters B 500, 286 (2001).

[41] L. J. Hall and T. Watari, Phys. Rev. D 70, 115001 (2004).

[42] S. J. Asztalos et al., Phys. Rev. Lett. 104, 041301 (2010).

[43] C. Abel et al., Phys. Rev. X 7, 041034 (2017).

[44] J. N. Bahcall, M. C. Gonzalez-Garcia, and C. Peña Garay, Phys. Rev. Lett. 90, 131301 (2003).

[45] K. R. Lang, Essential astrophysics (Springer, Berlin, 2013).

[46] A. M. Serenelli, W. C. Haxton, and C. Peña-Garay, The Astrophysical Journal 743, 24 (2011).

[47] G. Schmid et al., Physical Review C 56, 2565 (1997).

[48] A. V. Derbin et al., The European Physical Journal C 74, 3035 (2014).

[49] G. Bellini et al., Phys. Rev. D 85, 092003 (2012).

[50] A. V. Derbin, A. S. Kayunov, and V. N. Muratova, Bulletin of the Russian Academy of Sciences: Physics 74, 805 (2010).

[51] F. T. Avignone III et al., Phys. Rev. D 37, 618 (1988).

[52] S. S. Westerdale, Ph.D. thesis, Princeton University, 2016.

[53] N. D. C. Network, EXFOR Systems Manual: Nuclear Reaction Data Exchange Format, Brookhaven National Laboratory, U.S.A., 1996, compiled and edited by V. McLane. 
[54] R. Brun and F. Rademakers, Nucl. Instrum. Methods Phys. Res. A 389, 81 (1997), new Computing Techniques in Physics Research V.

[55] T. Bolton et al., RAT (is an Analysis Tool) User's Guide, 2018.

[56] S. Agostinelli et al., Nucl. Instrum. Methods Phys. Res. A 506, 250 (2003).

[57] G. Audi and A. Wapstra, Nuclear Physics A 595, 409 (1995).

[58] W. A. Rolke, A. M. Lopez, and J. Conrad, Nuclear Instruments and Methods in Physics Research Section A: Accelerators, Spectrometers, Detectors and Associated Equipment 551, 493 (2005).

[59] R. Ajaj et al., Physical Review D 100, 072009 (2019).

[60] A. Gelman et al., Statistical science 7, 457 (1992).

[61] D. A. Brown et al., Nuclear Data Sheets 148, 1 (2018).

[62] Database of Prompt Gamma Rays from Slow Neutron Capture for Elemental Analysis, Non-serial Publications (INTERNATIONAL ATOMIC ENERGY AGENCY, Vienna, 2007). 


\section{Appendix A}

\section{Cuts Used for $\alpha$ Studies}

The standard pileup cuts are:

- subevent $\mathrm{N}==1$

- $2350<$ trigTime $<2650$

- $100<$ timefitT0 $<5500$

The standard low level cuts are:

- $\mathrm{qPE}>0$

- (!(dtmTrigSrc \& 0x82))

- (!(calcut \& 0x31f8))

\section{LAr Fit Cuts}

Data selection cuts:

- overallDataCut $=\left(\right.$ Alphas_box || $\mathrm{Bi}^{214} \mathrm{Po} \_$ext $)+$pileup + lowlevel + fmaxpe

- pileup $=$ subevent $N==1 \& \&$ trigTime $>2350 \& \&$ trigTime $<2650 \& \&$ timefitT0 $>100 \& \&$ timefitT 05500 
- lowlevel $=(!($ dtmTrigSrc \& 0x82) $\& \&(!($ calcut \& 0x31f8) $)$

- fmaxpe $=$ fmaxpe $<0.75$

- Alphas_box = fprompt $<-1.9193 \mathrm{e}-6{ }^{*} \mathrm{qPE}+0.81199 \& \&$ fprompt $<9.4036 \mathrm{e}-6{ }^{*} \mathrm{qPE}+0.46123 \& \&$ fprompt $<2.8644 \mathrm{e}-4{ }^{*} \mathrm{qPE}+-6.6191 \& \&$ fprompt $>-2.6585 \mathrm{e}-6{ }^{*} \mathrm{qPE}+0.73984 \& \&$ fprompt $>7.4128 \mathrm{e}-6^{*} \mathrm{qPE}+0.37565$

- $\mathrm{Bi}^{214} \mathrm{Po} \_$ext $=$ fprompt $>$ qPE*-1.2353e-05 $+1.0743 \& \&$ fprompt $<\mathrm{qPE}^{*}-1.2230 \mathrm{e}-05+1.2497 \& \&$ fprompt $>$ qPE* $1.382 \mathrm{e}-05+4.7527 \mathrm{e}-3 \& \&$ fprompt $<7.4128 \mathrm{e}-6{ }^{*} \mathrm{qPE}+0.3756 \& \&$ fprompt $>0.6 \& \&$ qPE $<50000$

Monte Carlo selection cuts:

- foverallMCCut $=$ fbounds + fpileup + fmaxpe

- bounds $=$ fprompt $<0.8 \& \&$ fprompt $>0.6 \& \&$ qPE $>10000 \& \&$ qPE $<50000$

- fpileup $=$ subevent $\mathrm{N}==1 \& \&$ trigTime $>2350 \& \&$ trigTime $<2650 \& \&$ timefitT0 $>100 \& \&$ timefitT $0<5500$

- fmaxpe $=$ fmaxpe $<0.75$

\section{Box Cuts}

"222 Rn":

"fprompt $<0.00028644 * \mathrm{qPE}+-6.6191 \& \&$ fprompt $<-0.0000019193 *$ qPE + $0.81199 \& \&$ fprompt $>-0.0000026585 * \mathrm{qPE}+0.73984 \& \&(\operatorname{sqrt}(\operatorname{mblikelihoodX} * * 2$ + mblikelihoodY**2 + mblikelihoodZ**2) / 851)**4.5 $<-0.00015433 *{ }^{*} \mathrm{PE}+$ $4.9400 \& \&\left(\operatorname{sqrt}\left(\operatorname{mblikelihoodX}{ }^{* *} 2+\operatorname{mblikelihoodY} \mathrm{Y}^{* *} 2+\operatorname{mblikelihoodZ}{ }^{* * 2}\right) /\right.$ $851)^{* *} 4.5>-0.00015851 * \mathrm{qPE}+4.5963 "$ 
"218Po":

$\left(\left({ }^{218} \mathrm{Po}_{-}{ }^{220} \mathrm{Rn} \_\right.\right.$box $\|{ }^{222} \mathrm{Rn} \_$box $) \& \&$ "(sqrt(mblikelihoodX ${ }^{* *} 2+\operatorname{mblikelihoodY} \mathrm{Y}^{* *} 2$ $+\operatorname{mblikelihoodZ**2)} / 851)^{* *} 4.5<-0.0001885089 * \mathrm{nSC}+5.6129618802 \& \&$ $\left(\operatorname{sqrt}\left(\operatorname{mblikelihoodX} X^{* *} 2+\operatorname{mblikelihood} Y^{* *} 2+\operatorname{mblikelihoodZ**2}\right) / 851\right)^{* *} 4.5>$ $-0.0001885089 * \mathrm{nSC}+5.2138867995 ")$

"214 Po_box"

fprompt $<0.0000074128 * \mathrm{qPE}+0.41857 \& \&$ fprompt $>0.0000074128 * \mathrm{qPE}+$ $0.37565 \& \&$ fprompt $<-0.0000019193 *$ qPE $+0.81199 \& \&$ fprompt $>-0.0000026585$ * $\mathrm{qPE}+0.73984$

Subcuts:

"222Rn_box":

"fprompt $>0.0000098130 *{ }^{\mathrm{qPE}}+0.41596 \& \&$ fprompt $<0.00028644 *{ }_{\mathrm{q}} \mathrm{PE}+$ $-6.6191 \& \&$ fprompt $<0.0000094036 *$ qPE $+0.46123 \& \&$ fprompt $<-0.0000019193$ * $\mathrm{qPE}+0.81199 \& \&$ fprompt $>-0.0000026585 * \mathrm{qPE}+0.73984 "$

"218Po_ ${ }^{220} \mathrm{Rn} \_$box"

"fprompt $>0.0000074128 *$ qPE $+0.45076 \& \&$ fprompt $<0.0000098130 *$ qPE + $0.41596 \& \&$ fprompt $<-0.0000019193 *$ qPE $+0.81199 \& \&$ fprompt $>-0.0000026585$ * qPE + 0.73984"

" ${ }^{220} \mathrm{Rn} \_$box":

"fprompt $<0.0000074128 *{ }_{\mathrm{qPE}}+0.45076 \& \&$ fprompt $>0.0000074128 *{ }_{\mathrm{qPE}}+$ $0.41857 \& \&$ fprompt $<-0.0000019193 *$ qPE $+0.81199 \& \&$ fprompt $>-0.0000026585$ * qPE + 0.73984" 


\section{Appendix B}

\section{Fits for $\alpha$ Systematics Study}
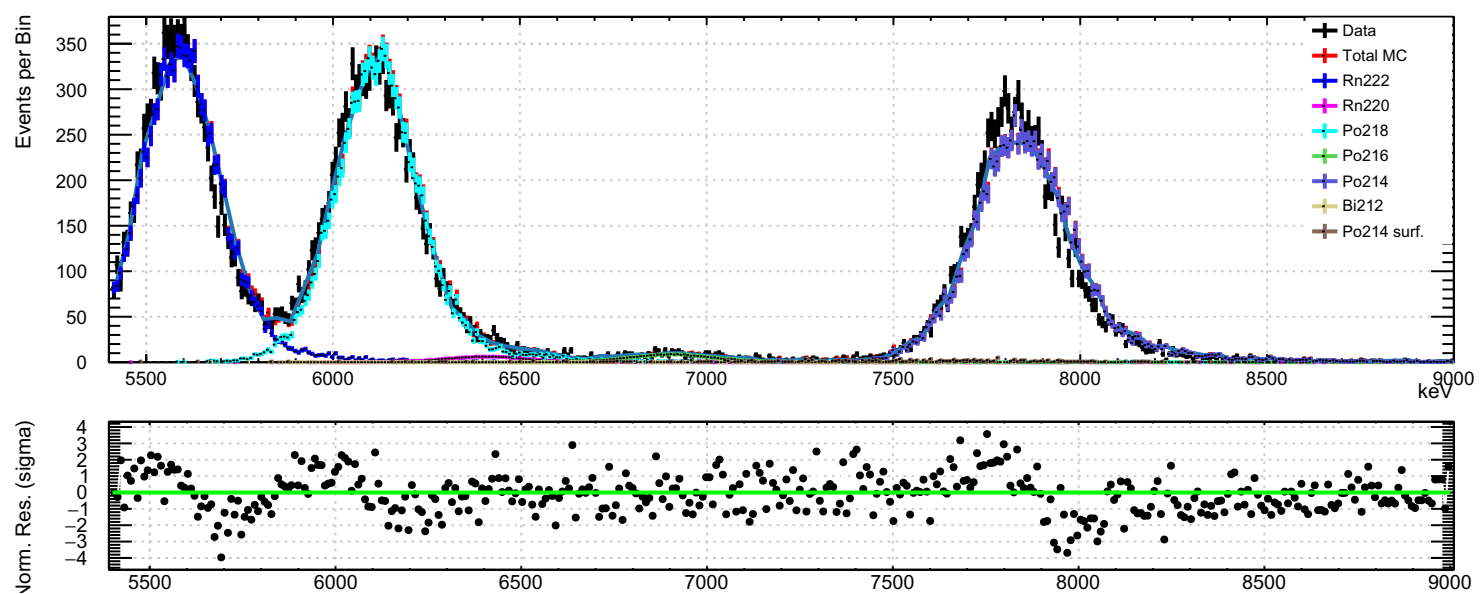

Figure B.1: Fit result when MC slopes in Figure 5.5 are shifted up by 1 sigma. See table B.1. $\chi^{2} / \mathrm{NDF}=1.62$ 


\begin{tabular}{|c|c|c|}
\hline Isotope & Activity (events $/ \mathbf{2 4 7 . 1 7}$ days) & Specific Activity $(\mu \mathrm{Bq} / \mathbf{k g})$ \\
\hline${ }^{222} \mathrm{Rn}$ & $10723.5_{-110.372}^{+110.871}$ & $0.153138_{-0.00475245}^{+0.00475482}$ \\
\hline${ }^{220} \mathrm{Rn}$ & $214.803_{-58.5828}^{+37.1822}$ & $0.00306753_{-0.000841409}^{+0.000538528}$ \\
\hline${ }^{218} \mathrm{Po}$ & $11170.7_{-147.838}^{+117.168}$ & $0.159526_{-0.00512549}^{+0.00496115}$ \\
\hline${ }^{216} \mathrm{Po}$ & $306.095_{-22.6149}^{+22.9738}$ & $0.00437125_{-0.00034739}^{+0.000352159}$ \\
\hline${ }^{214} \mathrm{Po}$ & $10677.6_{-114.001}^{+114.528}$ & $0.152484_{-0.00475188}^{+0.00475447}$ \\
\hline${ }^{212} \mathrm{Bi}$ & $1.53233 \mathrm{e}-07_{-0}^{+43.4351}$ & $2.18827 \mathrm{e}-12_{-6.40665 e-14}^{+0.000620283}$ \\
\hline${ }^{214} \mathrm{Po}$ surf. & $210.207_{-39.7719}^{+46.1331}$ events in fit range \\
\hline
\end{tabular}

Table B.1: Fit result when MC slopes in Figure 5.5 are shifted up by 1 sigma. See Figure B.1. $\chi^{2} / \mathrm{NDF}=1.62$

\begin{tabular}{|c|c|c|}
\hline Isotope & $\begin{array}{c}\text { Delta Activity } \\
\text { (events } / \mathbf{2 4 7 . 1 7} \text { days })\end{array}$ & $\begin{array}{c}\text { Delta Specific } \\
\text { Activity }(\mu \mathbf{B q} / \mathbf{k g})\end{array}$ \\
\hline${ }^{222} \mathrm{Rn}$ & -83.0926 & -0.00118662 \\
\hline${ }^{220} \mathrm{Rn}$ & -21.2899 & -0.000304035 \\
\hline${ }^{218} \mathrm{Po}$ & -68.2901 & -0.000975229 \\
\hline${ }^{216} \mathrm{Po}$ & -31.5032 & -0.000449888 \\
\hline${ }^{214} \mathrm{Po}$ & -152.865 & -0.00218302 \\
\hline${ }^{212} \mathrm{Bi}$ & $-5.08344 \mathrm{e}-05$ & $-7.2595 \mathrm{e}-10$ \\
\hline${ }^{214} \mathrm{Po}$ surf. & \multicolumn{2}{|c|}{198.683 events in fit range } \\
\hline
\end{tabular}

Table B.2: Fit result when MC slopes in Figure 5.5 are shifted up by 1 sigma. See Figure B.1. $\chi^{2} / \mathrm{NDF}=1.62$ 

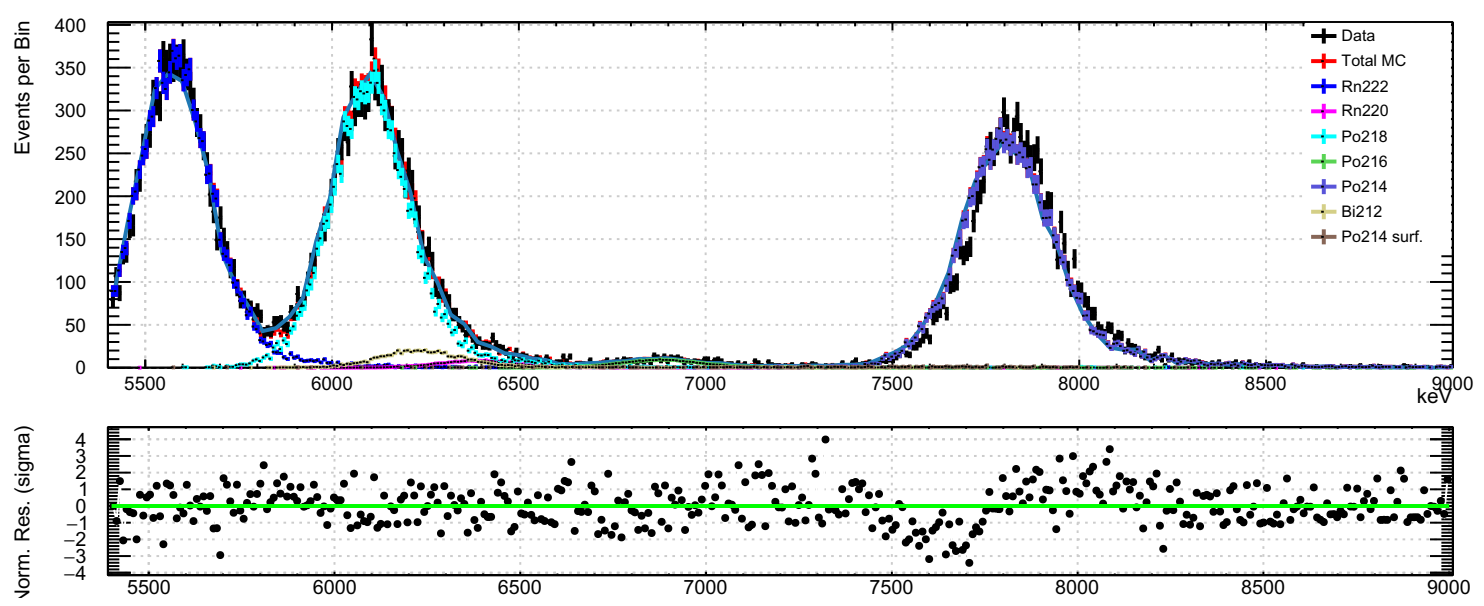

Figure B.2: Fit result when MC slopes in Figure 5.5 are shifted down by 1 sigma. See table B.3. $\chi^{2} / \mathrm{NDF}=1.53$

\begin{tabular}{|c|c|c|}
\hline Isotope & Activity (events $/ \mathbf{2 4 7 . 1 7}$ days) & Specific Activity $(\mu \mathrm{Bq} / \mathbf{k g})$ \\
\hline${ }^{222} \mathrm{Rn}$ & $10692.5_{-110.309}^{+110.946}$ & $0.152696_{-0.00473993}^{+0.00474296}$ \\
\hline${ }^{220} \mathrm{Rn}$ & $268.538_{-55.4111}^{+56.9814}$ & $0.00383491_{-0.000799234}^{+0.000821443}$ \\
\hline${ }^{218} \mathrm{Po}$ & $10565.2_{-145.95}^{+147.667}$ & $0.150878_{-0.00488431}^{+0.00489483}$ \\
\hline${ }^{216} \mathrm{Po}$ & $310.01_{-22.7274}^{+23.2192}$ & $0.00442715_{-0.000349487}^{+0.00035618}$ \\
\hline${ }^{214} \mathrm{Po}$ & $10768.5_{-112.835}^{+114.49}$ & $0.153781_{-0.00478195}^{+0.00478997}$ \\
\hline${ }^{212} \mathrm{Bi}$ & $2195.77_{-422.801}^{+429.374}$ & $0.0313571_{-0.00610728}^{+0.006201}$ \\
\hline${ }^{214} \mathrm{Po} \mathrm{surf.}$ & $115.068_{-32.9665}^{+37.4056}$ events in fit range \\
\hline
\end{tabular}

Table B.3: Fit result when MC slopes in Figure 5.5 are shifted down by 1 sigma. See Figure B.2. $\chi^{2} / \mathrm{NDF}=1.53$ 


\begin{tabular}{|c|c|c|}
\hline Isotope & $\begin{array}{c}\text { Delta Activity } \\
\text { (events } / \mathbf{2 4 7 . 1 7} \text { days })\end{array}$ & $\begin{array}{c}\text { Delta Specific } \\
\text { Activity }(\mu \mathrm{Bq} / \mathbf{k g})\end{array}$ \\
\hline${ }^{222} \mathrm{Rn}$ & -46.3413 & -0.000661786 \\
\hline${ }^{220} \mathrm{Rn}$ & -29.1479 & -0.000416252 \\
\hline${ }^{218} \mathrm{Po}$ & -588.504 & -0.00840425 \\
\hline${ }^{216} \mathrm{Po}$ & -1.2837 & $-1.83321 \mathrm{e}-05$ \\
\hline${ }^{214} \mathrm{Po}$ & 27.1622 & 0.000387895 \\
\hline${ }^{212} \mathrm{Bi}$ & 2181.77 & 0.0311572 \\
\hline${ }^{214} \mathrm{Po} \mathrm{surf}$. & -20.5777 events in fit range \\
\hline
\end{tabular}

Table B.4: Fit result when MC slopes in Figure 5.5 are shifted down by 1 sigma. See Figure B.2. $\chi^{2} / \mathrm{NDF}=1.53$ 

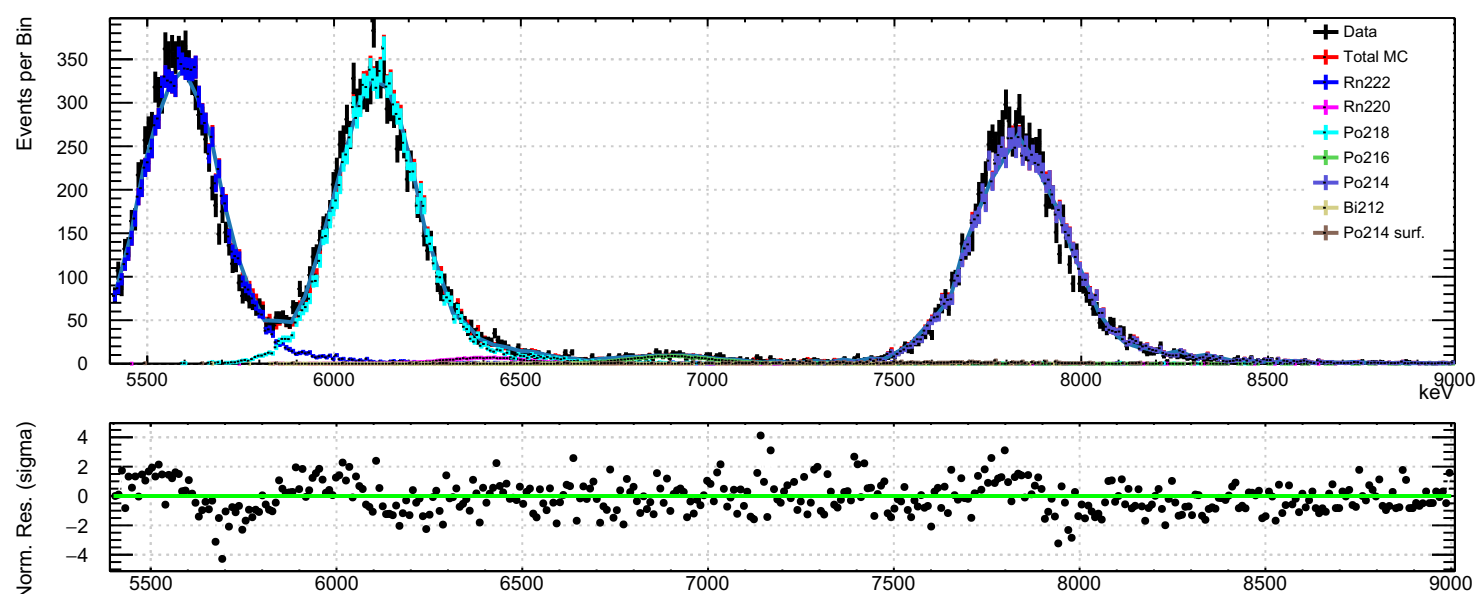

Figure B.3: Fit result when MC intercepts in Figure 5.6 are shifted up by 1 sigma. See table B.5. $\chi^{2} / \mathrm{NDF}=1.36$

\begin{tabular}{|c|c|c|}
\hline Isotope & Activity (events $/ \mathbf{2 4 7 . 1 7}$ days) & Specific Activity $(\mu \mathrm{Bq} / \mathbf{k g})$ \\
\hline${ }^{222} \mathrm{Rn}$ & $10741.4_{-110.831}^{+111.066}$ & $0.153394_{-0.0047617}^{+0.00476282}$ \\
\hline${ }^{220} \mathrm{Rn}$ & $237.191_{-58.5248}^{+38.0718}$ & $0.00338725_{-0.000841638}^{+0.000552662}$ \\
\hline${ }^{218} \mathrm{Po}$ & $11183.4_{-148.746}^{+117.581}$ & $0.159707_{-0.00513568}^{+0.00496814}$ \\
\hline${ }^{216} \mathrm{Po}$ & $307.845_{-23.1374}^{+23.1784}$ & $0.00439624_{-0.000354602}^{+0.000355148}$ \\
\hline${ }^{214} \mathrm{Po}$ & $10728.2_{-113.12}^{+114.46}$ & $0.153206_{-0.00476749}^{+0.00477401}$ \\
\hline${ }^{212} \mathrm{Bi}$ & $4.3885 \mathrm{e}-09_{-0}^{+62.3688}$ & $6.26708 \mathrm{e}-14_{-1.83483 e-15}^{+0.00089069}$ \\
\hline${ }^{214} \mathrm{Po} \mathrm{surf.}$ & $182.121_{-35.7082}^{+42.1499}$ events in fit range \\
\hline
\end{tabular}

Table B.5: Fit result when MC intercepts in Figure 5.6 are shifted up by 1 sigma. See Figure B.3. $\chi^{2} / \mathrm{NDF}=1.36$ 


\begin{tabular}{|c|c|c|}
\hline Isotope & $\begin{array}{c}\text { Delta Activity } \\
\text { (events } / \mathbf{2 4 7 . 1 7} \text { days })\end{array}$ & $\begin{array}{c}\text { Delta Specific } \\
\text { Activity }(\mu \mathbf{B q} / \mathbf{k g})\end{array}$ \\
\hline${ }^{222} \mathrm{Rn}$ & -0.426286 & $-6.08765 \mathrm{e}-06$ \\
\hline${ }^{220} \mathrm{Rn}$ & -61.8784 & -0.000883665 \\
\hline${ }^{218} \mathrm{Po}$ & 23.6435 & 0.000337646 \\
\hline${ }^{216} \mathrm{Po}$ & -6.01185 & $-8.58534 \mathrm{e}-05$ \\
\hline${ }^{214} \mathrm{Po}$ & -20.9827 & -0.000299647 \\
\hline${ }^{212} \mathrm{Bi}$ & 0.00024505 & $3.49949 \mathrm{e}-09$ \\
\hline${ }^{214} \mathrm{Po}$ surf. & \multicolumn{2}{|c|}{56.0668 events in fit range } \\
\hline
\end{tabular}

Table B.6: Fit result when MC intercepts in Figure 5.6 are shifted up by 1 sigma. See Figure B.3. $\chi^{2} / \mathrm{NDF}=1.36$ 

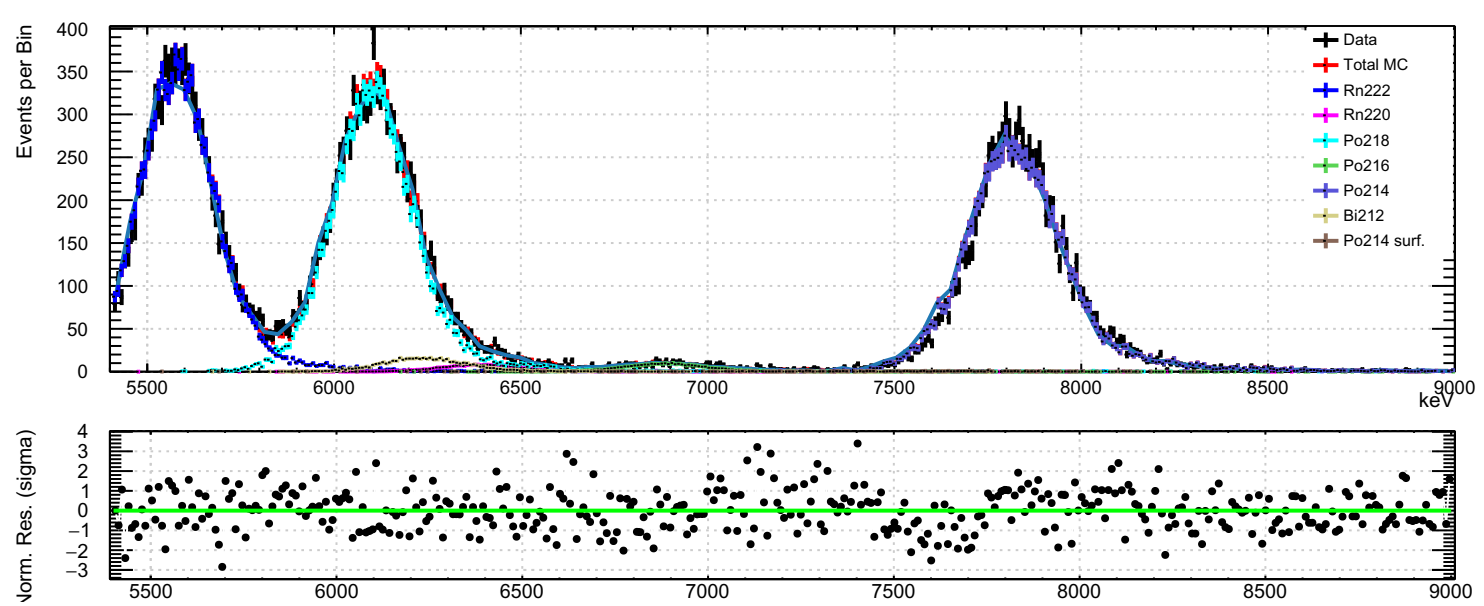

Figure B.4: Fit result when MC intercepts in Figure 5.6 are shifted down by 1 sigma. See table B.7. $\chi^{2} / \mathrm{NDF}=1.18$

\begin{tabular}{|c|c|c|}
\hline Isotope & Activity (events $/ \mathbf{2 4 7 . 1 7}$ days) & Specific Activity $(\mu \mathrm{Bq} / \mathbf{k g})$ \\
\hline${ }^{222} \mathrm{Rn}$ & $10699.1_{-110.585}^{+110.941}$ & $0.15279_{-0.00474385}^{+0.0047554}$ \\
\hline${ }^{220} \mathrm{Rn}$ & $272.585_{-56.9296}^{+56.7921}$ & $0.0038927_{-0.000820944}^{+0.000818998}$ \\
\hline${ }^{218} \mathrm{Po}$ & $10682.3_{-147.853}^{+147.181}$ & $0.15255_{-0.0049402}^{+0.00493611}$ \\
\hline${ }^{216} \mathrm{Po}$ & $323.921_{-22.6187}^{+23.1769}$ & $0.00462582_{-0.000350253}^{+0.000357618}$ \\
\hline${ }^{214} \mathrm{Po}$ & $10773.2_{-113.123}^{+113.449}$ & $0.153849_{-0.0047852}^{+0.00478678}$ \\
\hline${ }^{212} \mathrm{Bi}$ & $1767.19_{-428.893}^{+424.158}$ & $0.0252367_{-0.00616929}^{+0.0061017}$ \\
\hline${ }^{214} \mathrm{Po} \mathrm{surf.}$ & $70.6806_{-33.8916}^{+35.7925}$ events in fit range \\
\hline
\end{tabular}

Table B.7: Fit result when MC intercepts in Figure 5.6 are shifted down by 1 sigma. See Figure B.4. $\chi^{2} / \mathrm{NDF}=1.18$ 


\begin{tabular}{|c|c|c|}
\hline Isotope & $\begin{array}{c}\text { Delta Activity } \\
(\text { events } / \mathbf{2 4 7 . 1 7} \text { days })\end{array}$ & $\begin{array}{c}\text { Delta Specific } \\
\text { Activity }(\mu \mathrm{Bq} / \mathbf{k g})\end{array}$ \\
\hline${ }^{222} \mathrm{Rn}$ & -20.521 & -0.000293054 \\
\hline${ }^{220} \mathrm{Rn}$ & -21.5554 & -0.000307827 \\
\hline${ }^{218} \mathrm{Po}$ & -412.98 & -0.00589764 \\
\hline${ }^{216} \mathrm{Po}$ & -6.71831 & $-9.59421 \mathrm{e}-05$ \\
\hline${ }^{214} \mathrm{Po}$ & 16.5438 & 0.000236257 \\
\hline${ }^{212} \mathrm{Bi}$ & 1601.57 & 0.0228715 \\
\hline${ }^{214} \mathrm{Po} \mathrm{surf}$. & 8.51663 events in fit range \\
\hline
\end{tabular}

Table B.8: Fit result when MC intercepts in Figure 5.6 are shifted down by 1 sigma. See Figure B.4. $\chi^{2} / \mathrm{NDF}=1.18$ 

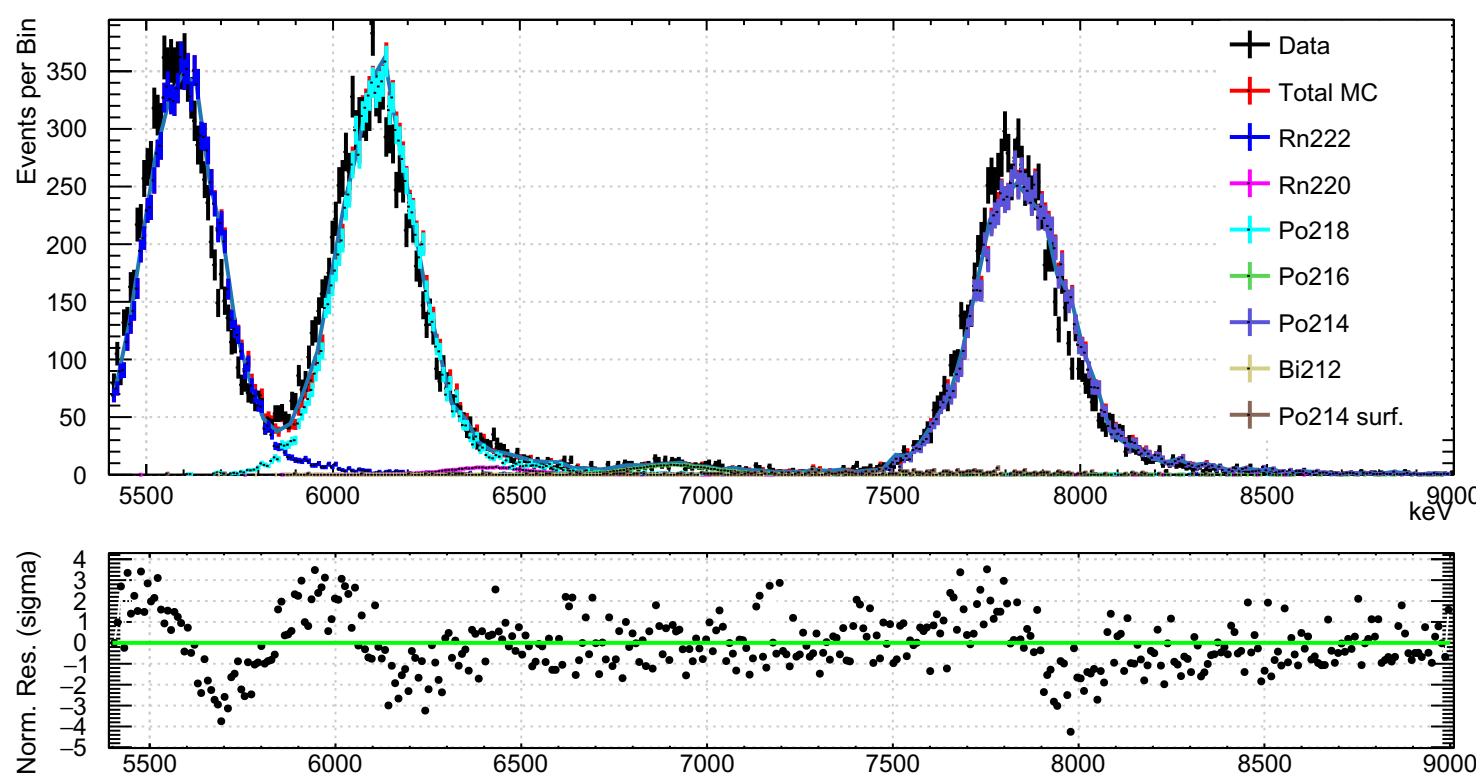

Figure B.5: Fit result when $\mathrm{MC}$ qPE is shifted up $0.2 \%$ after calibration. See table B.9. $\chi^{2} / \mathrm{NDF}=2.00$ 


\begin{tabular}{|c|c|c|}
\hline Isotope & Activity (events $/ \mathbf{2 4 7 . 1 7}$ days) & Specific Activity $(\mu \mathrm{Bq} / \mathbf{k g})$ \\
\hline${ }^{222} \mathrm{Rn}$ & $10659.9_{-109.605}^{+109.997}$ & $0.152231_{-0.00472375}^{+0.00072561}$ \\
\hline${ }^{220} \mathrm{Rn}$ & $214.69_{-56.483}^{+35.9015}$ & $0.00306592_{-0.000811595}^{+0.000520496}$ \\
\hline${ }^{218} \mathrm{Po}$ & $11091.9_{-143.863}^{+116.389}$ & $0.158399_{-0.00507219}^{+0.00492635}$ \\
\hline${ }^{216} \mathrm{Po}$ & $288.848_{-22.1508}^{+22.7623}$ & $0.00412495_{-0.000338599}^{+0.000346771}$ \\
\hline${ }^{214} \mathrm{Po}$ & $10602.5_{-113.168}^{+114.796}$ & $0.151411_{-0.00471831}^{+0.00472632}$ \\
\hline${ }^{212} \mathrm{Bi}$ & $1.10768 \mathrm{e}-05_{-0}^{+28.1252}$ & $1.58184 \mathrm{e}-10_{-4.63118 e-12}^{+0.000401647}$ \\
\hline${ }^{214} \mathrm{Po}$ surf. & $282.116_{-39.8157}^{+44.9278}$ events in fit range \\
\hline
\end{tabular}

Table B.9: Fit result when MC qPE is shifted up $0.2 \%$ after calibration. See Figure B.5. $\chi^{2} / \mathrm{NDF}=2.00$

\begin{tabular}{|c|c|c|}
\hline Isotope & $\begin{array}{c}\text { Delta Activity } \\
\text { (events/247.17 days) }\end{array}$ & $\begin{array}{c}\text { Delta Specific } \\
\text { Activity }(\mu \mathbf{B q} / \mathbf{k g})\end{array}$ \\
\hline${ }^{222} \mathrm{Rn}$ & -63.4779 & -0.000906509 \\
\hline${ }^{220} \mathrm{Rn}$ & -85.176 & -0.00121637 \\
\hline${ }^{218} \mathrm{Po}$ & -59.2465 & -0.00084608 \\
\hline${ }^{216} \mathrm{Po}$ & -23.9591 & -0.000342152 \\
\hline${ }^{214} \mathrm{Po}$ & -131.319 & -0.00187533 \\
\hline${ }^{212} \mathrm{Bi}$ & $-3.9855 \mathrm{e}-05$ & $-5.69157 \mathrm{e}-10$ \\
\hline${ }^{214} \mathrm{Po}$ surf. & \multicolumn{2}{|c|}{171.8 events in fit range } \\
\hline
\end{tabular}

Table B.10: Fit result when $\mathrm{MC}$ qPE is shifted up $0.2 \%$ after calibration. See Figure B.5. $\chi^{2} / \mathrm{NDF}=2.00$ 

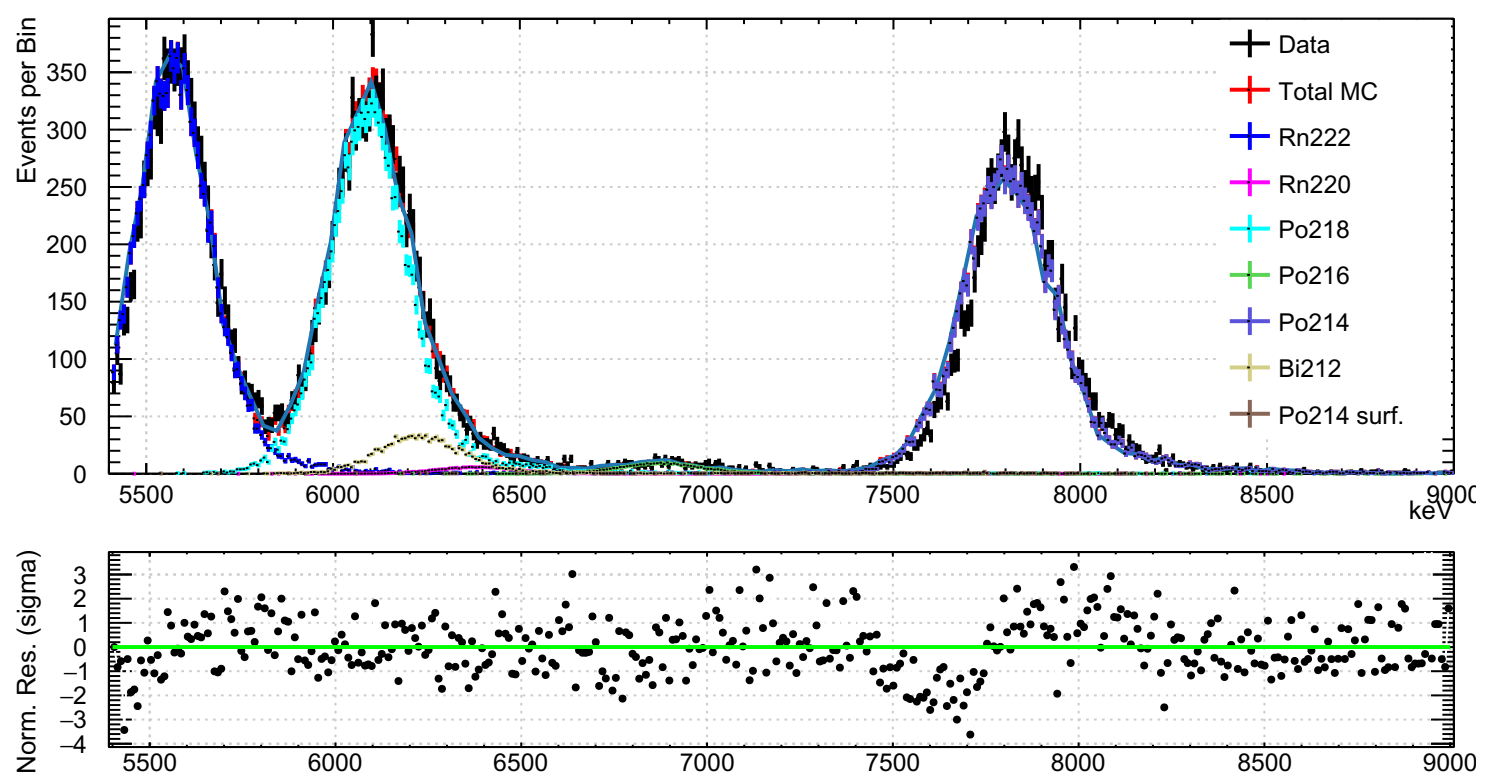

Figure B.6: Fit result when MC qPE is shifted down $0.2 \%$ after calibration. See table B.11. $\chi^{2} / \mathrm{NDF}=1.47$ 


\begin{tabular}{|c|c|c|}
\hline Isotope & Activity (events $/ \mathbf{2 4 7 . 1 7}$ days) & Specific Activity $(\mu \mathbf{B q} / \mathbf{k g})$ \\
\hline${ }^{222} \mathrm{Rn}$ & $10698_{-111.413}^{+111.311}$ & $0.152775_{-0.0047474}^{+0.00074691}$ \\
\hline${ }^{220} \mathrm{Rn}$ & $192.078_{-55.9428}^{+56.8839}$ & $0.00274301_{-0.000802928}^{+0.000816301}$ \\
\hline${ }^{218} \mathrm{Po}$ & $10207.1_{-145.632}^{+147.058}$ & $0.145765_{-0.00474738}^{+0.00475634}$ \\
\hline${ }^{216} \mathrm{Po}$ & $312.429_{-22.3992}^{+22.9473}$ & $0.00446169_{-0.000345519}^{+0.000352778}$ \\
\hline${ }^{214} \mathrm{Po}$ & $10744.2_{-112.701}^{+113.127}$ & $0.153434_{-0.00477174}^{+0.0047738}$ \\
\hline${ }^{212} \mathrm{Bi}$ & $3631.21_{-432.978}^{+439.031}$ & $0.0518562_{-0.00636688}^{+0.0064506}$ \\
\hline${ }^{214} \mathrm{Po}$ surf. & $70.7186_{-27.7025}^{+33.262}$ events in fit range \\
\hline
\end{tabular}

Table B.11: Fit result when MC qPE is shifted down $0.2 \%$ after calibration. See Figure B.6. $\chi^{2} / \mathrm{NDF}=1.47$

\begin{tabular}{|c|c|c|}
\hline Isotope & $\begin{array}{c}\text { Delta Activity } \\
\text { (events } / \mathbf{2 4 7 . 1 7} \text { days })\end{array}$ & $\begin{array}{c}\text { Delta Specific } \\
\text { Activity }(\mu \mathbf{B q} / \mathbf{k g})\end{array}$ \\
\hline${ }^{222} \mathrm{Rn}$ & -25.3238 & -0.000361641 \\
\hline${ }^{220} \mathrm{Rn}$ & -107.788 & -0.00153929 \\
\hline${ }^{218} \mathrm{Po}$ & -943.966 & -0.0134805 \\
\hline${ }^{216} \mathrm{Po}$ & -0.378836 & $-5.41003 \mathrm{e}-06$ \\
\hline${ }^{214} \mathrm{Po}$ & 10.3299 & 0.000147518 \\
\hline${ }^{212} \mathrm{Bi}$ & 3631.21 & 0.0518562 \\
\hline${ }^{214} \mathrm{Po}$ surf. & -39.5975 events in fit range \\
\hline
\end{tabular}

Table B.12: Fit result when $\mathrm{MC}$ qPE is shifted down $0.2 \%$ after calibration. See Figure B.6. $\chi^{2} / \mathrm{NDF}=1.47$ 

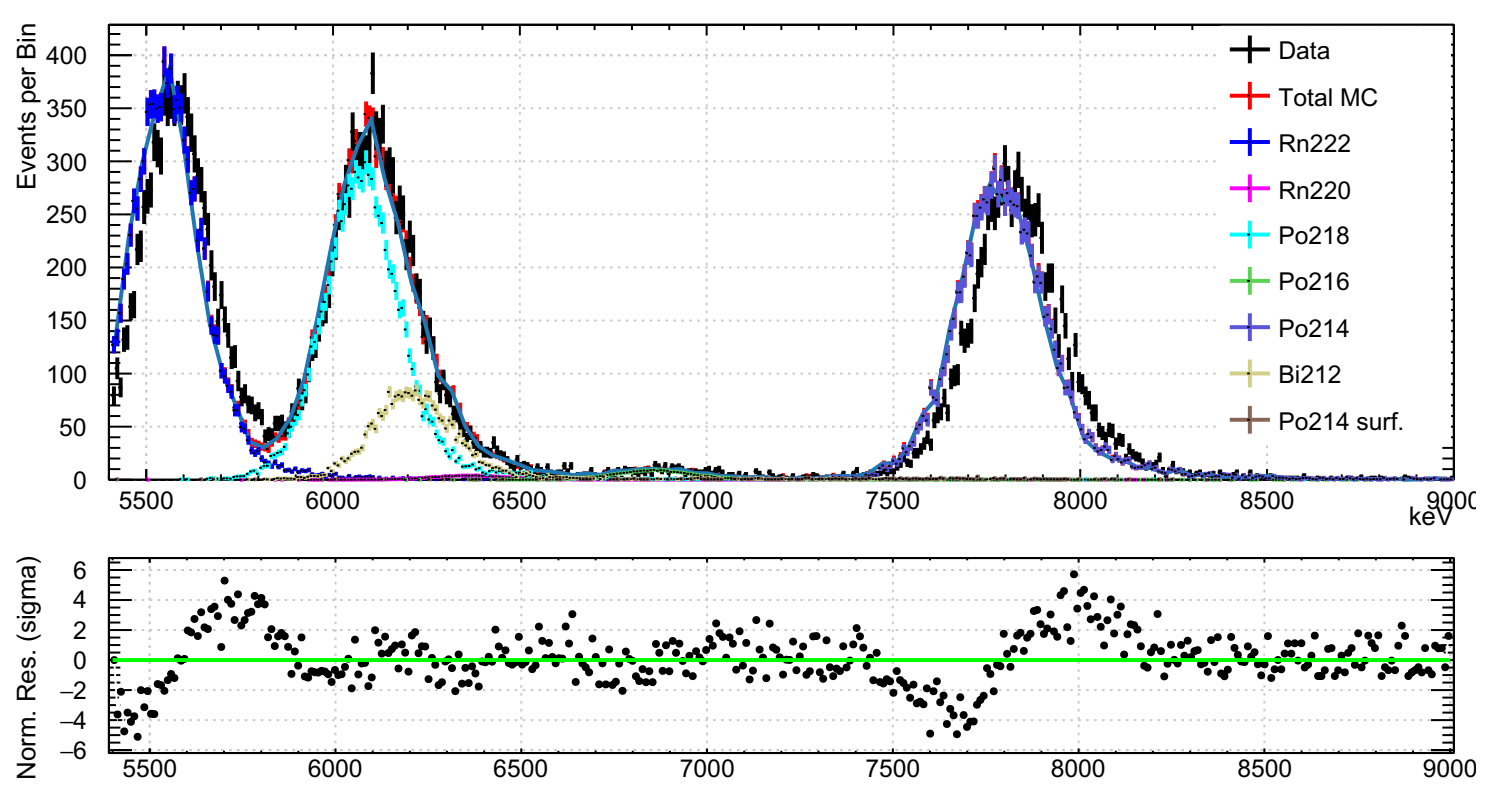

Figure B.7: Fit result when MC fprompt is shifted up $0.2 \%$ after calibration. See table B.13. $\chi^{2} / \mathrm{NDF}=3.49$ 


\begin{tabular}{|c|c|c|}
\hline Isotope & Activity (events $/ \mathbf{2 4 7 . 1 7}$ days) & Specific Activity $(\mu \mathrm{Bq} / \mathbf{k g})$ \\
\hline${ }^{222} \mathrm{Rn}$ & $10692.4_{-111.397}^{+111.72}$ & $0.152695_{-0.0047451}^{+0.00474665}$ \\
\hline${ }^{220} \mathrm{Rn}$ & $124.31_{-53.8537}^{+54.8155}$ & $0.00177524_{-0.000770822}^{+0.000784526}$ \\
\hline${ }^{218} \mathrm{Po}$ & $8821.25_{-139.694}^{+140.606}$ & $0.125974_{-0.00419312}^{+0.00419933}$ \\
\hline${ }^{216} \mathrm{Po}$ & $286.421_{-22.1354}^{+22.6174}$ & $0.00409029_{-0.000338031}^{+0.00034447}$ \\
\hline${ }^{214} \mathrm{Po}$ & $10716.3_{-112.541}^{+113.112}$ & $0.153036_{-0.00475999}^{+0.00476275}$ \\
\hline${ }^{212} \mathrm{Bi}$ & $8637.4_{-438.239}^{+440.57}$ & $0.123348_{-0.00722554}^{+0.00725439}$ \\
\hline${ }^{214} \mathrm{Po}$ surf. & $109.053_{-33.5513}^{+33.3517}$ events in fit range \\
\hline
\end{tabular}

Table B.13: Fit result when MC fprompt is shifted up $0.2 \%$ after calibration. See Figure B.7. $\chi^{2} / \mathrm{NDF}=3.49$

\begin{tabular}{|c|c|c|}
\hline Isotope & $\begin{array}{c}\text { Delta Activity } \\
\text { (events/247.17 days) }\end{array}$ & $\begin{array}{c}\text { Delta Specific } \\
\text { Activity }(\mu \mathbf{B q} / \mathbf{k g})\end{array}$ \\
\hline${ }^{222} \mathrm{Rn}$ & -30.9474 & -0.00044195 \\
\hline${ }^{220} \mathrm{Rn}$ & -175.556 & -0.00250706 \\
\hline${ }^{218} \mathrm{Po}$ & -2329.85 & -0.0332719 \\
\hline${ }^{216} \mathrm{Po}$ & -26.386 & -0.000376811 \\
\hline${ }^{214} \mathrm{Po}$ & -17.5514 & -0.000250647 \\
\hline${ }^{212} \mathrm{Bi}$ & 8637.4 & 0.123348 \\
\hline${ }^{214} \mathrm{Po}$ surf. & \multicolumn{2}{|c|}{-1.26301 events in fit range } \\
\hline
\end{tabular}

Table B.14: Fit result when MC fprompt is shifted up $0.2 \%$ after calibration. See Figure B.7. $\chi^{2} / \mathrm{NDF}=3.49$ 

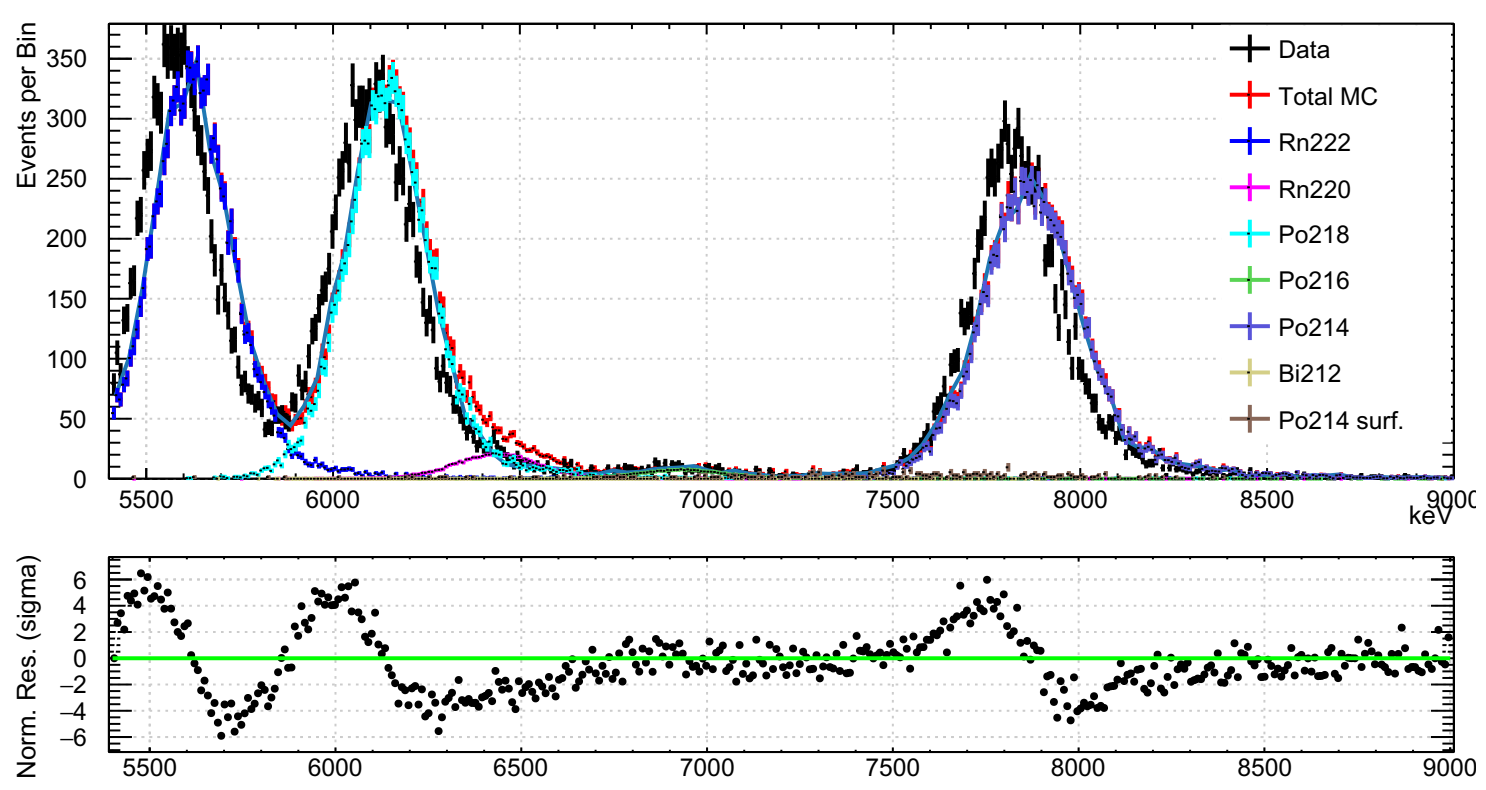

Figure B.8: Fit result when MC fprompt is shifted down $0.2 \%$ after calibration. See table B.15. $\chi^{2} / \mathrm{NDF}=6.23$ 


\begin{tabular}{|c|c|c|}
\hline Isotope & Activity (events $/ \mathbf{2 4 7 . 1 7}$ days) & Specific Activity $(\mu \mathrm{Bq} / \mathbf{k g})$ \\
\hline${ }^{222} \mathrm{Rn}$ & $10791.5_{-109.97}^{+110.535}$ & $0.15411_{-0.00477741}^{+0.0047807}$ \\
\hline${ }^{220} \mathrm{Rn}$ & $719.346_{-61.984}^{+30.5806}$ & $0.0102728_{-0.000934873}^{+0.000530257}$ \\
\hline${ }^{218} \mathrm{Po}$ & $11104.5_{-146.521}^{+116.598}$ & $0.158581_{-0.00509253}^{+0.00493236}$ \\
\hline${ }^{216} \mathrm{Po}$ & $269.971_{-21.6245}^{+22.3787}$ & $0.00385536_{-0.000328795}^{+0.00033831}$ \\
\hline${ }^{214} \mathrm{Po}$ & $10519.6_{-114.883}^{+116.473}$ & $0.150228_{-0.00469427}^{+0.00470225}$ \\
\hline${ }^{212} \mathrm{Bi}$ & $1.38042 \mathrm{e}-07_{-0}^{+12.3945}$ & $1.97134 \mathrm{e}-12_{-5.77152 e-14}^{+0.000177002}$ \\
\hline${ }^{214} \mathrm{Po}$ surf. & $398.39_{-48.9853}^{+58.1737}$ events in fit range \\
\hline
\end{tabular}

Table B.15: Fit result when MC fprompt is shifted down $0.2 \%$ after calibration. See Figure B.8. $\chi^{2} / \mathrm{NDF}=6.23$

\begin{tabular}{|c|c|c|}
\hline Isotope & $\begin{array}{c}\text { Delta Activity } \\
\text { (events } / \mathbf{2 4 7 . 1 7} \text { days })\end{array}$ & $\begin{array}{c}\text { Delta Specific } \\
\text { Activity }(\mu \mathbf{B q} / \mathbf{k g})\end{array}$ \\
\hline${ }^{222} \mathrm{Rn}$ & 68.1204 & 0.000972806 \\
\hline${ }^{220} \mathrm{Rn}$ & 419.48 & 0.00599046 \\
\hline${ }^{218} \mathrm{Po}$ & -46.5551 & -0.000664839 \\
\hline${ }^{216} \mathrm{Po}$ & -42.8368 & -0.000611739 \\
\hline${ }^{214} \mathrm{Po}$ & -214.197 & -0.00305888 \\
\hline${ }^{212} \mathrm{Bi}$ & $-5.07937 \mathrm{e}-05$ & $-7.25369 \mathrm{e}-10$ \\
\hline${ }^{214} \mathrm{Po}$ surf. & \multicolumn{2}{|c|}{288.074 events in fit range } \\
\hline
\end{tabular}

Table B.16: Fit result when MC fprompt is shifted down $0.2 \%$ after calibration. See Figure B.8. $\chi^{2} / \mathrm{NDF}=6.23$ 

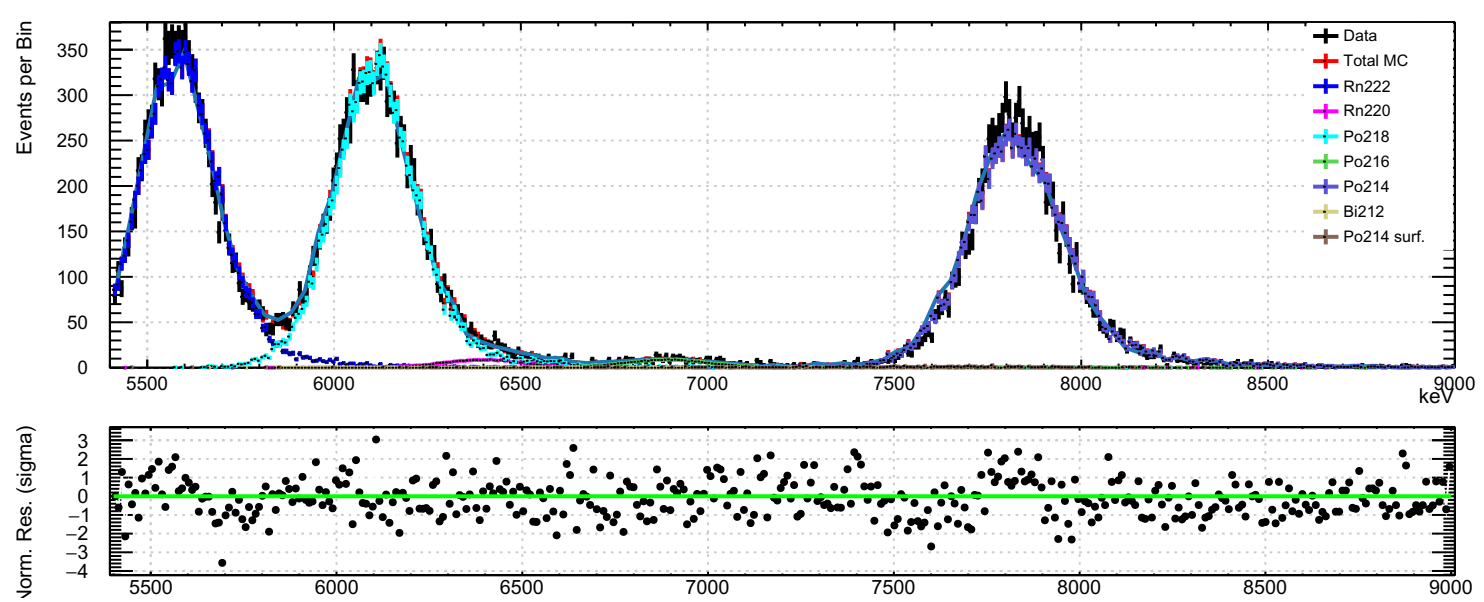

Figure B.9: Fit result when resolution term is shifted up by 1 sigma and normalizations are refitted. See Figure B.17. $\chi^{2} / \mathrm{NDF}=1.11$

\begin{tabular}{|c|c|c|}
\hline Isotope & Activity (events $/ \mathbf{2 4 7 . 1 7}$ days) & Specific Activity $(\mu \mathrm{Bq} / \mathbf{k g})$ \\
\hline${ }^{222} \mathrm{Rn}$ & $10744.9_{-110.949}^{+111.379}$ & $0.153445_{-0.00476365}^{+0.00476569}$ \\
\hline${ }^{220} \mathrm{Rn}$ & $302.821_{-59.8671}^{+39.9674}$ & $0.0043245_{-0.000864267}^{+0.000584635}$ \\
\hline${ }^{218} \mathrm{Po}$ & $11157.6_{-148.606}^{+117.51}$ & $0.159338_{-0.00512499}^{+0.00495761}$ \\
\hline${ }^{216} \mathrm{Po}$ & $313.805_{-23.0576}^{+23.1817}$ & $0.00448135_{-0.000354455}^{+0.000356102}$ \\
\hline${ }^{214} \mathrm{Po}$ & $10749.4_{-113.228}^{+113.584}$ & $0.153509_{-0.00477634}^{+0.0047786}$ \\
\hline${ }^{212} \mathrm{Bi}$ & $0.00084448_{-0}^{+311.504}$ & $1.20598 \mathrm{e}-08_{-3.53076 e-10}^{+0.00444849}$ \\
\hline${ }^{214} \mathrm{Po} \mathrm{surf.}$ & $131.408_{-36.692}^{+37.777}$ events in fit range \\
\hline
\end{tabular}

Table B.17: Fit result when resolution term is shifted up by 1 sigma and normalizations are refitted. See Figure B.9. $\chi^{2} / \mathrm{NDF}=1.11$ 


\begin{tabular}{|c|c|c|}
\hline Isotope & $\begin{array}{c}\text { Delta Activity } \\
\text { (events } / \mathbf{2 4 7 . 1 7} \text { days })\end{array}$ & $\begin{array}{c}\text { Delta Specific } \\
\text { Activity }(\mu \mathbf{B q} / \mathbf{k g})\end{array}$ \\
\hline${ }^{222} \mathrm{Rn}$ & 3.48059 & $4.97052 \mathrm{e}-05$ \\
\hline${ }^{220} \mathrm{Rn}$ & 17.2544 & 0.000246404 \\
\hline${ }^{218} \mathrm{Po}$ & 24.6254 & 0.000351667 \\
\hline${ }^{216} \mathrm{Po}$ & 0.430546 & $6.14849 \mathrm{e}-06$ \\
\hline${ }^{214} \mathrm{Po}$ & 5.3423 & $7.62917 \mathrm{e}-05$ \\
\hline${ }^{212} \mathrm{Bi}$ & 4.67096 & $6.67045 \mathrm{e}-05$ \\
\hline${ }^{214} \mathrm{Po} \mathrm{surf.}$ & -2.31087 events in fit range \\
\hline
\end{tabular}

Table B.18: Fit result when resolution term is shifted up by 1 sigma and normalizations are refitted. See Figure B.9. $\chi^{2} / \mathrm{NDF}=1.11$ 

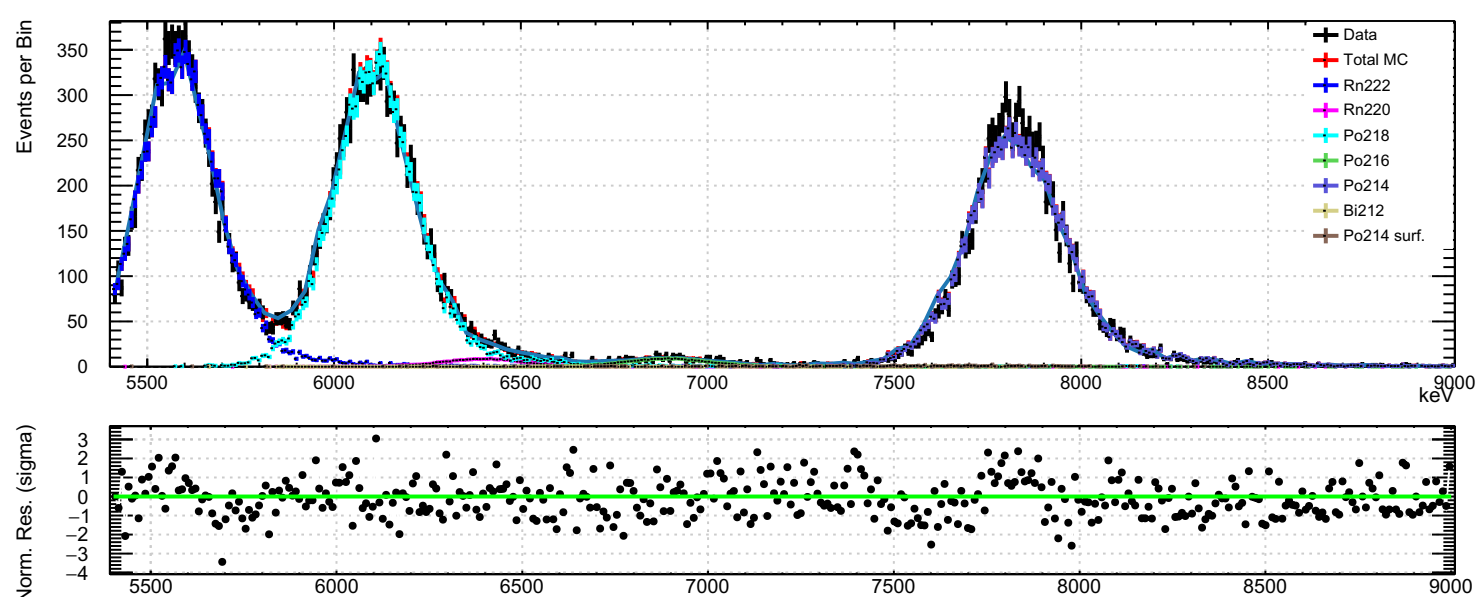

Figure B.10: Fit result when resolution term is shifted down by 1 sigma and normalizations are refitted. See Figure B.19. $\chi^{2} / \mathrm{NDF}=1.10$

\begin{tabular}{|c|c|c|}
\hline Isotope & Activity (events $/ \mathbf{2 4 7 . 1 7}$ days) & Specific Activity $(\mu \mathrm{Bq} / \mathbf{k g})$ \\
\hline${ }^{222} \mathrm{Rn}$ & $10744.2_{-110.967}^{+111.266}$ & $0.153435_{-0.00476346}^{+0.00476488}$ \\
\hline${ }^{220} \mathrm{Rn}$ & $299.779_{-59.7482}^{+40.0075}$ & $0.00428105_{-0.000862402}^{+0.000584921}$ \\
\hline${ }^{218} \mathrm{Po}$ & $11162_{-150.259}^{+117.608}$ & $0.159401_{-0.0051365}^{+0.0049598}$ \\
\hline${ }^{216} \mathrm{Po}$ & $316.725_{-22.5904}^{+22.9967}$ & $0.00452305_{-0.000348727}^{+0.000354101}$ \\
\hline${ }^{214} \mathrm{Po}$ & $10755.5_{-113.074}^{+113.489}$ & $0.153596_{-0.004778}^{+0.00478}$ \\
\hline${ }^{212} \mathrm{Bi}$ & $0.00258427_{-0}^{+372.717}$ & $3.69052 \mathrm{e}-08_{-1.08048 e-09}^{+0.00532266}$ \\
\hline${ }^{214} \mathrm{Po} \mathrm{surf.}$ & $118.864_{-34.5578}^{+35.643}$ events in fit range \\
\hline
\end{tabular}

Table B.19: Fit result when resolution term is shifted down by 1 sigma and normalizations are refitted. See Figure B.10. $\chi^{2} / \mathrm{NDF}=1.10$ 


\begin{tabular}{|c|c|c|}
\hline Isotope & $\begin{array}{c}\text { Delta Activity } \\
\text { (events } / \mathbf{2 4 7 . 1 7} \text { days })\end{array}$ & $\begin{array}{c}\text { Delta Specific } \\
\text { Activity }(\mu \mathbf{B q} / \mathbf{k g})\end{array}$ \\
\hline${ }^{222} \mathrm{Rn}$ & 1.72565 & $2.46434 \mathrm{e}-05$ \\
\hline${ }^{220} \mathrm{Rn}$ & 0.797347 & $1.13867 \mathrm{e}-05$ \\
\hline${ }^{218} \mathrm{Po}$ & 1.33857 & $1.91157 \mathrm{e}-05$ \\
\hline${ }^{216} \mathrm{Po}$ & 2.90462 & $4.14799 \mathrm{e}-05$ \\
\hline${ }^{214} \mathrm{Po}$ & 7.69204 & 0.000109848 \\
\hline${ }^{212} \mathrm{Bi}$ & 0.000881666 & $1.25908 \mathrm{e}-08$ \\
\hline${ }^{214} \mathrm{Po} \mathrm{surf.}$ & -9.6743 events in fit range \\
\hline
\end{tabular}

Table B.20: Fit result when resolution term is shifted down by 1 sigma and normalizations are refitted. See Figure B.10. $\chi^{2} / \mathrm{NDF}=1.10$ 


\section{Appendix C}

\section{Correlation Matrices for 5.5 MeV Solar Axion Search}

The correlation matrices from the final AmBe calibration and final blinded physics fits are shown below. Due to the large number of parameters, the text is difficult to read without the use of a pdf viewer. 


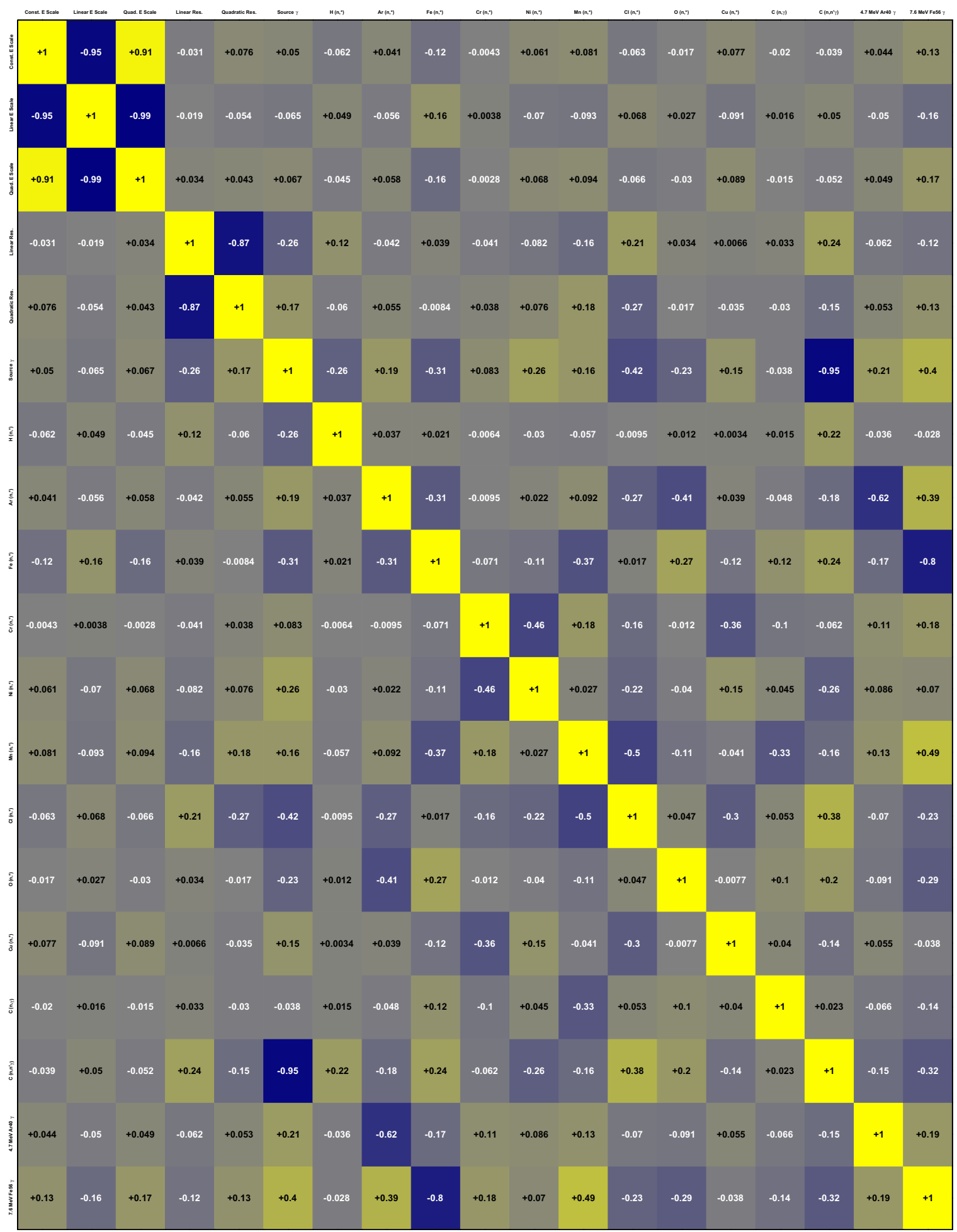

Figure C.1: Correlation matrix of parameters that were free to vary in the full AmBe calibration fit. 


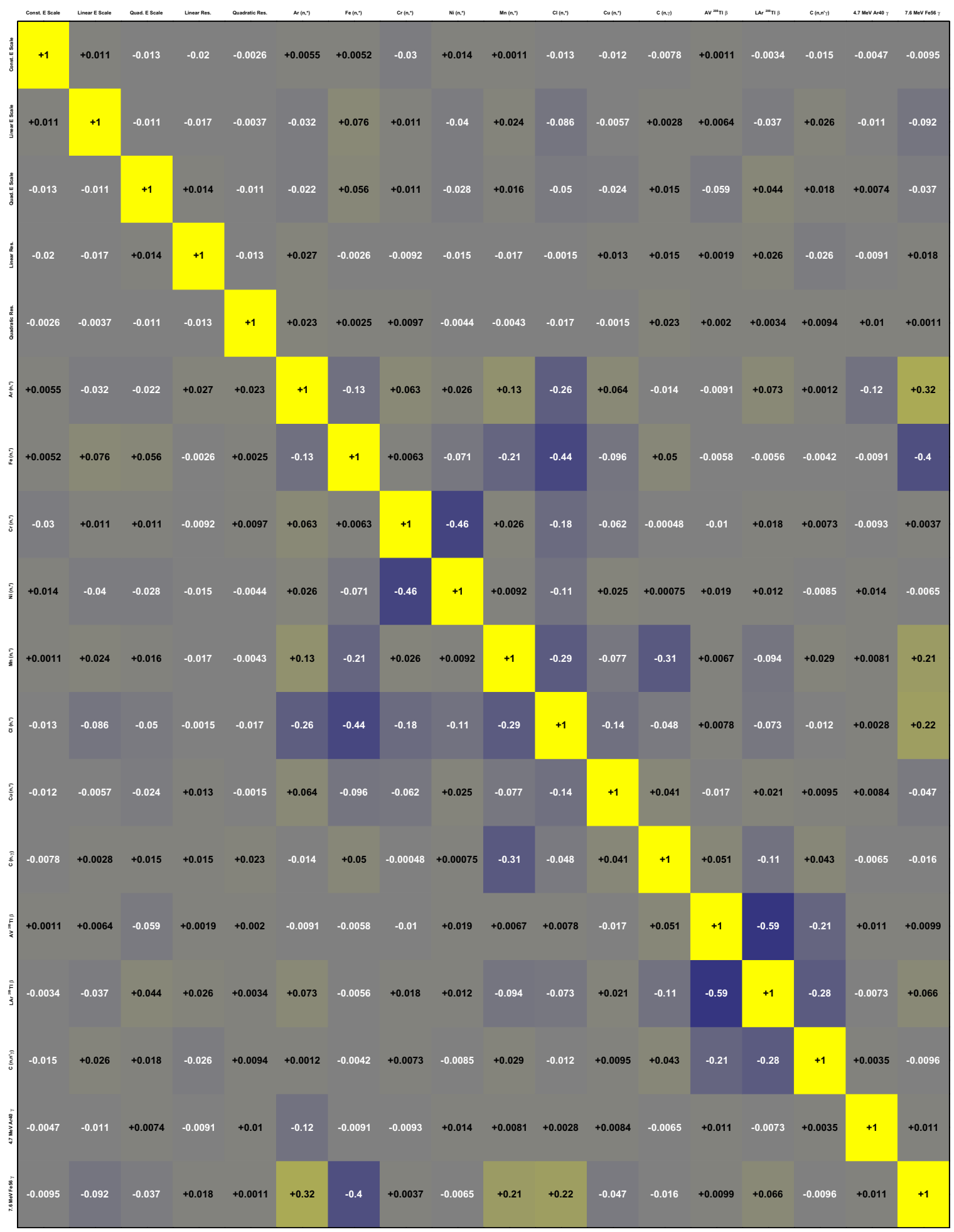

Figure C.2: Correlation matrix of parameters that were free to vary in the blinded physics fit. 